\title{
Faunal Biogeography, Community Structure, and Genetic Connectivity of North Atlantic Seamounts
}

\author{
By \\ Walter W. Cho \\ A.B., Harvard University, 2000 \\ Submitted in partial fulfillment of the requirements for the degree of \\ Doctor of Philosophy \\ at the \\ MASSACHUSETTS INSTITUTE OF TECHNOLOGY \\ and the \\ WOODS HOLE OCEANOGRAPHIC INSTITUTION
}

September 2008

(C) 2008 Walter W. Cho, All rights reserved.

The author hereby grants to MIT and WHOI permission to reproduce and to distribute publicly paper and electronic copies of this thesis document in whole or in part in any medium now known or hereafter created.

Signature of Author

Joint Program in Biological Oceanography Massachusetts Institute of Technology and Woods Hole Oceanographic Institution September 2, 2008

Certified by

Timothy M. Shank

Thesis Supervisor

Accepted by

Edward F. DeLong

Chair, Joint Committee for Biological Oceanography

Woods Hole Oceanographic Institution 


\title{
Faunal Biogeography, Community Structure, and Genetic Connectivity of North Atlantic Seamounts
}

\author{
by \\ Walter W. Cho \\ Submitted to the Department of Biology on September 2, 2008 \\ in Partial Fulfillment of the Requirements for the Degree of \\ Doctor of Philosophy in Biological Oceanography
}

\begin{abstract}
The mechanisms of faunal dispersal across ocean basins are key unknowns toward understanding of the modern biogeography and biodiversity of deep-sea fauna. Seamounts are considered to play a defining role in faunal evolution, acting as regional centers of speciation, "stepping-stones" for dispersal, and/or refugia for deep-sea populations. The overarching goal of this dissertation was to examine the role of seamounts in structuring marine biodiversity and biogeography. This study focused on North Atlantic seamounts, specifically the New England seamount chain, the Corner Rise seamounts, and Muir seamount, areas damaged and threatened by deep-sea fisheries and currently a focus of conservation efforts. Videographic analyses of biological community structure revealed distinct faunal assemblages, dominated by the Porifera, Cnidaria, and Echinodermata and structured by geographic region, depth regions (with apparent taxonomic breaks at $1300 \mathrm{~m}, 2300 \mathrm{~m}$, and $2600 \mathrm{~m}$ ), and substrate type (including natural/anthropogenic and abiotic substrates and biotic substrates). Amongst these assemblages, seven highly specific coral host- invertebrate associate relationships were identified. To investigate whether or not these broad community patterns were discernible at a genetic level, the 16S mtDNA gene was utilized as a genetic "barcode" within the Class Ophiuroidea, through which 22 putative species were identified, including four target species (Asteroschema clavigera, Ophiocreas oedipus, Ophioplinthaca abyssalis, and Ophioplinthaca chelys) for subsequent population genetic studies. Analyses of mitochondrial 16S and COI gene sequences revealed evidence for recent population expansion and estimates of recent high gene flow across all four species throughout the North Atlantic seamount region. However, genetic differentiation within populations of $A$. clavigera and $O$. chelys within seamount regions was significant, suggesting that historical diversification has been mediated by a long-distance dispersal mechanism that homogenizes this genetic signal on a regional scale. In addition, comparisons of all ophiuroid populations revealed no congruent pattern of historical migration amongst seamounts, which may also be attributed to the varying levels of host specificity and reproductive strategy of each ophiuroid species. These results will guide future studies and conservation efforts to protect seamount communities vulnerable to deep-sea fishery activities.
\end{abstract}

Thesis Supervisor: Timothy M. Shank Title: Associate Scientist 


\section{Table of Contents}

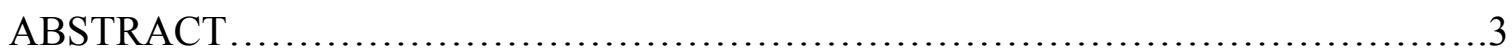

ACKNOWLEDGEMENTS ...................................................

CHAPTER 1:

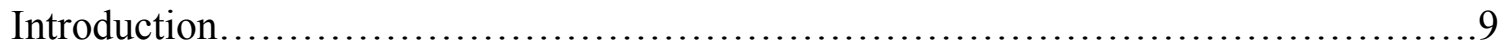

CHAPTER 2:

Fauna-habitat associations and community structure on the

New England and Corner Rise Seamounts

CHAPTER 3:

Identifying ophiuroids from the North Atlantic seamounts

using DNA barcodes.

CHAPTER 4:

Patterns of gene flow of four species of ophiuroids

from North Atlantic seamounts...

CHAPTER 5:

Conclusions. 


\section{Acknowledgements}

I have many people to thank, for this dissertation is not the result of just my own work. I have been helped a lot along the way. I want to first thank my wife Carol who had to put up with a lot and has suffered much but all the way helped me in every way she could. I love you and I could not have done this without you. I also want to thank my family, my parents and sister for always supporting me no matter what I wanted to do or pursue.

I want to sincerely thank my advisor, Tim Shank. Thank you for giving me the amazing opportunity to work on this project, but more than that for being my mentor in science, patiently guiding me and giving me the independence to pursue this research and grow as a scientist. I want to thank my committee members who have all been a great help and guide to me throughout my education, as well as patiently bearing with all of my struggles to schedule committee meetings. I would like to thank Glenn Flierl who has always given me keen insight into my research and helped me to focus in on the important aspects of my research and Dennis McGillicuddy for giving me great advice and direction in my research as well as guiding me to resources to help me understand the current systems in the North Atlantic. I would like to thank Jesús Pineda for always giving me great input on my research and reminding me to explain and define the fundamental principles I take for granted and Les Watling for his great knowledge of the seamount invertebrate fauna as well as always challenging me to ensure the quality of my research.

I would like to gratefully acknowledge the submersible pilots and crews of the $R / V$ Ron Brown/Hercules at the Institute for Exploration, and $R / V$ Atlantis/Alvin for their invaluable assistance. I also want to deeply thank the principal investigators of the DeepAtlantic Stepping Stones research cruise from which I got the majority of my samples: Dr. Jess Adkins (USGS), Dr. Peter Auster (Univ. of Connecticut), Dr. Scott France (Univ. of Louisiana), Dr. Lauren Mullineaux (WHOI), Dr. Rhian Waller (Univ. of Hawaii), and Dr. Les Watling (Univ. of Hawaii). I also want thank Dr. Rhian Waller (Univ. of Hawaii) for providing me samples from Picket seamount, Dr. John Moore (Florida Atlantic University) for samples from Bear seamount, and Dr. Rebecca Hunter and Dr. Ken Halanych (Auburn University) for providing various Ophiurida samples.

I would like to thank those who assisted in morpho-species determination, including S. France (Univ. of Louisiana), L. Watling and R. Waller (University of Hawaii), J. Adkins (California Institute of Technology) and S. Cairns (Smithsonian Institution) for the Cnidaria; C. Ahearn (Smithsonian Institution) for the Echinodermata; M. Nizinski (Smithsonian Institution) and Kareen Schnabel (NIWA) for the Arthropoda; and P. Auster (University of Connecticut) and J. Moore (Florida Atlantic University) for the Chordata. Thanks also to William Lange and the Advanced Imaging and Visualization Laboratory at the Woods Hole Oceanographic Institution, who generously provided technical advice and image analysis support, and D. Scheirer (USGS) and A. Gontz (University of Massachusetts - Boston) for multibeam mapping support. 
I would like to thank Dr. Dave Pawson and Cynthia Ahearn (Smithsonian Institution) for all of their help in learning the morphological characters of ophiuroids and Dr. Tim O'Hara and Dr. Paula Cisternas (Museum Victoria) for invaluable help with molecular techniques with ophiuroids and primers.

I would like to thank all those in the Shank lab who have helped me immensely in learning what I was doing and also making it fun, including Diane Adams, Amy BacoTaylor, Stace Beaulieu, Kate Buckman, Abby Fusaro, Breea Govenar, Taylor Heyl Muric, Rhian Waller, and all the guest students in lab.

Finally I would like to thank Berkland Baptist Church, Pastor Paul and Becky JDSN. Without the prayers, love, and support of all my friends at church I never would have survived. I would like to thank the members of Harvard ABSK, Angela SMN and the other staff and students for their prayers and love. I would like to thank Patrick, Mike, Tim, and Vallent for putting up with me during the past six years and always being there to support me and help me relax and recover. I would like to thank Dan hyung for his amazing love and support for me, helping me survive $\mathrm{my} \mathrm{PhD}$ and life in general. I would like to finally thank God for creating such a beautiful and interesting world that I could study.

Financial support was provided by the Ocean Life Institute, Deep-Ocean Exploration Institute, and Academic Programs Office of the Woods Hole Oceanographic Institution, the Census of Marine Life for Seamounts (CenSeam) through their mini-grant program (Grant \#12301), the Office of Ocean Exploration, National Oceanic and Atmospheric Administration (NA05OAR4601054) and the National Science Foundation (OCE-0624627; OCE-0451983; OCE-0647612). 


\section{Chapter 1: Introduction}

Seamounts are common geological features of the ocean floor. Although known since the $19^{\text {th }}$ century, many questions still remain about the biogeographic features of these distinctive deep-sea habitats, such as what types of biological communities they support, what factors are important for creating, structuring, and maintaining those communities, and what role they play in marine biodiversity and biogeography (Brewin et al. 2007).

\section{Seamount Geology and Oceanography}

There have been estimates of more than 800 seamounts in the Atlantic, 30,00050,000 seamounts in the Pacific, and 100,000 seamounts found in all the world oceans (Epp and Smoot 1989; Rogers 1994; Rogers 2004). They have been defined as underwater mountains that rise at least $1000 \mathrm{~m}$ off of the seafloor(Menard 1964). This definition is artificial, as the biological, ecological, and hydrographic characteristics of seamounts are not dramatically different when their summits are below this height. Alternatively, they have been defined as mountains that rise from the seafloor but do not come above the sea surface (Epp and Smoot 1989). They are often formed due to on-axis and off-axis ridge volcanism and more rarely by tectonic shifts (Batiza 2001; Epp and Smoot 1989; Fryer and Fryer 1987). Seamounts formed on-axis are generally small and form near medium and fast-spreading ridges, most likely due to mantle upwelling and melting in a large area beneath the ridge system. Seamounts formed by offaxis volcanism can be formed by mantle plumes, resulting in large oceanic plateaus and linear volcanic chains or by off-axis volcanism resulting from intraplate mantle upwelling or athenosphere melt rising due to intraplate stresses (Batiza 2001; Epp and Smoot 1989). 
They can also have a variety of forms but are generally conical in shape with a circular, elliptical or more elongate base (Rogers 1994).

Seamounts have an observable impact on ocean circulation, which can vary widely depending upon the morphology of the seamount. The interactions of seamounts and ocean currents have been seen at a variety of spatial scales. On the scale of ocean basins, the Emperor seamount chain has been shown to deflect the Kuroshio Extension and subarctic currents (Roden and Taft 1985; Roden et al. 1982; Rogers 1994; Vastano et al. 1985). The New England Seamount chain has also been shown to interact with the Gulf Stream, which bisects the chain (Ezer 1994; Qiu 1994).

At smaller spatial scales, seamounts have been shown to form trapped waves, reflect, amplify, and distort internal waves, and amplify diurnal and semidiurnal tides (Rogers 1994 and references therein). One of the most interesting effects seamounts have on the hydrographic regime in this scale is the formation of Taylor columns, regions with closed streamlines that form as ocean currents pass and interact with seamounts. These result in closed eddies and localized upwelling (Hogg 1973; Mullineaux and Mills 1997; Rogers 1994; Taylor 1917). Taylor columns have been observed on a variety of seamounts of varying sizes in both the Pacific and Atlantic Oceans and also have been shown to be able to last for significant amounts of time (Rogers 1994). One eddy was tracked over the Corner Rise seamounts for 6 weeks, although this may be a gyre resulting from the interaction of the Gulf Stream with the New England Seamount chain rather than a Taylor Column formation (Qiu 1994; Richardson 1980). These closed recirculation cells have been suggested as a mechanism for larval retention and also increased productivity, both of which lead to the interesting biological characteristics of seamounts.

\section{Seamount Biodiversity}

Due to their volcanic history, most seamounts provide a unique hard substrate within the deep-sea benthos, which is otherwise dominated by the sandy abyssal plains. 
These hard substrates serve as important biological habitats within the deep-sea. This is seen in the large faunal assemblages that they support, particularly of commercially valuable fish (Rogers 1994 and references therein). Onehypothesis to account for this higher abundance is that there may be higher densities of prey items above seamounts because of higher productivity caused by upwelling due to Taylor column formations, which may be particularly important in oligotrophic waters (Boehlert and Genin 1987; Dower et al. 1992; Genin and Boehlert 1985; Rogers 1994). There has been evidence of increased primary productivity over seamounts. High concentrations of chlorophyll A and ATP have been found over seamounts in several studies (Dower et al. 1992; Genin and Boehlert 1985; Lophukin 1986). In those instances, however, the high concentrations were found to be temporary and so thought to be insufficient to explain the abundance of fish and zooplankton around seamounts (Boehlert and Genin 1987; Rogers 1994). One exception was a study conducted at Cobb seamount where enhanced productivity was found to be persistent, although this may be due to the fact that the summit of Cobb penetrates into the euphotic zone (Dower et al. 1992; Rogers 1994). Alternatively, the high abundance of fish at seamounts has been hypothesized to be due to plankton that becomes trapped over seamounts as they are advected over seamounts during their vertical migration (Genin et al. 1988; Isaacs and Schwartzlose 1965; Rogers 1994). Also, certain life history characteristics may be responsible for the large fish aggregations at seamounts, as is the case for the blue ling (Magnusson and Magnusson 1995).

The fauna found on seamounts depend upon the type of habitat. Seamounts are predominantly hard substrate due to their volcanic origin, although seamounts with large flat summits, guyots, are often covered by soft sediments, primarily biogenic sediments such as foraminiferan sands but also sediments of volcanic, lithogenic, and authigenic origin (Rogers 1994). The hard substrata on seamounts are dominated by suspension feeders, mainly gorgonians, zoanthids, antipatharian corals, actinarians, pennatulids, hydroids, sponges, ascidians, and crinoids. The large suspension feeders provide important habitat for smaller, mobile invertebrates (Samadi et al. 2007). Suspension 
feeders accounted for $52 \%$ of a global compilation of benthic seamount species(Wilson and Kaufmann 1987). When broken down by region these values fall with suspension feeders accounting for $15 \%$ in the Eastern Pacific, $27 \%$ on the Tasmanian seamounts, and $22 \%$ on the Howe and Norfolk Ridge in the southwest Pacific (Parin et al. 1997; Samadi et al. 2007). This may be due to the fact that suspension feeders often need hard substrate on which to attach and also a current regime that will provide food as well as prevent sedimentation of the area. Several studies have found that there is a higher density of suspension feeders on areas receiving the greatest flow, such as along the rims of peaks and on the crests of summits (Genin et al. 1986; Rogers 1994).

A survey of soft sediment on two Pacific seamounts found the soft sediment infauna dominated by polychaetes, peracarid crustaceans, aplacophoran, bivalve and gastropod molluscs, sipunculans, nemerteans, and oligochaetes. The meiofauna are dominated by nematodes and harpacticiod copepods with ostracods, loriciferans, and kinorhynchs also being present while the eipfunana is dominated by xenophyophores (Levin et al. 1986; Levin and Thomas 1988; Levin and Thomas 1989). The distribution of fauna in the soft sediment habitat showed an inverse relationship with flow however, with faunal density decreasing with increasing flow (Levin and Thomas 1989).

A recent survey of Bear seamount of the New England Seamount chain in the Atlantic Ocean has described the seamount fauna in the Northwest Atlantic, one area that was often neglected in reviews of seamount fauna (Moore et al. 2003). There were 115 species of fish including some thought to occur only in the eastern Atlantic indicating that this seamount chain may serve as a westward route of dispersal for eastern Atlantic fauna. There were also 26 species of cephalopods, 46 species of crustaceans and a large number of invertebrates spanning 87 species and 10 phyla.

One of the most important fauna supported by seamounts are fish. The concentration of commercially valuable fish in particular, such as orange roughy, hoki, oreosomatids, Patagonian toothfish, and pelagic armourheads has been well documented (Rogers 1994 and references therein; (Koslow et al. 2000b). 
One factor that makes the study of seamounts so important is that along with the high faunal abundances supported by seamounts, they have also been shown to be areas with high degrees of endemism indicating that they may serve as areas of speciation or refugia for relict populations (Rogers 1994; Rogers 2004). Wilson and Kaufman (1987) estimated that $12 \%$ of all fish found on seamounts were endemic while the invertebrate fauna was estimated to have a $15 \%$ level of endemism. The level of fish endemism on the Hawaiian and Emperor seamount chains have been shown to be 17\% (Stocks 2003) while a study at the Great Meteor seamount found fish endemism to be 9\% (Fock et al. 2002). Koslow et al. (2001) found higher levels on the seamounts off Tasmania, which had endemism levels of 35\%. De Forges et al. (2000) found that 29-34\% of the macroand megafauna of seamounts found in the Tasman Sea and southeast Coral Sea were new to science and potential seamount endemics. Even higher rates were found on the Nasca and Sala-y-Gomez seamount chains off the coast of Chile, which had endemism levels for fish of 44\% and 52\% for invertebrates (Parin et al. 1997).

These high levels of endemism are not unique to large and isolated seamounts as would be expected. The Tasmanian seamounts have an average height of $400 \mathrm{~m}$ (Koslow 2001) and seamounts with heights of only 100-350 m near the Chatham Rise have rates of endemism of $15 \%$ (Rowden et al. 2002). This would suggest that seamounts of many sizes serve as potential area of speciation where such high levels of endemism can occur. However recent studies have suggested that these high rates of endemism may be overestimates due to poorly understood species distributions and inadequate sampling (Mcclain 2007; O'hara 2007).

\section{Seamount Population Connectivity}

Related to the issue of endemism is the degree of isolation of seamounts. There have been several studies looking at the connectivity between populations on different seamounts with varying results. A number of different types of studies have shown no isolation between seamounts. Studies of the lightfish, Maurolicus muelleri (Gmelin), 
found that the population at the Southeast Hancock Seamount was made up primarily of juveniles and that there were not enough adults to support a self-sustaining population, indicating possible recruitment from the southern Emperor seamount populations (Boehlert et al. 1994). Another study also found that there was no increase of concentrations of larval fish of seamount-associated species over seamounts versus away from a seamount (Boehlert 1988). Tagging studies of individual yellowfin and bigeye tuna also found that they moved from seamount areas to non-seamount areas (Itano and Holland 2000; Sibert et al. 2000). Genetic studies on fish have also found that there are no differences between different populations found on neighboring seamounts and adjacent non-seamount areas (Bolsch et al. 1993; Borets 1979). Although these studies have shown a lack of isolation between studies, they primarily target large fishes that would be the most mobile of all seamount fauna.

Alternatively, there have been studies that have shown that seamount fauna populations are isolated. The observance of the high degree of endemism is itself an indication of the isolation of seamounts. This is shown through the limited number of species shared by even adjacent seamounts, as was found for adjacent New Caledonian seamounts, which shared only $21 \%$ of species sampled from them(De Forges et al. 2000).

Several studies have also looked at the morphological differences between populations found on different seamount and non-seamount areas. Boehlert et al. (1994) found that the Southeast Hancock Seamount population of the adult lightfish, Maurolicus muelleri (Gmelin), differed in the number of gillrakers from Japanese populations. Wilson and Kaufman (1987) found similar morphological differences in Northeast Atlantic seamount populations of the frostfish, Lepidopus caudatus with distinct northern seamount and southern seamount morphotypes. Similar results were found between the different colonies of the coral Ellisella flagellum on the Great Meteor Seamount and on the Josephine Seamount (Grasshoff 1972).

The life histories of seamount fauna may also be an important factor influencing the amount of dispersal between seamounts. A study on the larval durations of 
invertebrate and fish fauna on Cobb seamount found that there was a higher proportion of species with short or no larval duration suggesting adaptation for local retention of larvae (Parker and Tunnicliffe 1994).

Genetic studies have also shown differentiation between seamount and nonseamount populations. A study of the deep-sea amphipod Eurythenes gryllus found that individuals from the summit of Horizon Guyot were genetically distinct from individuals near the base of the guyot and at other deep-sea sites (Bucklin et al. 1987). It was further found that there was genetic homogeneity of E. gryllus populations within the same depth zone at the scale of ocean basins, but genetically distinct populations at different depths, indicating that depth may be an important factor influencing population structure of seamount fauna (France and Kocher 1996). Most other genetic studies have focused on deep-sea corals. Studies of deep-sea octocorals have found low levels of sequence divergence in mitochondrial $16 \mathrm{~S}$ and COI genes, possibly due to the presence of the $\mathrm{mt} M S H$ gene, a mitochondrial mismatch-repair system. Although distinguishing interspecific differences, these molecular markers are not sensitive enough to determine intraspecific differences and so may not detect recent speciation events and cannot address the issues of intraspecific population connectivity and endemism(France and Hoover 2002; France et al. 1996). A more recent study of deep-sea bamboo coral (Keratoisidinae) found high species diversity in the southwest Pacific using two gene regions of the mitochondrial genome, an insertion/deletion region of the ribosomal large subunit $16 \mathrm{~S}$ and a non-coding region between COI and COII, however these molecular markers were also found to be slow-evolving gene regions limiting their ability to address the issues of endemism and population dispersal (Smith et al. 2004). A study of the coral Corallium launense using microsatellites found low but significant levels of population differentiation among several seamounts and islands in the Hawaiian Archipelago(Baco and Shank 2005). A study of invertebrates from the Norfolk seamounts in the Southwest Pacific found no genetic structure for the two galatheid crabs Munida thoe and Munida zebra, the two chirostylid crab Eumunida species and one plaktotrophic gastropod Sassia remensa, but significant structure for the non-planktotrophic gastropod Nassaria 
probelmatica (Samadi et al. 2006). These studies suggest that there is some degree of isolation of seamounts depending on the taxa studied.

There are several characteristics of seamounts that may be important factors that facilitate or prevent dispersal of seamount fauna. They are unique hard substrate habitats due to their formation from volcanic processes, making them isolated islands within the soft-sediment habitat that dominates the deep-sea benthos. Fauna dependent upon hard substrate would be required to travel across this distance to find other suitable habitat to disperse to and settle on making distance a factor to consider. This would also be dependent upon the geologic history of the seamount, whether they were formed as a chain due to off ridge-axis volcanism such as mantle plumes, in clusters due to on-axis volcanism, or even as solitary seamounts due to tectonic shifts. Also, the morphology of the seamounts themselves can prevent dispersal. With their relatively shallow summits and steep sides, dispersing fauna my not be able to overcome these physical barriers. The hydrographic regime of the region may also have an important role as seamounts have been shown to influence the currents around them, both in ways that could isolate seamounts, such as through the formation of Taylor columns and isolating gyres(Ezer 1994; Mullineaux and Mills 1997; Qiu 1994; Rogers 1994; Taylor 1917) and in ways that could facilitate dispersal, as in deflecting currents and having them run along the axis of a seamount chain possibly serving as a mode of dispersal for larvae and adults(Ezer 1994; Hogg et al. 1986; Qiu 1994; Roden and Taft 1985; Roden et al. 1982; Rogers 1994; Vastano et al. 1985; Vastano and Warren 1976).

At the same time, seamounts have been suggested to act as "stepping-stones" and important for trans-oceanic dispersal of organisms and ocean biodiversity (Hubbs 1959; Rogers 1994). Being unique hard substrate habitats dispersed throughout the world's oceans, they may be key links between different biogeographic provinces within the oceans. Seamounts are often formed in chains perpendicular to coastlines and mid-ocean ridges. These may be key faunal passages for dispersal as was suggested when several fish species previously thought to occur only in the eastern Atlantic were discovered at 
Bear seamount, one of the westernmost seamounts in the North Atlantic (Moore et al. 2003).

\section{North Atlantic Seamounts}

The North Atlantic seamounts involved in this study include three seamount groups, the New England seamount chain, the Corner Rise seamounts and Muir seamount. The geology of the New England seamount chain and its interaction with the hydrographic regime in the North Atlantic has been studied in the past several decades; however, its biological fauna has been mostly ignored (Moore et al. 2003). It is the longest seamount chain in the North Atlantic consisting of about 35 major peaks with elevations ranging from $400-4000 \mathrm{~m}$. The seamounts generally have steep sides and a thin sedimentary cover providing both hard and soft substrates. The seamount chain extends $1200 \mathrm{~km}$ along a northwest-southeast direction, beginning with Bear seamount, located on the continental slope south of the Georges Bank, and ending with Nashville seamount at the end of the Bermuda Rise (Zheng and Arkani-Hamed 2002). The Corner Rise seamounts are a cluster of seamounts located between the Mid-Atlantic Ridge and the New England seamount chain (Figure 1). The Corner Rise seamounts are located approximately $300 \mathrm{~km}$ east of the New England seamounts and consists of about 50 seamounts (Cairns 2007). Additionally, Muir seamount, located northeast of Bermuda, is an isolated seamount. 


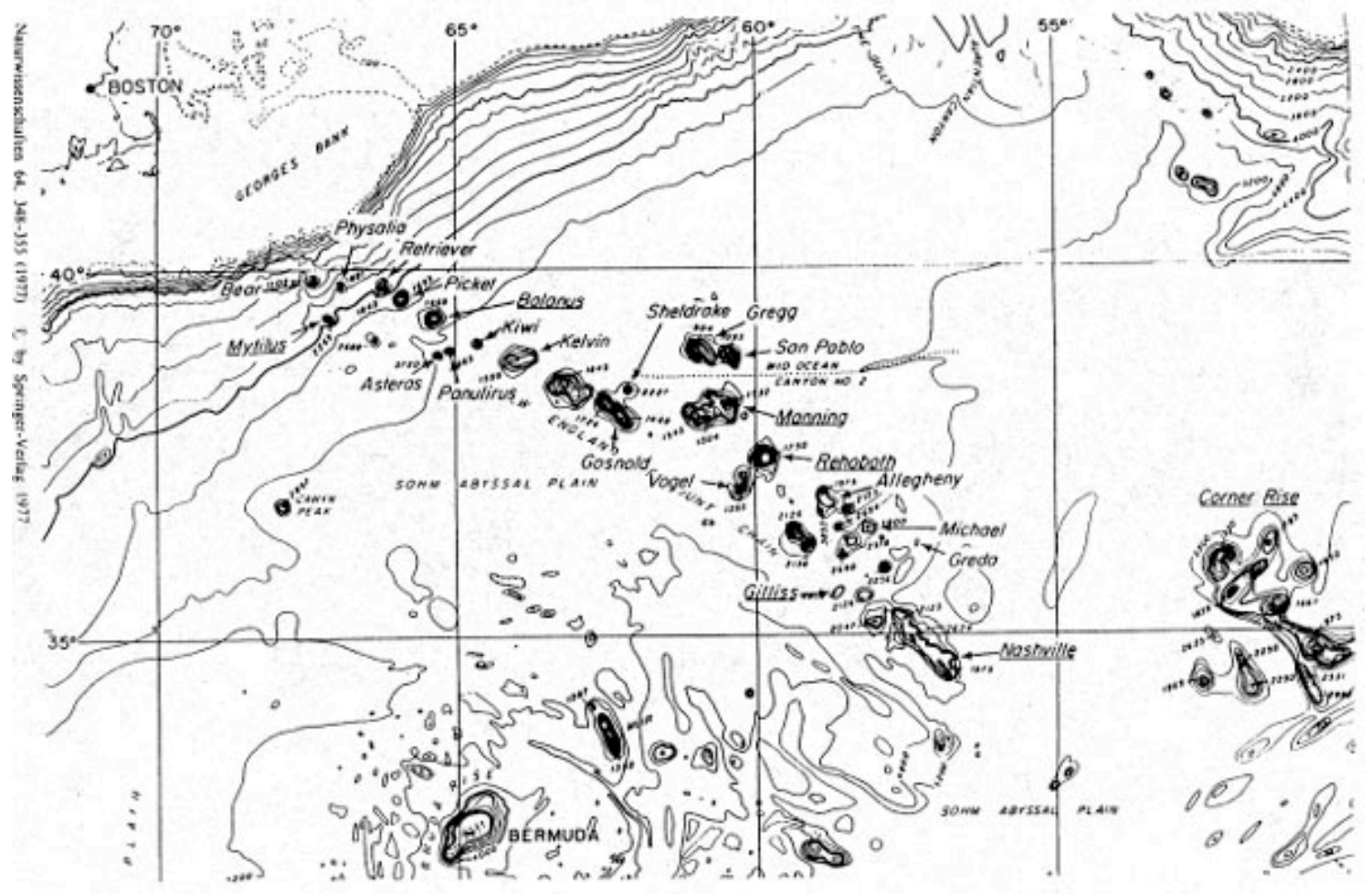

Figure 1. Map of seamounts in the North Atlantic (from Houghton et al. 1977).

The origin of the New England seamount chain is in debate (Zheng and ArkaniHamed 2002). One theory is that the seamounts were formed by volcanism along a fracture zone. A more widely-accepted hypothesis is that the seamount chain was formed as a result of the movement of the North Atlantic plate over the Great Meteor mantle plume. This scenario is thought to explain the formation of not only the New England seamount chain but also the formation of the Corner Rise seamounts and even portions of the White Mountains in New Hampshire (Duncan and Clague 1985; Sleep 1990; Zheng and Arkani-Hamed 2002). The ages of the seamounts have also been investigated through ${ }^{40} \mathrm{Ar} /{ }^{39} \mathrm{Ar}$ isotope dating, with the oldest New England seamount, Bear, in the northwest estimated to have been formed 103 mya and the youngest New England seamount, Nashville, estimated to be formed 82 mya. The Corner Rise seamounts are estimated to be formed approximately 75 mya (Duncan 1984; Zheng and Arkani-Hamed 2002) (Figure 2). 


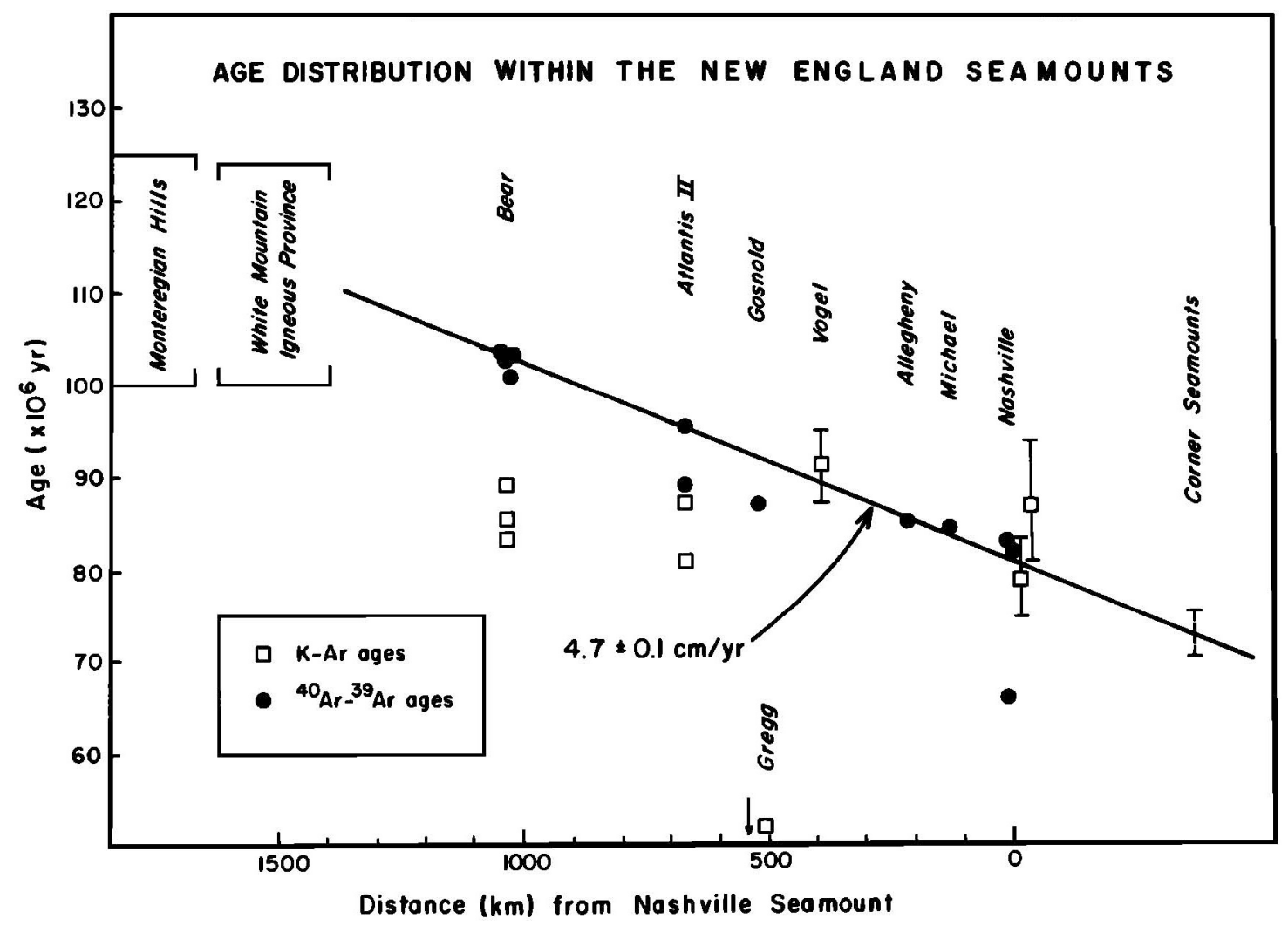

Figure 2 Age distribution of the New England seamounts based on Ar isotope dating. Figure modified from Duncan 1984).

The seamounts in the North Atlantic have also been shown to interact with the hydrography of the region as well. Modeling studies have shown that the seamounts may affect the currents in the region, in particular the Gulf Stream, which bisects the New England seamount chain and produces gyres with circulations occurring in opposite directions acting as a possible barrier between the western and eastern seamaints within the chain. Hypothesized gyres around the Corner Rise seamounts may also serve as isolating mechanisms (Ezer 1994; Qiu 1994) (Figure 3). 


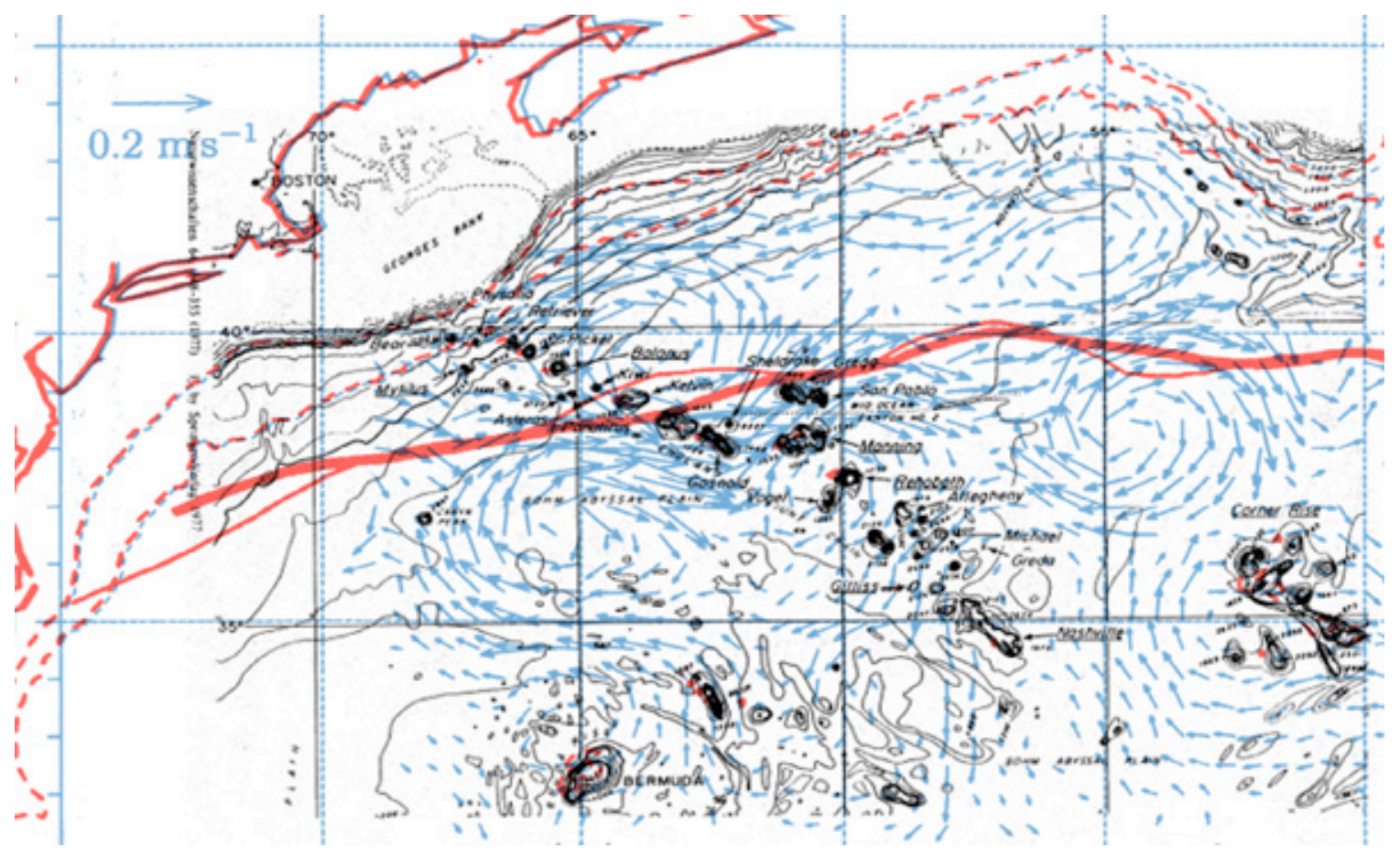

Figure 3. Predicted flow (blue arrows) of the Gulf Stream at $3000 \mathrm{~m}$ depth (mean flow in solid red) across the New England Seamounts, calculated from surface to deep water modeling studies. Figure modified from Houghton et al., 1977; Qiu, 1994.

The interacting biological, physical, and hydrological characteristics of seamounts and seamount communities indicate that they are important features in marine biodiversity, biogeography, and evolution. Seamounts may be significant links between different biogeographic provinces within the oceans acting as "stepping-stones" and playing an important part in the trans-oceanic dispersal of organisms as key faunal passages for dispersal (Hamilton 1956; Hubbs 1959; Rogers 1994). With the increasing pressures of deep-sea fisheries destroying a potentially vital link within ocean systems it is all the more important to assess the population dynamics of seamount fauna. This will not only provide a greater understanding of ocean biogeography and deep-sea biodiversity but also be an important step in guiding conservation efforts to protect the vulnerable seamount fauna. With the collapse of traditional continental shelf and pelagic 
fisheries and the improvement of fishing technology, deep-sea fisheries have grown and have contributed 800,000 to 100,000 tons annually to global fish landings since 1964 (Clark 2001; Koslow et al. 2000a). Deep-sea fishing techniques such as trawling and long-line fishing have been shown to destroy benthic habitat, in particular the coral habitat upon which many invertebrates live (Kaiser 1998; Probert 1999; Probert et al. 1997; Thrush et al. 1998). With expanding deep-sea fisheries and the concurrent destruction of seamount habitat, particularly of the live and fossil corals, conservation efforts for these areas have increased. One example is the proposal for the precautionary closure of four seamount areas in the North Atlantic to deep-sea fisheries, which include portions of the Corner and New England seamounts (Nafo 2006). Understanding the extent of connectivity between seamounts and seamount areas will aid in the creation of marine protected areas and future conservation and policy efforts. One way to do this is to assess the level of genetic connectivity between seamount populations. Genetic connectivity has been defined as the exchange of individuals between subpopulations that contribute to the local gene pool (Hedgecock et al. 2007). Such a strategy has been advocated for the conservation of coral reef communities (Palumbi 2003; Richards et al. 2007).

\section{Goals of this study}

The mechanism of faunal dispersal across ocean basins is one of the key unknowns in our understanding of the present-day biogeography and biodiversity of deep-sea fauna. It has been proposed that seamounts play several important roles in faunal evolution, acting as regional centers of speciation, "stepping-stones" for dispersal across ocean basins and boundary currents, and refugia for populations with shrinking ranges. Seamounts have also been noted for their high degree of endemism and are areas of high production that support abundant faunal assemblages.

The goals of my dissertation were to further our understanding of the role seamounts play in marine biodiversity and biogeography. This study was focused on the 
North Atlantic seamounts, including the New England Seamount chain, the Corner Rise seamounts, and Muir seamount. I employed videographic analyses to characterize the fauna and faunal assemblages found on seamounts, including quantifying specific hostassociate relationships, and to identify patterns of faunal distribution on the seamounts and the factors important for creating, structuring, and maintaining these patterns. I then focused on a particular faunal group, specifically ophiuroids, to see whether these broad community patterns were discernible on a genetic level and may have an evolutionary impact. I assessed the usefulness of the $16 \mathrm{~S}$ mtDNA gene as a genetic "barcode" to identify ophiuroid species in order to assess the diversity of ophiuroids on seamounts and to confirm preliminary morphospecies identifications of four target species for a population genetic study. A comprehensive investigation of four ophiuroid species with potentially varying life histories found on the North Atlantic seamounts was employed to assess the genetic population structure across the North Atlantic region and also the level of faunal dispersal and gene flow. 


\section{REFERENCES}

BACO, A. R., and T. M. SHANK. 2005. Population genetic structure of the Hawaiian precious coral Corallium lauuense (Octocorallia: Coralliidae) using microsatellites, p. 663-678. In A. Freiwald and J. M. Roberts [eds.], Cold-water Corals and Ecosystems. Springer-Verlag.

BATIZA, R. 2001. Seamounts and Off-Ridge Volcanism, Encyclopedia of Ocean Sciences.

BoeHLERT, G. W. 1988. Current-topography interactions at mid-ocean seamounts and the impact on pelagic ecosystems. Geojournal 16: 45-52.

Boehlert, G. W., and A. GENIN. 1987. A review of the effects of seamounts on biological processes, p. 319-334. In B. Keating, G. J. Fryer, R. Batiza and G. W. Boehlert [eds.], Seamounts, Islands, and Atolls. Geophysical Monograph. American Geophysical Union.

Boehlert, G. W., C. D. Wilson, and K. Mizuno. 1994. Populations of the sternoptychid fish Maurolicus muelleri on seamounts in the Central North Pacific. Pacific Science 48: 57-69.

Bolsch, C. J. S., N. G. ElliotT, and R. D. WARD. 1993. Enzyme variation in southeastern Australian samples of the blue-eye or deepsea trevalla, Hyperoglyphe antarctica Carmichael 1818 (Teleostei: Stromateoidei). AUST. J. MAR. FRESHWAT. RES. 44: 687-697.

Borets, L. A. 1979. The population structure of the boarfish Pentaceros richardsoni (Smith) from the Emperor Seamounts and the Hawaiian Ridge. Vopr. Ikhtiol. 19: 402-407.

Brewin, P. E., K. I. Stocks, and G. Menezes. 2007. A history of seamount research, p. 41-61. In T. J. Pitcher et al. [eds.], Seamounts: Ecology, Fisheries, \& Conservation. Fish and Aquatic Resources Series. Blackwell Publishing.

BuCKLIN, A., R. R. Wilson, JR., and K. L. SMITH, JR. 1987. Genetic differentiation of seamount and basin populations of the deep-sea amphipod Eurythenes gryllus. Deep-Sea Research (Part I, Oceanographic Research Papers) 34: 1795-1810.

CAIRNS, S. D. 2007. Studies on the western Atlantic Octocorallia (Gorgonacea: Primnoidae). Part 8: New records of Primnoidae form the New England and Corner Rise Seamounts. Proceedings of the Biological Society of Washington 120: 243-263.

Clark, M. 2001. Are deepwater fisheries sustainable? -- the example of orange roughy (Hoplostethus altanticus) in New Zealand. Fisheries Research 51: 123-135.

De Forges, B. R., J. A. Koslow, and G. C. B. Poore. 2000. Diversity and endemism of the benthic seamount fauna in the southwest Pacific. Nature [Nature]. Vol.405: 944-947.

Dower, J., H. FreELAND, and K. JUNIPER. 1992. A strong biological response to oceanic flow past Cobb Seamount. Deep-Sea Research 39: 1139-1145. 
DUNCAN, R. A. 1984. Age progressive volcanism in the New England seamounts and the opening of the central Atlantic ocean. Journal of Geophysical Research 89: 99809990.

Duncan, R. A., and D. A. Clague. 1985. Pacific Plate motion recorded by linear volcanic chains. Plenum Press, New York, NY, United States (USA).

Epp, D., and N. C. SмOOT. 1989. Distribution of seamounts in the North Atlantic. Nature 337: 254-257.

EzER, T. 1994. On the interaction between the Gulf Stream and the New England Seamount Chain. Journal of Physical Oceanography 24: 191-204.

Fock, H., F. Uiblen, F. Koster, and H. VON WeSTERNHAGEN. 2002. Biodiversity and species-environment relationships of the demersal fish assemblage at the Great Meteor Seamount (subtropical NE Atlantic), sampled by different trawls. Marine Biology 141: 185-199.

FranCE, S. C., and L. L. HoOver. 2002. DNA sequences of the mitochondrial COI gene have low levels of divergence among deep-sea octocorals (Cnidaria: Anthozoa). Hydrobiologia 471.

France, S. C., and T. D. Kocher. 1996. Geographic and bathymetric patterns of mitochondrial 16S rRNA sequence divergence among deep-sea amphipods, Eurythenes gryllus. Marine Biology 126: 633-643.

France, S. C., P. E. Rosel, J. E. Agenbroad, L. S. Mullineaux, and T. D. Kocher. 1996. DNA sequence variation of mitochondrial large-subunit rRNA provides support for a two-subclass organization of the Anthozoa (Cnidaria). Molecular Marine Biology and Biotechnology 5: 15-28.

FRYER, P., and G. J. FRYER. 1987. Origins of nonvolcanic seamounts in a forearc an environment. In B. Keating, P. Fryer, R. Batiza and G. W. Boehlert [eds.], Seamounts, Islands, and Atolls. Geophysical Monograph. American Geophysical Union.

Genin, A., and G. W. BoeHLERT. 1985. Dynamics of temperature and chlorophyll structures above a seamount: an oceanic experiment. Journal of Marine Research 43: 907-924.

Genin, A., P. K. Dayton, P. F. Lonsdale, and F. N. Spiess. 1986. Corals on seamount peaks provide evidence of current acceleration over deep-sea topography. Nature 322: $59-61$.

Genin, A., L. R. HAURY, and P. GREENBLATt. 1988. Interactions of migrating zooplankton with shallow topography: predation by rockfishes and intensification of patchiness. Deep-Sea Research 52: 151-175.

GRASSHOFF, M. 1972. The Gorgonaria of the eastern North Atlantic and the Mediterranean. I. Family Ellisellidae (Cnidaria: Anthozoa). Results of the 'Atlantic Seamount Cruises 1967' with R. V. 'Meteor'. Meteor Forschungsergeb. 10: 73-87.

Hamilton, E. L. 1956. Sunken islands of the Mid-Pacific Mountains. Geological Society of America Memoir 64.

HedGeCock, D., P. H. BARber, and S. EdMANDS. 2007. Genetic approaches to measuring connectivity. Oceanography 20: 70-79. 
HoGG, N. G. 1973. On the stratified Taylor column. Journal of Fluid Mechanics 58: 517537.

HogG, N. G., R. S. Pickart, R. M. Hendry, and W. J. SMethiE, JR. 1986. The Northern Recirculation Gyre of the Gulf Stream. Deep-Sea Research (Part I, Oceanographic Research Papers) 33: 1139-1165.

HuBBS, C. L. 1959. Initial Discoveries of Fish Faunas on Seamounts and Offshore Banks in the Eastern Pacific. Pacific Science 13: 311-316.

IsAaCS, J. D., and R. A. SchWARTZLOSE. 1965. Migrant sound scatterers: interactions with the seafloor. Science 150: 1810-1813.

ITANO, D. G., and K. N. HolLAND. 2000. Movement and vulnerability of bigeye (Thunnus obesus) and yellowfin tuna (Thunnus albacares) in relation to FADs and natural aggregation points. Aquatic Living Resources 13: 213-223.

KAISER, M. J. 1998. Significance of Bottom-Fishing Disuturbance. Conservation Biology 12: $1230-1235$.

KosLOw, J. A. 2001. Fish stocks and benthos of seamounts. BfN Skripten 43.

Koslow, J. A., G. W. Boehlert, J. D. M. Gordon, R. L. Haedrich, P. Lorance, and N. PARIN. 2000a. Continental slope and deep-sea fisheries: implications for a fragile ecosystem. ICES Journal of Marine Science 57: 548-557.

Koslow, J. A., J. D. Boehlert, M. Gordon, R. L. HAEDrich, P. Lorance, and N. PARIN. 2000b. Continental slope and deep-sea fisheries: implications for a fragile ecosystem. ICES Journal of Marine Science 57: 548-557.

Levin, L. A., D. J. DeMaster, L. D. MCCAnN, and C. L. Thomas. 1986. Effects of giant protozoans (class: Xenophyophorea) on deep-seamount benthos. Marine Ecology Progress Series 29: 99-104.

LeVIn, L. A., and C. L. THOMAS. 1988. The ecology of xenophyophores (Protista) on eastern Pacific seamounts. Deep-Sea Research 12.

---. 1989. The influence of hydrodynamic regime on infaunal assemblages inhabiting carbonate sediments on central Pacific Seamounts. Deep-Sea Research 36: 18971915.

LOPHUKIN, A. S. 1986. Distribution of ATP concentration above seamounts in the Atlantic ocean. Oceanology 26: 361-365.

MAgnusson, J. V., and J. MAgNusson. 1995. The distribution, relative abundance, and biology of the deep-sea fishes of the Icelandic slope and Reykjanes Ridge, p. 161199. In A. G. Hooper [ed.], Deep-water Fisheries of the North Atlantic Oceanic Slope. Kluwer Academic Publishers.

MCClaIN, C. R. 2007. Seamounts: identity crisis or split personality? Journal of Biogeography 34: 2001-2008.

Menard, H. W. 1964. Marine Geology of the Pacific. McGraw-Hill.

Moore, J. A. and others 2003. Biodiversity of Bear Seamount, New England Seamount chain: Results of exploratory trawling. Journal of Northwest Atlantic Fishery Science 31: 363-372.

MullineauX, L. S., and S. W. Mills. 1997. A test of the larval retention hypothesis in seamount-generated flows. Deep-Sea Research 44: 745-770. 
NAFO. 2006. Proposal on precautionary closure to four seamount areas based on the ecosystem approach to fisheries. In N. A. F. Organization [ed.].

O'HARA, T. D. 2007. Seamounts: centres of endemism or species richness for ophiuroids? Global Ecology and Biogeography 16: 720-732.

PALUMBI, S. R. 2003. Population genetics, demographic connectivity, and the design of marine reserves. Ecological Applications 13: 146-158.

Parin, N. V., A. N. Mironov, and K. N. Nesis. 1997. Biology of the Nazca and Sala y Gomez Submarine Ridges, an Outpost of the Indo-West Pacific Fauna in the Eastern Pacific Ocean: Composition and Distribution of the Fauna, its Communities and History. Advances in Marine Biology 32: 145-242.

PARKER, T., and V. TunNiClifFE. 1994. Dispersal Strategies of the Biota on an Oceanic Seamount: Implications for Ecology and Biogeography. Biological Bulletin, Marine Biological Laboratory, Woods Hole [Biol. Bull. Mar. Biol. Lab. Woods Hole] 187.

Probert, P. K. 1999. Seamounts, sanctuaries, and sustainability: moving towards deepsea conservation. Aquatic Conservation: Marine and Freshwater Ecosystems 9: 601-605.

Probert, P. K., D. G. MCKnight, and S. L. Grove. 1997. Benthic invertebrate bycatch from a deep-water trawl fishery, Chatham Rise, New Zealand. Aquatic Conservation: Marine and Freshwater Ecosystems 7: 27-40.

QIU, B. 1994. Determining the mean Gulf Stream and its recirculations through combining hydrographic and altimetric data. Journal of Geophysical Research 99: 951-962.

Richards, V. P., J. D. Thomas, M. J. Stanhope, and M. S. Shivj. 2007. Genetic connectivity in the Florida reef system: comparative phylogeography of commensal invertebrates with contrasting reproductive strategies. Mol Ecol16: 139-157.

Richardson, P. L. 1980. Anticyclonic eddies generated near Corner Rise seamounts. Journal of Marine Research 38: 673-686.

Roden, G. I., and B. A. TAFT. 1985. Effect of the Emperor seamount on the mesoscale thermohaline structure during the summer of 1982. Journal of Geophysical Research 90: 839-855.

Roden, G. I., B. A. TAFT, and C. C. EBBesmeYer. 1982. Oceanographic aspects of the Emperor seamounts region. Journal of Geophysical Research 87: 9537-9552.

Rogers, A. D. 1994. The biology of seamounts. Advances in Marine Biology 30: 305350.

---. 2004. The Biology, Ecology, and Vulnerability of Seamount Communities. International Union for Conservation of Nature \& Natural Resources.

Rowden, A. A., S. O'SHEA, and M. R. CLARK. 2002. Benthic biodiversity of seamounts on the northwest Chatham Rise, p. 21, Marine Biodiversity Biosecurity Report Ministry of Fisheries.

Samadi, S., L. Bottan, E. Macpherson, B. Richer de Forges, and M. Boisselier. 2006. Seamount endemism questioned by the geographic distribution and 
population genetic structure of marine invertebrates. Marine Biology 149: 14631475.

SAmAdI, S., T. Schlacher, and B. R. DE ForgeS. 2007. Seamount benthos, p. 119-140. In T. J. Pitcher et al. [eds.], Seamounts: Ecology, Fisheries, \& Conservation. Fish and Aquatic Resources Series. Blackwell Publishing.

Sibert, J., K. N. Holland, and D. G. ITANO. 2000. Exchange rates of yellowfin and bigeye tunas and fishery interaction between Cross seamount and near-shore FADs in Hawaii. Aquatic Living Resources 13: 225-232.

SLEEP, N. H. 1990. Monteregian hotspot track: a long-lived mantle plume. Journal of Geophysical Research 95: 21,983-921,990.

Smith, P. J., S. M. McVeagh, J. T. Mingoia, and S. C. France. 2004. Mitochondrial DNA sequence variation in deep-sea bamboo coral (Keratoisidinae) species in the southwest and northwest Pacific Ocean. Marine Biology 144: 253-261.

Stocks, K. I. 2003. SeamountsOnline, a Biogeographic Information System for Seamounts, Frontiers in Biogeography, the Inaugural Meeting of the International Biogeography Society.

TAYLOR, G. I. 1917. Motion of solids in fluids when the flow is not irrotational. Proceedings of the Royal Society A 93: 99-113.

Thrush, S. F. and others 1998. Disturbance of the Marine Benthic Habitat by Commercial Fishing: Impacts at the Scale of the Fishery. Ecological Adaptations 8: 866-879.

Vastano, A. C., D. E. Hagen, and G. J. McNally. 1985. Lagrangian observations of surface circulation at the Emperor seamount chain. Journal of Geophysical Research 90: 3325-3331.

Vastano, A. C., and B. A. WArren. 1976. Perturbations of the Gulf Stream by Atlantis II seamount. Deep-Sea Research 23: 681-694.

Wilson, R. R., JR., and R. S. KaUfmann. 1987. Seamount biota and biogeography, p. 355-377. In B. H. Keating, P. Fryer, R. Batiza and G. W. Boehlert [eds.], Seamounts, Islands, and Atolls. Geophysical Monograph Series. American Geophysical Union.

ZHENG, Y., and J. ARKANI-HAMED. 2002. Rigidity of the Atlantic oceanic lithosphere beneath New England seamounts. Tectonophysics 359: 359-369. 


\section{Chapter 2: Fauna-habitat associations and community structure on the New England and Corner Rise Seamounts}

\section{INTRODUCTION}

Seamounts are common geological features of the ocean floor. There are an estimated 100,000 seamounts present throughout the world's oceans found across a wide range of latitudes and depths with diverse geological histories and ages (Kitchingman et al. 2007; Samadi et al. 2007). Many questions remain about the biogeographic features of these distinctive deep-sea habitats, the biological communities they support and the factors important for creating, structuring, and maintaining those communities, and the role they play in marine biodiversity and biogeography (Brewin et al. 2007).

Seamounts are areas of high biodiversity for both pelagic and benthic communities, including commercially valuable fish (Genin and Boehlert 1985; Lophukin 1986; Rogers 1994). The benthic biomass of seamounts is often dominated by large suspension feeders that provide important habitat for smaller, mobile invertebrates (Samadi et al. 2007). In addition to supporting a large abundance of fauna, seamounts are thought to show high degrees of endemism, indicating that they may serve as areas of speciation or refugia for relict populations (Fock et al. 2002; Koslow et al. 2001; Parin et al. 1997; Richer De Forges et al. 2000; Rogers 1994; Wilson and Kaufmann 1987).

Interactions between biological and oceanographic processes at seamounts, such as the deflection of currents, the formation of eddies, or Taylor columns (Ezer 1994; Mullineaux and Mills 1997; Qiu 1994; Richardson 1980; Roden and Taft 1985; Roden et al. 1982; Rogers 1994; Taylor 1917; Vastano et al. 1985; Vastano and Warren 1976), are 
not fully understood and may be an important contributing factor in the biological characteristics of seamounts and their role in marine biodiversity and biogeography.

The interacting biological, physical, and hydrological characteristics of seamounts and seamount communities indicate that they are important factors in marine biodiversity, biogeography, and evolution. Seamounts may be significant links between different biogeographic provinces within the oceans acting as "stepping-stones" and playing an important part in the trans-oceanic dispersal of organisms as key faunal passages for dispersal (Hamilton 1956; Hubbs 1959; Rogers 1994). With expanding deep-sea fisheries and the concurrent destruction of seamount habitat, particularly of the live and fossil corals (Clark 2001; Kaiser 1998; Koslow et al. 2000; Probert 1999; Probert et al. 1997; Thrush et al. 1998), renewed interest in seamounts has grown over the past several years as this increasing threat to seamount habitats and ecosystems makes ascertaining the true role of seamounts and their importance in ocean dynamics all the more urgent.

The North Atlantic seamounts include two seamount groups, the New England seamount chain and the Corner Rise seamounts. The geology of the New England seamount chain and its interaction with the hydrographic regime in the North Atlantic has been studied in the past several decades; however, its biological fauna has been mostly ignored (Moore et al. 2003). It is the longest seamount chain in the North Atlantic consisting of about 35 major peaks with elevations ranging from 400-4000 m. The seamounts generally have steep sides and a thin sedimentary cover providing both hard and soft substrates. The seamount chain extends $1200 \mathrm{~km}$ along a northwest-southeast direction, beginning with the oldest seamount, Bear, located on the continental slope south of the Georges Bank, and ending with the youngest seamount, Nashville, at the end of the Bermuda Rise (Zheng and Arkani-Hamed 2002). They have been thought to have been formed been formed between 103 and 82 mya (Duncan 1984; Zheng and ArkaniHamed 2002). The Corner Rise seamounts are a cluster of seamounts located between the Mid-Atlantic Ridge and the New England seamount chain (Figure 1). The Corner Rise seamounts are located approximately $300 \mathrm{~km}$ east of the New England seamounts 
and consists of about 50 seamounts and are thought to have been formed about 75 mya (Cairns 2007; Duncan 1984; Zheng and Arkani-Hamed 2002).

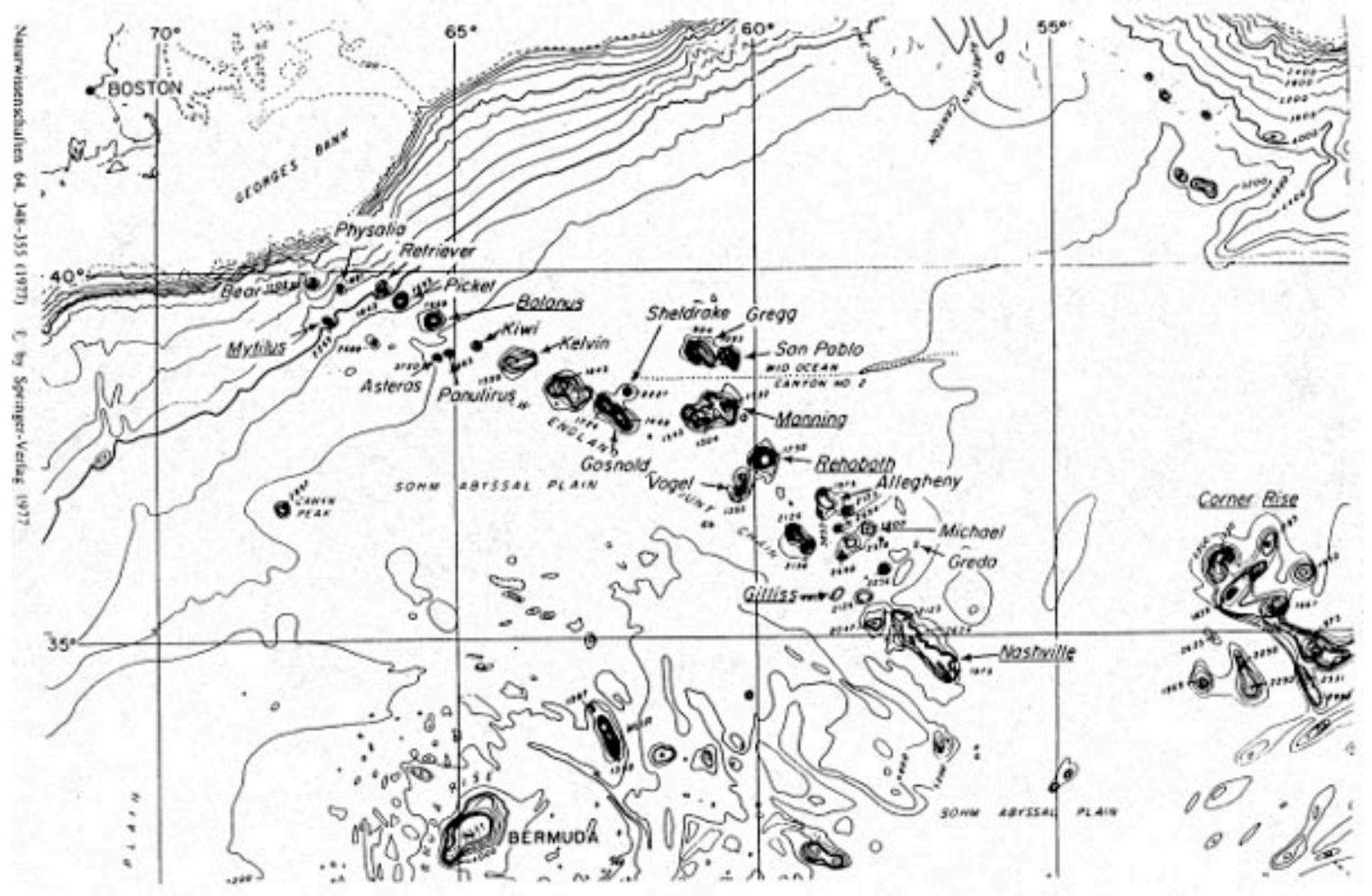

Figure 1. Map of seamounts in the North Atlantic (from Houghton et al. 1977).

The seamounts in the North Atlantic have also been shown to interact with the hydrography of the region as well. Modeling studies have shown that the seamounts may affect the currents in the region, in particular the Gulf Stream, which bisects the New England seamount chain and produces gyres with circulations occurring in opposite directions acting as a possible barrier between the western and eastern seamounts within the chain. Based on these modeling studies, a gyre around the Corner Rise seamounts may serve as an isolating mechanisms (Ezer 1994; Qiu 1994) (Figure 2). 


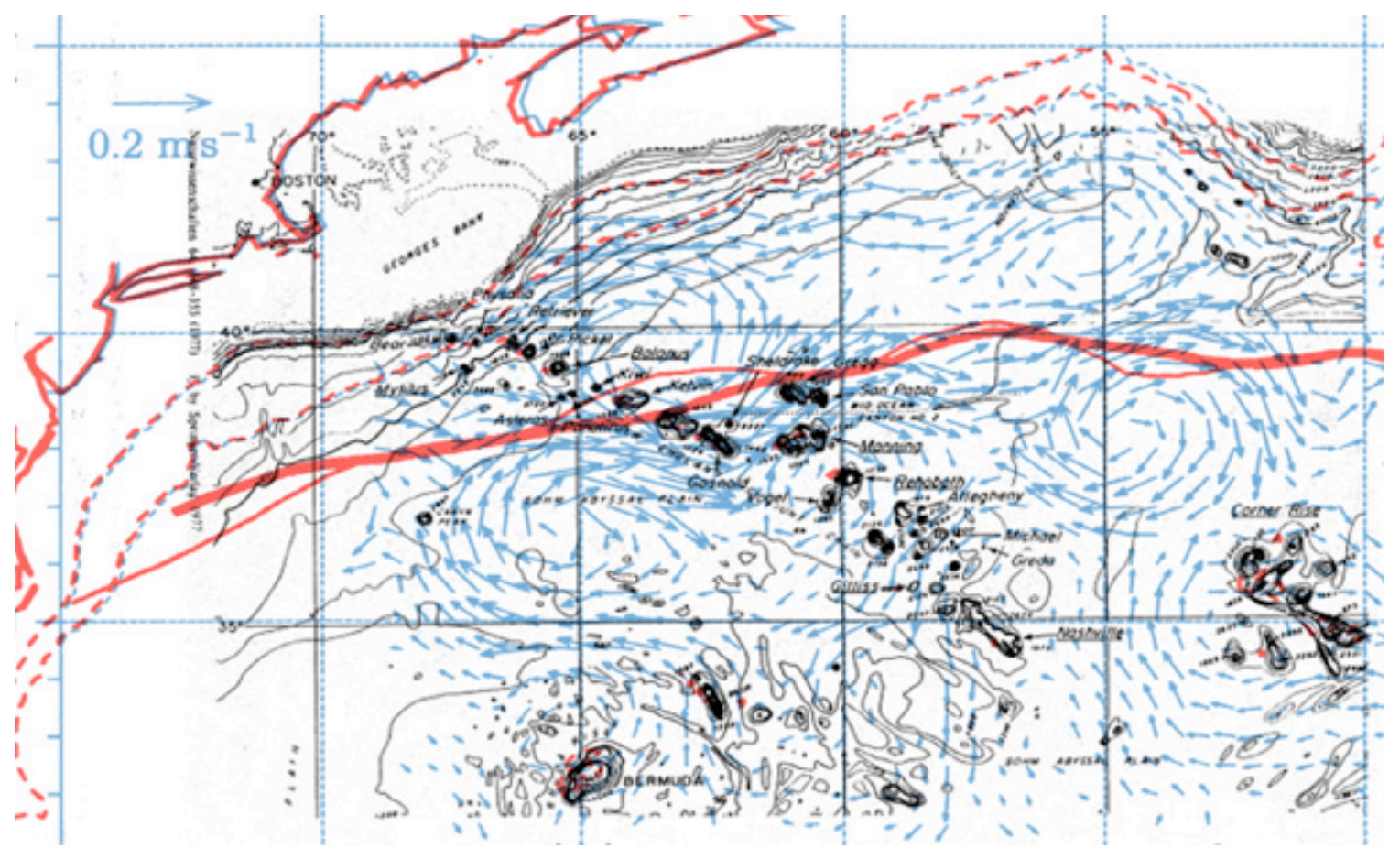

Figure 2. Predicted flow (blue arrows) of the Gulf Stream at 3000m depth (mean flow in solid red) across the New England Seamounts, calculated from surface to deep water modeling studies. Figure modified from Houghton et al., 1977; Qiu, 1994.

Videographic analysis was conducted from dives on the New England and Corner Rise seamounts in order to examine the fauna and communities of the North Atlantic seamounts. The goals of this study are:

1. to characterize the composition and distribution of seamount fauna,

2. to identify potential symbiotic relationships among the fauna,

3. to distinguish the different populations and analyze the similarity of those seamount populations based on community composition and

4. to identify the factors that may be important for structuring these communities and may play a role in maintaining biogeographic boundaries. 
It is expected that the communities between the different seamount chains will be significantly different and that factors of geographic distance, geology, current patterns, and depth may potentially be important for structuring the seamount communities. Also, symbiotic relationships are expected as well, based on previous studies and anecdotal accounts.

\section{MATERIALS AND METHODS}

\section{Video Acquisition}

In August 2005 the Hercules/Argus ROV system was utilized to explore seamounts in the Corner Rise and New England seamount chain aboard the R/V Ronald H. Brown during the Deep Atlantic Stepping Stones research cruise (Table 1). The objectives of the cruise were: to determine the taxonomic composition of seamount communities the biogeographic relationships of corals and associated fauna in the New England and Corner Rise seamounts; to determine the taxonomic composition and distribution of paleo-fauna in order to study climate history in the region; to determine the reproductive state and potential larval strategies of seamount corals; to study colonization dynamics of coral aggregations; to determine the relationship between the demersal nekton to seamount landscape features, and to assess the physical impact of bottom trawling on coral communities.

Multibeam sonar surveys were initially conducted over the target seamounts using the SeaBeam $2120(20 \mathrm{kHz})$ swath bathymetric system aboard the R/V Ronald H. Brown. This system has 151 beams in a $150^{\circ}$ fan-shaped array. These surveys produced detailed bathymetric maps of the target seamounts which were then used to select dive sites. Diving sites were selected in order to cover a variety of depth ranges and region faces of 
the seamounts, as well as areas with steep topography. Consideration was also given to the weather and oceanographic current conditions at the time.

The Hercules/Argus ROV system was then used to survey these seamounts. This system involves two separate vehicles used in tandem. Argus is connected between the ship and Hercules, acting to decouple ship and cable motions from Hercules. The Argus was equipped with three HMI lights with a total of $2000 \mathrm{~W}$ of power and a high definition Insite Pacific Zeus HDTV camera (resolution 1035i), and acted as a lighting and video platform for Hercules.

The ROV Hercules was equipped with four $250 \mathrm{~W}$ incandescent lights (DeepSea Power \& Light Multi-Sea Lite), two 400 W HMI ballasted lights (DSPL), and two Ocean Imaging $130 \mathrm{~W}$-sec strobe lights. Hercules had a variety of camera systems including a high definition Insite Pacific Zeus HDTV camera (resolution 1035i) that provided video and still imagery for analysis, an Insite Pacific Titan tilt-rotate standard definition camera (resolution 480p, NTSC), standard definition utility cameras, and a stereo pair of Prosilica GE1380 pixelfly digital still cameras, one color and one monochrome (resolution 1.4 megapixels, 1360x1024). Video was recorded from the Hercules HD camera in standard definition to DVCam tapes, with select segments recorded to HD tapes.

Hercules was also equipped with a Seabird 2 Micro CTD sensor, an AANDERAA Optode 3830 dissolved oxygen sensor, a Kraft Predator force-reflecting manipulator arm, an ISE Magnum manipulator arm, various adaptors for the manipulator arms for use during collection, insulated bioboxes for sample collection, and two suction pumps. The manipulator arm was modified with cutting blades to aid in the collection of corals.

Image acquisition was achieved with the ROV to touchdown at the deepest target depth and perform transects going upslope. Video and stills were taken throughout the dive to document the landscape and fauna. Corals and other select invertebrates were collected using the manipulator arms and the suction samplers to serve as vouchers, for the observed and collected organisms, to ground-truth results for the video analyses, and 
for future genetic studies. Fossil corals were collected at different depths using the ROV manipulators and modified nets.

Records were made quantifying the seamount fauna using the Horita PC-log tape logging software, which allowed observations to be made in sync with the timecode of the video. Individual morphospecies identification and relative abundances were enumerated, recording all visible fauna, the quantity in which they occurred, and what they were found on, or "substrate" (either soft sediment, hard substrate, the water column, or another organism or object). Species identification were assigned to morphospecies identifications when possible based on either published names (e.g., (Watling 2007), identifications made by "taxonomic assessment" and not published (e.g, in conjunction with Smithsonian Institution and Yale Peabody museum), or current working or tentative identifications by the principal investigators. These records were merged according to time with the navigation data from the ROV Hercules, which provided location and CTD data correlated to our observations.

\section{Video Analysis}

Observation data was standardized by total observations made in a sample bin. Relative abundances were used rather than abundances calculated from area because of the high degree of variability in the altitude of the ROV Hercules and in the magnification of the camera throughout the transects. The data underwent a fourth-root transformation in order to account for differences between rare and abundant fauna. Correlation matrices using the Bray-Curtis similarity index were generated using the fourth-root transformed data and cluster analysis and multidimensional scaling (MDS) were used to determine similarity of biological assemblages based on species composition. The significance of the resulting patterns were tested using the Analysis of Similarity (ANOSIM) routine. In certain cases, the SIMPER routine was used to determine the variables responsible for the average Bray-Curtis dissimilarity between 
groups. The analyses were performed at several scales by grouping the observations into different sample groups:

1. By seamount, examining regional differences between

a. the communities on the Corner Rise and those on the New England seamount chain

b. the communities on the Corner Rise, the eastern New England seamount chain, and the western New England seamount chain

2. By $100 \mathrm{~m}$ depth bins, examining differences in community structure by depth

3. By Transect, examining community differences between local populations

4. By Substrate, to examine differences between

a. abiotic and biotic substrate

b. within abiotic substrate

i. natural and anthropogenic substrate

c. within biotic substrate

i. living and dead substrate

d. within living substrate

i. mobile and sessile substrate

ii. corals and sponges

iii. coral orders

iv. coral families

5. By $100 \mathrm{~m}$ depth bins within a seamount, comparing the influence of the factors of depth and geography, or seamount

An analysis to determine similarity of biological assemblages based on substrate composition was also performed by grouping the observations of substrates into different sample groups: 
6. By seamount, to examine regional differences between

a. the Corner Rise and the New England seamount chain

b. the Corner Rise, the eastern New England seamount chain, and the western New England seamount chain

7. By $100 \mathrm{~m}$ depth bins, to examine for differences by depth

These analyses were performed with the statistical package PRIMER v6 (Clarke and Gorley 2006; Clarke and Warwick 2001).

The analyses were repeated on a dataset with more conservative identifications where morphospecies that were very similar were grouped by genus. This was done in order to determine whether the patterns observed in the initial analysis were similar to those observed at a coarser level of taxonomic identification.

In order to study host-associate relationships, the percent occurrence of all observable organisms was also calculated for each of the different substrates on which they were found that may be a significant host.

\section{RESULTS}

\section{Video Transects}

Five peaks in the Corner Rise and five seamounts in the New England Seamount chain were surveyed and sampled (Figure 3 - Figure 8). Within the New England Seamount chain, three seamounts were from the east of the Gulf Stream and two to the west of the Gulf Stream. A total of 16 dives were conducted. Transects for video analysis began when the ROV Hercules arrived on the seafloor and ended when it left the seafloor at the end of a dive. Transects had an average duration of 13 hours and 10 minutes, an average transect length of $2.83 \mathrm{~km}$, and an average depth coverage of $319 \mathrm{~m}$ 
(Table 1). These dives provided a total of 209 hours and 53 minutes of video footage from the seafloor to analyze.

Table 1. Summary of dive information during the Deep Atlantic Stepping Stones expedition.

\begin{tabular}{|l|l|l|c|c|c|}
\hline Transect & Date & $\begin{array}{l}\text { Seamount } \\
\text { Peak }\end{array}$ & $\begin{array}{c}\text { Bottom } \\
\text { Time }\end{array}$ & $\begin{array}{c}\text { Transect Length } \\
\mathbf{( k m})\end{array}$ & $\begin{array}{c}\text { Depth } \\
\text { Range (m) }\end{array}$ \\
\hline H01 & $8 / 12-13 / 05$ & Lyman & $12: 11$ & 2.54 & $1376-1755$ \\
\hline H02 & $8 / 13-14 / 05$ & Lyman & $11: 57$ & 1.74 & $1957-2379$ \\
\hline H03 & $8 / 14-15 / 05$ & Lyman & $15: 16$ & 3.36 & $1427-1654$ \\
\hline H04 & $8 / 17 / 05$ & Milne-Edwards & $11: 12$ & 2.61 & $1287-1688$ \\
\hline H05 & $8 / 18 / 05$ & Verrill & $6: 08$ & 1.65 & $1100-1316$ \\
\hline H06 & $8 / 19 / 05$ & Verrill & $16: 06$ & 3.20 & $1511-2111$ \\
\hline H07 & $8 / 20-21 / 05$ & Goode & $13: 02$ & 2.59 & $1859-2134$ \\
\hline H08 & $8 / 22 / 05$ & Kükenthal & $9: 33$ & 4.50 & $818-920$ \\
\hline H09 & $8 / 22-23 / 05$ & Kükenthal & $17: 54$ & 3.58 & $1217-1836$ \\
\hline H10 & $8 / 25 / 05$ & Nashville & $12: 12$ & 1.73 & $2100-2244$ \\
\hline H11 & $8 / 26 / 05$ & Nashville & $13: 14$ & 3.96 & $2232-2561$ \\
\hline H12 & $8 / 27-28 / 05$ & Manning & $16: 53$ & 3.71 & $1322-1330$ \\
\hline H13 & $8 / 29 / 05$ & Rehoboth & $12: 37$ & 1.67 & $1815-1914$ \\
\hline H14 & $8 / 29-30 / 05$ & Rehoboth & $12: 13$ & 2.38 & $1351-1676$ \\
\hline H15 & $8 / 31-9 / 1 / 05$ & Kelvin & $20: 26$ & 4.01 & $1931-2606$ \\
\hline H16 & $9 / 1-2 / 05$ & Balanus & $9: 59$ & 2.10 & $1650-1932$ \\
\hline
\end{tabular}

\section{Taxonomic Composition}

A total of 61,625 observations were made from the video. Many of the fauna have not yet been identified to species and so have been categorized into morphotypes. There were a total of 270 morphotypes observed from the seamounts covering 13 phyla. The most abundant phyla were Porifera (mainly sponges), Cnidaria (mainly corals), and Echinodermata (Figure 9). There were 75 morphotypes that were found only at the Corner Rise seamounts, 60 unique to the New England seamounts, and 135 morphotypes across both regions (Table 2). The phylum Cnidaria was the most abundant fauna in the 
Corner Rise seamounts, followed by the phylum Porifera, while the phylum Porifera dominated on the New England seamounts followed by the phylum Cnidaria. Also, three phyla observed on the Corner Rise were not observed on the Corner Rise seamounts, Hemichordata, Nematoda, and Sipuncula (Figure 10 - Figure 11). General results for each of the phyla encountered are listed below.

\section{Foraminifera}

The foraminiferans observed were xenophyophores observed $\sim 87.9 \%$ of the time on soft sediment. Otherwise they were seen on hard substrate or fossil rubble (Table 2).

\section{Porifera}

There were 28 morphotypes for sponges (Table 2). Many have not yet been identified to species. It was the most abundant group of all phyla and they were found mostly on hard substrate.

\section{Cnidaria}

There were 96 morphotypes within this phylum (Table 2). The most dominant morphotype was the coral Calyptrophora clinata Cairns, 2007, followed by hydroids and the coral Paramuricea biscaya Grasshoff, 1977. The different hydroid species were almost always found on corals, predominantly on skeletons of dead corals. A solitary hydroid was observed two out of four times on hard substrate. Several new species of corals have been described from specimens collected on this cruise: Calyptrophora clinata Cairns 2007 (Cairns 2007), and three new species in the genus Iridogorgia: Iridogorgia fontinalis Watling, 2007, Rhodaniridogorgia fragilis Watling, 2007, and Iridogorgia venusta Watling, 2007 (Watling 2007). 


\section{Ctenophora}

Two morphotypes of ctenophores were observed. Thirteen individuals of ctenophore sp. 1 were observed in the water column while 14 individuals of ctenophore sp. 2 were observed on a sponge (Table 2).

\section{Nematoda}

Only one individual was observed swimming in the water column (Table 2).

\section{Annelida}

Eight morphotypes were observed within this phylum (Table 2). The most abundant were serpulid worms found mainly on hard substrate. Several taxa occurred more than $50 \%$ of the time on a particular substrate. Polychaete $\mathrm{sp} .3$ was found eight times only on the cnidarian Bathypathes aff. lyra Brook, 1889 and Polynoid sp. 2 was observed a total of eight times on three different cnidarian species. A eunicid polychaete was also observed one time on the cnidarian Lophelia sp.

\section{Sipuncula}

Only one individual was observed and recorded (Table 2).

\section{Mollusca}

Eight morphotypes were observed in this phylum (Table 2). The most taxa abundant were gastropods. Egg casings were also observed $\sim 96 \%$ of the time on cnidarians and seen once on a sponge. Several representative eggs were collected along with coral colonies, and subsequent dissection revealed them to be cirrate octopus eggs. 


\section{Arthropoda}

A total of 33 morphospecies were observed within this phylum (Table 2). The most abundant was the barnacle Glyptelasma sp., which was observed a majority of the time on cnidarians, and within cnidarians mainly on coral skeletons. Shrimp sp. 3 was the next most abundant taxon observed mainly on abiotic substrates, such as in the water column, on hard substrate, or soft sediment.

Several taxa occurred more than $50 \%$ of the time on particular substrates. One copepod was observed with a primnoid, and a fish parasite was also observed on a fish, Macrourus aff. Berglax Lacepède, 1801. Two species of shrimp were observed exclusively on corals. Bathypalaemonella serratipalma L. H. Pequegnat, 1970 was observed 29 times on several cnidarians within the family Chrysogorgidae. A potential new species of shrimp within this family, Bathypalaemonella $n$. sp., was also observed two times on the coral Acanella eburnea Pourtalès, 1868.

Numerous galatheid species were observed on a variety of substrates. Most were observed on hard substrate however several species were observed on several cnidarian species. A single individual of Galatheid sp. 2 was observed on a sponge, two individuals of Galatheid sp. 4 was observed on a coral skeleton, and two large individuals of Galatheid sp. 7 was observed on the antipathian coral Plumapathes sp. Uroptychus nitidus Milne-Edwards, 1880 was observed on the coral Acanella eburnea, Uroptychus $s p .1$ was observed twice on an antipatharian (Taxipathes sp. or Sibopathes $s p$.), Uroptychus sp. 2 was observed exclusively on Parantipathes sp. and Uroptychus sp. 3 was observed on Chrysogorgia sp. Uroptychus sp. 1, 2, and 3 are potential new species.

\section{Echinodermata}

There were 58 morphotypes within this phyla dominated by ophiuroids and crinoids (Table 2). Holothurians were found on hard substrate, including fossil coral rubble and soft sediment. The echinoids, asteroids, and brisingids were found mainly on the two substrate types of hard substrate and soft sediment, although a few were observed 
on cnidarians and sponges. The echinoids were observed $\sim 4.3 \%$ of the time on cnidarians and sponges, the asteroids were observed $\sim 7.7 \%$ of the time on cnidarians and sponges, and the brisingids $\sim 28.6 \%$ of the time. The crinoids were observed mostly on corals and sponges, predominantly on coral skeletons and dead sponges. They were also observed on hard substrate. The ophiuroids were found on many substrate types, depending on the species. Asteroschema clavigera Verrill, 1894 was found only on the cnidarians Paragorgia sp. and Paramuricea spp. Ophiocreas oedipus Lyman, 1879 was observed only on the cnidarian Metallogorgia melanotrichos Wright and Studer, 1889. Ophioplinthaca abyssalis Cherbonnier \& Sibouet, 1972 was found on several cnidarian species, but predominantly on the coral Candidella imbricata Johnson, 1862 ( 92\%). In contrast, the ophiuroid Ophioplinthaca chelys Wyville Thomson, 1878 was observed $\sim 79 \%$ of the time on hard substrate or fossil rubble. There is potentially one new species of ophiuroid.

\section{Hemichordata}

Only one morhoptype was observed in this phylum, an acorn worm that was documented while swimming in the water column (Table 2).

\section{Chordata}

There were 40 morphotypes within this phylum; predominantly fish but also salps and tunicates (Table 2). The fish were predominantly found in the water column, although some were observed on the sea floor, such as Bathychaunax spp., Bathypterois spp., Gaidropsarus ensis Reinhardt, 1837, Laemonema goodebeanorum Meléndez C. \& Markle, 1997, and one synamphobranchid eel. The most dominant morphotype identified was Aldrovandia spp, followed by Cyclothone spp. The fish Neobythites gilli Goode \& Bean, 1885 was observed two times (50\%) with the sea urchin, Hygrosoma aff. petersi Agassiz, 1880 and the other two times was found in the water column. 


\section{Substrate Composition}

Six phyla as well as anthropogenic and abiotic sources (hard substrate, soft sediment, and water column) were found to serve as substrate for the organisms observed

in this study (Table 3). Most fauna observed were found on hard substrate throughout the North Atlantic seamounts. The next most abundant substrate on the New England seamounts were the cnidarians followed by the soft sediment and water column (Figure 12). This is different from the substrates observed on the Corner Rise seamounts, where the next most abundant were the soft sediment, water column, and cnidarians (Figure 13).

The hard substrate was inhabited by taxa from the phyla Porifera, Cnidaria, and Echinodermata. The abundant taxa observed on the soft sediment host varied by region. Within the New England Seamount chain, the soft sediment was inhabited most by taxa from the phylum Echinodermata, and then Foraminifera and Cnidaria. On the Corner Rise seamounts, the most abundant taxa found on the soft sediment substrate were cnidarians and then echinoderms and Foraminifera. Within the water column, the most abundant taxa were from the phylum Chordata, mainly fish, followed by Arthropoda, mainly shrimp. The most abundant taxa observed on cnidarians were similar between the New England seamounts and the Corner Rise seamounts. The most abundant taxa were from the phylum Echinodermata, mainly crinoids and ophiuroids. The next most abundant were cnidarians, mainly hydroids, followed by Porifera, mainly sponges.

\section{Host-Associate relationships}

During the cruise, various host-associate relationships were observed among different taxa. In order to identify these relationships and quantify their specificity, percent occurrence of organisms on the various substrates was calculated. Thirty-two species occurred more than $50 \%$ of the time on a particular host substrate. Of these 32 , there were seven species that were observed at least 20 times (Table 4). The hydroid sp. 
2 was observed on coral skeletons $69.5 \%$ of the time; however, it was also found on a variety of other hosts, including hard substrate and animals from different phyla. The ophiuroid Ophioplinthaca abyssalis was observed on a variety of hosts as well, but $91.9 \%$ of all observations were found on the octocoral Candidella imbricata. The ophiuroid Asteroschema clavigera was observed exclusively on two species of octocorals, $66.3 \%$ of the time on Paramuricea sp. and $33.7 \%$ of the time on Paragorgia $s p$. All observations of the ophiuroid Ophiocreas oedipus found it on the octocoral Metallogorgia melanotrichos. It was never observed on any other host type.

Two crustaceans also showed high occurrence on particular substrate hosts. A chirostylid crab, Uroptychus sp. 2, was observed only on antipatharians, $100 \%$ of all observations occurring on Parantipathes sp. The shrimp Bathypalaemonella serratipalma was observed exclusively on three species of Chrysogorgia.

An octopus egg case was observed $72 \%$ of the time on Chrysogorgia sp. but also on several other coral species and coral skeletons.

\section{Multivariate Analyses}

Similarities in faunal assemblages were determined using multivariate statistical analyses. Cluster analysis and MDS indicate that there is a significant difference between the faunal assemblages found on the Corner Rise and New England seamounts. Global 1-way ANOSIM analyses showed increasing significance of this regional difference as sample became more discrete in scale, from sample bins of whole seamounts $(\mathrm{p}=0.016)$ to sample bins of dives $(\mathrm{p}=0.002)$, to sample bins of $100 \mathrm{~m}$ depths within seamounts $(\mathrm{p}=0.001)$ (Figure 14 - Figure 16).

When observations were binned across all seamounts into $100 \mathrm{~m}$ depth bins, cluster analysis and MDS indicated distinct differences between different depth regions of shallow (700-300 m), middle (1300-2300 m), deep (2300-2600 m), and extremely deep (2600-2700 m) which were found to be highly significant by ANOSIM analyses $(\mathrm{p}=0.001)$ (Figure 17 and Figure 18). As the extreme shallow and deep depth ranges 
seemed very different from the rest of the samples, the analysis was repeated excluding the 700-800 $\mathrm{m}$ and 2600-2700 $\mathrm{m}$ depth bins, but the difference between the depth regions of shallow, middle, and deep remained significant $(\mathrm{p}=0.001)$ and the 2D Stress value decreased from 0.1 to 0.09 , indicating greater significance for the clustering pattern of samples seen in the MDS (Figure 18 and Figure 19). SIMPER analysis showed that the difference between the middle depth region of 1300-2300 $\mathrm{m}$ and the deep depth region of 2300-2600 m was accounted for by a change in the abundance of a variety of taxa and the disappearance of a number of taxa (Table 5).

Multivariate statistical analyses were performed with observations grouped as samples of $100 \mathrm{~m}$ depth intervals within each seamount. MDS analyses showed samples clustering by seamount rather than depth in many cases for both the Corner Rise and New England seamounts (Figure 20 and Figure 21). ANOSIM analyses showed this clustering of samples to be significant for both the Corner Rise $(\mathrm{p}=0.001)$ and the New England seamount chain $(\mathrm{p}=0.001)$.

This pattern was observed even more clearly when analyzed using $200 \mathrm{~m}$ depth ranges and including only those samples from seamounts found in those depth regions. MDS analysis for 1500-1700 m samples pattern for all of the seamounts included except for Verrill, where the two samples seemed approximately equally distinct from the other samples (Figure 22). ANOSIM analyses showed this clustering pattern to be significant $(\mathrm{p}=0.002)$. This was also true for all the samples within the $2000-2200 \mathrm{~m}$ depth range, except for the samples from Nashville $(\mathrm{p}=0.003)$ (Figure 23).

Distinct faunal assemblages were also observed through multivariate analysis when observations were grouped by substrate upon which they occurred. Looking at all substrate types, ANOSIM results indicated a significant difference between abiotic and biotic substrates $(\mathrm{p}=0.012)$. The natural abiotic substrates of soft sediment, hard substrate, and open water column are different from the other biotic and abiotic substrate. The dead biotic substrate of the sponges, fossil coral, and coral skeletons lie intermediary between the living biotic substrate and the natural abiotic substrate (Figure 24). 
Within abiotic substrates, ANOSIM analyses indicated a significant difference between natural and anthropogenic abiotic substrate types $(p=0.029)$ with the faunal assemblages on the anthropogenic abiotic substrates being more similar to the faunal assemblages found on living biotic substrates (Figure 24 and Figure 25).

Within biotic substrates, ANOSIM analyses showed a significant difference between the faunal assemblages found on living and dead substrates $(\mathrm{p}=0.001)$ (Figure 26).

As significant host-associate relationships were observed between epifauna on coral hosts, the faunal assemblages found on corals was also analyzed. ANOSIM analyses showed a significant difference between the faunal assemblages found on different coral families ( $\mathrm{p}=0.006$ ) (Figure 27).

The reanalysis of the data with broader taxonomic identifications resulted in similar patterns showing significant differences between faunal assemblages based on geographic region and seamount region. However, the pattern of distinct faunal assemblages seen on different substrates was not seen. 
Table 2. Morphospecies enumerated from the Corner Rise and New England seamounts. They were identified to the lowest taxonomic unit.

\begin{tabular}{|c|c|c|c|c|}
\hline Phylum & Species & $\begin{array}{l}\text { Corner } \\
\text { Rise }\end{array}$ & NESC & Total \\
\hline Foraminifera & $\begin{array}{l}\text { xenophyophore (various } \\
\text { unidentified) }\end{array}$ & 194 & 401 & 595 \\
\hline \multicolumn{5}{|l|}{ Foraminifera } \\
\hline \multirow[t]{3}{*}{ Porifera } & Farrea $s p$. & 189 & 1667 & 1856 \\
\hline & Geodia sp. & 140 & 698 & 838 \\
\hline & $\begin{array}{l}\text { predatory sponge (various } \\
\text { unidentified) }\end{array}$ & 50 & & 50 \\
\hline & Rosselid sp. & 192 & 409 & 601 \\
\hline & sponge (various unidentified) & 7436 & 10084 & 17520 \\
\hline & sponge sp. 1b & & 12 & 12 \\
\hline & sponge sp. 3 & 34 & 15 & 49 \\
\hline & sponge sp. 5 & 68 & 20 & 88 \\
\hline & sponge sp. 5b & 1 & 4 & 5 \\
\hline & sponge sp. 5d & & 122 & 122 \\
\hline & sponge sp. 6 & 5 & 5 & 10 \\
\hline & sponge sp. 6b & 5 & & 5 \\
\hline & sponge sp. 7 & 285 & & 285 \\
\hline & sponge sp. 8 & 1330 & & 1330 \\
\hline & sponge sp. 9 & 5 & & 5 \\
\hline & sponge sp. 10 & 30 & 227 & 257 \\
\hline & sponge sp. 11 & 11 & 2 & 13 \\
\hline & sponge sp. 12 & 1 & 1 & 2 \\
\hline & sponge sp. 13 & 88 & 7 & 95 \\
\hline & sponge sp. 14 & 25 & 2371 & 2396 \\
\hline & sponge sp. 15 & 47 & 4 & 51 \\
\hline & sponge sp. 16 & 8 & & 8 \\
\hline & sponge sp. 17 & 23 & & 23 \\
\hline
\end{tabular}


Table 2. cont,

\begin{tabular}{|c|c|c|c|c|}
\hline Phylum & Species & $\begin{array}{l}\text { Corner } \\
\text { Rise }\end{array}$ & NESC & Total \\
\hline & sponge sp. 19 & 1 & & 1 \\
\hline & sponge sp. 20 & 230 & 2541 & 2771 \\
\hline & sponge sp. 21 & & 21 & 21 \\
\hline & sponge sp. 22 & & 15 & 15 \\
\hline & sponge sp. 23 & 5 & 95 & 100 \\
\hline Porifera Total & & 10209 & 18320 & 28529 \\
\hline Cnidaria* & Acanella sp. & 584 & 1 & 585 \\
\hline & Acanella eburnean & 622 & 81 & 703 \\
\hline & Acanthogorgia sp. & & 2 & 2 \\
\hline & Acanthogorgia aff. granulata & 2 & 1264 & 1266 \\
\hline & anemone (various unidentified) & 52 & 111 & 163 \\
\hline & anemone sp. 1 & 29 & 27 & 56 \\
\hline & anemone sp. 2 & 4 & & 4 \\
\hline & anemone sp. 3 & 39 & 3 & 42 \\
\hline & anemone sp. 4 & 1 & 1 & 2 \\
\hline & anemone sp. 5 & 11 & 26 & 37 \\
\hline & anemone sp. 6 & 15 & 8 & 23 \\
\hline & anemone sp. 7 & 48 & 51 & 99 \\
\hline & anemone sp. $7 \mathrm{~b}$ & 1 & & 1 \\
\hline & anemone sp. 8 & 1 & 4 & 5 \\
\hline & anemone sp. 9 & 2 & & 2 \\
\hline & anemone sp. 10 & 3 & & 3 \\
\hline & anemone sp. 11 & & 6 & 6 \\
\hline & anemone sp.12 & & 5 & 5 \\
\hline & Anthomastus sp. & 6 & 81 & 87 \\
\hline & $\begin{array}{l}\text { Antipatharian (various } \\
\text { unidentified) }\end{array}$ & 2 & 1 & 3 \\
\hline & Bathypathes aff. Iyra & 74 & 336 & 410 \\
\hline & Bathypathes sp. 2 & & 7 & 7 \\
\hline & Calyptrophora clinata & 4370 & & 4370 \\
\hline & Calyptrophora aff. clinata & 4 & 3 & 7 \\
\hline & Calyptrophora microdentata & & 51 & 51 \\
\hline
\end{tabular}


Table 2. cont,

\begin{tabular}{|c|c|c|c|c|}
\hline Phylum & Species & $\begin{array}{c}\text { Corner } \\
\text { Rise }\end{array}$ & NESC & Total \\
\hline & Calyptrophora pillsburyae & & 7 & 7 \\
\hline & Candidella imbricata & 85 & 252 & 337 \\
\hline & Chrysogorgia sp. & 66 & 53 & 119 \\
\hline & Chrysogorgia sp. 1 & 10 & & 10 \\
\hline & Chrysogorgia sp. 2 & & 3 & 3 \\
\hline & Chrysogorgia sp. 3 & 1 & & 1 \\
\hline & coral (various unidentified) & 36 & 52 & 88 \\
\hline & corallium (various unidentified) & 3 & 604 & 607 \\
\hline & Corralium sp. 1 & & 117 & 117 \\
\hline & Corallium sp. 2 & & 128 & 128 \\
\hline & Desmophylum dianthus & 55 & 20 & 75 \\
\hline & Enallopsammia sp. & 35 & 133 & 168 \\
\hline & hydroid (various unidentified) & 2606 & 50 & 2656 \\
\hline & hydroid sp. 1 & & 1 & 1 \\
\hline & hydroid sp. 2 & 808 & 455 & 1263 \\
\hline & hydroid sp. 3 & 47 & & 47 \\
\hline & hydroid sp. 4 & 10 & & 10 \\
\hline & Iridogorgia sp. & 6 & 15 & 21 \\
\hline & Iridogorgia fontinalis & 4 & 58 & 62 \\
\hline & Iridogorgia magnispiralis & 1 & 226 & 227 \\
\hline & Iridogorgia venusta & & 1 & 1 \\
\hline & Isidella sp. & 4 & 121 & 125 \\
\hline & Isidella sp. 1 & & 2 & 2 \\
\hline & jellyfish (various unidentified) & 2 & & 2 \\
\hline & jellyfish sp. 1 & 1 & & 1 \\
\hline & jellyfish sp. 2 & 1 & & 1 \\
\hline & jellyfish sp. 3 & 1 & & 1 \\
\hline & jellyfish sp. 4 & 3 & & 3 \\
\hline & jellyfish sp. 5 & 1 & & 1 \\
\hline & jellyfish sp. 6 & 1 & & 1 \\
\hline & jellyfish sp. 7 & & 1 & 1 \\
\hline & jellyfish sp. 8 & & 1 & 1 \\
\hline
\end{tabular}


Table 2. cont,

\begin{tabular}{|c|c|c|c|c|}
\hline Phylum & Species & $\begin{array}{c}\text { Corner } \\
\text { Rise }\end{array}$ & NESC & Total \\
\hline & Keratoisis sp. & 17 & 112 & 129 \\
\hline & Leiopathes sp. & 2 & 10 & 12 \\
\hline & Lepidisis sp. & 90 & 822 & 912 \\
\hline & Lophelia sp. & 4 & 80 & 84 \\
\hline & Metallogorgia melanotrichos & 32 & 462 & 494 \\
\hline & muriceides pink & 1 & & 1 \\
\hline & Muriceides sp. & & 1 & 1 \\
\hline & Paramuricea biscaya & 1696 & 497 & 2193 \\
\hline & Paragorgia aff. johnsoni & 14 & 411 & 425 \\
\hline & Paramuricea $s p$. & 3 & & 3 \\
\hline & Parantipathes larix & 193 & 89 & 282 \\
\hline & Parantipathes sp. 2 & 5 & 14 & 19 \\
\hline & $\begin{array}{l}\text { pennatulid (various } \\
\text { unidentified) }\end{array}$ & 6 & 9 & 15 \\
\hline & pennatulid sp. 1 & 16 & 8 & 24 \\
\hline & pennatulid sp. 2 & 14 & 51 & 65 \\
\hline & pennatulid sp. $2 b$ & 1 & & 1 \\
\hline & pennatulid sp. 3 & & 132 & 132 \\
\hline & Placagorgia sp. & & 2 & 2 \\
\hline & Plumapathes sp. & 3 & & 3 \\
\hline & primnoid (various unidentified) & & 7 & 7 \\
\hline & Pteridopathes sp. & & 3 & 3 \\
\hline & purple plexaurid & 18 & 66 & 84 \\
\hline & Rhodaniridogorgia aff. Fragilis & & 1 & 1 \\
\hline & Schizopathes sp. & 3 & & 3 \\
\hline & $\begin{array}{l}\text { siphonophore (various } \\
\text { unidentified) }\end{array}$ & 1 & 1 & 2 \\
\hline & solitary hydroid sp. 1 & 2 & 2 & 4 \\
\hline & solitary hydroid sp. 2 & & 1 & 1 \\
\hline & Stauropathes sp. & 9 & 197 & 206 \\
\hline & Stichopathes abyssicola & 9 & & 9 \\
\hline & Stichopathes gracilis & 4 & 11 & 15 \\
\hline
\end{tabular}


Table 2. cont,

\begin{tabular}{|c|c|c|c|c|}
\hline Phylum & Species & $\begin{array}{l}\text { Corner } \\
\text { Rise }\end{array}$ & NESC & Total \\
\hline & Stoloniferous octocoral & & 47 & 47 \\
\hline & Swiftia aff. pallida & 4 & 3 & 7 \\
\hline & Taxipathes sp. & 6 & 5 & 11 \\
\hline & Telesto sp. & 2 & 2 & 4 \\
\hline & Thouarella aff. grasshoffi & 1 & & 1 \\
\hline & Thouarella aff. hilgendorfi & 15 & 10 & 25 \\
\hline & Trachythela rudis & & 54 & 54 \\
\hline & $\begin{array}{l}\text { whip coral (various } \\
\text { unidentified) }\end{array}$ & 3 & 14 & 17 \\
\hline & zoanthid (various unidentified) & 11 & 387 & 398 \\
\hline Cnidaria Total & & 11842 & 7879 & 19721 \\
\hline Ctenophora & $\begin{array}{l}\text { ctenophore (various } \\
\text { unidentified) }\end{array}$ & 9 & 4 & 13 \\
\hline & ctenophore sp. 2 & 14 & & 14 \\
\hline $\begin{array}{l}\text { Ctenophora } \\
\text { Total }\end{array}$ & & 23 & 4 & 27 \\
\hline Nematoda & nematode & 1 & & 1 \\
\hline Nematoda Total & & 1 & & 1 \\
\hline Annelida & Aphrodita sp. & & 2 & 2 \\
\hline & echiuran (various unidentified) & 1 & 2 & 3 \\
\hline & eunicid polychaete & & 1 & 1 \\
\hline & $\begin{array}{l}\text { polychaete (various } \\
\text { unidentified) }\end{array}$ & 9 & 14 & 23 \\
\hline & polychaete sp. 3 & 3 & 5 & 8 \\
\hline & polynoid (various unidentified) & 1 & & 1 \\
\hline & polynoid sp. 2 & 5 & 3 & 8 \\
\hline & $\begin{array}{l}\text { serpulid worm (various } \\
\text { unidentified) }\end{array}$ & 38 & 1 & 39 \\
\hline Annelida Total & & 57 & 28 & 85 \\
\hline Sipuncula & sipunculan worm & 1 & & 1 \\
\hline Sipuncula Total & & 1 & & 1 \\
\hline Mollusca & aplacaphora (various & 10 & 8 & 18 \\
\hline
\end{tabular}


Table 2. cont,

\begin{tabular}{|c|c|c|c|c|}
\hline Phylum & Species & $\begin{array}{c}\text { Corner } \\
\text { Rise }\end{array}$ & NESC & Total \\
\hline \multicolumn{5}{|c|}{ unidentified) } \\
\hline & chiton (various unidentified) & & 4 & 4 \\
\hline & $\begin{array}{l}\text { cirrate octopus egg (various } \\
\text { unidentified) }\end{array}$ & 17 & 9 & 26 \\
\hline & clam & 1 & & 1 \\
\hline & $\begin{array}{l}\text { gastropod (various } \\
\text { unidentified) }\end{array}$ & 10 & 17 & 27 \\
\hline & gastropod sp. 1 & 1 & & 1 \\
\hline & gastropod sp. 2 & 1 & & 1 \\
\hline & squid & 1 & & 1 \\
\hline Mollusca Total & & 41 & 38 & 79 \\
\hline Arthropoda* & $\begin{array}{l}\text { amphipod (various } \\
\text { unidentified) }\end{array}$ & 15 & 13 & 28 \\
\hline & barnacle (various unidentified) & & 11 & 11 \\
\hline & barnacle sp. 3 & & 9 & 9 \\
\hline & Bathypalaemonella n.sp. & 1 & 2 & 3 \\
\hline & $\begin{array}{l}\text { Bathypalaemonella } \\
\text { serratipalma }\end{array}$ & 19 & 10 & 29 \\
\hline & copepod (various unidentified) & & 1 & 1 \\
\hline & crab (various unidentified) & 22 & 16 & 38 \\
\hline & crab sp. 1 & 17 & 1 & 18 \\
\hline & crab sp. 2 & 17 & & 17 \\
\hline & crab sp. 3 & 1 & & 1 \\
\hline & cumacean & & 1 & 1 \\
\hline & galatheid sp. 1 & 48 & 75 & 123 \\
\hline & galatheid sp. 2 & & 1 & 1 \\
\hline & galatheid sp. 4 & 2 & & 2 \\
\hline & galatheid sp. 6 & 1 & & 1 \\
\hline & galatheid sp. 7 & 2 & & 2 \\
\hline & glyptelasma sp. & 203 & 358 & 561 \\
\hline & $\begin{array}{l}\text { hermit crab (various } \\
\text { unidentified) }\end{array}$ & 41 & 33 & 74 \\
\hline
\end{tabular}


Table 2. cont,

\begin{tabular}{|c|c|c|c|c|}
\hline Phylum & Species & $\begin{array}{c}\text { Corner } \\
\text { Rise }\end{array}$ & NESC & Total \\
\hline & isopod (various unidentified) & & 4 & 4 \\
\hline & mysid (various unidentified) & 8 & 4 & 12 \\
\hline & parasitic amphipod & 1 & & 1 \\
\hline & parasitic isopod? & 1 & & 1 \\
\hline & $\begin{array}{l}\text { pycnogonid (various } \\
\text { unidentified) }\end{array}$ & 4 & 5 & 9 \\
\hline & pycnogonid sp. 1 & 3 & & 3 \\
\hline & pycnogonid sp. 2 & 1 & 1 & 2 \\
\hline & pycnogonid sp. 3 & 3 & & 3 \\
\hline & shrimp (various unidentified) & 59 & 22 & 81 \\
\hline & shrimp sp. 1 & 1 & & 1 \\
\hline & shrimp sp. 3 & 198 & 143 & 341 \\
\hline & shrimp sp. 4 & 22 & & 22 \\
\hline & shrimp sp. 8 & 1 & & 1 \\
\hline & shrimp sp. 9 & 1 & & 1 \\
\hline & Uroptychus nitidus & 16 & & 16 \\
\hline & Uroptychus sp. 1 & 2 & & 2 \\
\hline & Uroptychus sp. 2 & 39 & 35 & 74 \\
\hline & Uroptychus sp. 3 & 1 & 4 & 5 \\
\hline $\begin{array}{l}\text { Arthropoda } \\
\text { Total }\end{array}$ & & 750 & 749 & 1499 \\
\hline Echinodermata* & asteroid (various unidentified) & 85 & 121 & 206 \\
\hline & asteroid sp. 5 & 6 & 3 & 9 \\
\hline & asteroid sp. 6 & 7 & 12 & 19 \\
\hline & asteroid sp. 7 & 63 & & 63 \\
\hline & asteroid sp. 8 & & 1 & 1 \\
\hline & asteroid sp. 9 & 2 & & 2 \\
\hline & asteroid sp. 10 & & 10 & 10 \\
\hline & asteroid sp. 11 & & 2 & 2 \\
\hline & Asteroschema clavigera & 45 & 681 & 726 \\
\hline & Astrobranchion sp. & & 1 & 1 \\
\hline & Astrochele lymani & 8 & 8 & 16 \\
\hline
\end{tabular}


Table 2. cont,

\begin{tabular}{|c|c|c|c|c|}
\hline Phylum & Species & $\begin{array}{c}\text { Corner } \\
\text { Rise }\end{array}$ & NESC & Total \\
\hline & Astroporpa sp. & & 9 & 9 \\
\hline & brisingid (various unidentified) & 5 & 93 & 98 \\
\hline & brisingid sp. 1 & 6 & & 6 \\
\hline & brisingid sp. 2 & & 9 & 9 \\
\hline & brisingid sp. 3 & & 27 & 27 \\
\hline & crinoid (various unidentified) & 166 & 188 & 354 \\
\hline & crinoid sp. 1 & 49 & & 49 \\
\hline & crinoid sp. 2 & 583 & 434 & 1017 \\
\hline & crinoid sp. 3 & 666 & 48 & 714 \\
\hline & crinoid sp. 4 & 66 & 61 & 127 \\
\hline & crinoid sp. 5 & 151 & 265 & 416 \\
\hline & crinoid sp. 5b & 40 & & 40 \\
\hline & crinoid sp. 5c & 10 & 1 & 11 \\
\hline & crinoid sp. 6 & 42 & & 42 \\
\hline & crinoid sp. 7 & 32 & 167 & 199 \\
\hline & crinoid sp. 8 & 34 & & 34 \\
\hline & crinoid sp. 9 & 1 & & 1 \\
\hline & $\begin{array}{l}\text { holothurian (various } \\
\text { unidentified) }\end{array}$ & 7 & 5 & 12 \\
\hline & holothurian sp. 1 & 41 & 38 & 79 \\
\hline & holothurian sp. 2 & 3 & 1 & 4 \\
\hline & holothurian sp. 3 & 4 & 2 & 6 \\
\hline & holothurian sp. 5 & 18 & 2 & 20 \\
\hline & holothurian sp. 6 & 2 & & 2 \\
\hline & holothurian sp. 7 & 1 & 32 & 33 \\
\hline & holothurian sp. 8 & & 265 & 265 \\
\hline & holothurian sp. 9 & & 5 & 5 \\
\hline & holothurian sp. 10 & & 33 & 33 \\
\hline & holothurian sp. 11 & & 2 & 2 \\
\hline & holothurian sp. 12 & & 5 & 5 \\
\hline & holothurian sp. 14 & & 7 & 7 \\
\hline & Hygrosoma aff. petersi & 201 & 26 & 227 \\
\hline
\end{tabular}


Table 2. cont,

\begin{tabular}{|c|c|c|c|c|}
\hline Phylum & Species & $\begin{array}{l}\text { Corner } \\
\text { Rise }\end{array}$ & NESC & Total \\
\hline & $\begin{array}{l}\text { ophiacanthid (various } \\
\text { unidentified) }\end{array}$ & 4 & 186 & 190 \\
\hline & Ophiocreas oedipus & 30 & 450 & 480 \\
\hline & Ophiocten centobi & & 286 & 286 \\
\hline & Ophiolinthaca abyssalis & 123 & 1162 & 1285 \\
\hline & Ophiomusium Iymani & 14 & 34 & 48 \\
\hline & Ophioplinthaca chelys & 365 & 876 & 1241 \\
\hline & ophiuroid (various unidentified) & 139 & 660 & 799 \\
\hline & Phormosoma sp. & 40 & 34 & 74 \\
\hline & $\begin{array}{l}\text { sea urchin (various } \\
\text { unidentified) }\end{array}$ & 5 & 36 & 41 \\
\hline & sea urchin sp. 1 & & 1 & 1 \\
\hline & sea urchin sp. 5 & 42 & & 42 \\
\hline & sea urchin sp. 6 & 12 & & 12 \\
\hline & sea urchin sp. 7 & 33 & 20 & 53 \\
\hline & sea urchin sp. 8 & 1 & 2 & 3 \\
\hline & sea urchin sp. 9 & & 401 & 401 \\
\hline & sea urchin sp. 10 & & 1 & 1 \\
\hline $\begin{array}{l}\text { Echinodermata } \\
\text { Total }\end{array}$ & & 3152 & 6713 & 9865 \\
\hline Hemichordata & acorn worm & 1 & & 1 \\
\hline $\begin{array}{l}\text { Hemichordata } \\
\text { Total }\end{array}$ & & 1 & & 1 \\
\hline Chordata* & Aldrovandia spp. & 151 & 60 & 211 \\
\hline & Antimora rostrata & 8 & 9 & 17 \\
\hline & Aphyonidae & & 1 & 1 \\
\hline & Apristurus sp. & 8 & 1 & 9 \\
\hline & Bathiidae & & 1 & 1 \\
\hline & Bathychaunax Roseus & 1 & & 1 \\
\hline & Bathychaunax aff. sp. & 3 & 8 & 11 \\
\hline & Bathygadus spp. & 2 & & 2 \\
\hline & Bathygadus sp. 1 & 2 & 3 & 5 \\
\hline
\end{tabular}


Table 2. cont,

\begin{tabular}{|c|c|c|c|c|}
\hline Phylum & Species & $\begin{array}{l}\text { Corner } \\
\text { Rise }\end{array}$ & NESC & Total \\
\hline & Bathygadus sp. 2 & 2 & & 2 \\
\hline & Bathymicrops Regis & 2 & & 2 \\
\hline & Bathypterois phenax & 4 & & 4 \\
\hline & Bathypterois sp. & 6 & 2 & 8 \\
\hline & Bathysaurus ferox & & 1 & 1 \\
\hline & Boryphaenoides rupestris & & 11 & 11 \\
\hline & Cyclothone sp. & 88 & 59 & 147 \\
\hline & Eurypharynx pelecanoides & 1 & 2 & 3 \\
\hline & unidentified fish & & 1 & 1 \\
\hline & fish (various unidentified) & 466 & 84 & 550 \\
\hline & fish eggs (various unidentified) & 4 & 1 & 5 \\
\hline & Gaidropsarus ensis & 1 & & 1 \\
\hline & Gaidropsus ensis & 1 & 2 & 3 \\
\hline & Halosauropsis macrochir & 5 & 2 & 7 \\
\hline & Hoplostethus mediterraneus & 30 & & 30 \\
\hline & Hoplunnis tenuis & & 2 & 2 \\
\hline & Hydrolagus pallidus & 1 & 1 & 2 \\
\hline & Laemonema goodebeanorum & 7 & & 7 \\
\hline & Lepidion sp. & 4 & & 4 \\
\hline & Luciobrotula corethromycter & 1 & & 1 \\
\hline & Macourus aff. berglax & 33 & 16 & 49 \\
\hline & Neobythites gilli & 4 & & 4 \\
\hline & Neocyttus helgae & 31 & 26 & 57 \\
\hline & $\begin{array}{l}\text { neoscopeliid (various } \\
\text { unidentified) }\end{array}$ & 3 & & 3 \\
\hline & Physiculus sp. & 1 & & 1 \\
\hline & pyrosome & 1 & & 1 \\
\hline & Rouleina sp. & 4 & & 4 \\
\hline & salp (various unidentified) & 4 & & 4 \\
\hline & $\begin{array}{l}\text { synamphobranchid eel } \\
\text { (various unidentified) }\end{array}$ & 11 & & 11 \\
\hline & tunicate (various unidentified) & 8 & 21 & 29 \\
\hline
\end{tabular}


Table 2. cont,

\begin{tabular}{|l|l|c|c|c|}
\hline Phylum & & Corner & & \\
& Species & Rise & NESC & Total \\
\hline \multicolumn{2}{|c|}{ Venefica procera } & 7 & & 7 \\
\hline Chordata Total & 2 & & 2 \\
\hline Total & 27178 & 34447 & 61625 \\
\hline
\end{tabular}

*Morphospecies determination by J. Adkins (Cnidaria), S. Cairns (Cnidaria), S. France (Cnidaria),

R. Waller (Cnidaria), L. Watling (Cnidaria), M. Nizinski (Arthropoda), K. Schnabel (Arthropoda), C. Ahearn (Echinodermata), W. Cho (Echinodermata), P. Auster (Chordata), J. Moore (Chordata)

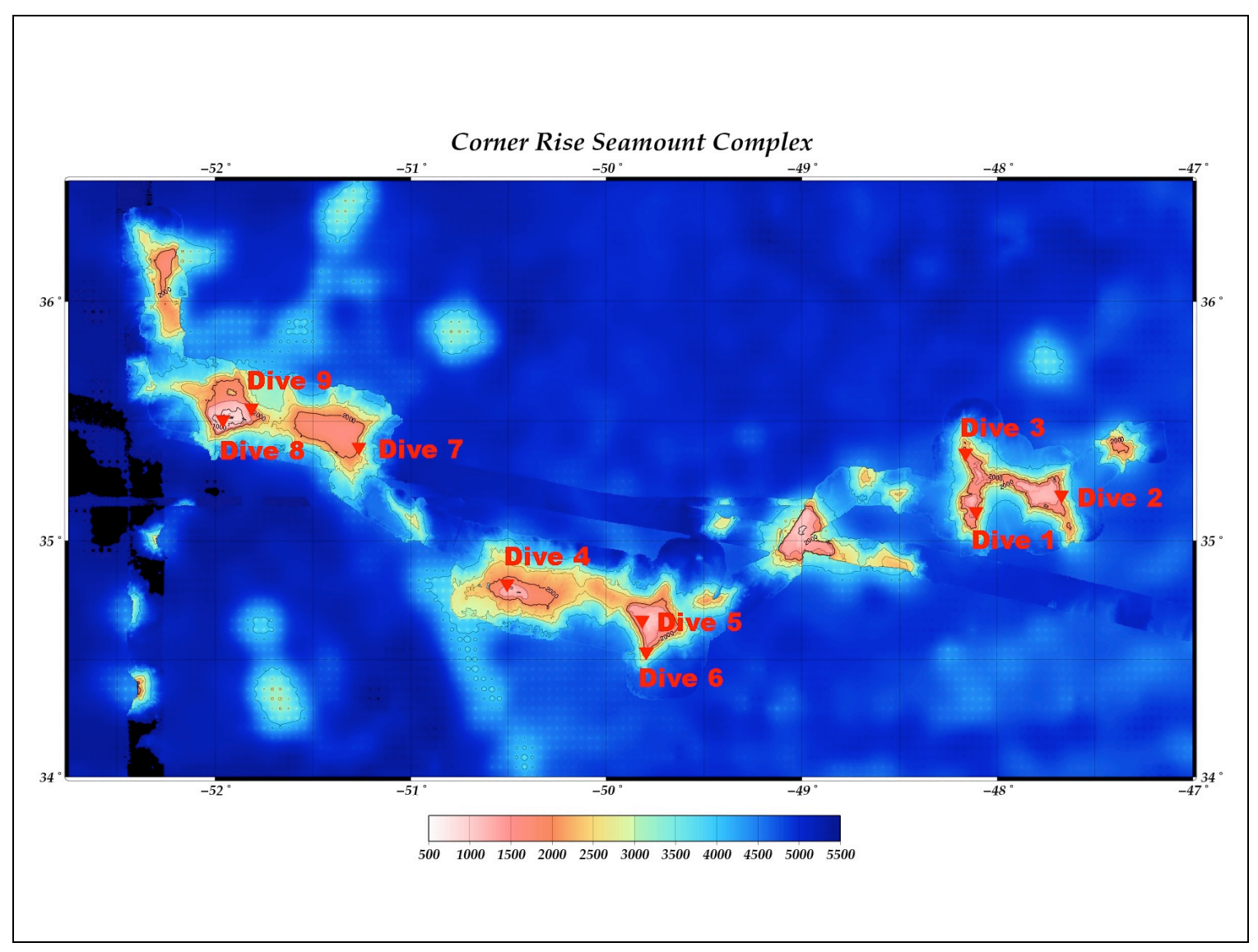

Figure 3. Map of dive sites on peaks of the Corner Rise seamounts created from multibeam bathymetric data collected using a SeaBeam 2120, $20 \mathrm{kHz}$ system with 151 beams in a 150 degree fan-shaped array (Gontz et al. in prep/press). 


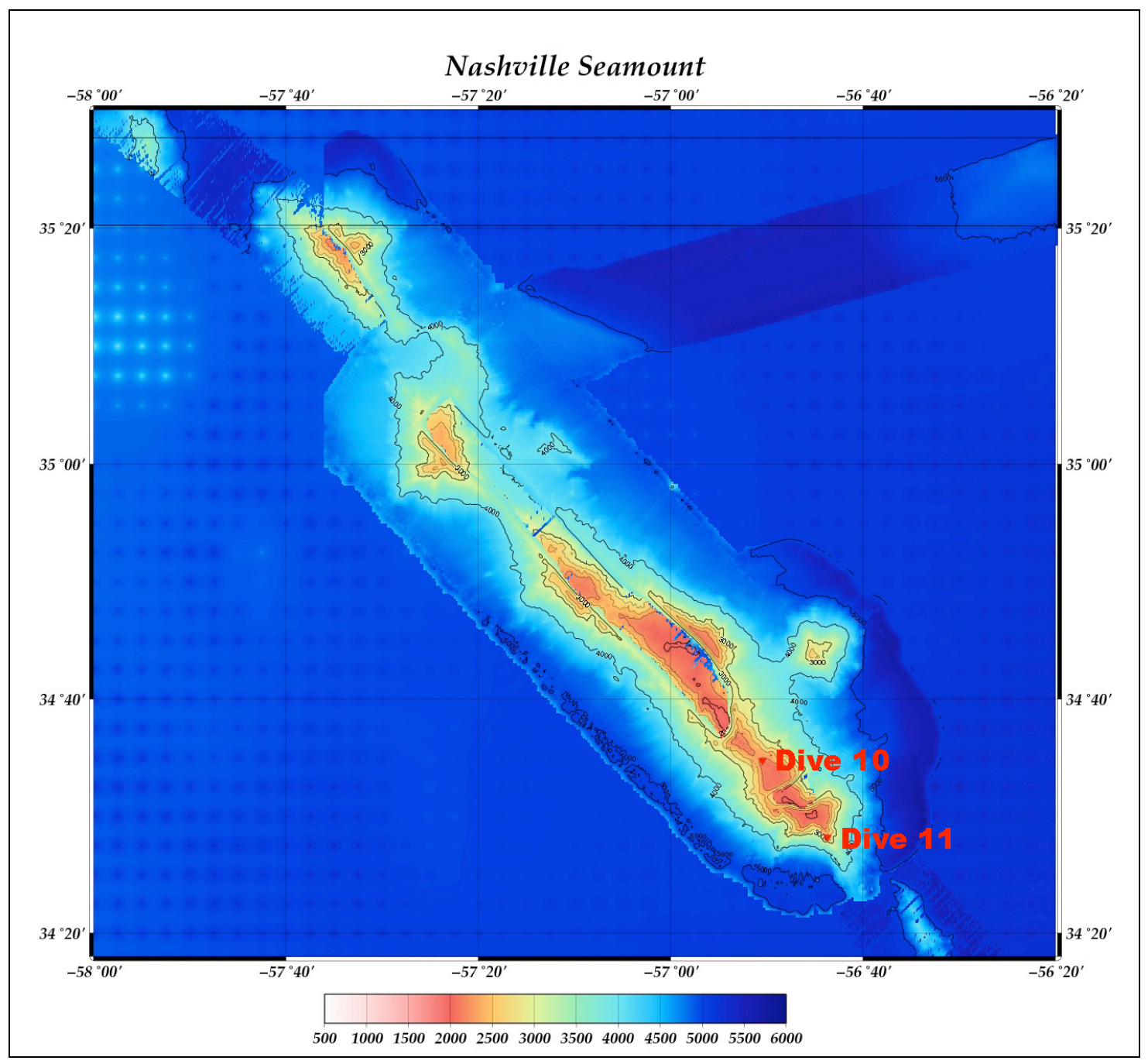

Figure 4. Map of dive sites on Nashville seamount created from multibeam bathymetric data collected using a SeaBeam $2120,20 \mathrm{kHz}$ system with 151 beams in a 150 degree fan-shaped array (Gontz et al. in prep/press). 


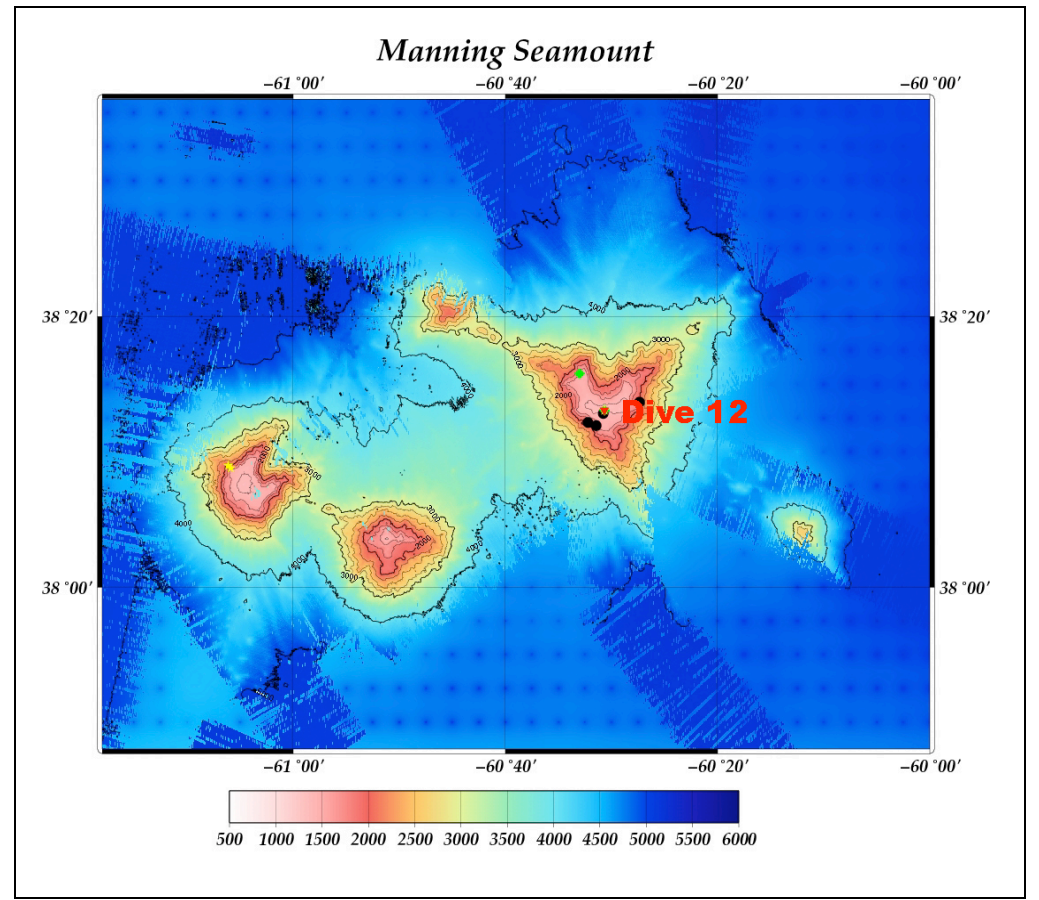

Figure 5. Map of dive sites on Manning seamount created from multibeam bathymetric data collected using a SeaBeam $2120,20 \mathrm{kHz}$ system with 151 beams in a 150 degree fan-shaped array (Gontz et al. in prep/press). 


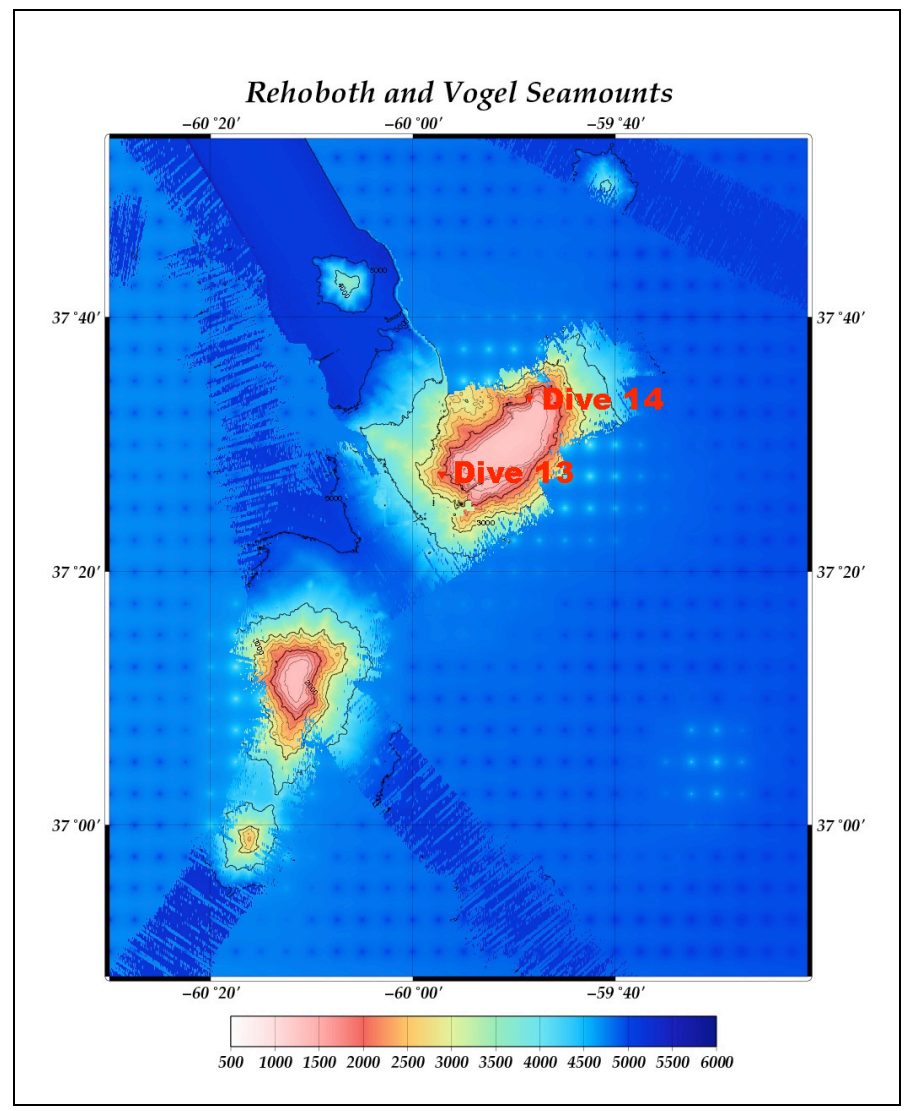

Figure 6. Map of dive sites on Rehoboth seamount created from multibeam bathymetric data collected using a SeaBeam 2120, $20 \mathrm{kHz}$ system with 151 beams in a 150 degree fan-shaped array (Gontz et al. in prep/press). 


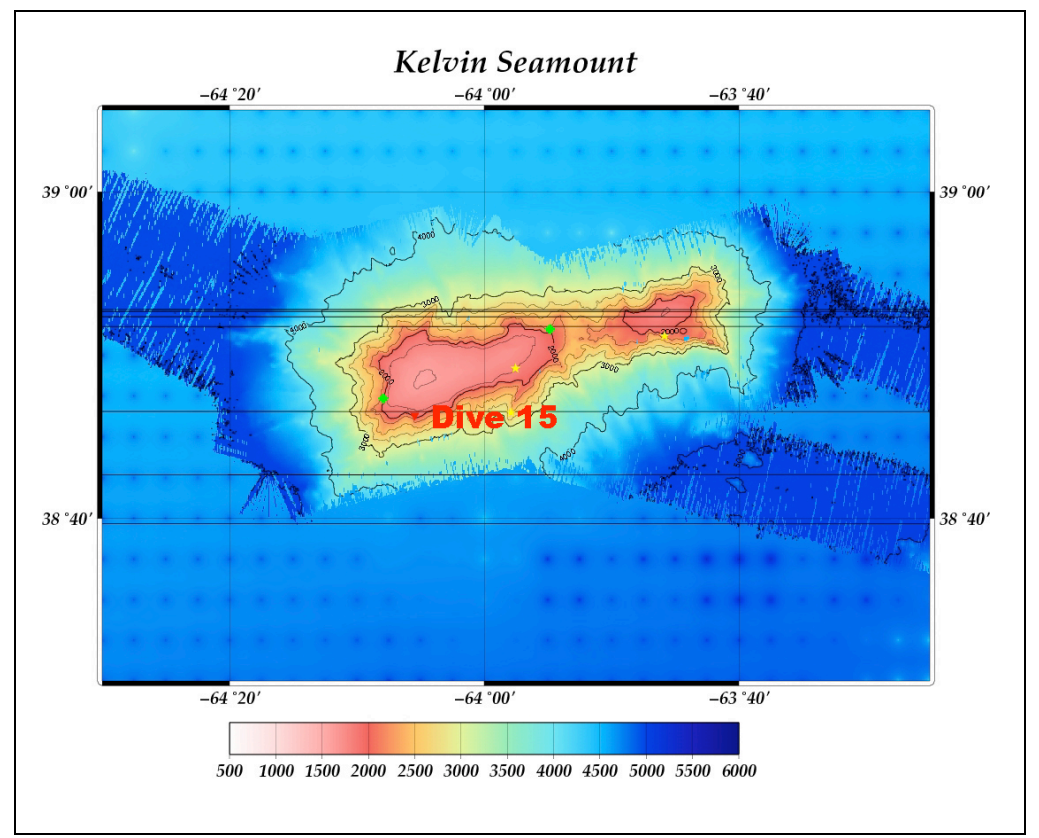

Figure 7. Map of dive sites on Kelvin seamount created from multibeam bathymetric data collected using a SeaBeam $2120,20 \mathrm{kHz}$ system with 151 beams in a 150 degree fan-shaped array (Gontz et al. in prep/press). 


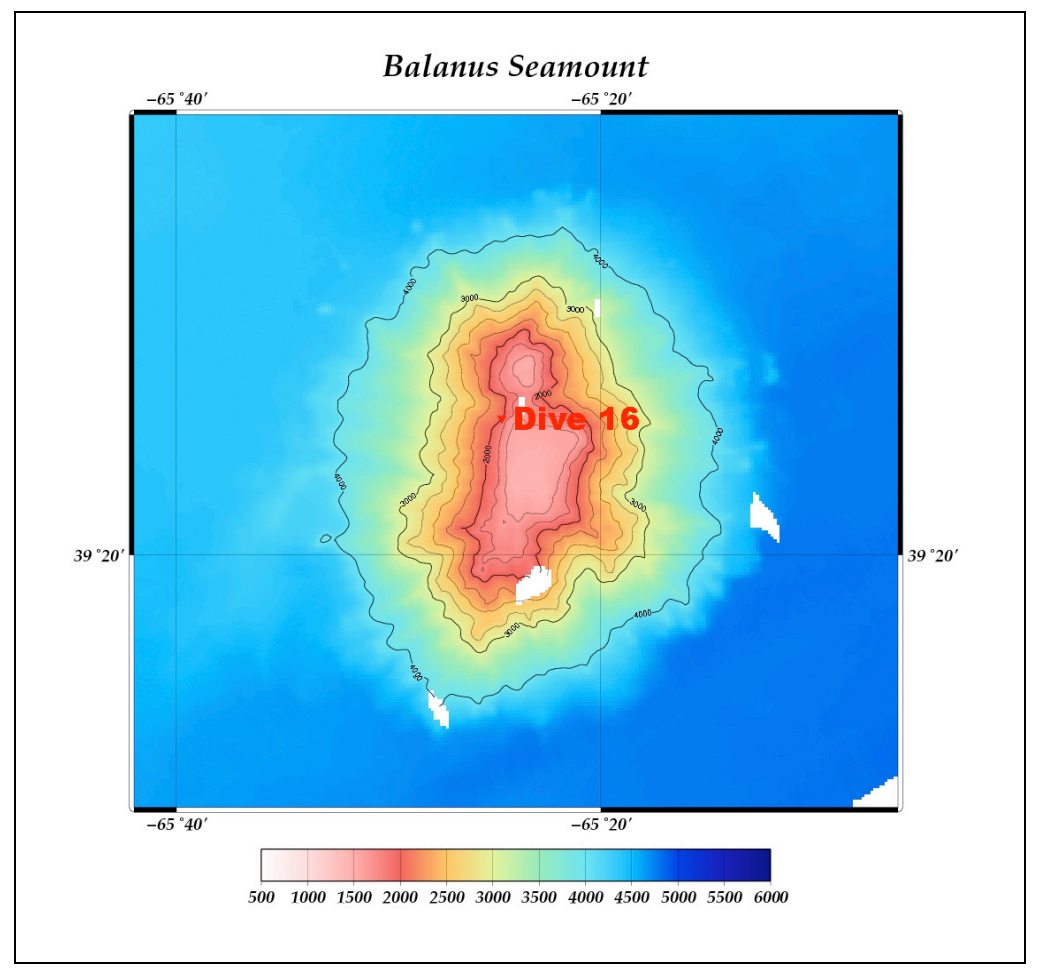

Figure 8. Map of dive sites on Balanus seamount created from multibeam bathymetric data collected using a SeaBeam $2120,20 \mathrm{kHz}$ system with 151 beams in a 150 degree fan-shaped array (Gontz et al. in prep/press). 


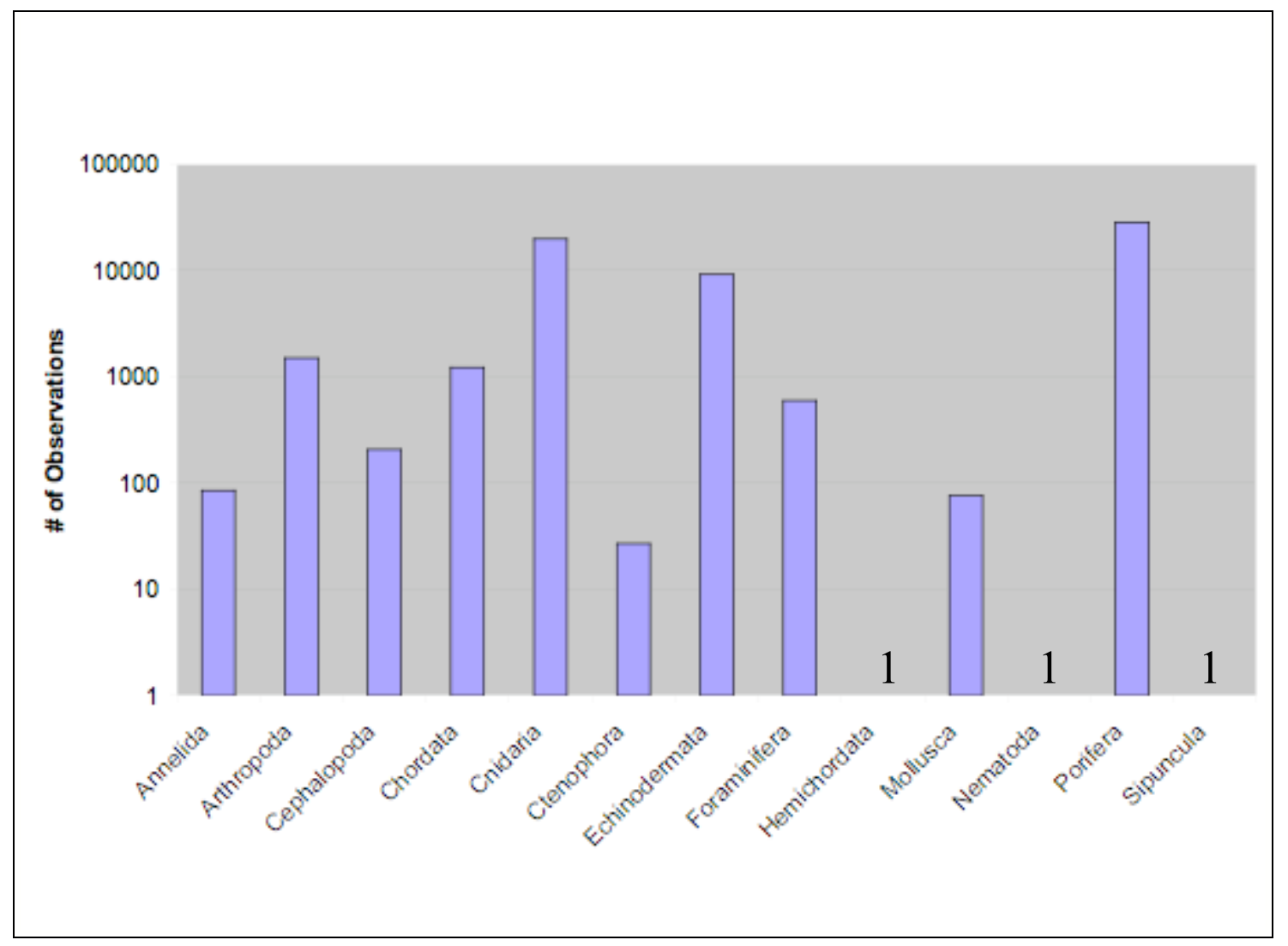

Figure 9. Observations of fauna on the New England and Corner Rise seamounts organized by phyla (logarithmic scale). 


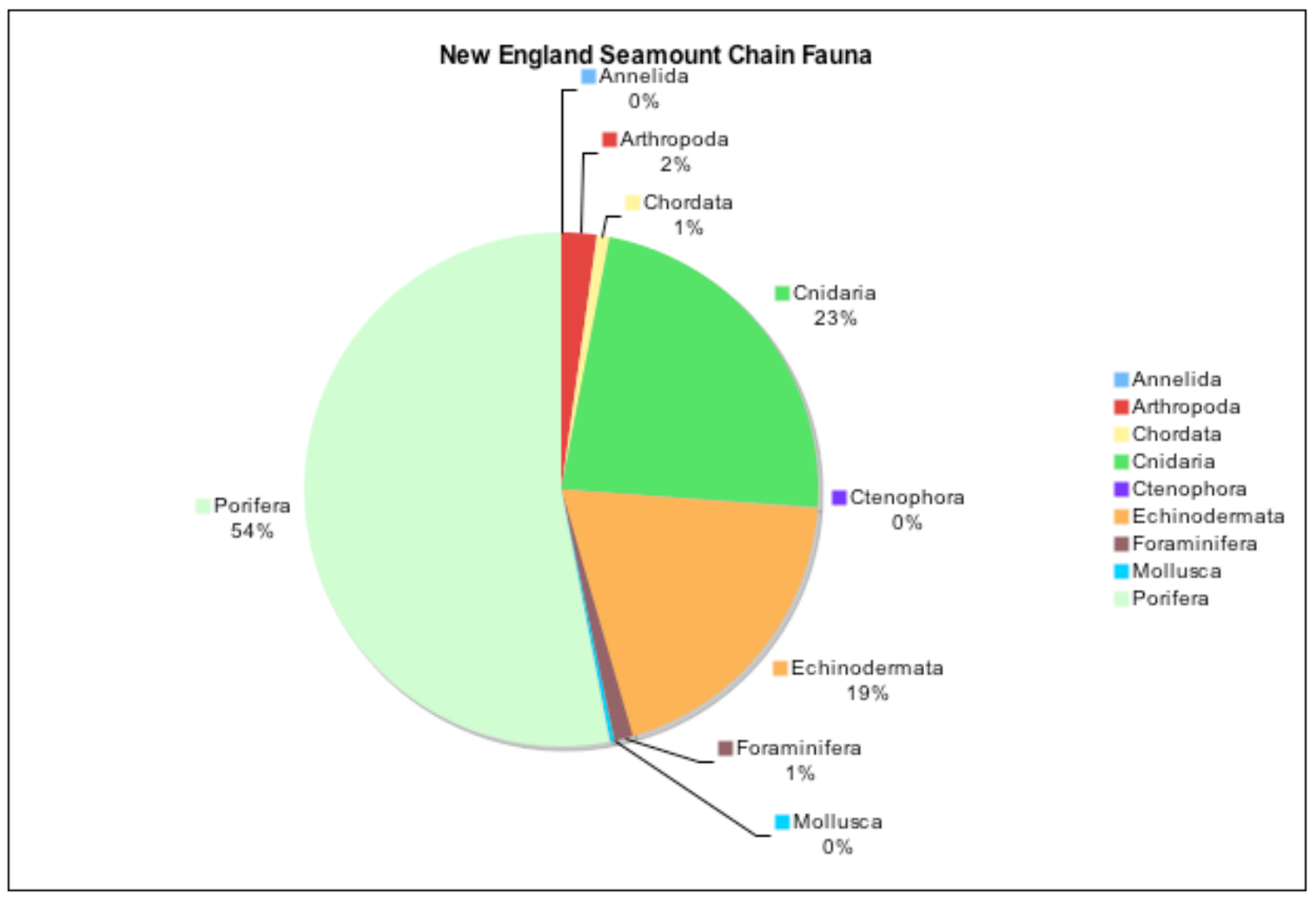

Figure 10. Taxonomic composition by phyla of fauna on the New England seamount chain. 


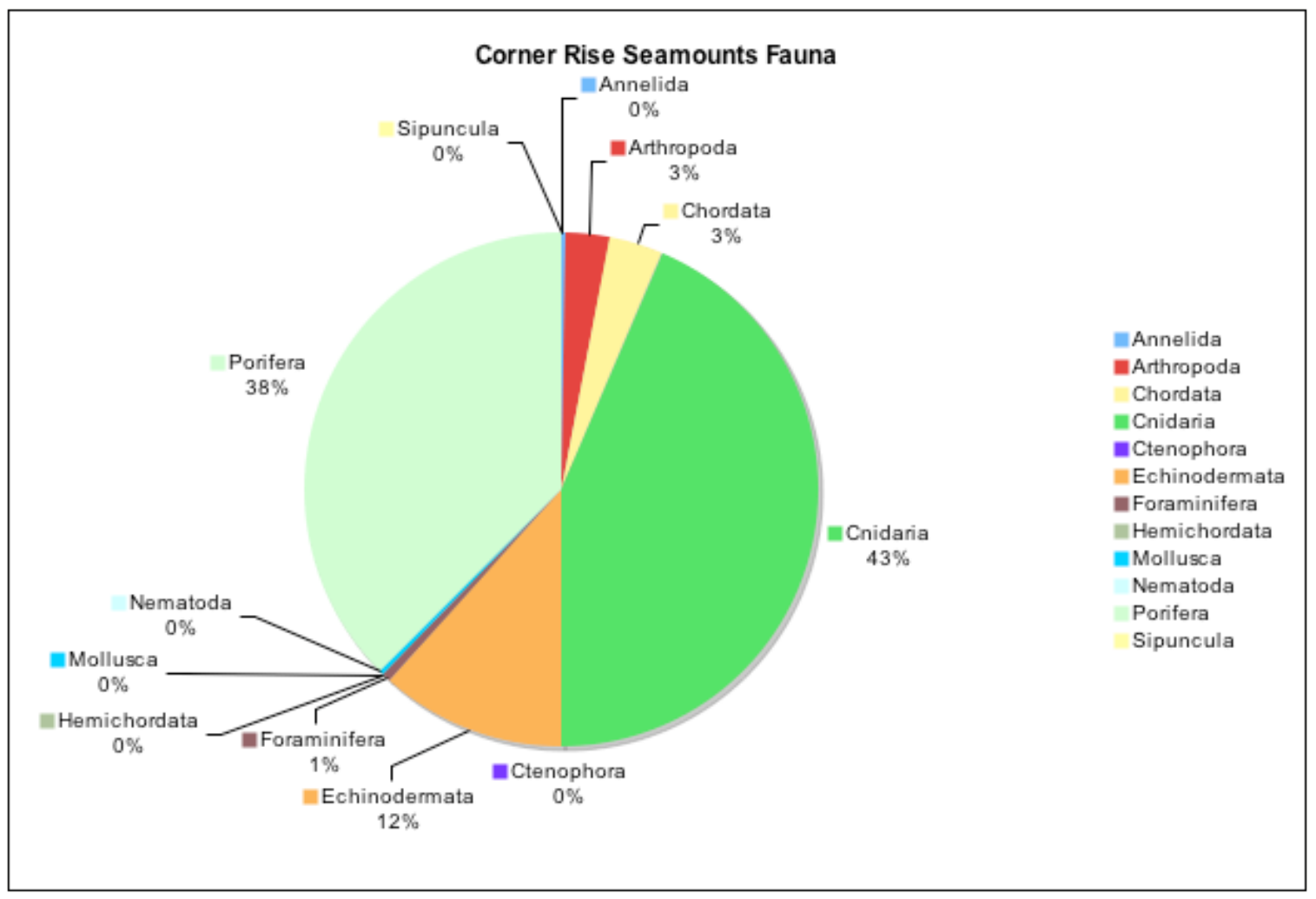

Figure 11. Taxonomic composition by phyla of fauna on the Corner Rise seamounts. 


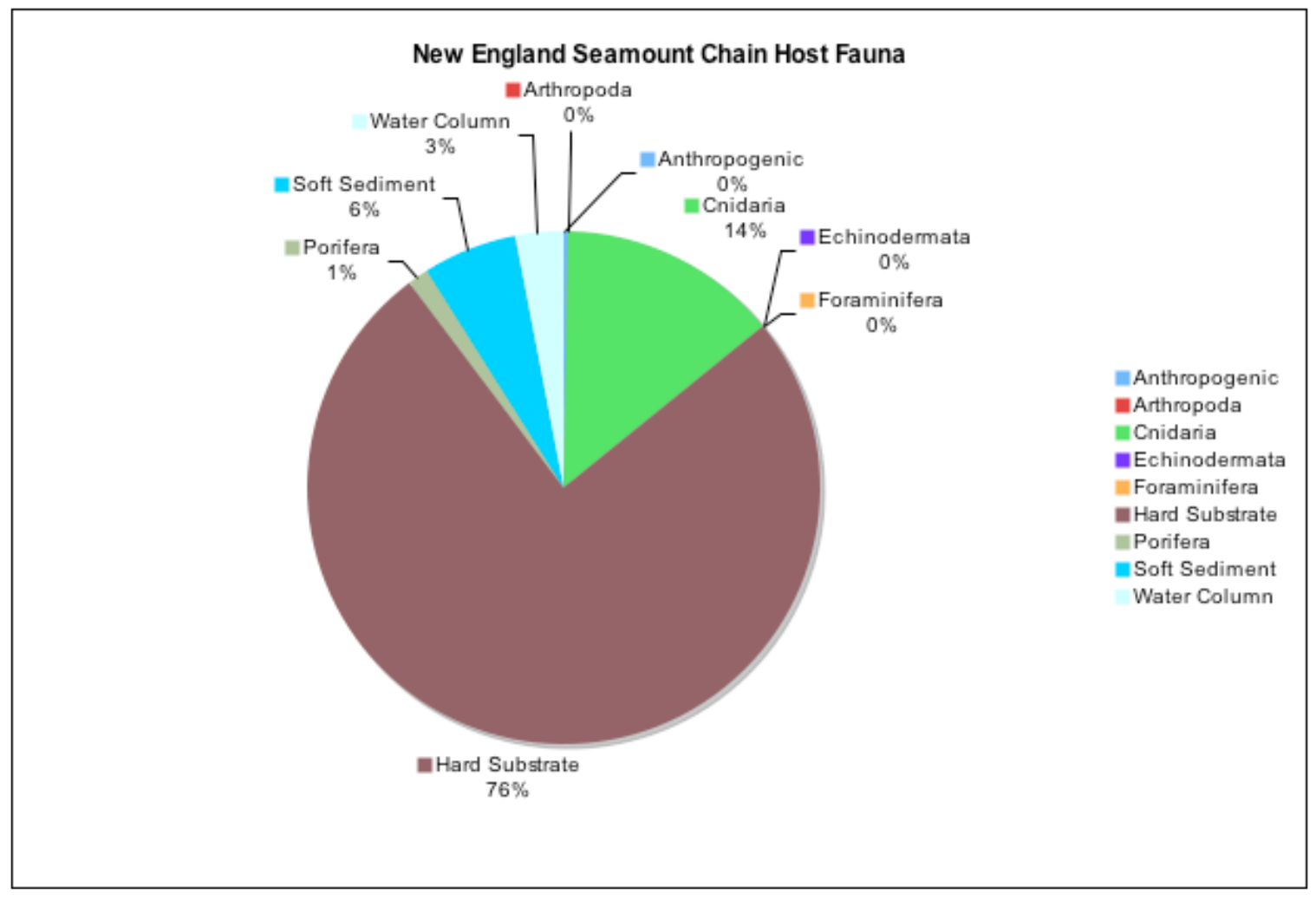

Figure 12. Composition of Hosts by Phyla observed on the New England seamounts. 


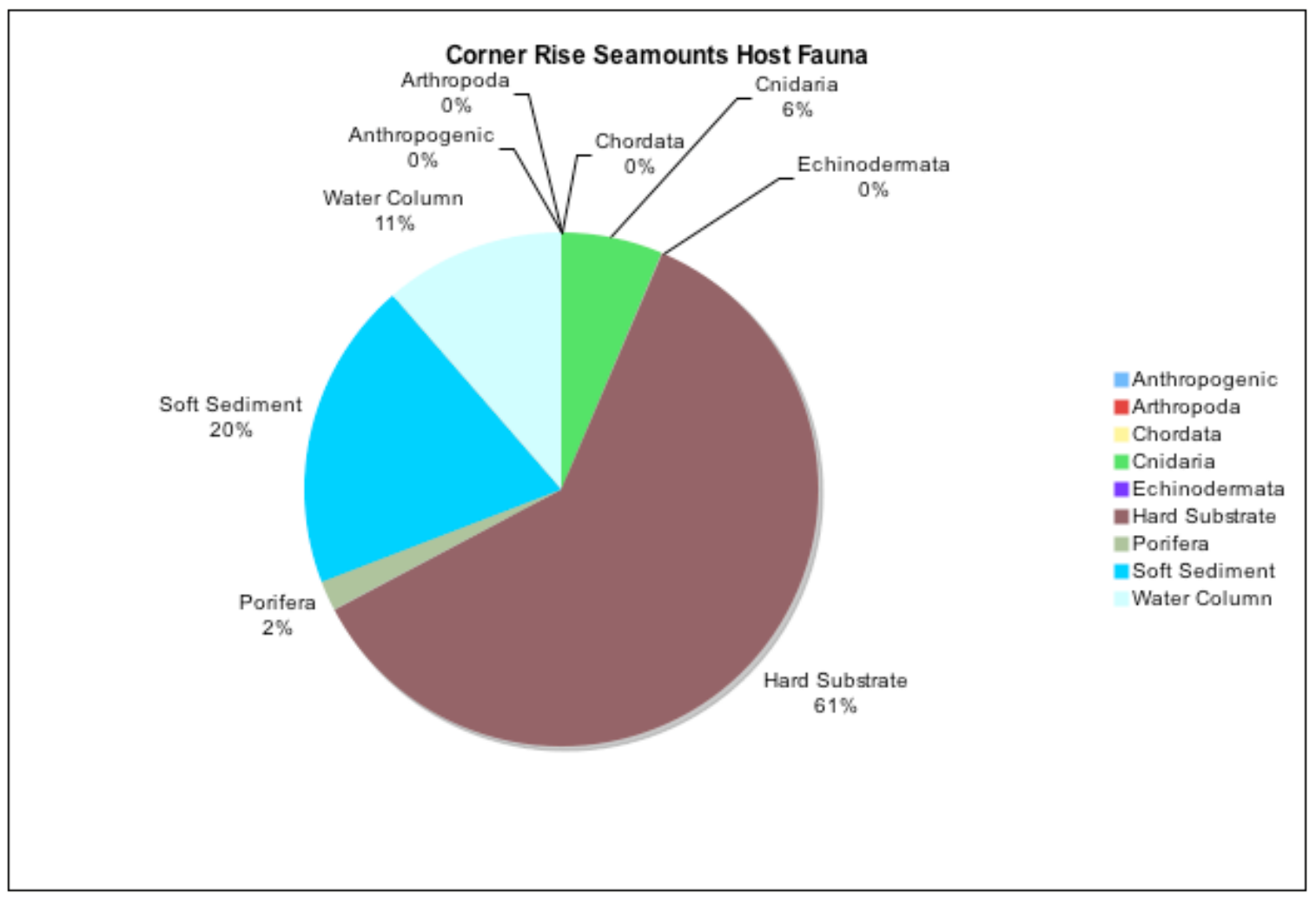

Figure 13. Composition of Hosts by Phyla observed on the Corner Rise seamounts. 
Table 3. Table of Hosts observed on the Corner Rise and New England seamounts.

\begin{tabular}{|c|c|c|c|c|}
\hline Host Phylum & Host Species & $\begin{array}{l}\text { Corner } \\
\text { Rise }\end{array}$ & NESC & Total \\
\hline Foraminifera & xenophyophore & & 2 & 2 \\
\hline \multicolumn{2}{|c|}{ Foraminifera Total } & & 2 & 2 \\
\hline \multirow[t]{2}{*}{ Porifera } & $\begin{array}{l}\text { dead_sponge } \\
\text { Farrea sp. } \\
\text { Geodia sp. } \\
\text { Rosselid sp. } \\
\text { sponge } \\
\text { sponge sp. } 3 \\
\text { sponge sp. } 5\end{array}$ & $\begin{array}{c}50 \\
10 \\
2 \\
12 \\
33 \\
2 \\
2\end{array}$ & $\begin{array}{c}48 \\
16 \\
3 \\
4 \\
39\end{array}$ & $\begin{array}{c}98 \\
26 \\
5 \\
16 \\
72 \\
2 \\
2\end{array}$ \\
\hline & $\begin{array}{l}\text { sponge sp. } 10 \\
\text { sponge sp. } 11 \\
\text { sponge sp. } 12 \\
\text { sponge sp. } 13 \\
\text { sponge sp. } 15 \\
\text { sponge sp. } 16 \\
\text { sponge sp. } 20\end{array}$ & $\begin{array}{l}6 \\
2 \\
12 \\
14 \\
2 \\
9\end{array}$ & $\begin{array}{c}32 \\
1\end{array}$ & $\begin{array}{c}38 \\
2 \\
1 \\
12 \\
14 \\
2 \\
56\end{array}$ \\
\hline \multicolumn{2}{|l|}{ Porifera Total } & 156 & 190 & 346 \\
\hline \multirow[t]{9}{*}{ Cnidaria } & $\begin{array}{l}\text { Acanella eburnea } \\
\text { Acanthogorgia aff. granulata } \\
\text { Bathypathes aff. lyra } \\
\text { Calyptrophora microdentata } \\
\text { Calyptrophora pillsburyae }\end{array}$ & 25 & $\begin{array}{c}14 \\
44 \\
5 \\
8 \\
6\end{array}$ & $\begin{array}{c}39 \\
44 \\
9 \\
8 \\
6\end{array}$ \\
\hline & Candidella imbricata & 30 & 110 & 140 \\
\hline & Chrysogorgia & 35 & 17 & 52 \\
\hline & Chrysogorgia sp. 1 & 12 & & 12 \\
\hline & Chrysogorgia sp. 2 & & 3 & 3 \\
\hline & $\begin{array}{l}\text { Chrysogorgia sp. } 3 \\
\text { coral (various unidentified) }\end{array}$ & 1 & 1 & $\begin{array}{l}1 \\
1\end{array}$ \\
\hline & Corallium spp. & 1 & 35 & 36 \\
\hline & Corralium sp. 1 & & 7 & 7 \\
\hline & Corallium sp. 2 & & 2 & 2 \\
\hline
\end{tabular}


Table 3. cont,

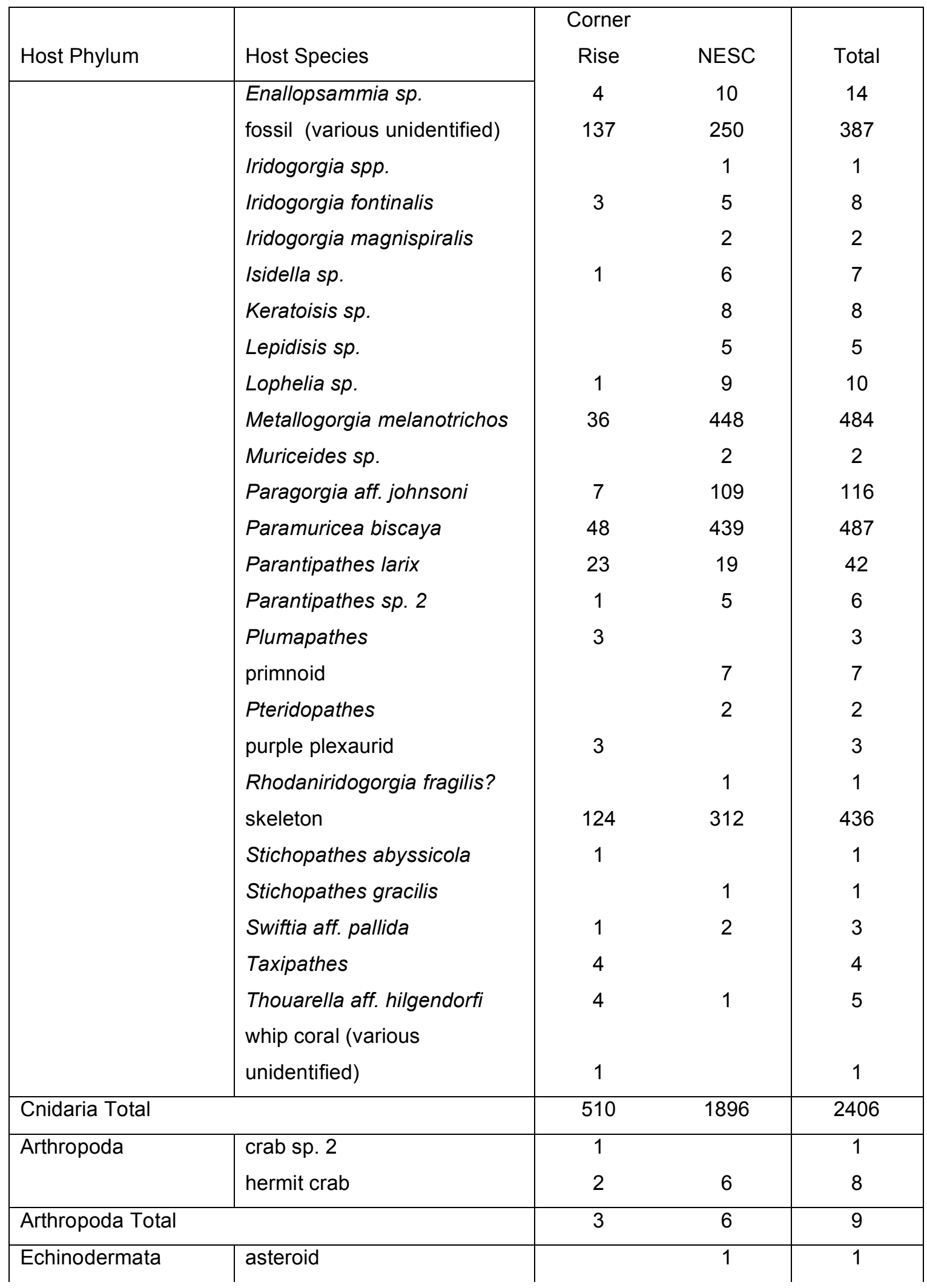


Table 3. cont,

\begin{tabular}{|c|c|c|c|c|}
\hline & & Corner & & \\
\hline Host Phylum & Host Species & Rise & NESC & Total \\
\hline & crinoid sp. 4 & 1 & & 1 \\
\hline & crinoid sp. 5 & 2 & 1 & 3 \\
\hline & crinoid sp. 5b & 1 & & 1 \\
\hline & Hygrosoma aff. petersi & 2 & & 2 \\
\hline & Phormosoma sp. & & 1 & 1 \\
\hline & sea urchin sp. 5 & 3 & & 3 \\
\hline Echinodermata & & 9 & 3 & 12 \\
\hline Chordata & Antimora rostrata & 1 & & 1 \\
\hline & Macourus aff. berglax & 1 & & 1 \\
\hline & tunicate & 2 & & 2 \\
\hline Chordata Total & & 4 & & 4 \\
\hline & & Corner & & \\
\hline Host Type & Host & Rise & NESC & Total \\
\hline Anthropogenic & block & & 13 & 13 \\
\hline & brick? & 1 & & 1 \\
\hline & bucket & 2 & & 2 \\
\hline & rope & & 9 & 9 \\
\hline Anthropogenic 7 & & 3 & 22 & 25 \\
\hline Hard Substrate & Hard Substrate & 5070 & 10324 & 15394 \\
\hline Hard Substrate & & 5070 & 10324 & 15394 \\
\hline Soft Sediment & Soft Sediment & 1628 & 786 & 2414 \\
\hline Soft Sediment T & & 1628 & 786 & 2414 \\
\hline Water Column & Water Column & 936 & 426 & 1362 \\
\hline Water Column 7 & & 936 & 426 & 1362 \\
\hline Total & & 8319 & 13655 & 21974 \\
\hline
\end{tabular}


Table 4. Species occurrence on hosts $(>50 \%,>20$ observations).

\begin{tabular}{|c|c|c|c|c|c|c|c|}
\hline HostlOrg & Hydroid sp. 2 & $\begin{array}{c}\text { Ophioplinthaca } \\
\text { abyssalis }\end{array}$ & $\begin{array}{c}\text { Asteroschema } \\
\text { clavigera }\end{array}$ & $\begin{array}{c}\text { Ophiocreas } \\
\text { oedipus }\end{array}$ & \begin{tabular}{|c|} 
Uroptychus sp. \\
2
\end{tabular} & \begin{tabular}{|c|}
$B$. \\
serratipalma
\end{tabular} & $\begin{array}{l}\text { Octopus } \\
\text { egg case }\end{array}$ \\
\hline Hard Substrate & $10.2 \%$ & $1.2 \%$ & 0 & 0 & 0 & 0 & 0 \\
\hline Acanella sp. & 0 & 0 & 0 & 0 & 0 & 0 & $16 \%$ \\
\hline Acanthogorgia sp. & 0 & $0.1 \%$ & 0 & 0 & 0 & 0 & 0 \\
\hline Candidella sp. & 0 & $91.9 \%$ & 0 & 0 & 0 & 0 & 0 \\
\hline Chrysogorgia sp. & $0.1 \%$ & 0 & 0 & 0 & 0 & $75 \%$ & $60 \%$ \\
\hline Chrysogorgia sp. 1 & 0 & 0 & 0 & 0 & 0 & $15 \%$ & $8 \%$ \\
\hline \begin{tabular}{|c|} 
Chrysogorgia sp. 2 \\
\end{tabular} & 0 & 0 & 0 & 0 & 0 & $10 \%$ & $4 \%$ \\
\hline Corallium sp. & 0 & $5.3 \%$ & 0 & 0 & 0 & 0 & 0 \\
\hline Enallopsammia sp. & $15.8 \%$ & 0 & 0 & 0 & 0 & 0 & 0 \\
\hline Fossil coral & $0.6 \%$ & 0 & 0 & 0 & 0 & 0 & 0 \\
\hline Metallogorgia sp. & 0 & 0 & 0 & $100 \%$ & 0 & 0 & $8 \%$ \\
\hline Paramuricea sp. & 0 & 0 & $66.3 \%$ & 0 & 0 & 0 & 0 \\
\hline Paragorgia sp. & $3.5 \%$ & 0 & $33.7 \%$ & 0 & 0 & 0 & 0 \\
\hline Parantipathes sp. 1 & 0 & 0 & 0 & 0 & $84.2 \%$ & 0 & 0 \\
\hline Parantipathes sp. 2 & 0 & $0.2 \%$ & 0 & 0 & $15.8 \%$ & 0 & 0 \\
\hline Coral Skeleton & $69.5 \%$ & $1.3 \%$ & 0 & 0 & 0 & 0 & $4 \%$ \\
\hline Sponge sp. & $0.1 \%$ & 0 & 0 & 0 & 0 & 0 & 0 \\
\hline Sponge sp. 11 & $0.1 \%$ & 0 & 0 & 0 & 0 & 0 & 0 \\
\hline Echinoid sp. 5 & $0.1 \%$ & 0 & 0 & 0 & 0 & 0 & 0 \\
\hline Taxipathes sp. & 0 & 0 & 0 & 0 & 0 & 0 & 0 \\
\hline $\begin{array}{l}\text { Total } \\
\text { Observations }\end{array}$ & 1263 & 1285 & 726 & 480 & 74 & 20 & 25 \\
\hline
\end{tabular}




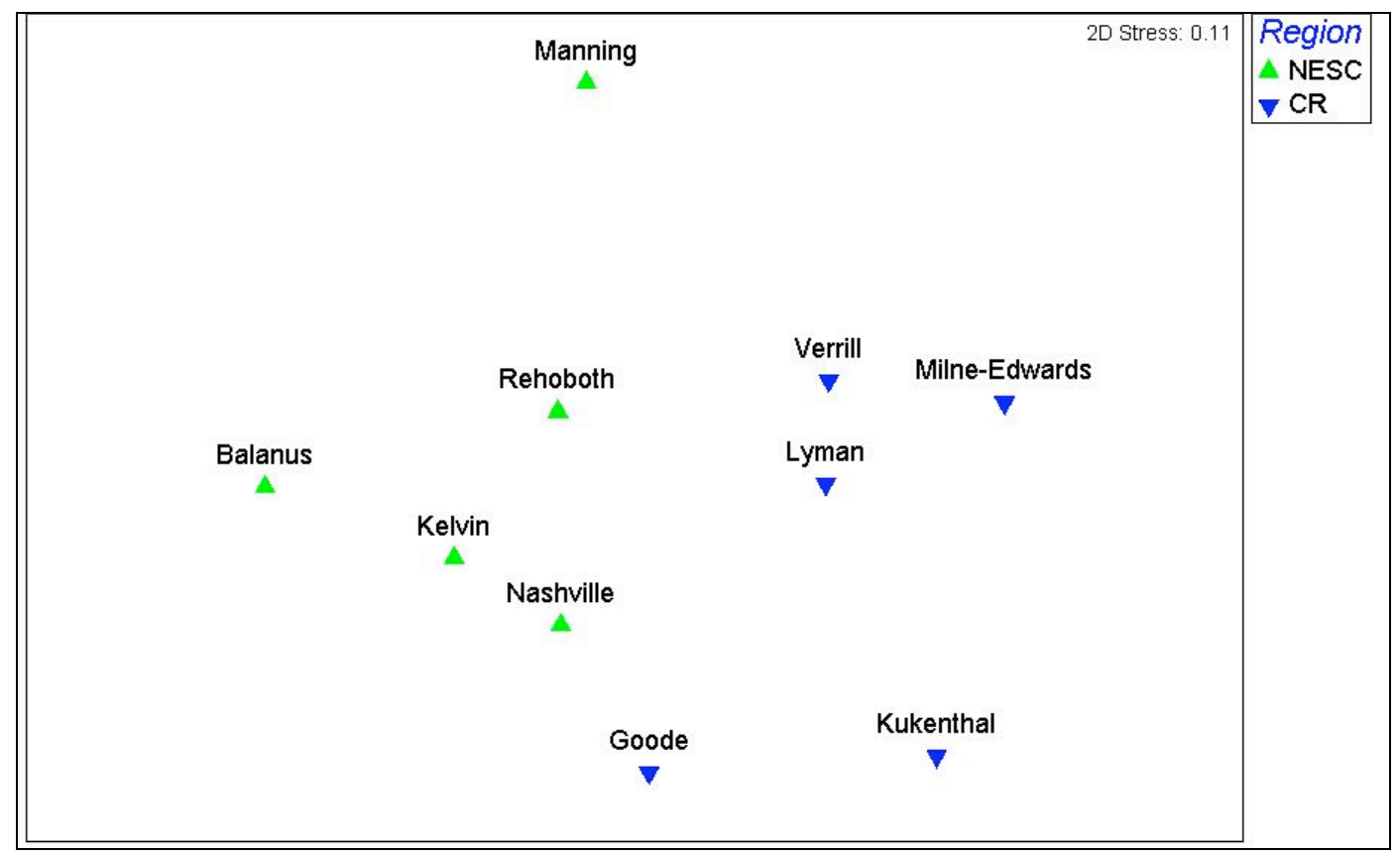

Figure 14. Multidimensional scaling of faunal assemblages for dives using the Bray-Curtis similarity index. Ordination points superimposed by geographic region. $\mathrm{CR}=$ Corner $\mathrm{Rise}, \mathrm{NESC}=\mathrm{New}$ England seamount chain.

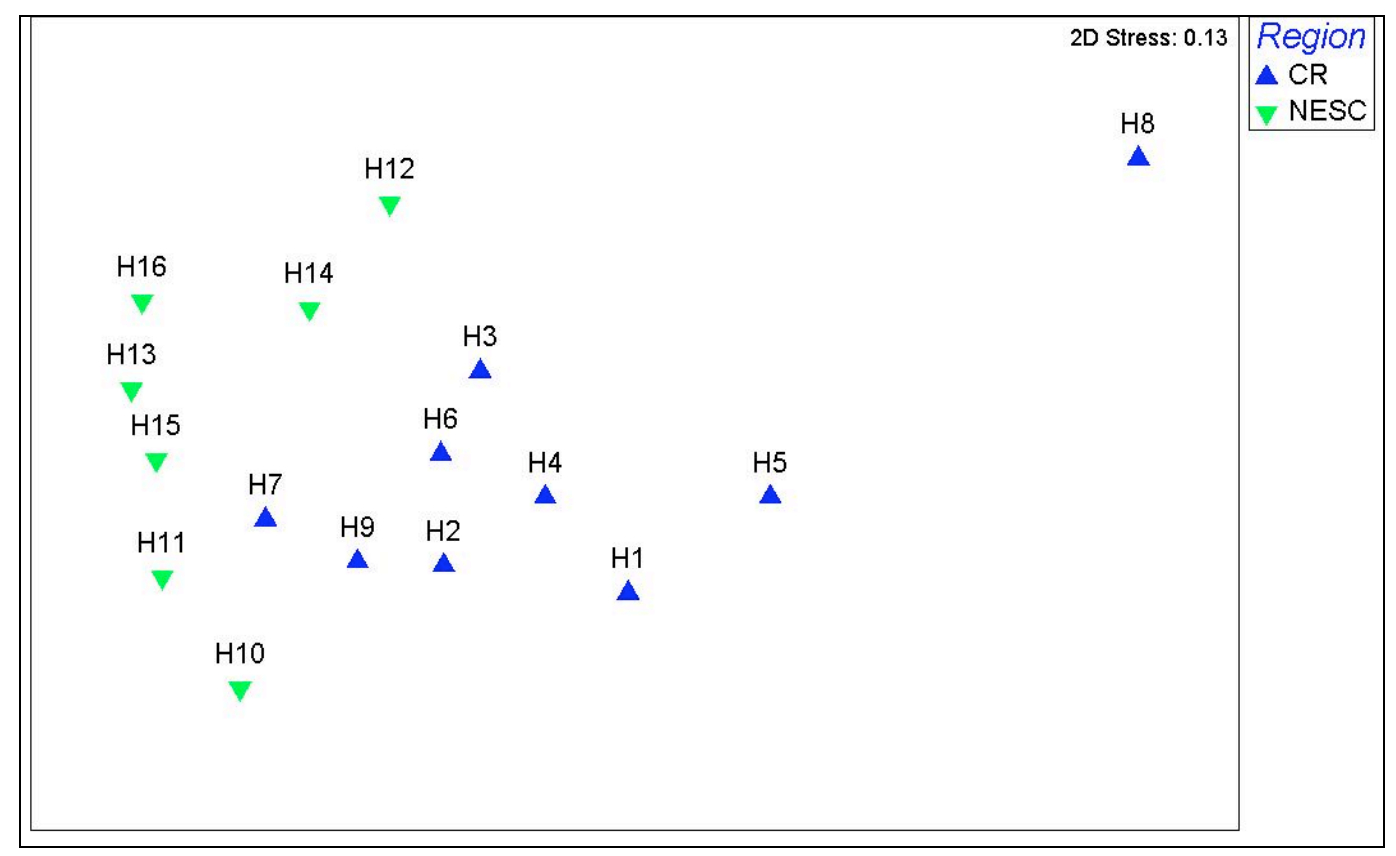

Figure 15. Multidimensional scaling of faunal assemblages for dives using the Bray-Curtis similarity index. Ordination points superimposed by geographic region. $C R=$ Corner Rise, $N E S C=$ New England seamount chain. 


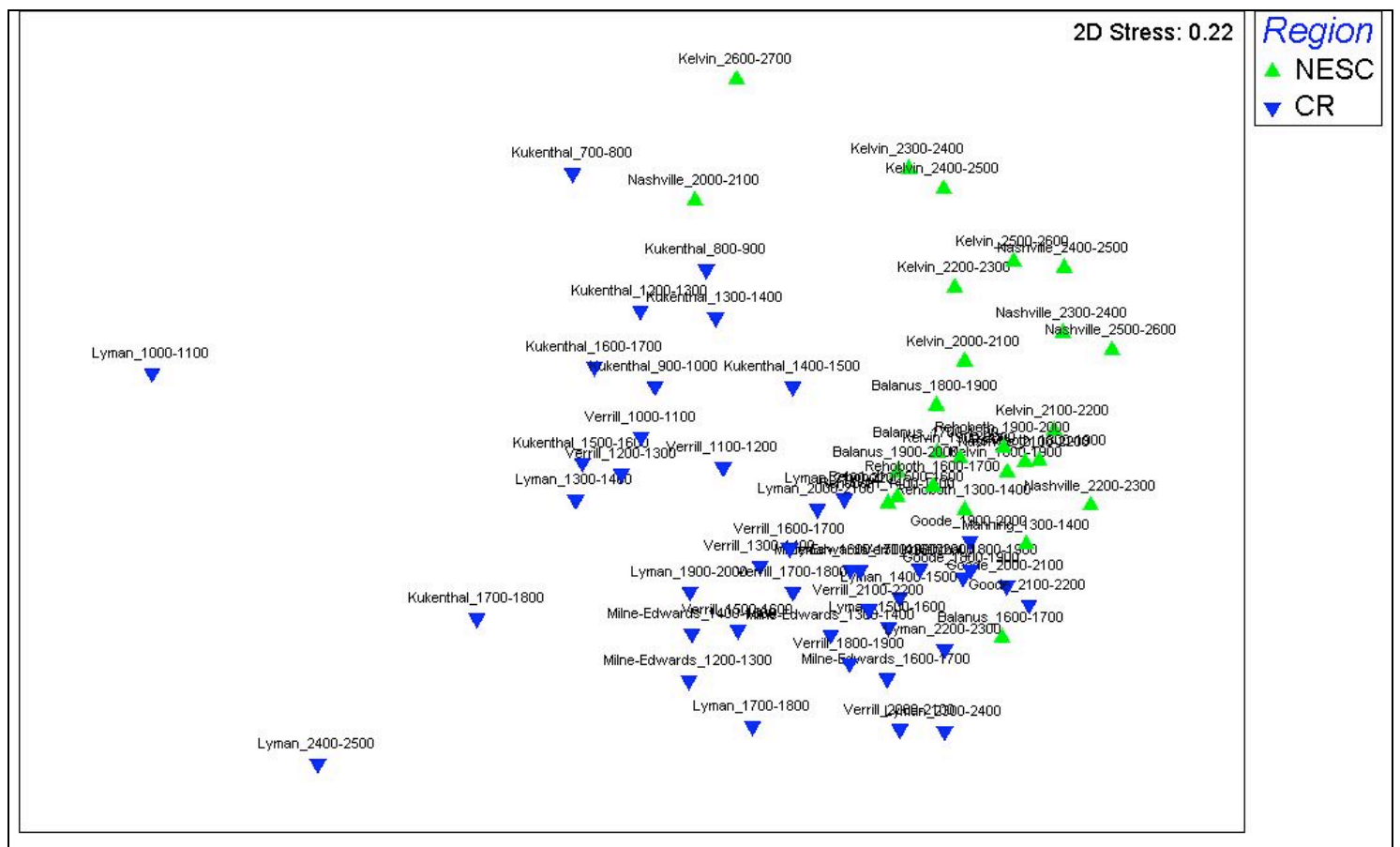

Figure 16. Multidimensional scaling of faunal assemblages for $100 \mathrm{~m}$ depth bins within a seamount using the Bray-Curtis similarity index. Ordination points superimposed by geographic region. $\mathbf{C R}=$ Corner Rise, NESC = New England seamount chain . 


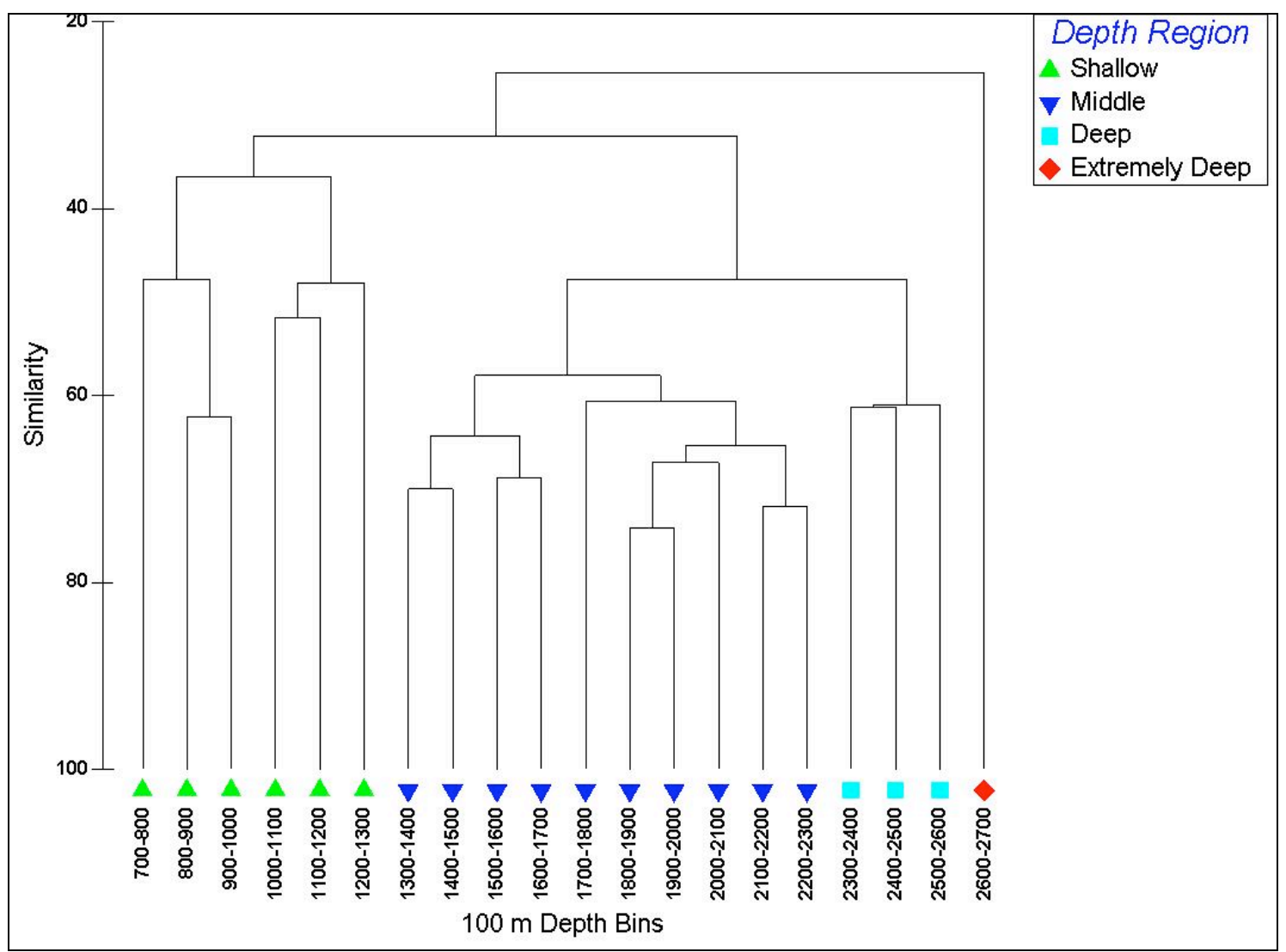

Figure 17. Dendrogram of similarity among $100 \mathrm{~m}$ depth bins in faunal assemblages from the New England and Corner Rise seamounts, produced with cluster analysis using the Bray-Curtis similarity index. 


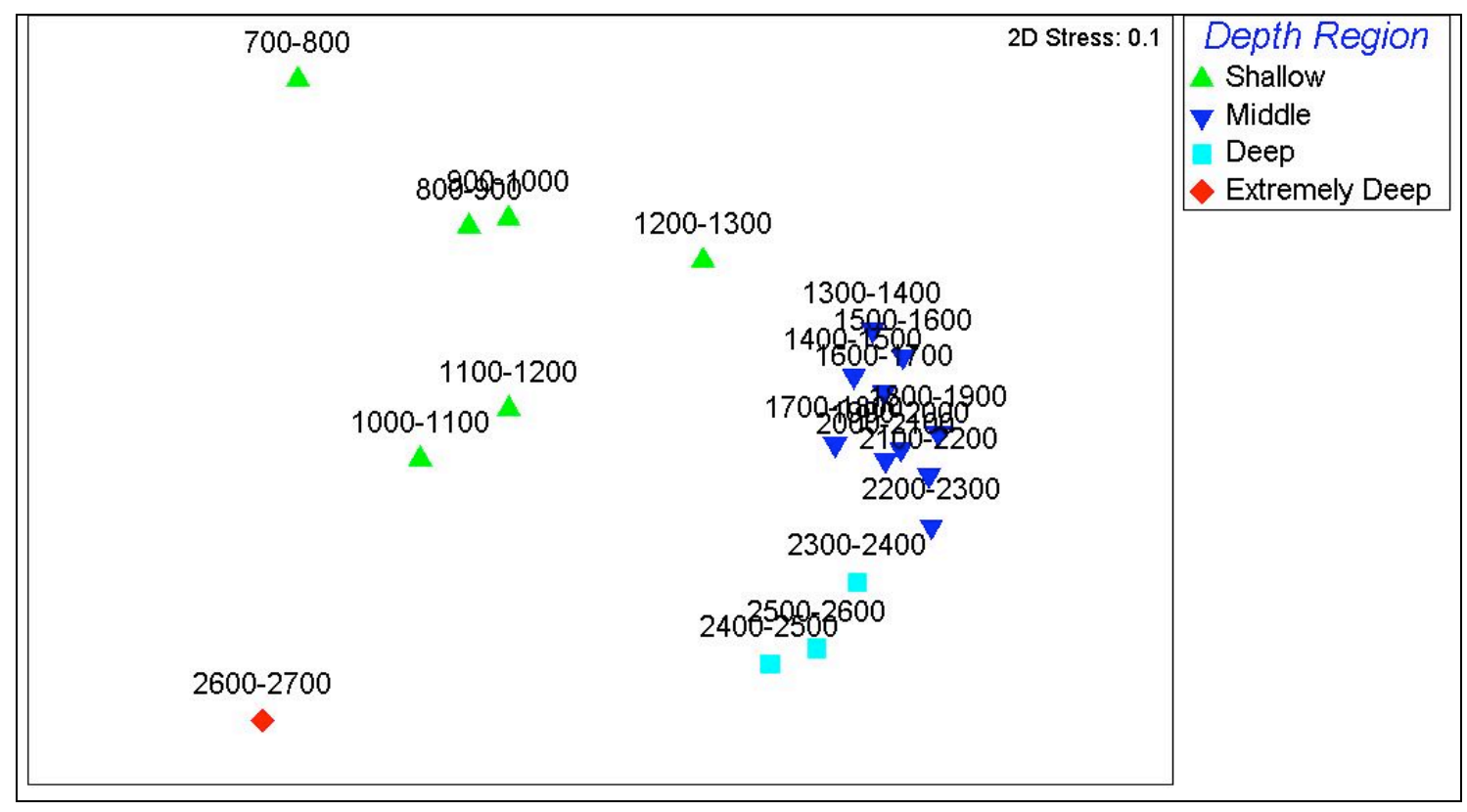

Figure 18. Multidimensional scaling of faunal assemblages for $100 \mathrm{~m}$ depth bins using the BrayCurtis similarity index. Ordination points superimposed by depth region: 1) Shallow $<1300 \mathrm{~m}, 2$ ) 1400 m < Middle < 2300 m, 3) 2300 m < Deep < 2600 m, 4) Extremely Deep > 2600m.

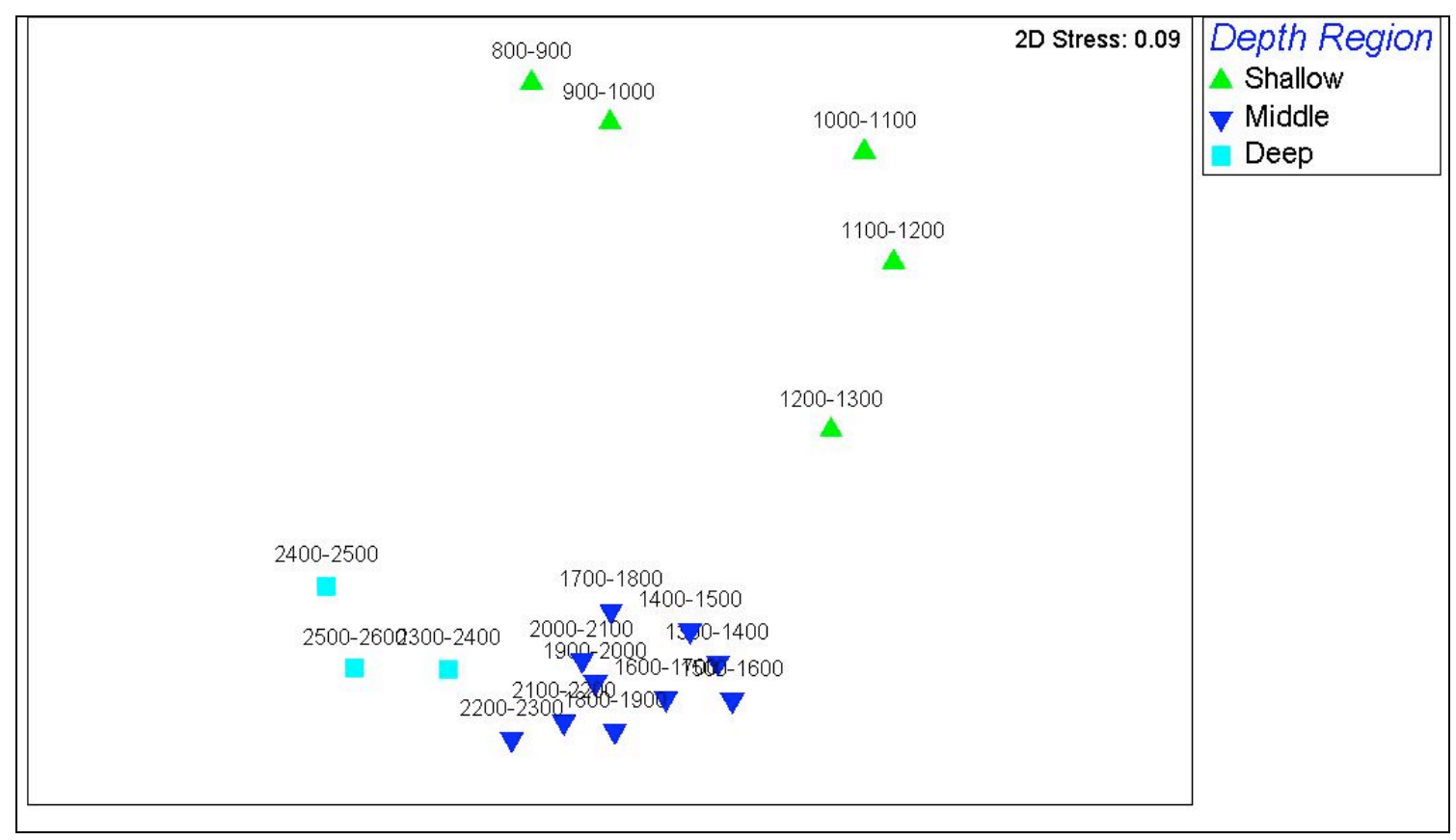

Figure 19. Multidimensional scaling of faunal assemblages for $100 \mathrm{~m}$ depth bins using the BrayCurtis similarity index. Ordination points superimposed by depth region: 1) Shallow $<1300 \mathrm{~m}, 2$ ) $1400 \mathrm{~m}<$ Middle $>2400 \mathrm{~m}$; 3) Deep $>2300 \mathrm{~m}$. 
Table 5. Results of SIMPER analysis of Middle and Deep depth regions showing fauna that account for top $20 \%$ of differences between the two regions.

\begin{tabular}{lllll} 
& \multicolumn{2}{l}{ Middle Depth Region } & Deep Depth Region & \\
\cline { 2 - 3 } Species & Avg. Abundance & Avg. Abundance & Contribution \% & Cumulative \% \\
\hline O. chelys & 1.11 & 0.00 & 2.28 & 2.28 \\
Farrea sp. & 1.29 & 0.34 & 1.98 & 4.26 \\
A. clavigera & 0.94 & 0.00 & 1.94 & 6.19 \\
M. & 0.93 & 0.00 & 1.91 & 8.12 \\
melanotrichos & & & & \\
O. oedipus & 0.92 & 0.00 & 1.91 & 10.03 \\
Crinoid & 1.52 & 0.64 & 1.85 & 11.88 \\
Acanella sp. & 0.87 & 0.00 & 1.79 & 13.67 \\
Sea urchin & 0.67 & 1.50 & 1.71 & 15.38 \\
Paramuricea sp. & 1.01 & 0.24 & 1.57 & 16.95 \\
Geodia sp. & 0.91 & 1.64 & 1.53 & 18.47 \\
C. imbricata & 0.73 & 0.00 & 1.50 & 19.97 \\
O. abyssalis & 0.71 & 0.00 & 1.46 & 21.43 \\
\hline
\end{tabular}




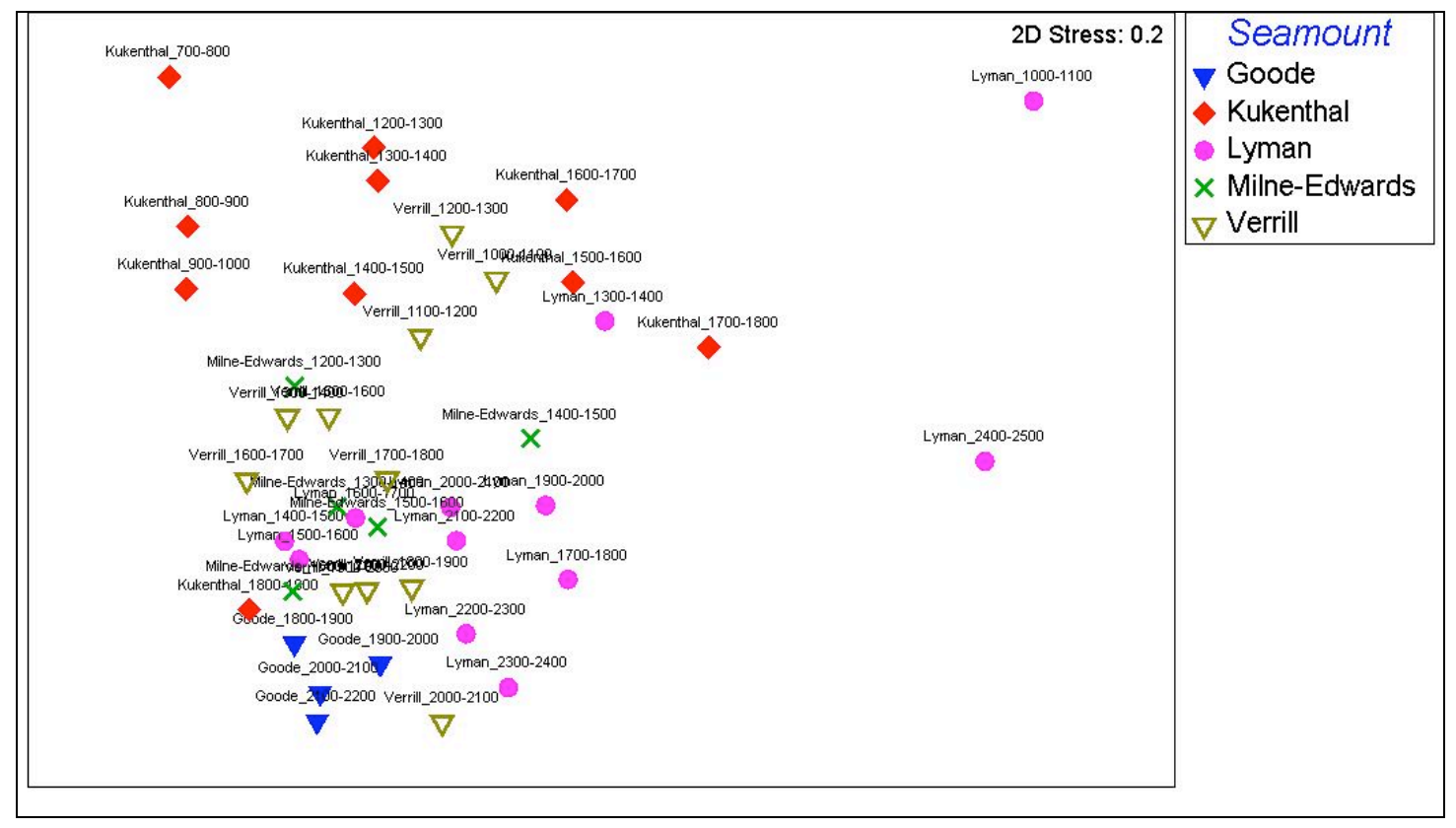

Figure 20. Multidimensional scaling of faunal assemblages for $100 \mathrm{~m}$ depth bins within seamounts in the Corner Rise using the Bray-Curtis similarity index. Ordination points superimposed by seamount.

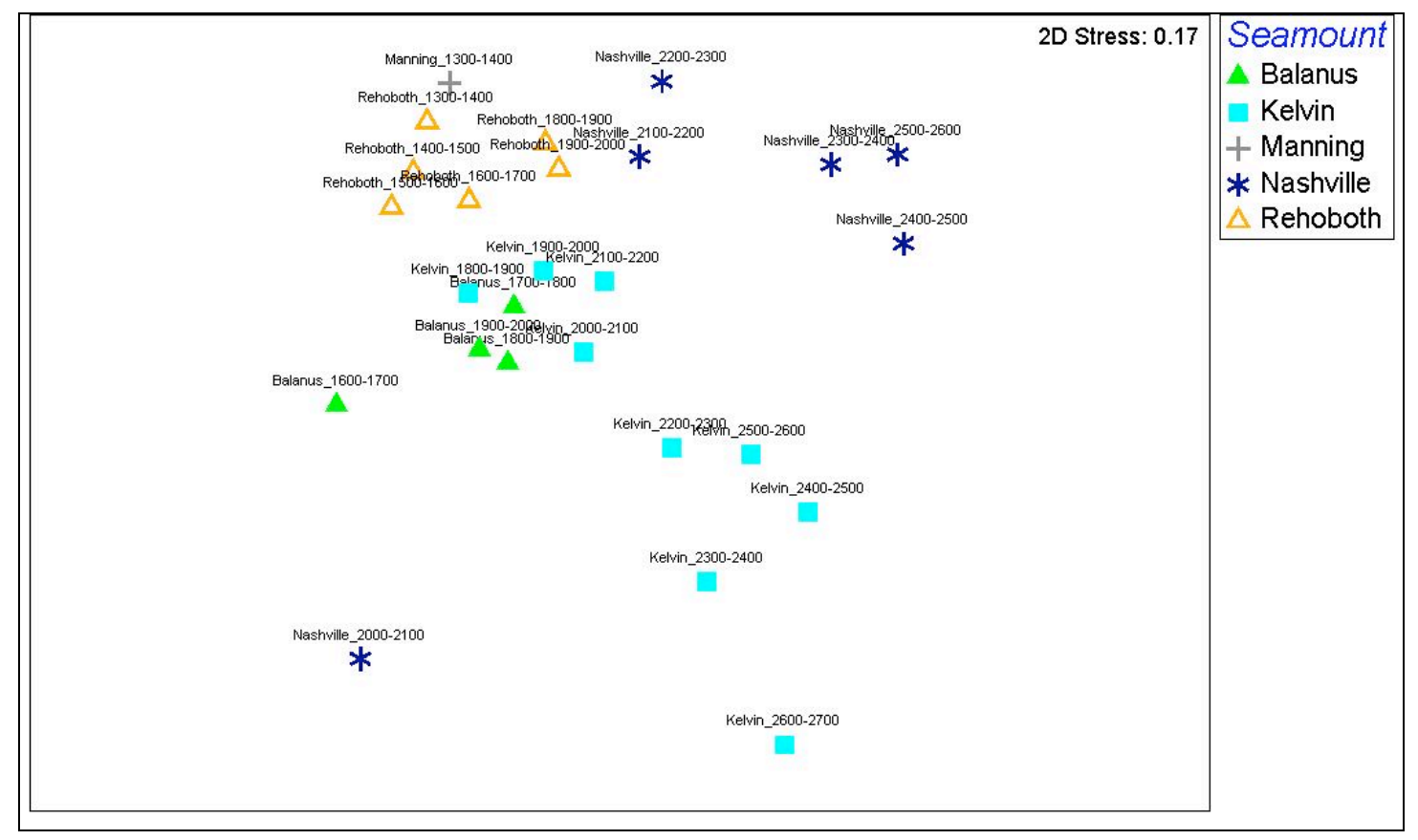

Figure 21. Multidimensional scaling of faunal assemblages for $100 \mathrm{~m}$ depth bins within seamounts in the New England seamount chain using the Bray-Curtis similarity index. Ordination points superimposed by seamount. 


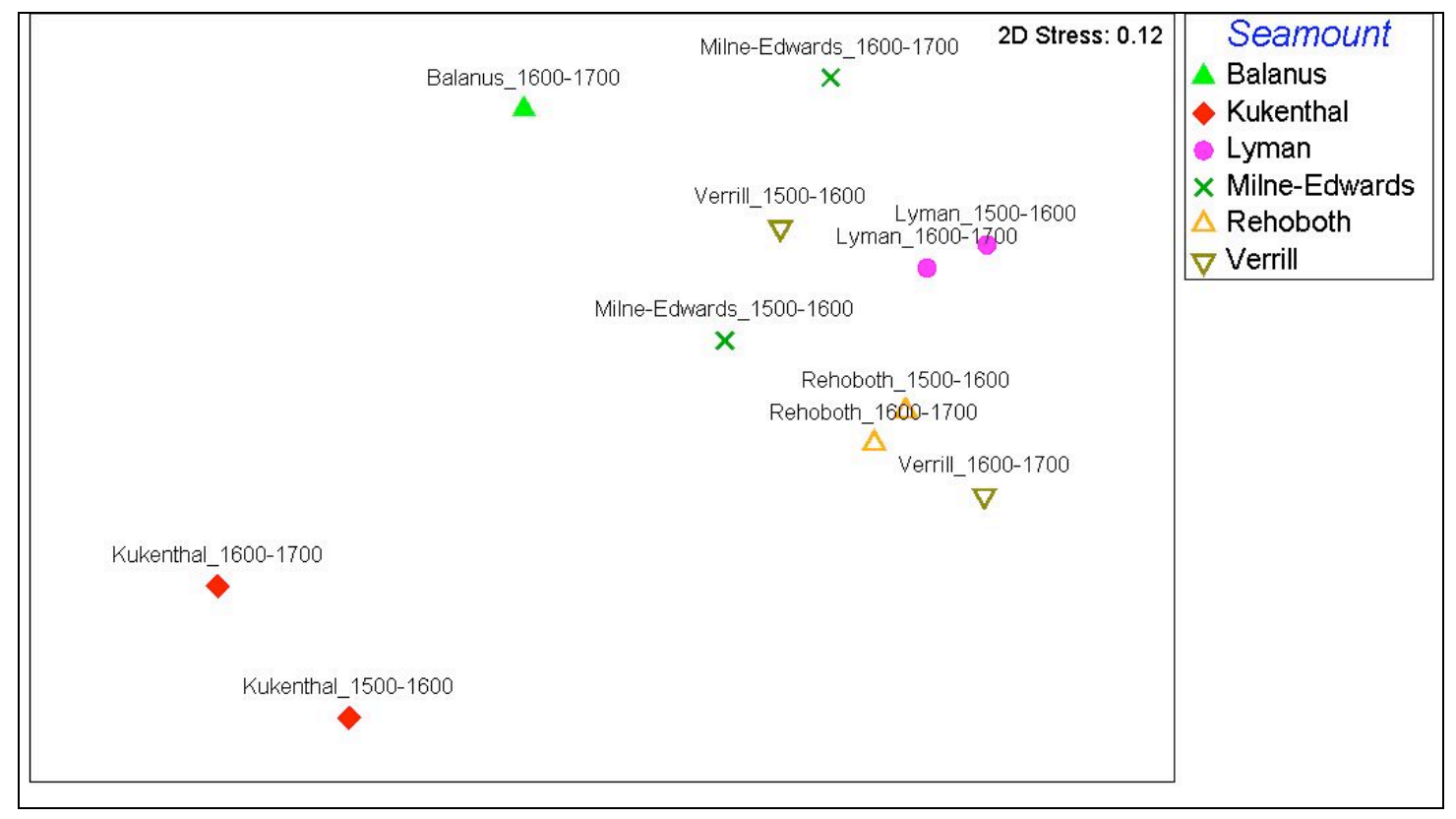

Figure 22. Multidimensional scaling of faunal assemblages for $100 \mathrm{~m}$ depth bins within seamounts within 1500-1700 $\mathrm{m}$ using the Bray-Curtis similarity index. Ordination points superimposed by seamount.

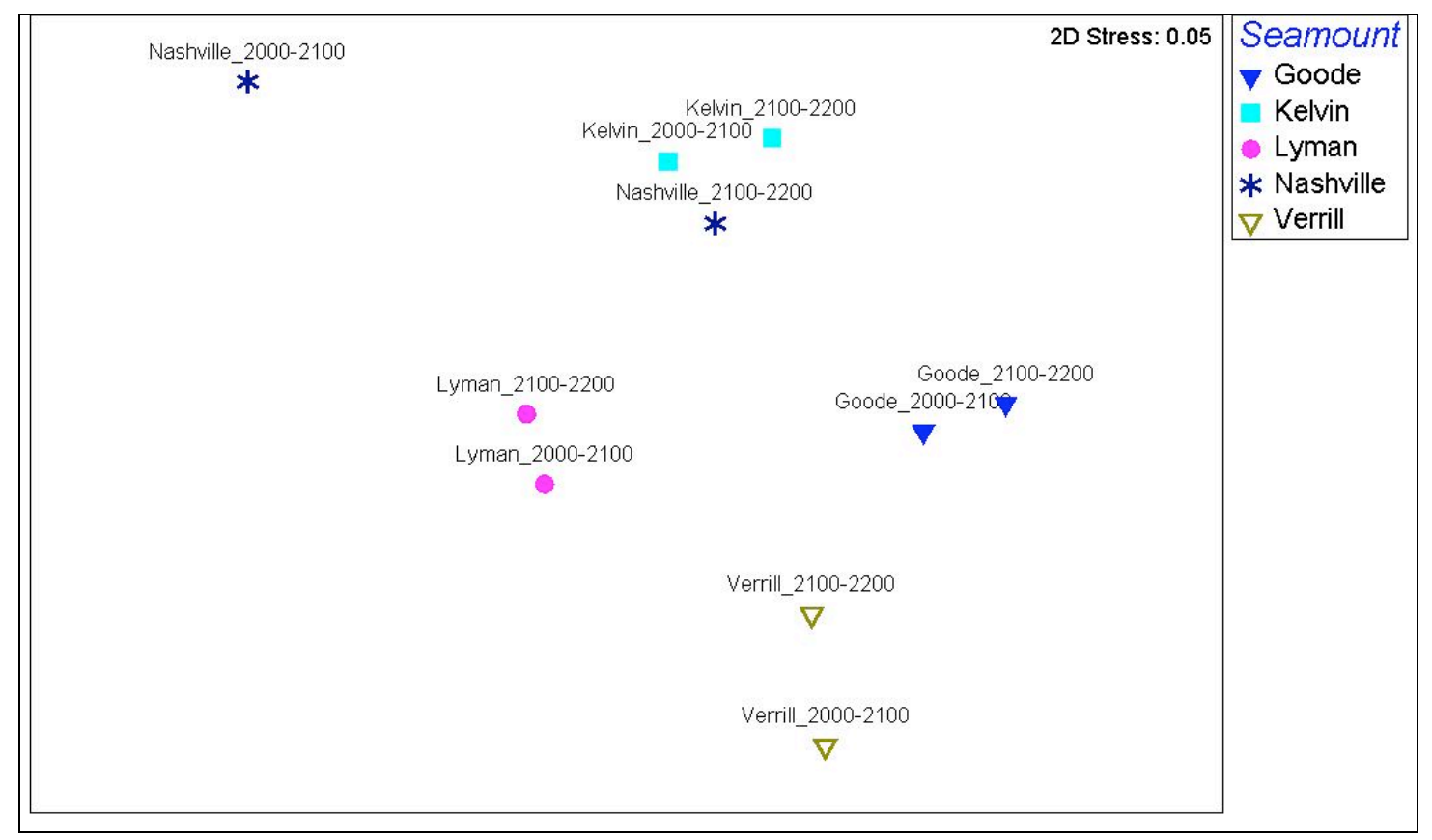

Figure 23. Multidimensional scaling of faunal assemblages for $100 \mathrm{~m}$ depth bins within seamounts within 2000-2200 $\mathrm{m}$ using the Bray-Curtis similarity index. Ordination points superimposed by seamount. 


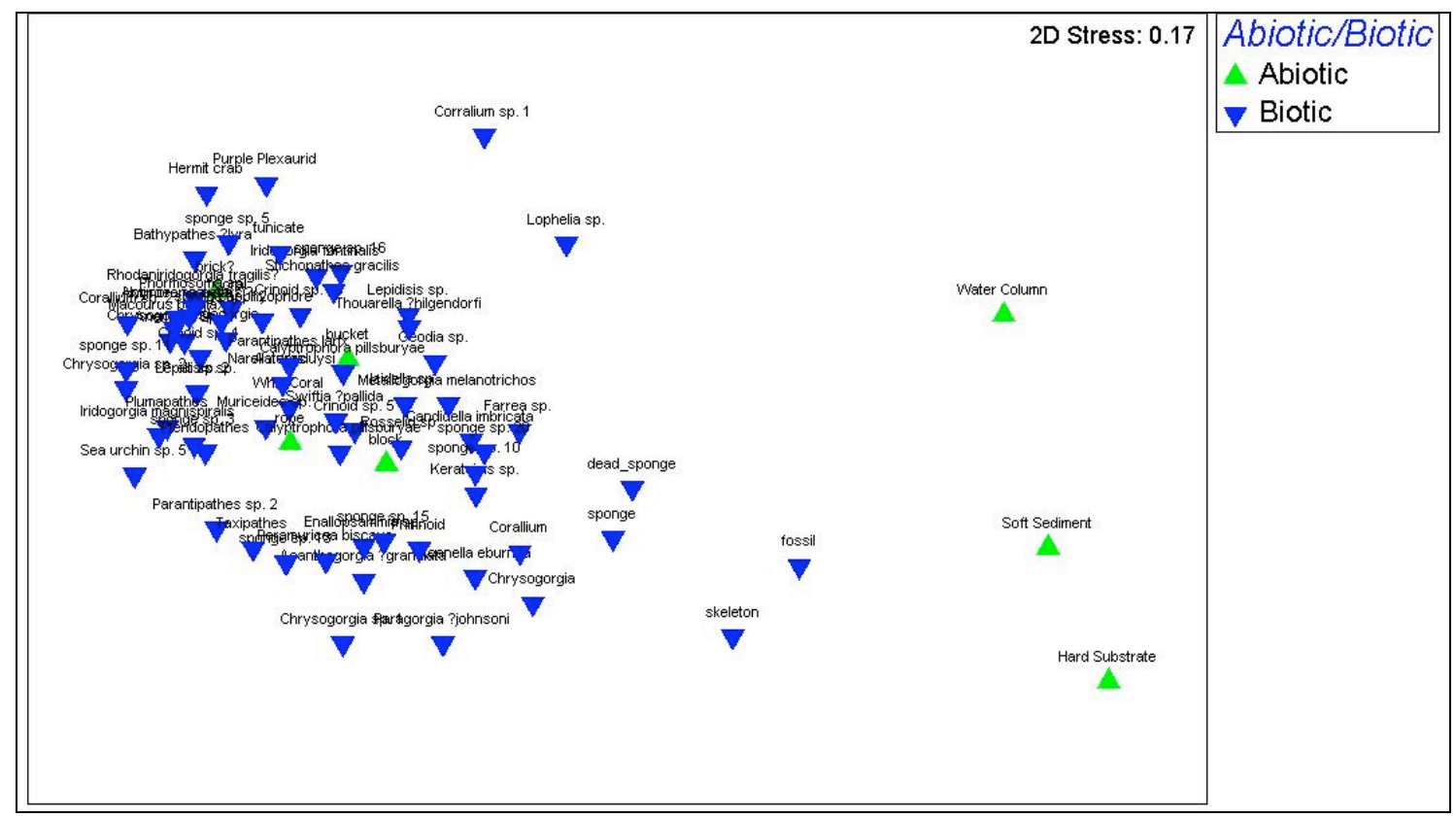

Figure 24. Multidimensional scaling of faunal assemblages for substrates using the Bray-Curtis similarity index. Ordination points superimposed by abiotic or biotic substrate type.

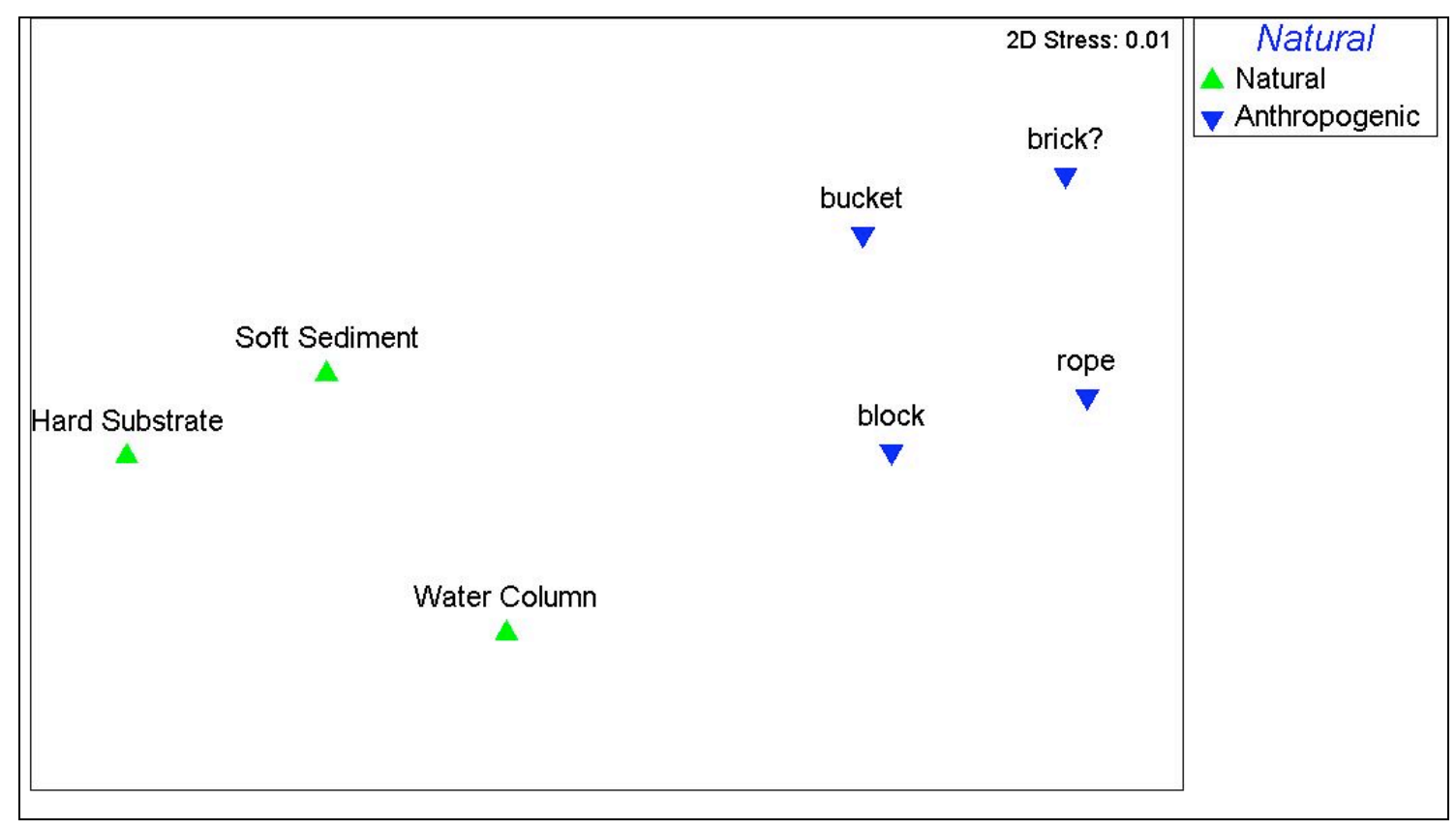

Figure 25. Multidimensional scaling of faunal assemblages for abiotic substrates using the BrayCurtis similarity index. Ordination points superimposed by natural or anthropogenic substrate type. 


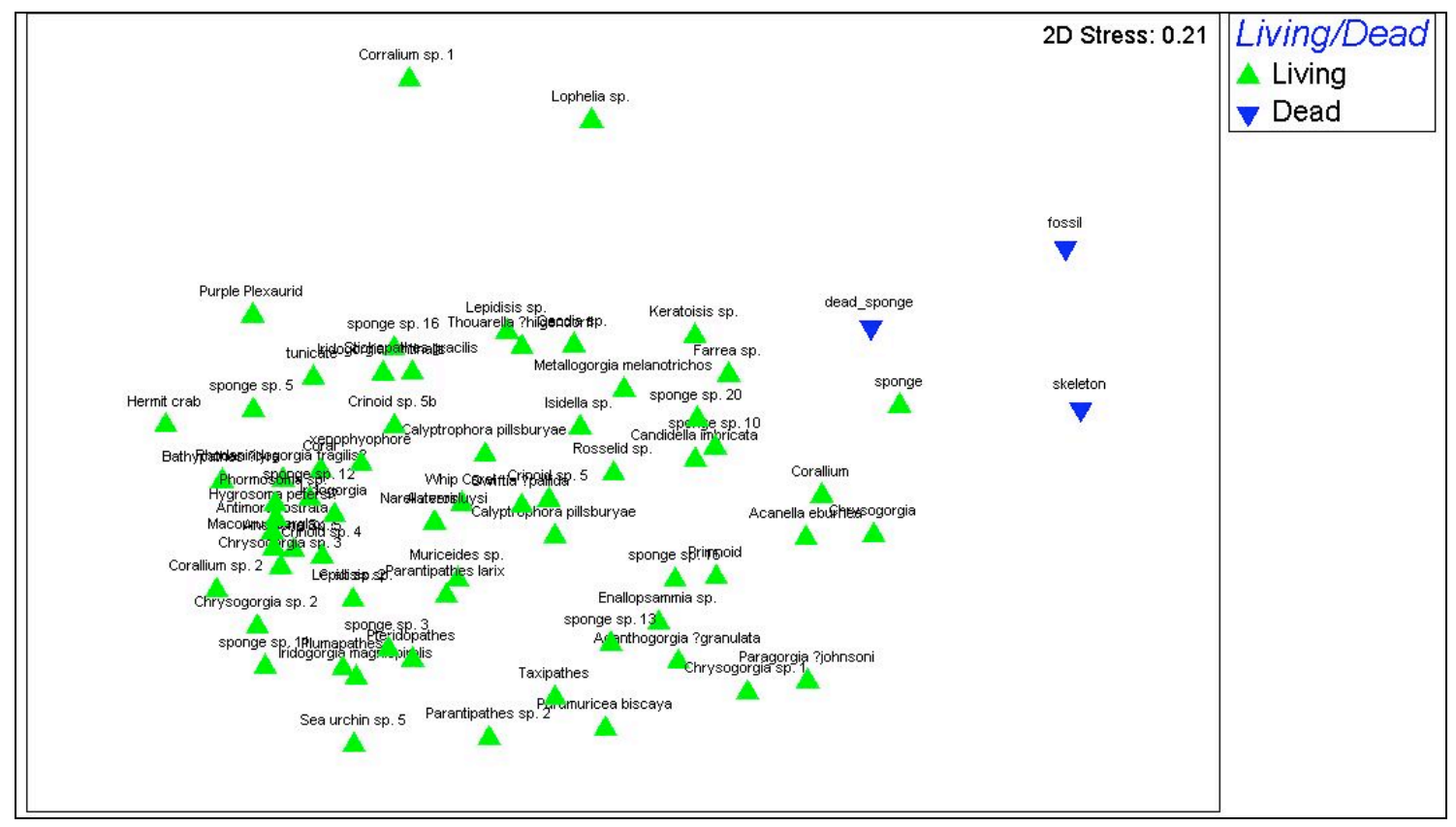

Figure 26. Multidimensional scaling of faunal assemblages for biotic substrates using the Bray-Curtis similarity index. Ordination points superimposed by living or dead substrates.

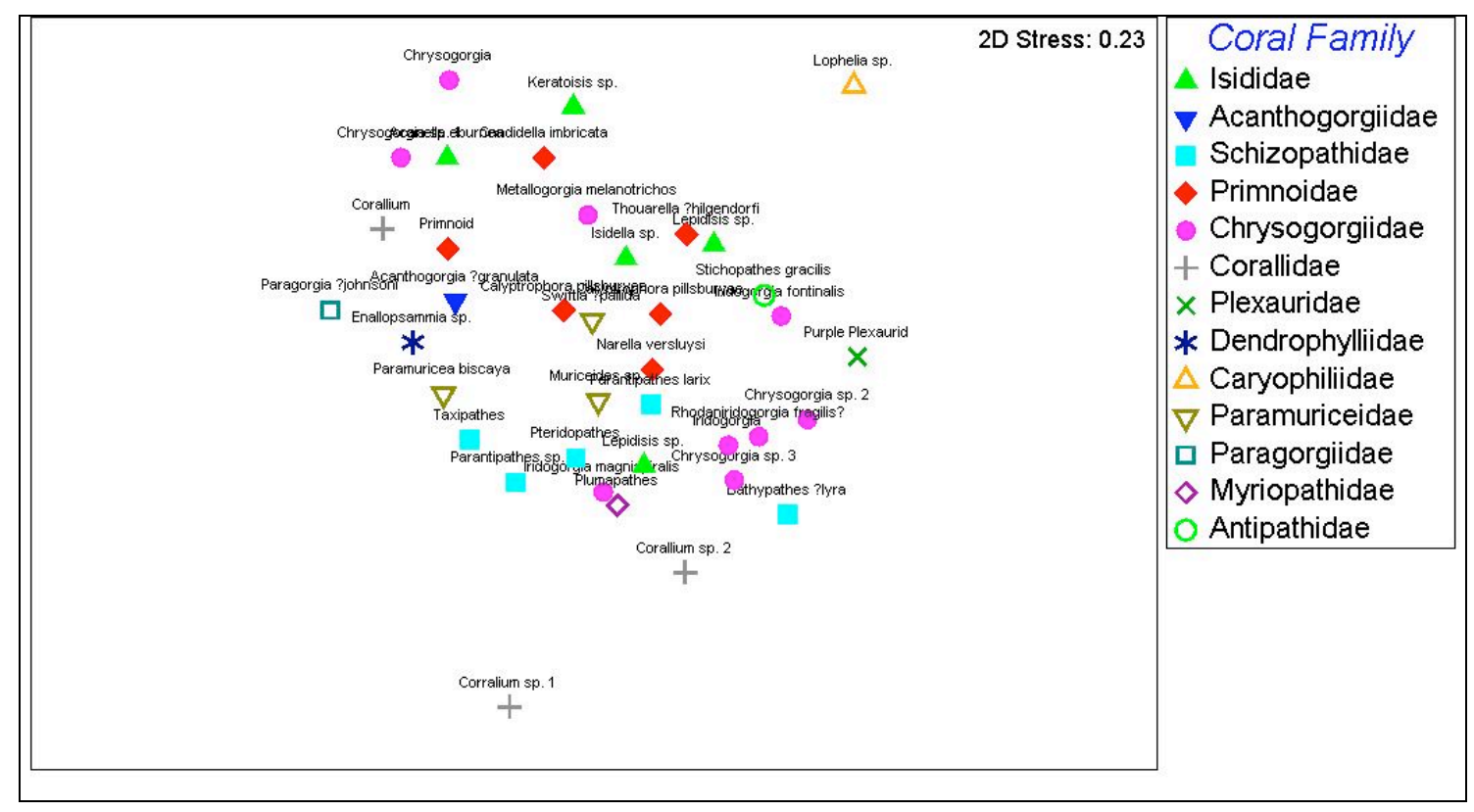

Figure 27. Multidimensional scaling of faunal assemblages for coral hosts using the Bray-Curtis similarity index. Ordination points superimposed by coral family. 


\section{DISCUSSION}

\section{Taxonomic composition}

The most abundant phyla observed in this study were the Porifera (sponges), Cnidaria (mainly corals), and the Echinodermata (ophiuroids, sea urchin, and holothurians). This result differs from previous studies where the most reported group among seamounts is the Crustacea according to SeamountsOnline, an online database of seamounts information (Stocks 2004). This difference may be due to the different methods of collection used to create the SeamountsOnline database, and also a sampling bias due to the commercial value of crab and shrimp, which may have been of particular interest in previous seamount surveys (Stocks 2004). Also, a study of epibenthic megafauna on Horizon Guyot and Magellan Rise found that xenophyophores dominated the fauna (Kaufmann et al. 1989). This study, however, focused on the summit cap and cap perimeter of the seamounts, whereas our study included dives from many different parts of a seamount, including the summit and the sides.

Despite these differences, the fauna observed on the seamounts were dominated by suspension feeders, which is consistent with previous studies of seamount fauna (Metaxas and Davis 2005; Rogers 1994; Stocks 2004). The benthic biomass is often dominated by large suspension feeders that provide important habitat for smaller, mobile invertebrates (Samadi et al. 2007). Suspension feeders accounted for $52 \%$ of a global compilation of benthic seamount species (Wilson and Kaufmann 1987). When broken down by region, these values fall with suspension feeders accounting for $15 \%$ in the Eastern Pacific, $27 \%$ on the Tasmanian seamounts, and $22 \%$ on the Howe and Norfolk Ridge in the southwest Pacific (Parin et al. 1997; Samadi et al. 2007).

The dominance of filter feeders is thought to be related with the interaction of seamounts with the surrounding currents. Currents can help expose hard substrate to provide habitat for sessile filter feeders such as corals and sponges by removing 
sediment, which otherwise dominates the deep sea benthic habitat (Gage and Tyler 1991; Stocks 2004). Also, current velocity increases around edges or on topographic highs, thereby increasing the encounter rate of nutrients for filter feeders and nutrient availability (Genin et al. 1986; Metaxas and Davis 2005; Mortensen and Buhl-Mortensen 2004). The interaction between seamounts and currents also form Taylor columns, regions with closed streamlines that form as ocean currents pass and interact with seamounts, which result in closed eddies and localized upwelling (Mullineaux and Mills 1997; Rogers 1994; Taylor 1917). These closed recirculation cells have been suggested as a mechanism for increased productivity and isolation.

\section{Regional Patterns}

In the comparison of community composition within the study area, the communities of the Corner Rise and New England seamount chain were found to be significantly different. This regional difference was seen in the significant numbers of unique taxa observed in the two regions ( 75 morphospecies unique to the Corner Rise seamounts and 60 morphospecies unique to the New England seamounts), as well as in the multivariate analyses of community composition performed on the regional scale. Also, three phyla were observed only on the Corner Rise seamounts: the Hemichordata, the Nematoda, and the Sipuncula; however, these were merely single observations. These are very small taxa that would not be expected to have been observed in this kind of study.

This pattern of regional differences was also observed between two ridge systems in southwest Pacific seamounts, and also in regional differences found in megafauna assemblages associated with deep-water gorgonian corals in different sites in the Northeast Channel in Canada (Metaxas and Davis 2005; Richer De Forges et al. 2000).

The observation of unique taxa from the different seamount regions in the North Atlantic may be an indication of endemism, which is consistent with previous studies showing seamounts to be areas with a high degree of endemism (Rogers 1994). Wilson 
and Kaufman (1987) estimated that 12\% of all fish found on seamounts were endemic, while the invertebrate fauna was estimated to have a $15 \%$ level of endemism. Richer de Forges et al. (2000) found that 29-34\% of the macro- and megafauna of seamounts found in the Tasman Sea and southeast Coral Sea were potential seamount endemics. Even higher rates were found on the Nasca and Sala-y-Gomez seamount chains off the coast of Chile, which had endemism levels for fish of $44 \%$ and $52 \%$ for invertebrates (Parin et al. 1997).

These regional differences may be caused by a variety of factors. There are several characteristics of seamounts that may prevent dispersal of seamount fauna. They are unique hard substrate habitats due to their formation from volcanic processes, making them isolated islands within a deep-sea sometimes dominated by soft sediment habitat. Fauna dependent upon hard substrate would be required to travel across this distance to find other suitable habitat to disperse to and settle on, making distance a factor to consider. This would also be dependent upon the geologic history of the seamount, whether they were formed as a linear chain, in clusters, or even as solitary seamounts.

The North Atlantic seamounts are thought to be a result of the movement of the North Atlantic plate over the Great Meteor mantle plume, forming both the New England seamount chain and the Corner Rise seamounts (Zheng and Arkani-Hamed 2002). However, there are distinct age differences between the two seamount groups. ${ }^{40} \mathrm{Ar} /{ }^{39} \mathrm{Ar}$ isotope dating has found that the oldest New England seamount, Bear seamount, was formed about 103 mya and the youngest New England seamount, Nashville seamount, was formed approximately 82 mya. The New England seamounts form a roughly linear chain stretching southeast from Bear seamount to Nashville seamount. The Corner Rise seamounts are estimated to have been formed approximately 75 mya and are distinct from the New England seamounts (Duncan 1984; Zheng and Arkani-Hamed 2002). This distinct geological history between the two seamount groups may account for the distinct faunal assemblages observed in this study.

The hydrographic regime of the region may also be important as seamounts have been shown to influence the currents around them in ways that could cause isolation, 
such as through the formation of Taylor columns and isolating eddies (Ezer 1994; Mullineaux and Mills 1997; Qiu 1994; Rogers 1994; Taylor 1917). Modeling studies of current flow around the North Atlantic seamounts show the formation of such eddies that could isolate the two seamount groups (Qiu 1994).

\section{Depth}

Cluster analysis and multivariate analyses of community composition suggest four faunal assemblages based on depth with breaks at $1300 \mathrm{~m}, 2300 \mathrm{~m}$, and $2600 \mathrm{~m}$. Similar distinct faunal assemblages based on depth have also been observed on Patton seamount in the Gulf of Alaska (Hoff and Stevens 2005). Depth has also been found to be an important factor in structuring seamount communities (Samadi et al. 2007). Many factors co-vary with depth, which may determine the vertical distribution of fauna. In the Northeast Atlantic, maximum depths of deep-water corals often reflect changes in the depths of water masses that have temperatures suitable for the corals. This was seen for deep-water gorgonian corals in the Northeast Channel of Canada (Mortensen and BuhlMortensen 2004).

Similar faunal zonation by depth has been seen in other studies. Important faunal boundaries are thought to occur globally around 1000-1400 m depth (Howell et al. 2002), coinciding with the $1300 \mathrm{~m}$ observed in this study. Similar faunal boundaries have been seen in asteroids in the North Atlantic at $1100 \mathrm{~m}$ (Howell et al. 2002), 1200-1300 m for decapod crustaceans (Cartes and Sarda 1993), $1000 \mathrm{~m}$ for cerianthid anemones (Shepard et al. 1986). This boundary has been attributed to slope gradient, which is related to the formation of enhanced bottom currents through internal tides and can effect sediment transport and substratum type (Howell et al. 2002). A study of the deep-sea megabenthic fauna south of New England also showed a similar break separating two distinct faunal assemblages between $1290 \mathrm{~m}$ and $1380 \mathrm{~m}$ (Haedrich et al. 1980). This same study showed a similar break between $1947 \mathrm{~m}-2116 \mathrm{~m}$ which may be similar to the $2300 \mathrm{~m}$ break observed in this study, however there were only two dives in the $2300-2600 \mathrm{~m}$ 
range and this result may be a sampling artifact. The $2300 \mathrm{~m}$ break also coincides with a peak in diversity for soft-bottom invertebrate megafauna in the northwest Atlantic between 1900 - $2300 \mathrm{~m}$ (Howell et al. 2002; Rex 1981). The $2600 \mathrm{~m}$ break observed in this study is similar to a region of pronounced changes in fauna seen in other studies as well. This break coincides with a boundary of a well-defined intermediate region of 2500-3500 m where bathyal fauna meet, and overlap with, abyssal fauna (Haedrich et al. 1980; Howell et al. 2002). The result of this depth zonation pattern is probably the result of a complex combination of physical factors and biological interactions (Gage and Tyler 1991).

The results of the SIMPER analysis revealed that the difference between the middle and deep depth regions was accounted for by the disappearance of seven of the twelve taxa that were responsible for $20 \%$ of the difference between the faunal assemblages in these two depth regions. The four ophiuroids $O$. chelys, A. clavigera, $O$. oedipus, and O. abyssalis and the three corals M. melanotrichos, Acanella sp., and $C$. imbricata were present in the middle depth region but absent from the deep depth region. This is an indication of the importance of substrate or host availability is important for structuring distinct faunal assemblages as the ophiuroids $O$. oedipus and O. abyssalis were absent as were both of their host corals, $M$. melanotrichos and $C$. imbricat. In addition, Paramuricea sp., one of the hosts for the ophiuroid A. clavigera was less abundant in the deep depth region as well and may explain the absence of this ophiuroid from the deep depth region.

As both geography and depth both seem to play an important role in structuring seamount communities, one concern is the confounding effect one factor may have on the other. Multivariate analyses comparing only those samples from seamounts that share a common depth range showed samples more closely related by seamount rather than depth. This pattern is clearer for seamounts in the New England seamount chain than in the Corner Rise, indicating that other factors may be confounding this pattern in the Corner Rise. This suggests that while both geography and depth are important, the factor of geography may have a greater impact in determining community structure. 


\section{Faunal assemblages on substrates}

Significant differences were observed for faunal assemblages found on different types of substrate. Natural abiotic substrate (soft sediment, hard substrate, and open water) were seen to be distinct from each other and from anthropogenic abiotic substrates and biotic substrates. The difference of fauna found in the open water is not surprising, as fish dominated this habitat and were rarely observed on any other. The difference in faunal assemblages between soft sediment and hard substrate has been seen in previous seamount studies. The hard substrata on seamounts are dominated by suspension feeders, mainly gorgonians, zoanthids, antipatharian corals, actinarians, pennatulids, hydroids, sponges, ascidians, and crinoids. Echinoderms (including asteroids, ophiuroids, and holothurians) arthropods, polychaetes and molluscs are also found (reviewed in Rogers 1994). A survey of soft sediment on two Pacific seamounts found the soft sediment infauna dominated by polychaetes, peracarid crustaceans, aplacophoran, bivalve and gastropod molluscs, sipunculans, nemerteans, and oligochaetes. The meiofauna are dominated by nematodes and harpacticiod copepods with ostracods, loriciferans, and kinorhynchs also being present, while the epifauna is dominated by xenophyophores (Levin et al. 1986; Levin and Thomas 1988; Levin and Thomas 1989).

The faunal assemblages found on the anthropogenic abiotic substrates, which consisted of items such as bottles and buckets, most resembled other biotic substrates. It may be that these objects provide structure that is similar to other biotic substrates, providing habitat that is off the bottom.

Interestingly, the dead biotic substrates appeared to be intermediate in similarity between the abiotic and living biotic substrates and also distinct from the other living substrates. In a study of the diversity of species associated with two octocorals, Paragorgia arborea and Primnoa resedaeformis, a difference of associates was found on two microhabitats on the corals, the clean, living surface of coral tissue and pockets of detritus and exposed skeleton (Buhl-Mortensen and Mortensen 2005). The associates on 
living coral tissue tended to be fewer and more specialized and obligate symbionts, while those found on the dead skeleton microhabitat tended to be more abundant and facultative symbionts. Many of second microhabitat symbionts were also found on dead parts of other organisms that rise from the seafloor, such as corals and sponges.

The same study also found distinct faunal assemblages found on the different coral hosts, which were significantly correlated with host morphology (Buhl-Mortensen and Mortensen 2005). This is similar to the distinct faunal assemblages observed on different coral families in this study.

\section{Significant Host-Associate Relationships}

Many associates were found to occur on a variety of habitats. There have been anecdotal accounts of potential symbiotic relationships. In order to try to assess significant host-associate relationships, seven species were identified that occurred more than $50 \%$ of the time on the same host, with at least 20 observations. Of these, the Hydroid sp. 2 was observed on a variety of hosts, but most often on dead parts of the host and therefore is probably not an obligate relationship. The ophiuroid Ophioplinthaca abyssalis was also observed on a variety of other hosts, but it was predominantly observed on one coral host, Candidella imbricata. Although not an obligate symbiont, it appears to have a strong preference for this host. An octopus egg case was also observed on a variety of hosts, but only corals.

The remaining four associates, two decapod crustaceans and two ophiuroids, showed a more specific association with coral hosts. It has been noted anecdotally that deep-water corals often serve as hosts for mobile crustacean fauna and ophiuroids and that the decapod crustaceans found on deep-water corals are dominated by anomuran and palaemonid shrimp (Buhl-Mortensen and Mortensen 2004a; Buhl-Mortensen and Mortensen 2004b). The shrimp Bathypalaemonella serratipalma was observed only on the corals Chrysogorgia spp. and Iridogorgia spp., the galatheid Uroptychus sp. 2 was observed only on the antipatharian Parantipathes sp., the ophiuroid Asteroschema 
clavigera was observed on the Paragorgia spp. and Paramuricea spp., and the ophiuroid Ophiocreas oedipus was observed only on the coral Metallogorgia melanotrichos.

Several symbiotic shrimps have been reported in a review of symbiosis in deepwater corals (Buhl-Mortensen and Mortensen 2004b). In the Atlantic Ocean, the shrimps Pandalus propinquus and Caridion gorgoni are reported to be facultative commensals on the deep-water corals Paragorgia arborea and Lophelia pertusa, and the shrimp Synalpheus townsendi is reported to be a facultative commensal on Oculina reefs. In the Pacific Ocean, the shrimp Periclimenes franklini is reported to be a symbiont of deepwater corals in the Coral Sea. P. propinquus is thought to be a facultative symbiont of the corals. The exact relationship is unknown, however it was suggested that both may benefit from this relationship, the shrimp gaining protection for the coral while cleaning the coral surface from sedimenting particles (Buhl-Mortensen and Mortensen 2004a). B. serratipalma may share a similar relationship with the corals it was observed on. Most individuals also appeared to be gravid females, which was confirmed with several collected specimens.

Two anomuran decapods were reported to be associated with deep-water corals. In the Northwest Atlantic, Eumunida pictus occurs on the corals Lophelia pertusa and Primnoa resedaeformis and in the Northeast Atlantic Munidopsis serricornis is a symbiont that seems to be obligate to cnidarians, mainly L. pertusa (Buhl-Mortensen and Mortensen 2004a; Buhl-Mortensen and Mortensen 2004b). Uroptychus sp. 2 was found only on the antipatharian Parantipathes sp. It seems to have a very specific relationship with that host coral. Other chirostylid crabs were also observed on other coral hosts, but were different species. Uropthychus nitidus was observed on the coral Acanella sp. and Uroptychus sp. 1 was observed on an antipatharian identified as either Taxipathes sp. or Sibopathes sp. Uroptychus sp. 3 was observed on the coral Chrysogorgia sp. 


\section{Conclusions}

This study characterized the communities found on seamounts, identify any pattern of relatedness between those communities and identify the factors that are important for structuring those patterns. This study found distinct seamount communities structured by region and depth. The communities in the New England seamount chain were distinct from those in the Corner Rise seamounts. There were also four distinct populations separated by depth, with breaks at $1300 \mathrm{~m}, 2300 \mathrm{~m}$, and $2600 \mathrm{~m}$. However, although depth is an important structuring factor for these communities, they were more similar based on seamount than depth indicating that geography may be a more significant factor than depth.

This study also quantified the relationships between host and associate fauna. Seven taxa were identified to occur at least $50 \%$ or more on the same host and observed at least 20 times or more. These significant relationships varied in degrees from $60 \%$ occurrence to $100 \%$ occurrence, indicating different levels of specificity. Substrate availability was also seen to be an important factor structuring seamount communities, however, the significance of this factor was lost when coarser taxonomic identification was applied to the data. This would indicate the importance of identifying taxa to the lowest possible taxonomic level or else these host-associate and substrate patterns my be lost.

These results indicate the unique communities of found at seamounts and that there are distinct communities structured by geography, geology, depth, and host composition. As seamount communities are threatened by increased pressure from deepsea fisheries, it is imperative to consider these factors for future conservation efforts. 


\section{REFERENCES}

Brewin, P. E., K. I. Stocks, and G. Menezes. 2007. A history of seamount research, p. 41-61. In T. J. Pitcher et al. [eds.], Seamounts: Ecology, Fisheries, \& Conservation. Fish and Aquatic Resources Series. Blackwell Publishing.

Buhl-Mortensen, L., and P. B. Mortensen. 2004a. Crustaceans associated with the deep-water gorgonian corals Paragorgia arborea (L., 1758) and Primnoa resedaeformis (Gunn., 1763) Journal of Natural History 38: 1233-1247.

---. 2004b. Symbiosis in deep-water corals. Symbiosis 37: 33-61.

---. 2005. Distribution and diversity of species associated with deep-sea gorgonian corals off Atlantic Canada, p. 849-879. In A. Freiwald and J. M. Roberts [eds.], Coldwater Corals and Ecosystems. Springer-Verlag.

CAIRNS, S. D. 2007. Studies on the western Atlantic Octocorallia (Gorgonacea:

Primnoidae). Part 8: New records of Primnoidae form the New England and Corner Rise Seamounts. Proceedings of the Biological Society of Washington 120: $243-263$.

CARTES, J. E., and F. SARDA. 1993. Zonation of deep-sea decapod fauna in the Catalan Sea (Western Mediterranean). Marine Ecology Progress Series 94: 27-34.

Clark, M. 2001. Are deepwater fisheries sustainable? -- the example of orange roughy (Hoplostethus altanticus) in New Zealand. Fisheries Research 51: 123-135.

Clarke, K. R., and R. N. Gorley. 2006. PRIMER v6: User manual/tutorial. PRIMERE.

Clarke, K. R., and R. M. WARWICK. 2001. Change in Marine Communities: An Approach to Statistical Analysis and Interpretation, 2nd ed. PRIMER-E.

DunCAN, R. A. 1984. Age progressive volcanism in the New England seamounts and the opening of the central Atlantic ocean. Journal of Geophysical Research 89: 99809990.

EZER, T. 1994. On the interaction between the Gulf Stream and the New England Seamount Chain. Journal of Physical Oceanography 24: 191-204.

Fock, H., F. Uiblen, F. Koster, and H. vON Westernhagen. 2002. Biodiversity and species-environment relationships of the demersal fish assemblage at the Great Meteor Seamount (subtropical NE Atlantic), sampled by different trawls. Marine Biology 141: 185-199.

Gage, J. D., and P. A. Tyler. 1991. Deep-sea biology: a natural history of organisms at the deep-sea floor. Cambridge University Press, Cambridge, New York etc: 1504.

Genin, A., and G. W. BoEHLERT. 1985. Dynamics of temperature and chlorophyll structures above a seamount: an oceanic experiment. Journal of Marine Research 43: 907-924. 
Genin, A., P. K. Dayton, P. F. Lonsdale, and F. N. Spiess. 1986. Corals on seamount peaks provide evidence of current acceleration over deep-sea topography. Nature 322: 59-61.

Gontz, A. M., D. Scheirer, and L. WATLING. in prep/press. Wave-cut features on the Corner Rise Seamount Complex: Marine Geology.

Haedrich, R. L., G. T. Rowe, and P. T. Polloni. 1980. The megabenthic fauna in the deep sea south of New England, USA. Marine Biology 57: 165-179.

Hamilton, E. L. 1956. Sunken islands of the Mid-Pacific Mountains. Geological Society of America Memoir 64.

Hoff, G. R., and B. Stevens. 2005. Faunal Assemblage Structure on the Patton Seamount (Gulf of Alaska, USA). Alaska Fishery Research Bulletin 11: 27-36.

Howell, K. L., D. S. M. Billett, and P. A. Tyler. 2002. Depth-related distribution and abundance of seastars (Echinodermata : Asteroidea) in the Porcupine Seabight and Porcupine Abyssal Plain, NE Atlantic. Deep-Sea Research I 49: 1901-1920.

HubBS, C. L. 1959. Initial Discoveries of Fish Faunas on Seamounts and Offshore Banks in the Eastern Pacific. Pacific Science 13: 311-316.

KAISER, M. J. 1998. Significance of Bottom-Fishing Disuturbance. Conservation Biology 12: $1230-1235$.

Kaufmann, R. S., W. W. Wakefield, and A. Genin. 1989. Distribution of epibenthic megafauna and lebensspuren on two central North Pacific seamounts. Deep Sea Research 36: 1863-1896.

Kitchingman, A., S. Lai, T. Morato, and D. Pauly. 2007. How many seamounts are there and where are they located?, p. 26-40. In T. J. Pitcher et al. [eds.], Seamounts: Ecology, Fisheries, \& Conservation. Fish and Aquatic Resources Series. Blackwell Publishing.

Koslow, J. A., G. W. Boehlert, J. D. M. Gordon, R. L. Haedrich, P. Lorance, and N. PARIN. 2000. Continental slope and deep-sea fisheries: implications for a fragile ecosystem. ICES Journal of Marine Science 57: 548-557.

Koslow, J. A., K. Gowlett-Holmes, J. K. Lowry, G. C. B. Poore, and A. Willams. 2001. Seamount benthic macrofauna off southern Tasmania: community structure and impacts of trawling Marine Ecology Progress Series 213: 111-125.

Levin, L. A., D. J. DeMaster, L. D. McCann, and C. L. Thomas. 1986. Effects of giant protozoans (class: Xenophyophorea) on deep-seamount benthos. Marine Ecology Progress Series 29: 99-104.

LEVIN, L. A., and C. L. Thomas. 1988. The ecology of xenophyophores (Protista) on eastern Pacific seamounts. Deep-Sea Research 12.

---. 1989. The influence of hydrodynamic regime on infaunal assemblages inhabiting carbonate sediments on central Pacific Seamounts. Deep-Sea Research 36: $1897-$ 1915.

LoPHUKIN, A. S. 1986. Distribution of ATP concentration above seamounts in the Atlantic ocean. Oceanology 26: 361-365.

MetaXAs, A., and J. DAVIS. 2005. Megafauna associated with assemblages of deepwater gorgonian corals in Northeast Channel, off Nova Scotia, Canada. Journal of the Marine Biological Association of the United Kingdom 85: 1381-1390. 
Moore, J. A. and others 2003. Biodiversity of Bear Seamount, New England Seamount chain: Results of exploratory trawling. Journal of Northwest Atlantic Fishery Science 31: 363-372.

Mortensen, P. B., and L. Buhl-Mortensen. 2004. Distribution of deep-water gorgonian corals in relation to benthic habitat features in the Northeast Channel (Atlantic Canada). Marine Biology 144: 1223-1238.

MullineauX, L. S., and S. W. Mills. 1997. A test of the larval retention hypothesis in seamount-generated flows. Deep-Sea Research 44: 745-770.

Parin, N. V., A. N. Mironov, and K. N. Nesis. 1997. Biology of the Nazca and Sala y Gomez Submarine Ridges, an Outpost of the Indo-West Pacific Fauna in the Eastern Pacific Ocean: Composition and Distribution of the Fauna, its Communities and History. Advances in Marine Biology 32: 145-242.

Probert, P. K. 1999. Seamounts, sanctuaries, and sustainability: moving towards deepsea conservation. Aquatic Conservation: Marine and Freshwater Ecosystems 9: 601-605.

Probert, P. K., D. G. McKnight, and S. L. Grove. 1997. Benthic invertebrate bycatch from a deep-water trawl fishery, Chatham Rise, New Zealand. Aquatic Conservation: Marine and Freshwater Ecosystems 7: 27-40.

QIU, B. 1994. Determining the mean Gulf Stream and its recirculations through combining hydrographic and altimetric data. Journal of Geophysical Research 99: 951-962.

Rex, M. A. 1981. Community Structure in the Deep-Sea Benthos. Annual Review of Ecology and Systematics: 331-353.

Richardson, P. L. 1980. Anticyclonic eddies generated near Corner Rise seamounts. Journal of Marine Research 38: 673-686.

Richer de Forges, B., J. A. Koslow, and G. C. B. Poore. 2000. Diversity and endemism of the benthic seamount fauna in the southwest Pacific. Nature 405: 944-947.

RodEN, G. I., and B. A. TAFT. 1985. Effect of the Emperor seamount on the mesoscale thermohaline structure during the summer of 1982. Journal of Geophysical Research 90: 839-855.

Roden, G. I., B. A. TAFT, and C. C. EBBESMEYER. 1982. Oceanographic aspects of the Emperor seamounts region. Journal of Geophysical Research 87: 9537-9552.

Rogers, A. D. 1994. The biology of seamounts. Advances in Marine Biology 30: 305350.

Samadi, S., T. Schlacher, and B. R. DE Forges. 2007. Seamount benthos, p. 119-140. In T. J. Pitcher et al. [eds.], Seamounts: Ecology, Fisheries, \& Conservation. Fish and Aquatic Resources Series. Blackwell Publishing.

Shepard, A. N., R. B. Theroux, R. A. CoOper, and J. R. Uzmann. 1986. Ecology of Ceriantharia (Coelenterata, Anthozoa) of the northwestern Atlantic from Cape Hatteras to Nova Scotia. Fishery Bulletin 84: 625-646.

STOCKS, K. I. 2004. Seamount invertebrates: composition and vulnerability to fishing, p. 17-24. In T. Morato and D. Pauly [eds.], Seamounts: Biodiversity and Fisheries. Fisheries Centre, University of British Columbia. 
TAYLOR, G. I. 1917. Motion of solids in fluids when the flow is not irrotational. Proceedings of the Royal Society A 93: 99-113.

Thrush, S. F. and others 1998. Disturbance of the Marine Benthic Habitat by Commercial Fishing: Impacts at the Scale of the Fishery. Ecological Adaptations 8: $866-879$.

Vastano, A. C., D. E. HAgen, and G. J. MCNALly. 1985. Lagrangian observations of surface circulation at the Emperor seamount chain. Journal of Geophysical Research 90: 3325-3331.

Vastano, A. C., and B. A. WARRen. 1976. Perturbations of the Gulf Stream by Atlantis II seamount. Deep-Sea Research 23: 681-694.

WATLING, L. 2007. A review of the genus Iridogorgia (Octocorallia: Chrysogorgiidae) and its relatives, chiefly from the North Atlantic Ocean. Journal of the Marine Biological Association of the United Kingdom 87: 393-402.

WILSON, R. R., JR., and R. S. KAUFMANN. 1987. Seamount biota and biogeography, p. 355-377. In B. H. Keating, P. Fryer, R. Batiza and G. W. Boehlert [eds.], Seamounts, Islands, and Atolls. Geophysical Monograph Series. American Geophysical Union.

Zheng, Y., and J. ARKANI-HAMED. 2002. Rigidity of the Atlantic oceanic lithosphere beneath New England seamounts. Tectonophysics 359: 359-369. 


\section{Chapter 3: Identifying ophiuroids from the North Atlantic seamounts using DNA barcodes}

\section{INTRODUCTION}

There are currently over 1900 known species of ophiuroids making them the largest of the five extant classes of echinoderms (Fell 1960; Tyler 1980). There are some 250 genera that have been described and they are an important marine benthic invertebrate, being diverse and widespread throughout the world. The occupy a range of habitats including the intertidal, estuaries, abyssal plains, and seamounts (Smith et al. 1995). Many ophiuroid species have a large bathymetric range and are distributed from the poles to the tropics (Smith et al. 1995; Tyler 1980).

Ophiuroids were also one of the most speciose and abundant groups observed in recent research cruises to the North Atlantic seamounts with twenty different morphospecies of ophiuroids collected (Figure 1 and Table 1). Within the North Atlantic, approximately 120 species of abyssal ophiuroids have been documented and described (Paterson 1985). In order to characterize the ophiuroid fauna collected from the seamounts, a barcoding approach was taken to try to identify morphospecies to the lowest taxonomic level possible. This was also done to confirm that individuals of four target morphospecies (Asteroschema clavigera Verrill, 1894, Ophiocreas oedipus Lyman, 1879, Ophioplinthaca abyssalis Cherbonnier \& Sibouet, 1972, and Ophioplinthaca chelys Wyville Thomson, 1878) belonged to the same species as defined by the phylogenetic species concept in order to conduct a population genetic study of ophiuroids across the North Atlantic seamounts (Figure 2). The phylogenetic species concept is defined as a monophyletic group that shows a pattern of ancestry and descent (Avise 1994). As there have been reports of cryptic speciation in ophiuroids in the past (Hunter and Halanych 2008; Sponer and Roy 2002), this is a key preliminary step to ensure the proper analyses. 


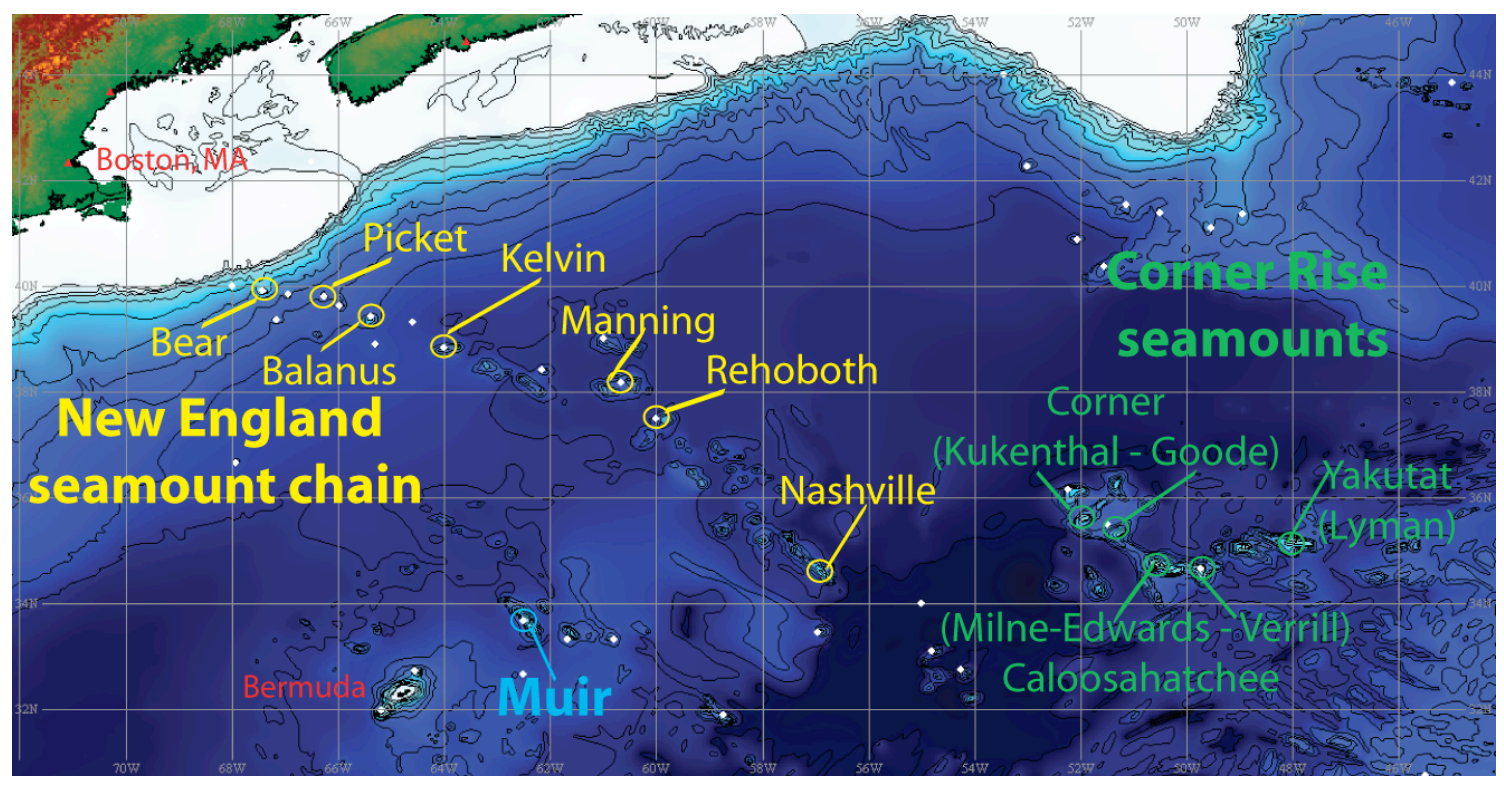

Figure 1. Map of sampled seamounts in the North Atlantic. The New England seamount chain is in yellow, the Corner Rise seamounts in green, and Muir seamount is in blue.

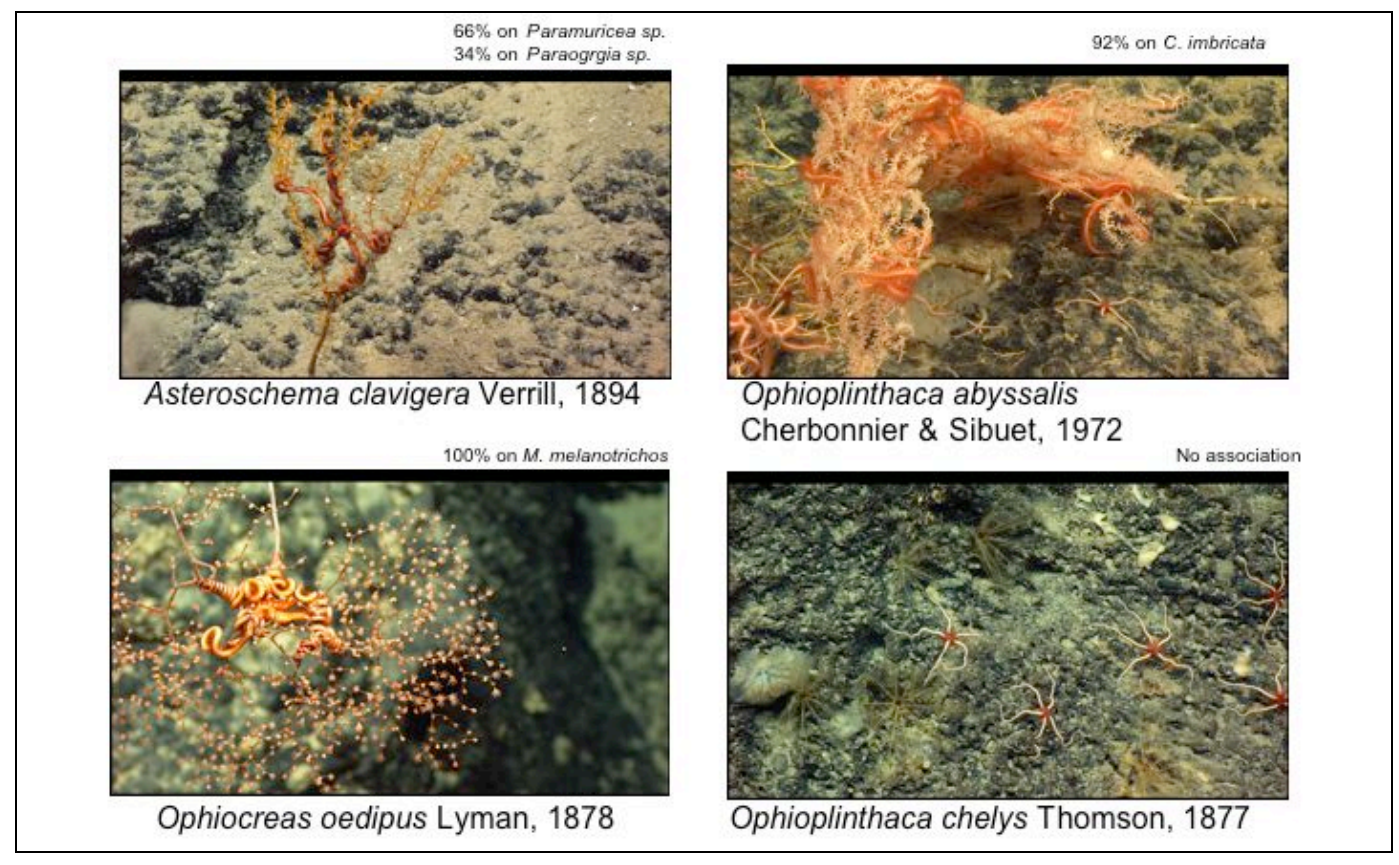

Figure 2. Species targeted for population genetic analyses and identification confirmation. The specificity of the relationship to a host is indicated as a percentage of occurence (Chapter 2). 
Table 1. List of all preliminarily identified ophiuroid morphospecies collected from the North Altantic seamounts. NESC $=$ New England seamount chain.

\begin{tabular}{|c|c|c|c|c|}
\hline & Region & & & \\
\hline Morphospecies & $\begin{array}{l}\text { Corner } \\
\text { Rise }\end{array}$ & NESC & Muir & Total \\
\hline Asteronyx & 23 & 3 & 4 & 30 \\
\hline Asteroschema & 11 & 94 & 22 & 127 \\
\hline Astroporpa & & 3 & & 3 \\
\hline Ophiacantha & 41 & 304 & & 345 \\
\hline Ophiocreas & 9 & 16 & & 25 \\
\hline Ophiuroid sp. 1 & 1 & 23 & & 24 \\
\hline Ophiuroid sp. 2 & 1 & 79 & 16 & 96 \\
\hline Ophiuroid sp. 3 & & 18 & & 18 \\
\hline Ophiuroid sp. 4 & & 2 & & 2 \\
\hline Ophiuroid sp. 6 & 1 & 46 & 47 & 94 \\
\hline Ophiuroid sp. 7 & 7 & & 7 & 14 \\
\hline Ophiuroid sp. 8 & & 1 & 5 & 6 \\
\hline Ophiuroid sp. 9 & & 1 & 1 & 2 \\
\hline Ophiuroid sp. 10 & 452 & 127 & & 579 \\
\hline Ophiuroid sp. 11 & & 8 & & 8 \\
\hline Ophiuroid sp. 13 & 2 & 2 & & 4 \\
\hline Ophiuroid sp. 14 & 14 & 65 & & 79 \\
\hline Ophiuroid sp. 15 & 1 & & & 1 \\
\hline Ophiuroid sp. 16 & 5 & 31 & & 36 \\
\hline Ophiuroid sp. 17 & 1 & & & 1 \\
\hline Total & 569 & 823 & 102 & 1494 \\
\hline
\end{tabular}

There has been a long history of using DNA sequence diversity to discriminate species (Ward et al. 2005). Recently, it has been proposed that the mitochondrial DNA gene cytochrome oxidase subunit I (COI) be used as a "barcode" to serve as a global bioidentification system of animals (Hebert et al. 2003). This gene may not be the most 
appropriate for all taxa, however, as some have been shown to be too variable, as in some copepods (Desalle et al. 2005), or not variable enough, as for some deep-sea octocorals (France and Hoover 2002).

COI has been a particularly difficult gene to work with within ophiuroids (Sponer et al. 2001). On the other hand, the mitochondrial gene $16 \mathrm{~S}$ has successfully been used to identify cryptic species in several cases (Hunter and Halanych 2008; Sponer and Roy 2002). We evaluate the use of $16 \mathrm{~S}$ as a molecular marker to discern species within the class Ophiuroidea in order to confirm preliminary morphospecies identifications from ophiuroids collected from the North Atlantic seamounts.

\section{MATERIALS AND METHODS}

\section{Sample collection}

Samples of ophiuroids were collected from seamount populations in the North Atlantic (Table 1). Most populations were sampled during the 2005 Deep Atlantic Stepping Stones research expedition aboard the $R / V$ Ron Brown using the ROV Hercules. The ROV Hercules was equipped with a Kraft Predator force-reflecting manipulator arm, an ISE Magnum manipulator arm, various adaptors for the manipulator arms for use during collection, insulated bioboxes for sample collection, and two suction pumps. The manipulator arm was modified with cutting blades to aid in the collection of corals and other invertebrates.

During the Deep Atlantic Stepping Stones research cruise, the biological samples were processed immediately after recovery of the ROV Hercules. The ophiuroids were first sorted into preliminary morphospecies. Most morphospecies were frozen whole for later genetic analysis except for four target species that were to be used for later population genetic studies, Asteroschema clavigera Verrill, 1894, Ophiocreas oedipus 
Lyman, 1879, Ophioplinthaca abyssalis Cherbonnier \& Sibouet, 1972, and Ophioplinthaca chelys Wyville Thomson, 1878. Arms of individuals of A. clavigera and O. oedipus were subsampled and frozen for later genetic analysis while the remaining whole individuals were preserved in (formalin, transferred to ethanol, or just $70 \%$ ethanol?). Individuals of $O$. abyssalis and $O$. chelys were frozen whole for later genetic analysis. Voucher specimens mf morphotypes were preserved in $70 \%$ ethanol for later identification based on morphological characters.

Our samples were supplemented by collections made in 2003 during research cruise AT11-7 aboard the $R / V$ Atlantis using the DSV Alvin. These samples contributed additional ophiuroids from Manning seamount and provided all of the ophiuroids from Muir seamount. Similar to the protocol used in 2005, after each dive, samples were sorted into preliminary morphospecies and then frozen for later genetic analysis. Voucher specimens were preserved in $70 \%$ ethanol.

Additional ophiuroid samples preserved in $70 \%$ ethanol from Bear seamount were provided by Dr. Jon Moore from research cruise DE 02-06 in 2002 aboard the $R / V$ Delaware II. Samples from Picket seamount were provided by Dr. Rhian Waller from research cruise AT12-1 aboard the $R / V$ Atlantis and collected using the DSV Alvin. They were processed and frozen for storage similar to the samples from cruise AT11-7. Additional ophiuroid samples were also provided by the United States National Museum and Ken Halanych and Rebecca Belcher Hunter from Auburn University.

\section{DNA extraction, polymerase chain reaction, and sequencing}

DNA was extracted from representatives of every morphotype collected. Particular interest was given to the four target species mentioned above. A variety of extraction protocols were used. The majority of ophiuroids were extracted using a 5\% Chelex extraction protocol as described in Sponer and Roy, 2002 (Sponer and Roy 2002; Walsh et al. 1991). A small piece of tissue ranging from 1-3 $\mathrm{mm}$ of an arm was minced 
and then added to $500 \mu 1$ of $5 \%$ Chelex (Bio-Rad Hercules, CA) solution. This was heated for $3 \mathrm{~h}$ at $65^{\circ} \mathrm{C}$ with the issue completely submerged within the chelex beads. The solution was then vortexed and heated to $95^{\circ}-100^{\circ} \mathrm{C}$ for 10 minutes and then centrifuged at $13,500 \times \mathrm{g}$ for 10 minutes. Ethanol-preserved samples were processed in the same way with the addition of an initial rinse of the tissue in water. The extractions were stored in $4^{\circ} \mathrm{C}$ for short-term storage and $-20^{\circ} \mathrm{C}$ for long-term storage. Additionally, a modified Chelex protocol was used for some individuals, which extended the incubation to overnight as well as a DNA extraction method utilizing a cetyltrimethylammonium bromide (CTAB)-based protocol including a phenol-chloroform cleaning step.

A fragment of the mitochondrial DNA gene 16S rRNA (16S) was amplified with the primers 16SarL (5'-CGCCTGTTTATCAAAAACAT-3') and 16SbrH (5'CCGGTCTGAACTCAGATCACGT-3') (Palumbi et al. 1991). These primers worked consistently for all four species and were used for sequencing gene fragments of varying sizes depending on the species (Table 1). Most fragments were amplified in $25 \mu 1$ PCR reactions containing 1X PCR buffer (Promega), $2.5 \mathrm{mM} \mathrm{MgCL} 2,1.0 \mathrm{mM}$ of each dNTP, $0.5 \mu \mathrm{M}$ of each primer, $0.5 \mathrm{M}$ Betaine, $1-2 \mu \mathrm{l}$ of extracted template, and $0.2 \mathrm{U}$ of Taq polymerase (Promega). Some fragments were amplified using 1X PCR Buffer (Eppendorf), $2.5 \mathrm{mM} \mathrm{MgCL} 2,1.0 \mathrm{mM}$ of each dNTP, $0.5 \mu \mathrm{M}$ of each primer, $1 \mathrm{X}$ TaqMaster PCR Enhancer (Eppendorf), 1-2 $\mu$ l of extracted template, and $0.2 \mathrm{U}$ of Taq polymerase (Eppendorf). PCR was performed under the following conditions: initial denaturation for $2 \mathrm{~min}$ at $94^{\circ} \mathrm{C}$, followed by 30 cycles of denaturation at $94^{\circ} \mathrm{C}$ for $1 \mathrm{~min}$, 30 cycles of annealing at $45^{\circ} \mathrm{C}$ for $1 \mathrm{~min}$, and 30 cycles of extension at $72^{\circ} \mathrm{C}$ for $45 \mathrm{sec}$, followed by a final extension phase of $72^{\circ} \mathrm{C}$ for $7 \mathrm{~min}$.

Successfully amplified PCR products of $16 \mathrm{~S}$ were directly purified using either the QIAquick PCR Purification kit (Qiagen) or the SV Gel and PCR Cleanup Kit (Promega) and eluted into a final $30 \mu \mathrm{l}$ volume. Purified PCR products were then used in one-eighth format sequencing reactions using Big Dye terminators (version 3, PerkinElmer) in 96-well plates and purified by isopropanol precipitation before sequencing on 
an ABI 3700 Capillary Sequencer. PCR products were sequenced bi-directionally except for a few cases where only one direction was obtained.

\section{S ophiuroid species profile}

In order to get the most coverage possible of all ophiuroid families, our own sequences were supplemented with 48 additional sequences from GenBank in July 2008. One to five representative $16 \mathrm{~S}$ sequences of every available ophiuroid species was included, resulting in a total of $35416 \mathrm{~S}$ sequences.

\section{Data analysis}

Sequences were edited in Sequencher 4.8 (Gene Codes Corporation Inc.). All sequences, including the ones from GenBank were aligned using MAFFT v6.527, an alignment algorithm based on fast Fourier transformation that was developed specifically for large datasets and which is claimed to be more accurate than other automated alignment packages (Katoh et al. 2002). MAFFT was left at the default settings and the iterative refinement chosen was the option 'E-INS-I'. Alignments were viewed by eye in MacClade 4.08 (Maddison and Maddison 2000) and trimmed to an alignment width of $710 \mathrm{bp}$.

Nucleotide-sequence divergences were calculated using the Kimura two parameter (K2P) distance model (Kimura 1980). The K2P model was chosen because it makes fewer assumptions about the nature of sequence change than more heavily parameterized models, which is important when transition rules governing nucleotide substitutions vary among taxa, and it also provides a conservative estimate of long branches as it underestimates the number of multiple hits in comparison to more complex models (Barrett and Hebert 2005; Hebert et al. 2003; Nei and Kumar 2000). We then 
employed a neighbor-joining analysis with MEGA version 4.0 with bootstraps of 1000 (Tamura et al. 2007).

\section{RESULTS}

An examination of the neighbour-joining tree revealed evidence for the clustering of taxonomically allied species (Figure 3). Of the 8 known genera that possessed multiple species (ranging from 2 to 14 species), 7 formed cohesive groups. The only unexpected placement was a sequence of Ophiomyxa flaccida, which was placed within the clade of the genus Ophioderma. O. flaccida is in the family Ophiomyxidae which is thought to be a sister group to all other families within the order Ophiurida. Also, there was a moderate association at the family level, with 10 out of 12 known families forming cohesive assemblages. Another unexpected result was the placement of Asteronyx sp. within the clade comprising the family Gorgonocephalidae, rather than forming another clade since it is in the family Asteronychidae.

The average K2P distance of individuals within species was $3.41 \%$ compared to $10.88 \%$ for species within genera (Table 2 and Table 3). The K2P distances for individuals within a species included Ophiothrix fragilis (6.9\%), Amphipholis squamtata (15.4\%), and Ophiura albida (14.4\%). Both O. fragilis, and A. squamata have both been shown to have cryptic speciation (Baric and Sturmbauer 1999; Sponer et al. 2001). Ophiuroids typically have been shown to have 3\% sequence divergence between species (Roy and Sponer 2002). Excluding these three species, which have potential cryptic speciation, the average genetic divergence is $0.467 \%$. There is overall $\sim 23$ times more variation among congeneric species than conspecific indivudals. Mean divergence among families increases to $15.23 \%$ and among species within the ophiuroid orders Ophiurida and Euryalida it is $32.9 \%$ and $8.2 \%$ respectively (Table 4 and Table 5). 
Table 2. Mean of intraspecific sequence divergences for 12 ophiuroid species, using Kimura's two parameter model.

\begin{tabular}{|c|c|c|c|}
\hline \multirow[b]{2}{*}{ Species } & \multirow[b]{2}{*}{ Number of individuals } & \multicolumn{2}{|l|}{ Mean Percent } \\
\hline & & Divergence & S.E. \\
\hline Macrophiothrix nereidi & 2 & 0 & $\overline{0}$ \\
\hline Ophionereis reticulata & 2 & 0.6 & 0.003 \\
\hline Ophiopholis aculeata & 2 & 0.2 & 0.002 \\
\hline Ophiothrix fragilis & 4 & 6.9 & 0.01 \\
\hline Amphipholis squamata & 7 & 15.4 & 0.012 \\
\hline Ophiura albida & 2 & 14.4 & 0.018 \\
\hline Ophiothrix trilineata & 2 & 0 & 0 \\
\hline Ophiocreas oedipus & 14 & 0.1 & 0.001 \\
\hline Ophioplinthaca abyssalis & 62 & 1.1 & 0.002 \\
\hline Ophioplinthaca chelys & 43 & 1.5 & 0.002 \\
\hline Ophioplinthaca bidentata & 2 & 0.4 & 0.003 \\
\hline \multirow[t]{3}{*}{ Asteroschema clavigera } & 35 & 0.3 & 0.001 \\
\hline & Average & 3.41 & \\
\hline & Range & $0-15.4$ & \\
\hline \multicolumn{2}{|c|}{$\begin{array}{l}\text { Average (excluding O. fragilis, A. squamata, and } O \text {. } \\
\text { albida) }\end{array}$} & 0.467 & \\
\hline \multicolumn{2}{|c|}{$\begin{array}{l}\text { Range (excluding O. fragilis, A. squamata, and O. } \\
\text { albida) }\end{array}$} & $0-1.5$ & \\
\hline
\end{tabular}




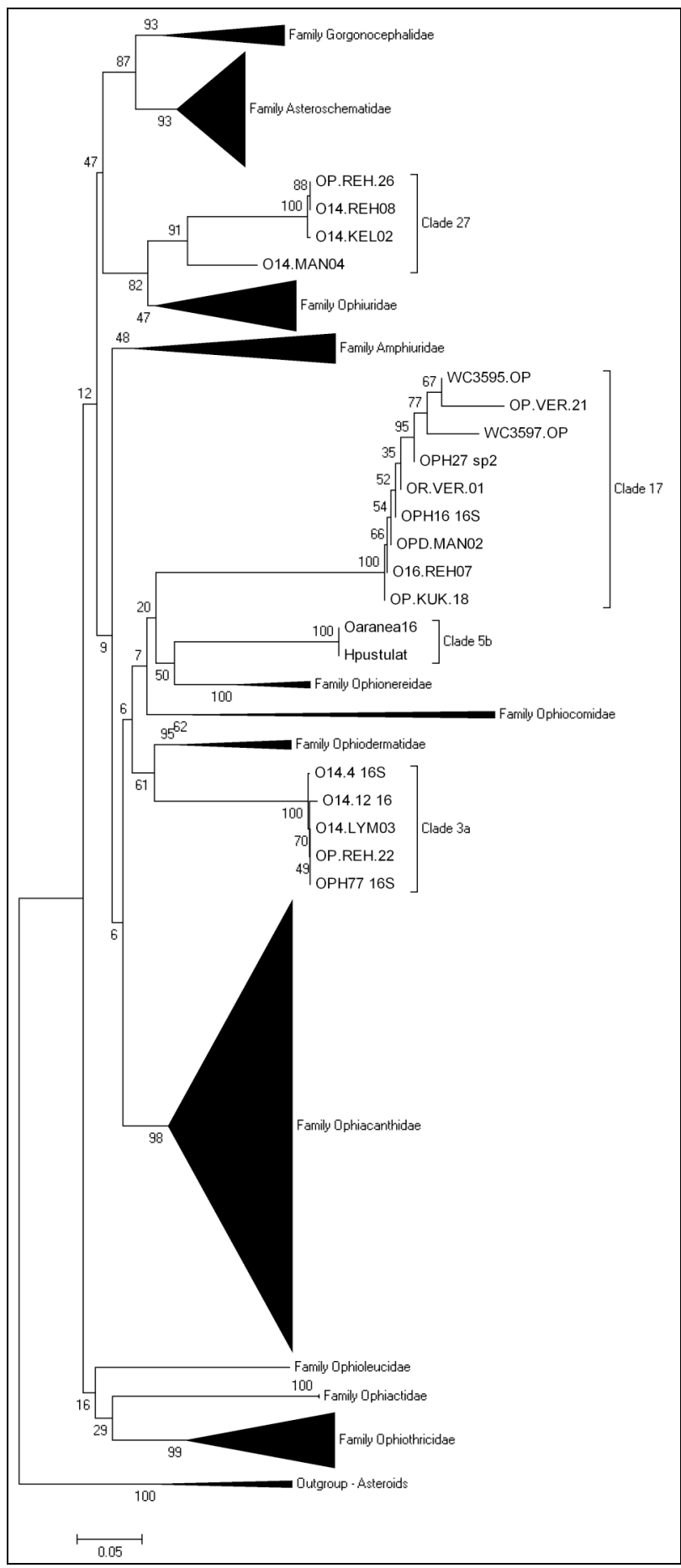

Figure 3. Neighbour-joining tree of 354 16S sequences from ophiuroids species using K2P distances. Families have been collapsed and remaining clades are those species not identified. 
Table 3. Mean of interspecific sequence divergences for 7 ophiuroid genera, using Kimura's two parameter model.

\begin{tabular}{|c|c|c|c|c|c|}
\hline Genus & $\begin{array}{l}\text { Number of } \\
\text { Species }\end{array}$ & & $\begin{array}{l}\text { Mean Percent } \\
\text { Divergence }\end{array}$ & & S.E. \\
\hline Amphipholis & & 2 & & 17.8 & 0.013 \\
\hline Macrophrithrix & & 14 & & 17 & 0.012 \\
\hline Ophioderma & & 3 & & 13.7 & 0.018 \\
\hline Ophiothrix & & 3 & & 5.1 & 0.007 \\
\hline Amphiura & & 2 & & 21.3 & 0.023 \\
\hline Ophiura & & 2 & & 9.5 & 0.011 \\
\hline \multirow[t]{3}{*}{ Ophioplinthaca } & & 2 & & 8.2 & 0.009 \\
\hline & Average & & \multicolumn{3}{|c|}{13.22857143} \\
\hline & Range & & \multicolumn{3}{|c|}{$5.1-21.3$} \\
\hline
\end{tabular}

Table 4. Mean sequence divergences between genera for 6 ophiuroid families, using Kimura's two parameter model.

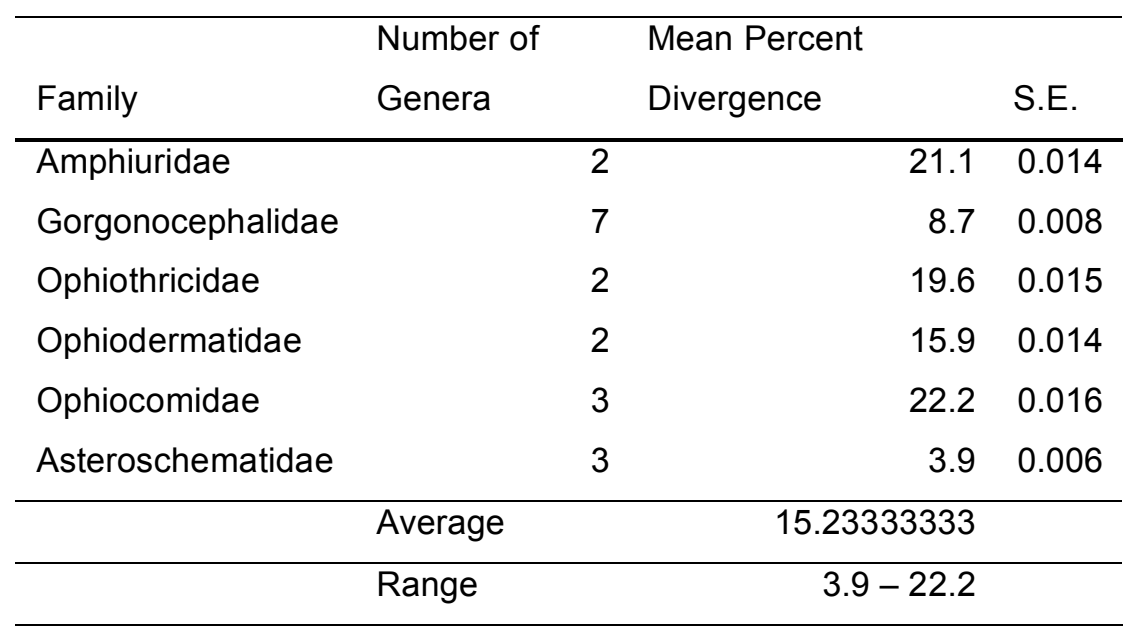


Table 5. Mean sequence divergences between families for 2 ophiuroid orders, using Kimura's two parameter model.

\begin{tabular}{lllrl}
\hline & Number of & & Mean Percent \\
Order & Families & & Divergence & \\
\hline Ophiurida & 8 & & 32.9 & 0.019 \\
Euryalida & 2 & 8.2 & 0.007 \\
\hline
\end{tabular}

The four target species of ophiuroids were identified and formed 4 separate clades. The preliminary identifications were not correct for all four species. Within the O. oedipus clade, four individuals were preliminarily identified as $A$. clavigera. Also, one individual of $A$. clavigera, ANX4 was neither $O$. oedipus or $A$. clavigera (Figure 4). Within the identified clades, however, $O$. oedipus had a mean sequence divergence of $0.1 \%$ and $A$. clavigera had a mean percent divergence of $0.3 \%$. Ophiuroids preliminarily identified as $O$. abyssalis also did not form a single clade. The $O$. abyssalis clade had a mean sequence divergence of $1.1 \%$ and consisted mostly of individuals identified as $O$. abyssalis, but there were also one individual identified as $O$. chelys and one individual identified as ophiuroid sp. 2 (Figure 5). The individuals identified as $O$. chelys also did not cluster together, but instead were scattered among the clades of the tree. Those individuals within the $O$. chelys clade were mostly those identified as $O$. chelys, except for one individuals identified as ophiuroid sp. 14 and another identified as ophiuroid sp. 7 (Figure 6). 


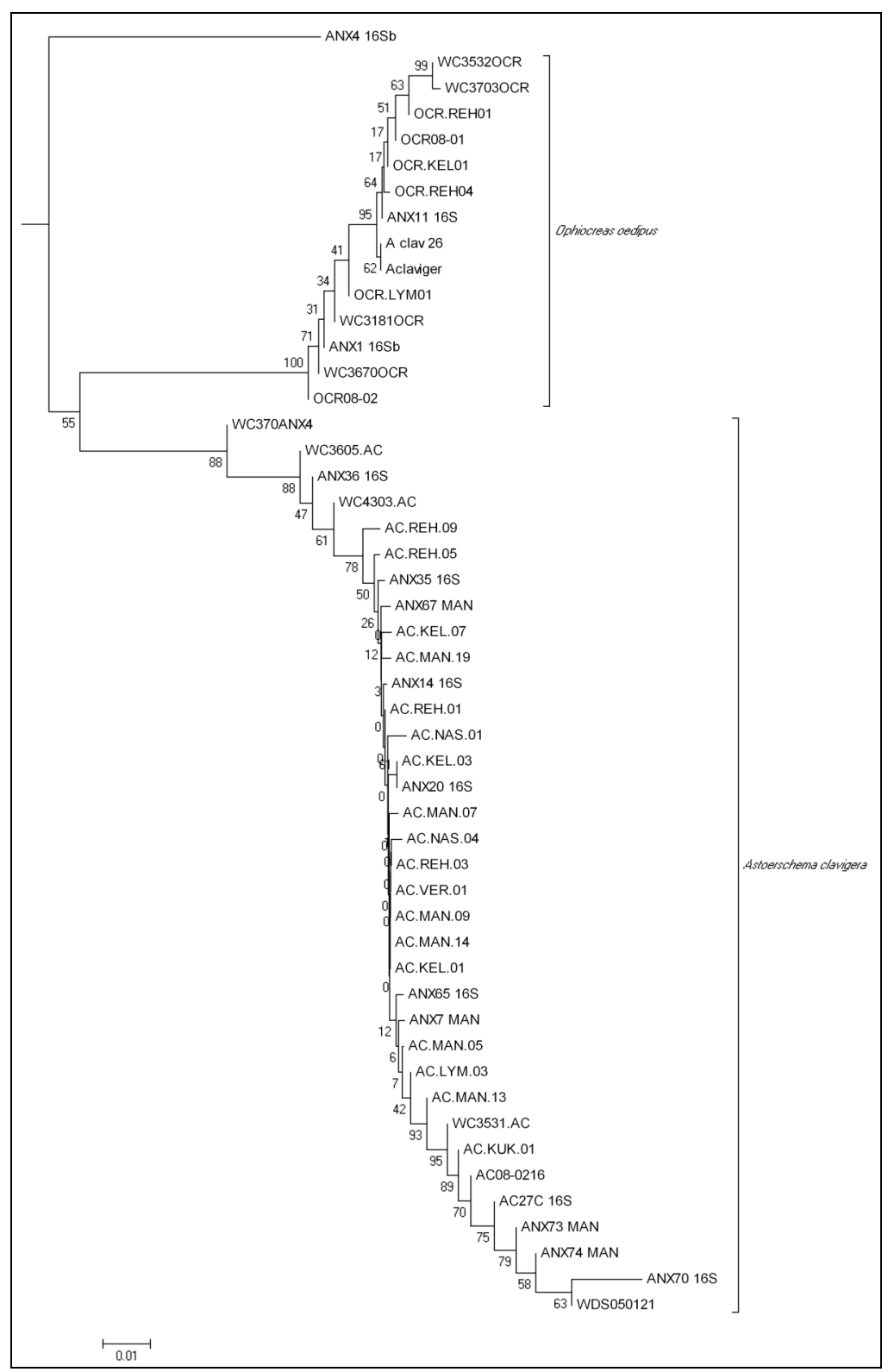

Figure 4. Family Asteroschematidae clade from a neighbour-joining analysis of 354 ophiuroid sequences. 


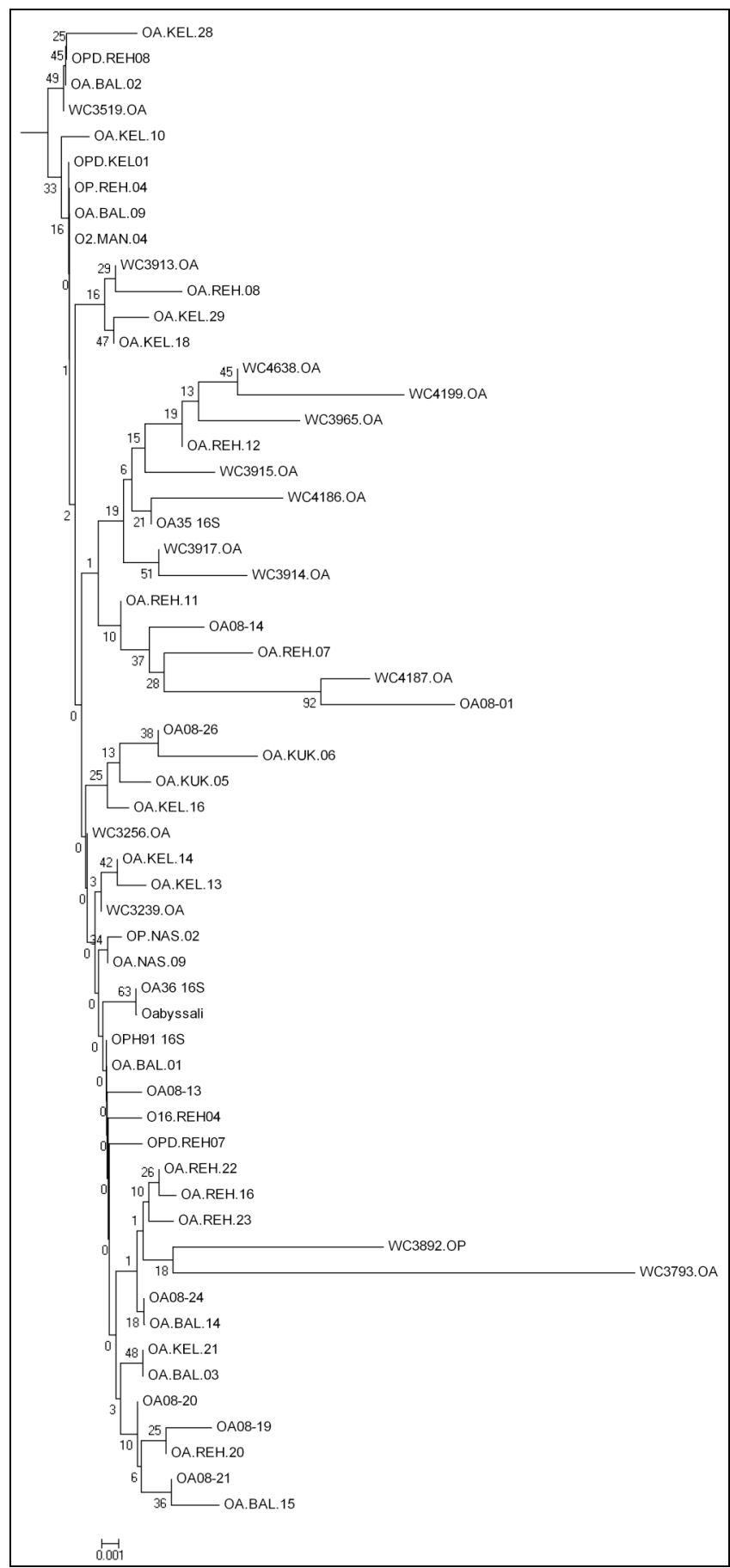

Figure 5. Clade of $O$. abyssalis from a neighbour-joining analysis of 354 ophiuroid sequences. 


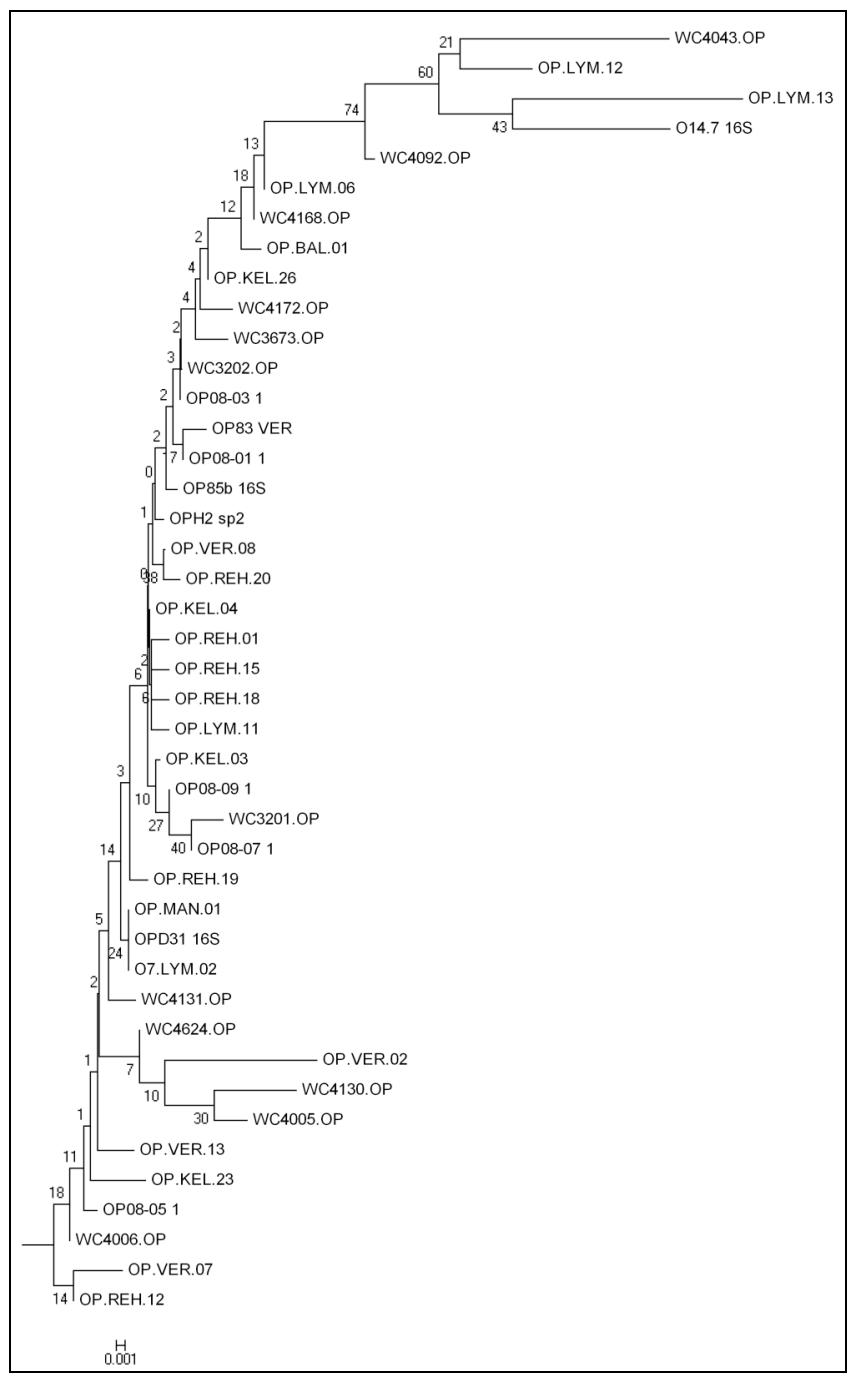

Figure 6. Clade of $\boldsymbol{O}$. chelys from a neighbour-joining analysis of 354 ophiuroid sequences.

The other ophiuroids collected from the North Atlantic seamounts formed into several clades that clustered with known samples. Based on the mean sequence divergence between ophiuroid families established in this study, these clades could be clustered with the known families based on the mean sequence divergence between clades Gorgonocephalidae, Ophiocomidae, Ophiacanthidae, Amphiuridae, and Ophiuridae. Clade 17 could not be placed and is thought to belong to a family not represented in this dataset (Table 6). Eight clades fell within the Family Ophiacanthidae. 
Table 6. Pairwise genetic distances between clades, using K2P.

\begin{tabular}{|c|c|c|c|c|c|c|c|c|c|c|c|c|c|c|c|}
\hline & $\begin{array}{c}\text { O. } \\
\text { oedipus }\end{array}$ & ANX4 & $\begin{array}{c}\text { A. } \\
\text { clavigera }\end{array}$ & Gorgonocephalidae & $\begin{array}{c}\text { Clade } \\
3 a\end{array}$ & Ophiocomidae & Ophiodermatidae & Ophionereididae & $\begin{array}{c}\text { Clade } \\
5 \mathrm{~b}\end{array}$ & $\begin{array}{c}\text { Clade } \\
6\end{array}$ & $\begin{array}{c}\text { O. } \\
\text { abyssalis }\end{array}$ & $\begin{array}{c}\text { Clade } \\
8\end{array}$ & $\begin{array}{c}\text { Clade } \\
9\end{array}$ & $\begin{array}{c}\text { Clade } \\
10\end{array}$ & $\begin{array}{c}\text { Clade } \\
11\end{array}$ \\
\hline O. oedipus & - & & & & & & & & & & & & & & \\
\hline ANX4 & 0.11 & - & & & & & & & & & & & & & \\
\hline A. clavigera & 0.082 & 0.103 & - & & & & & & & & & & & & \\
\hline Gorgonocephalidae & 0.207 & 0.189 & 0.196 & - & & & & & & & & & & & \\
\hline Clade $3 a$ & 0.283 & 0.275 & 0.272 & 0.302 & - & & & & & & & & & & \\
\hline Ophiocomidae & 0.378 & 0.398 & 0.392 & 0.419 & 0.349 & - & & & & & & & & & \\
\hline Ophiodermatidae & 0.287 & 0.297 & 0.312 & 0.295 & 0.233 & 0.371 & - & & & & & & & & \\
\hline Ophionereididae & 0.3 & 0.298 & 0.321 & 0.293 & 0.292 & 0.361 & 0.258 & - & & & & & & & \\
\hline Clade $5 b$ & 0.291 & 0.285 & 0.293 & 0.295 & 0.283 & 0.381 & 0.27 & 0.226 & - & & & & & & \\
\hline Clade 6 & 0.307 & 0.285 & 0.296 & 0.319 & 0.288 & 0.393 & 0.276 & 0.281 & 0.306 & - & & & & & \\
\hline O. abyssalis & 0.279 & 0.27 & 0.296 & 0.299 & 0.293 & 0.387 & 0.284 & 0.289 & 0.336 & 0.086 & - & & & & \\
\hline Clade 8 & 0.322 & 0.319 & 0.322 & 0.357 & 0.297 & 0.402 & 0.285 & 0.283 & 0.309 & 0.155 & 0.148 & - & & & \\
\hline Clade 9 & 0.296 & 0.309 & 0.304 & 0.334 & 0.306 & 0.403 & 0.293 & 0.275 & 0.335 & 0.119 & 0.128 & 0.06 & - & & \\
\hline Clade 10 & 0.316 & 0.301 & 0.324 & 0.337 & 0.271 & 0.381 & 0.28 & 0.275 & 0.269 & 0.143 & 0.151 & 0.116 & 0.12 & - & \\
\hline Clade 11 & 0.305 & 0.297 & 0.317 & 0.343 & 0.277 & 0.38 & 0.264 & 0.261 & 0.285 & 0.153 & 0.151 & 0.117 & 0.114 & 0.118 & - \\
\hline Clade 12 & 0.318 & 0.296 & 0.325 & 0.351 & 0.314 & 0.401 & 0.296 & 0.301 & 0.331 & 0.177 & 0.163 & 0.138 & 0.143 & 0.153 & 0.097 \\
\hline Clade 13 & 0.305 & 0.306 & 0.335 & 0.34 & 0.281 & 0.418 & 0.26 & 0.294 & 0.288 & 0.168 & 0.186 & 0.172 & 0.191 & 0.159 & 0.164 \\
\hline O. chelys & 0.317 & 0.286 & 0.315 & 0.317 & 0.294 & 0.425 & 0.286 & 0.292 & 0.294 & 0.168 & 0.155 & 0.17 & 0.175 & 0.163 & 0.161 \\
\hline Ophiacanthidae & 0.275 & 0.278 & 0.278 & 0.275 & 0.248 & 0.36 & 0.26 & 0.262 & 0.293 & 0.211 & 0.19 & 0.219 & 0.207 & 0.209 & 0.21 \\
\hline Clade 16 & 0.275 & 0.27 & 0.29 & 0.278 & 0.236 & 0.356 & 0.258 & 0.247 & 0.273 & 0.192 & 0.187 & 0.201 & 0.191 & 0.197 & 0.195 \\
\hline Clade 17 & 0.389 & 0.365 & 0.393 & 0.397 & 0.372 & 0.447 & 0.354 & 0.347 & 0.315 & 0.378 & 0.395 & 0.382 & 0.379 & 0.362 & 0.335 \\
\hline Ophiactidae & 0.307 & 0.292 & 0.314 & 0.346 & 0.322 & 0.391 & 0.353 & 0.307 & 0.333 & 0.364 & 0.359 & 0.358 & 0.367 & 0.359 & 0.351 \\
\hline Ophiothricidae-1 & 0.333 & 0.368 & 0.342 & 0.349 & 0.367 & 0.447 & 0.372 & 0.336 & 0.4 & 0.354 & 0.366 & 0.354 & 0.373 & 0.363 & 0.376 \\
\hline Ophiothricidae-2 & 0.378 & 0.38 & 0.378 & 0.375 & 0.384 & 0.441 & 0.385 & 0.344 & 0.402 & 0.372 & 0.387 & 0.404 & 0.395 & 0.379 & 0.388 \\
\hline Ophiothricidae-3 & 0.429 & 0.413 & 0.429 & 0.411 & 0.375 & 0.471 & 0.421 & 0.373 & 0.455 & 0.391 & 0.387 & 0.428 & 0.392 & 0.383 & 0.405 \\
\hline Ophioleucidae & 0.296 & 0.309 & 0.314 & 0.339 & 0.316 & 0.407 & 0.304 & 0.31 & 0.33 & 0.318 & 0.339 & 0.349 & 0.37 & 0.328 & 0.312 \\
\hline Amphiuridae-1 & 0.294 & 0.317 & 0.314 & 0.308 & 0.282 & 0.395 & 0.286 & 0.278 & 0.275 & 0.31 & 0.295 & 0.299 & 0.319 & 0.303 & 0.291 \\
\hline Amphiuridae-2 & 0.332 & 0.34 & 0.337 & 0.349 & 0.363 & 0.455 & 0.349 & 0.35 & 0.382 & 0.344 & 0.329 & 0.363 & 0.363 & 0.369 & 0.355 \\
\hline Clade 25 & 0.325 & 0.329 & 0.354 & 0.365 & 0.333 & 0.455 & 0.348 & 0.311 & 0.344 & 0.33 & 0.315 & 0.304 & 0.327 & 0.33 & 0.331 \\
\hline Ophiuridae & 0.229 & 0.258 & 0.242 & 0.256 & 0.311 & 0.421 & 0.309 & 0.313 & 0.332 & 0.328 & 0.311 & 0.332 & 0.324 & 0.316 & 0.321 \\
\hline Clade 27 & 0.261 & 0.272 & 0.273 & 0.292 & 0.329 & 0.448 & 0.294 & 0.314 & 0.303 & 0.323 & 0.304 & 0.314 & 0.327 & 0.329 & 0.331 \\
\hline Ophiuridae & 0.285 & 0.264 & 0.272 & 0.3 & 0.338 & 0.459 & 0.318 & 0.33 & 0.343 & 0.322 & 0.322 & 0.324 & 0.324 & 0.345 & 0.331 \\
\hline
\end{tabular}


Table 6. continued

\begin{tabular}{|c|c|c|c|c|c|c|c|c|c|c|c|c|c|c|}
\hline & $\begin{array}{c}\text { Clade } \\
12\end{array}$ & $\begin{array}{c}\text { Clade } \\
13\end{array}$ & $\begin{array}{c}\mathrm{O} . \\
\text { chelys }\end{array}$ & Ophiacanthidae & $\begin{array}{c}\text { Clade } \\
16\end{array}$ & $\begin{array}{c}\text { Clade } \\
17\end{array}$ & Ophiactidae & $\begin{array}{c}\text { Ophiothricidae- } \\
1\end{array}$ & $\begin{array}{c}\text { Ophiothricidae- } \\
2\end{array}$ & $\begin{array}{c}\text { Ophiothricidae- } \\
3\end{array}$ & Ophioleucidae & $\begin{array}{c}\text { Amphiuridae- } \\
1\end{array}$ & $\begin{array}{c}\text { Amphiuridae- } \\
2\end{array}$ & $\begin{array}{c}\text { Clade } \\
25\end{array}$ \\
\hline Clade 12 & - & & & & & & & & & & & & & \\
\hline Clade 13 & 0.213 & - & & & & & & & & & & & & \\
\hline O. chelys & 0.207 & 0.16 & - & & & & & & & & & & & \\
\hline Ophiacanthidae & 0.199 & 0.227 & 0.197 & - & & & & & & & & & & \\
\hline Clade 16 & 0.182 & 0.19 & 0.193 & 0.096 & - & & & & & & & & & \\
\hline Clade 17 & 0.366 & 0.323 & 0.343 & 0.347 & 0.331 & - & & & & & & & & \\
\hline $\begin{array}{l}\text { Ophiactidae } \\
\text { Ophiothricidae- }\end{array}$ & 0.348 & 0.399 & 0.37 & 0.331 & 0.316 & 0.35 & - & & & & & & & \\
\hline 1 & 0.376 & 0.361 & 0.358 & 0.336 & 0.315 & 0.4 & 0.326 & - & & & & & & \\
\hline Ophiothricidae- & 0.399 & 0.409 & 039 & 0.364 & 0342 & 0.403 & 0.327 & 0223 & & & & & & \\
\hline Ophiothricidae- & & & & & & & & & & & & & & \\
\hline 3 & 0.376 & 0.43 & 0.407 & 0.365 & 0.349 & 0.441 & 0.353 & 0.239 & 0.234 & - & & & & \\
\hline Ophioleucidae & 0.365 & 0.31 & 0.335 & 0.326 & 0.313 & 0.336 & 0.318 & 0.325 & 0.341 & 0.395 & - & & & \\
\hline Amphiuridae-1 & 0.324 & 0.283 & 0.314 & 0.288 & 0.272 & 0.329 & 0.316 & 0.359 & 0.381 & 0.399 & 0.317 & - & & \\
\hline Amphiuridae-2 & 0.364 & 0.346 & 0.342 & 0.337 & 0.342 & 0.374 & 0.337 & 0.403 & 0.433 & 0.434 & 0.333 & 0.268 & - & \\
\hline Clade 25 & 0.338 & 0.321 & 0.339 & 0.325 & 0.326 & 0.406 & 0.346 & 0.405 & 0.415 & 0.419 & 0.339 & 0.294 & 0.313 & - \\
\hline Ophiuridae & 0.342 & 0.303 & 0.323 & 0.323 & 0.304 & 0.383 & 0.345 & 0.336 & 0.371 & 0.362 & 0.3 & 0.266 & 0.315 & 0.325 \\
\hline Clade 27 & 0.329 & 0.335 & 0.338 & 0.325 & 0.31 & 0.371 & 0.346 & 0.349 & 0.381 & 0.379 & 0.277 & 0.321 & 0.322 & 0.348 \\
\hline Ophiuridae & 0.349 & 0.312 & 0.332 & 0.304 & 0.286 & 0.405 & 0.39 & 0.369 & 0.439 & 0.409 & 0.308 & 0.305 & 0.344 & 0.32 \\
\hline
\end{tabular}

\section{Table 6. continued}

\begin{tabular}{c|ccc}
\hline & Ophiuridae & Clade 27 & Ophiuridae \\
\hline Ophiuridae & - & & \\
Clade 27 & 0.213 & - & \\
Ophiuridae & 0.216 & 0.231 & -
\end{tabular}




\section{DISCUSSION}

This study provides support that a 16S-based identification system will be effective for identifying ophiuroid species. There is a much lower level of intraspecific sequence variation than interspecific sequence variation. Most of the ophiuroids collected from the North Atlantic seamounts belong to the family Ophiacanthidae, which is not surprising as this family occurs primarily in deep water and many of the genera of this family are only found below the shelf edge (Tyler 1980).

The mitochondrial gene $16 \mathrm{~S}$ appears to be a viable marker for confirming species identity. It identified species and families with moderate success. The neighbour-joining analysis allowed species to cluster together, however it was not enough to delineate the phylogenetic relationships of the organisms. The resulting $16 \mathrm{~S}$ neighbour-joining tree, although clustering species into families with some success, could not characterize the relationships between those clades. It did not isolate or recognize the two main orders within the class Ophiuroidea, the order Euryalida and the order Ophiurida which are sister groups to each other within the subclass Ophiuridea (Smith et al. 1995). This result emphasizes an important point in the use of DNA barcodes. Several papers that have used DNA barcodes have extended beyond simply confirming or identifying species to describe the phylogenetic signal that may be present in an analysis such as neighbourjoining (Desalle et al. 2005; Ward et al. 2005). Depending upon the molecular marker used, DNA barcodes may be adequate to delineate species, but they may not be sensitive enough to describe phylogenetic relationships correctly between species (Desalle et al. 2005). In this case, the neighbour-joining analysis did not reflect the relationships thought to exist within the class Ophiuroidea.

There were examples of this in the unexpected placements of some taxa within certain clades. Asteronyx sp. was placed within the clade for the family Gorgonocephalidae when the genus Asteronyx actually belongs to the family Asteronychidae, a sister group to Gorgonocephalidae (Smith et al. 1995). This taxon was 
not identified to species level however and it is a GenBank sequence. It may be that it was initially misidentified. Another unexpected placement was the clustering of Hemieuryale pustulata with Ophiopsila aranea. Both are from different families and the exact relationship of the family Hemieuryalidae with other families within the order Ophiurida is unknown. These two examples highlight the importance of the correct initial identification of specimens, especially for the use as a DNA barcode. This need has been an important point in the discussion of DNA barcodes (Barrett and Hebert 2005; Hebert et al. 2003).

Previous studies have found it difficult to calibrate a molecular clock in ophiuroids, especially with highly divergent 16S mtDNA sequences making it difficult to align sequences with a common outgroup (Le Gac et al. 2004). However, using an approximate echinoderm mtDNA divergence rate of 3.1\%-3.5\%/my (Lessios et al. 1999; Mccartney et al. 2000), a rough context of the timeframe for the evolution of the ophiuorids can be determined. Based upon the average sequence divergences among species, genera, family, and order, divergence for these groups can be dated approximately to 0.97-1.1 mya for species, 3.77-4.26 mya for genera, 4.35-4.91 mya for families, and 9.4-10.6 mya (Ophiurida) and 2.34-2.64 mya (Euryalida) for orders respectively. Based upon the sequence divergences within the four target species, $A$. clavigera diverged approximately 0.086-0.097 mya, O. oedipus diverged approximately 0.029-0.032 mya, O. abyssalis diverged approximately 0.314-0.355 mya, and $O$. chelys diverged approximately 0.429-0.0483 mya. These divergence times are all far shorter than the formation of the seamounts, the youngest being the Corner Rise seamounts which are thought to have been formed approximately 75 mya (Duncan 1984; Zheng and Arkani-Hamed 2002). This would indicate that the ophiuroids could have diversified on the seamounts, supporting the hypothesis of seamounts as centers of speciation. The ophiuroids are thought to have undergone a similar rapid diversification during the Olenekian (249.7-245 mya) after experiencing an evolutionary bottleneck in the Late Permian (299-251 mya) (Chen and McNamara 2006). 
Our preliminary identifications of the ophiuroids were very poor. No identified morphospecies remained clustered as a species. A total of 22 putative ophiuroid species were found on the seamounts. Within these, the four target species were identified and revealed mistaken preliminary morphospecies identifications. This result emphasizes the importance of confirming the species identity of any organism that may be used for a later analysis, especially for population genetics. This was a necessary step before intraspecific analyses could be done. Also, although unable to resolve all the relationships among the seamount ophiuroids, this study found a diverse assemblage of ophiuroids that may have radiated on the seamounts. 


\section{REFERENCES}

Avise, J. C. 1994. Molecular Markers, Natural History and Evolution. Chapman \& Hall.

BARIC, S., and C. Sturmbauer. 1999. Ecological Parallelism and Cryptic Species in the Genus Ophiothrix Derived from Mitochondrial DNA Sequences. Molecular Phylogenetics and Evolution 11: 157-162.

BARRETT, R., and P. HEBERT. 2005. Identifying spiders through DNA barcodes. Canadian Journal of Zoology 83: 481-491.

Chen, Z. Q., and K. J. MCNAmara. 2006. End-Permian extinction and subsequent recovery of the Ophiuroidea (Echinodermata). Palaeogeography, Palaeoclimatology, Palaeoecology 236: 321-344.

DeSalle, R., M. Egan, and M. SidDALl. 2005. The unholy trinity: taxonomy, species delimitation and DNA barcoding. Philosophical Transactions of the Royal Society B: Biological Sciences 360: 1905-1916.

DunCAN, R. A. 1984. Age progressive volcanism in the New England seamounts and the opening of the central Atlantic ocean. Journal of Geophysical Research 89: 99809990.

FELL, H. B. 1960. Synoptic Keys to the Genera of Ophiuroidea. Zoology Publications from Victoria University of Wellington 26: 1-44.

FRANCE, S. C., and L. L. HoOvER. 2002. DNA sequences of the mitochondrial COI gene have low levels of divergence among deep-sea octocorals (Cnidaria: Anthozoa). Hydrobiologia 471: 149-155.

Hebert, P., A. Cywinska, S. Ball, and J. DewaArd. 2003. Biological identifications through DNA barcodes. Proceedings of the Royal Society B: Biological Sciences 270: 313-321.

Hunter, R., and K. HalanyCH. 2008. Evaluating Connectivity in the Brooding Brittle Star Astrotoma agassizii across the Drake Passage in the Southern Ocean. Journal of Heredity 99: 137-148.

Katoh, K., K. MisAwA, K. Kuma, and T. MiyatA. 2002. MAFFT: a novel method for rapid multiple sequence alignment based on fast Fourier transform. Nucleic Acids Res 30: 3059-3066.

KIMURA, M. 1980. A simple method for estimating evolutionary rates of base substitutions through comparative studies of nucleotide sequences. Journal of Molecular Evolution 16: 111-120.

Le Gac, M., J. FÉral, E. Poulin, M. Veyret, and A. Chenuil. 2004. Identification of allopatric clades in the cosmopolitan ophiuroid species complex Amphipholis squamata (Echinodermata). The end of a paradox? Marine Ecology Progress Series 278: 171-178.

Lessios, H. A., B. D. Kessing, D. R. Robertson, and G. Paulay. 1999. Phylogeography of the pantropical sea urchin Eucidaris in relation to land barriers and ocean currents. Evolution 53: 806-817. 
Maddison, W. P., and D. R. MAdDison. 2000. MacClade. Sinauer Associates.

McCartney, M. A., G. Keller, and H. A. Lessios. 2000. Dispersal barriers in tropical oceans and speciation in Atlantic and eastern Pacific sea urchins of the genus Echinometra. Molecular Ecology 9: 1391-1400.

NeI, M., and S. KUMAR. 2000. Molecular evolution and phylogenetics. Oxford University Press.

Palumbi, S. R., A. Martin, S. Romano, W. O. McMillan, L. Stice, and G. GraBowsKI. 1991. The simple fool's guide to PCR, 2nd Ed. Dept. Zoology, University of Hawaii, .

PAterson, G. L. J. 1985. The deep-sea Ophiuroidea of the North Atlantic Ocean. Bulletin of the British Museum (Natural History) 49: 1-162.

RoY, M. S., and R. SPONER. 2002. Evidence of a human-mediated invasion of the tropical western Atlantic by "the world's most common brittle star". Proceedings of the Royal Society of London. B 269: 1017-1023.

Smith, A. B., G. J. L. PAterson, and B. LAfay. 1995. Ophiuroid phylogeny and higher taxonomy: morphological, molecular and palaeontological perspectives. Zoological Journal of the Linnean Society (prior to Jan 1, 2002) 114: 213.

SpONER, R., D. DEHEYN, and M. S. ROY. 2001. Large genetic distances within a population of Amphipholis squamata (Echinodermata; Ophiuroidea) do not support colour varieties as sibling species. Marine Ecology Progress Series 219: 169-175.

SPONER, R., and M. S. ROY. 2002. Phylogeographic analysis of the brooding brittle star Amphipholis squamata (echinodermata) along the coast of New Zealand reveals high cryptic genetic variation and cryptic dispersal potential. Evolution 56: 19541967.

Tamura, K., J. Dudley, M. Nei, and S. Kumar. 2007. MEGA4: Molecular Evolutionary Genetics Analysis (MEGA) software versin 4.0 Molecular Biology and Evolution 24: 1596-1599.

Tyler, P. A. 1980. Deep-Sea Ophiuroids. Oceanography and Marine Biology: An Annual Review 18: 125-153.

Walsh, P. S., D. A. Metzger, and R. Higuchi. 1991. Chelex 100 as a medium for simple extraction of DNA and PCR-based typing from forensic material. BioTechniques 10: 506-513.

WARD, R., T. ZEMLAK, B. InNes, P. LAst, and P. Hebert. 2005. DNA barcoding Australia's fish species. Philosophical Transactions of the Royal Society B: Biological Sciences 360: 1847-1857.

ZHENG, Y., and J. ARKANI-HAMED. 2002. Rigidity of the Atlantic oceanic lithosphere beneath New England seamounts. Tectonophysics 359: 359-369. 


\section{Chapter 4: Patterns of gene flow of four species of ophiuroids from North Atlantic seamounts}

\section{Introduction}

Seamounts are common geologic features on the seafloor with estimates of more than 800 seamounts in the Atlantic, 30,000-50,000 seamounts in the Pacific, and well more than 100,000 seamounts found in all the world oceans (Epp and Smoot 1989; Rogers 1994; Rogers 2004). They have been defined as mountains that rise more than $1000 \mathrm{~m}$ from the seafloor but do not rise above the sea surface and are often formed by mantle plumes associated with mid-plate hot spots, mid-ocean ridge volcanism, and more rarely by plate tectonic shifts (Batiza 2001; Epp and Smoot 1989; Fryer and Fryer 1987).

The mechanisms of faunal dispersal across ocean basins are key unknowns in our understanding of the present-day biogeography and biodiversity of deep-sea fauna. It has been proposed that seamounts play several important roles in faunal evolution, acting as “stepping-stones" for dispersal across ocean basins and boundary currents, regional centers of speciation, and refugia for populations with shrinking ranges (Hubbs 1959; Rogers 1994; Rogers 2004).

As hard substrate habitats dispersed throughout a typically sedimented deep ocean, seamounts may be key links between different biogeographic provinces within an ocean. Seamounts are often formed in chains perpendicular to coastlines and mid-ocean ridges. These may be important faunal passages for dispersal as was found in a recent survey of fauna from Bear seamount of the New England Seamount chain in the Atlantic Ocean that found several species of fish previously thought to occur only in the eastem Atlantic, indicating that this seamount chain may serve as a westward route of dispersal for eastern Atlantic fauna (Moore et al. 2003).

Seamounts may be significant links between different biogeographic provinces within the oceans acting as "stepping-stones" and playing an important part in the transoceanic dispersal of organisms as key faunal passages for dispersal (Hamilton 1956; 
Hubbs 1959; Rogers 1994). The concentration of commercially valuable fish at seamounts such as the orange roughy, hoki, oreosomatids, Patagonian toothfish, and pelagic armourheads has been well documented (Koslow et al. 2000; Rogers 1994). With the collapse of traditional continental shelf and pelagic fisheries and the improvement of fishing technology, deep-sea fisheries have grown and have contributed 800,000 to 100,000 tons annually to global fish landings since 1964 (Clark 2001; Koslow et al. 2000). Deep-sea fishing techniques such as trawling and long-line fishing have been shown to destroy benthic habitat, in particular the coral habitat upon which many invertebrates live (Kaiser 1998; Probert 1999; Probert et al. 1997; Thrush et al. 1998). With expanding deep-sea fisheries and the concurrent destruction of seamount habitat, particularly of the live and fossil corals, conservation efforts for these areas have increased like the proposal for the precautionary closure of four seamount areas in the North Atlantic to deep-sea fisheries, which include portions of the Corner and New England seamounts (Nafo 2006). Understanding the extent of genetic connectivity between seamounts and seamount areas will aid in the creation of marine protected areas and future conservation and policy efforts. Such a strategy is advocated for the conservation of coral reef communities (Palumbi 2003; Richards et al. 2007).

Seamounts have been shown to support large faunal abundances, particularly of deep-sea coral communities and commercially valuable fish (Rogers 1994; Wilson and Kaufmann 1987). Related to this is the evidence for a high degree of endemism found at seamounts, indicating that they may serve as areas of speciation or refugia for relict populations (Rogers 1994; Rogers 2004). Wilson and Kaufman (1987) estimated that $12 \%$ of all fish found on seamounts were endemic while the invertebrate fauna was estimated to have a $15 \%$ level of endemism. The level of fish endemism on the Hawaiian and Emperor seamount chains have been shown to be 17\% (Stocks 2003) while a study at the Great Meteor seamount found fish endemism to be 9\% (Fock et al. 2002). Koslow et al. (2001) found higher levels on the seamounts off Tasmania, which had endemism levels of 35\%. De Forges et al. (2000) found that of the macro- and megafauna of seamounts in the Tasman Sea and southeast Coral Sea, 29-34\% were potential seamount 
endemics. Even higher rates were found on the Nasca and Sala-y-Gomez seamount chains off the coast of Chile, which had endemism levels for fish of $44 \%$ and $52 \%$ for invertebrates (Parin et al. 1997). These high levels of endemism are not unique to large and isolated seamounts as would be expected. The Tasmanian seamounts have an average height of $400 \mathrm{~m}$ (Koslow 2001) and seamounts with heights of only 100-350 m near the Chatham Rise have rates of endemism of $15 \%$ (Rowden et al. 2002). This would suggest that seamounts of many sizes serve as potential area of speciation where such high levels of endemism can occur. These high levels of endemism are also an indication of the isolation of seamounts. This is shown through the limited number of species shared by even adjacent seamounts, as was found for adjacent New Caledonian seamounts, which shared only $21 \%$ of species sampled from them (Richer De Forges et al. 2000).

To infer processes underlying patterns responsible for structuring endemism, morphological and genetic studies have looked at the connectivity between populations on different seamounts with varying results. The majority of studies targeting fish and larger mobile fauna have shown no genetic or geographical isolation between seamounts. Studies of the lightfish, Maurolicus muelleri (Gmelin), found that the population at the Southeast Hancock seamount in the Pacific Ocean was made up primarily of juveniles and that there were not enough adults to support a self-sustaining population, indicating possible recruitment from the southern Emperor seamount populations (Boehlert et al. 1994). Another study also found that there was no increase of concentrations of larval fish of seamount-associated species over seamounts versus away from a seamount (Boehlert 1988). Tagging studies of individual yellowfin and bigeye tuna also found that they moved from seamount areas to non-seamount areas (Sibert et al. 2000). Genetic studies on fish have also found that there are no differences between different populations found on neighboring seamounts and adjacent non-seamount areas (Bolsch et al. 1993; Borets 1979).

Several studies have also looked at the morphological differences between populations found on different seamount and non-seamount areas. Boehlert et al. (1994) 
found that the Southeast Hancock Seamount population of the adult lightfish, Maurolicus muelleri (Gmelin), differed in the number of gillrakers from Japanese populations. Wilson and Kaufman (1987) found similar morphological differences in Northeast Atlantic seamount populations of the frostfish, Lepidopus caudatus with distinct northern seamount and southern seamount morphotypes. Similar results were found between the different colonies of the coral Ellisella flagellum on the Great Meteor Seamount and on the Josephine Seamount (Grasshoff 1972).

Genetic studies have also shown differentiation between seamount and nonseamount populations. A study of the deep-sea amphipod Eurythenes gryllus found that individuals from the summit of Horizon Guyot were genetically distinct from individuals near the base of the guyot and at other deep-sea sites (Bucklin et al. 1987). It was further found that there was genetic homogeneity of E. gryllus populations within the same depth zone at the scale of ocean basins, but genetically distinct populations at different depths, indicating that depth may be an important factor influencing population structure of seamount fauna (France and Kocher 1996). A study of the coral Corallium lauuense using microsatellites found low but significant levels of population differentiation among several seamounts and islands in the Hawaiian Archipelago (Baco and Shank 2005). A study of invertebrates from the Norfolk seamounts in the Southwest Pacific found no genetic structure for the two galatheid crabs Munida thoe and Munida zebra, the two chirostylid crab Eumunida species and one plaktotrophic gastropod Sassia remensa, but significant structure for the non-planktotrophic gastropod Nassaria probelmatica (Samadi et al. 2006). These studies suggest that there is some degree of isolation of seamounts depending on the taxa studied.

The North Atlantic seamounts include three seamount groups, the New England seamount chain the Corner Rise seamounts, and Muir seamount (Figure 1). These seamounts provide a natural laboratory of a seamount chain and group seemingly hydrographically separated. The New England seamount chain is the longest seamount chain in the North Atlantic consisting of about 35 major peaks with elevations ranging from 400-4000 m. The seamount chain extends $1200 \mathrm{~km}$ along a northwest-southeast 
direction, beginning with Bear seamount, located on the continental slope south of the Georges Bank, and ending with Nashville seamount at the end of the Bermuda Rise (Zheng and Arkani-Hamed 2002). The Corner Rise seamounts are a cluster of seamounts located between the Mid-Atlantic Ridge and the New England seamount chain. The Corner Rise seamounts are located approximately $300 \mathrm{~km}$ east of the New England seamounts and consists of about 50 seamounts (Cairns 2007). Additionally, Muir seamount is a distant seamount located north of Bermuda.

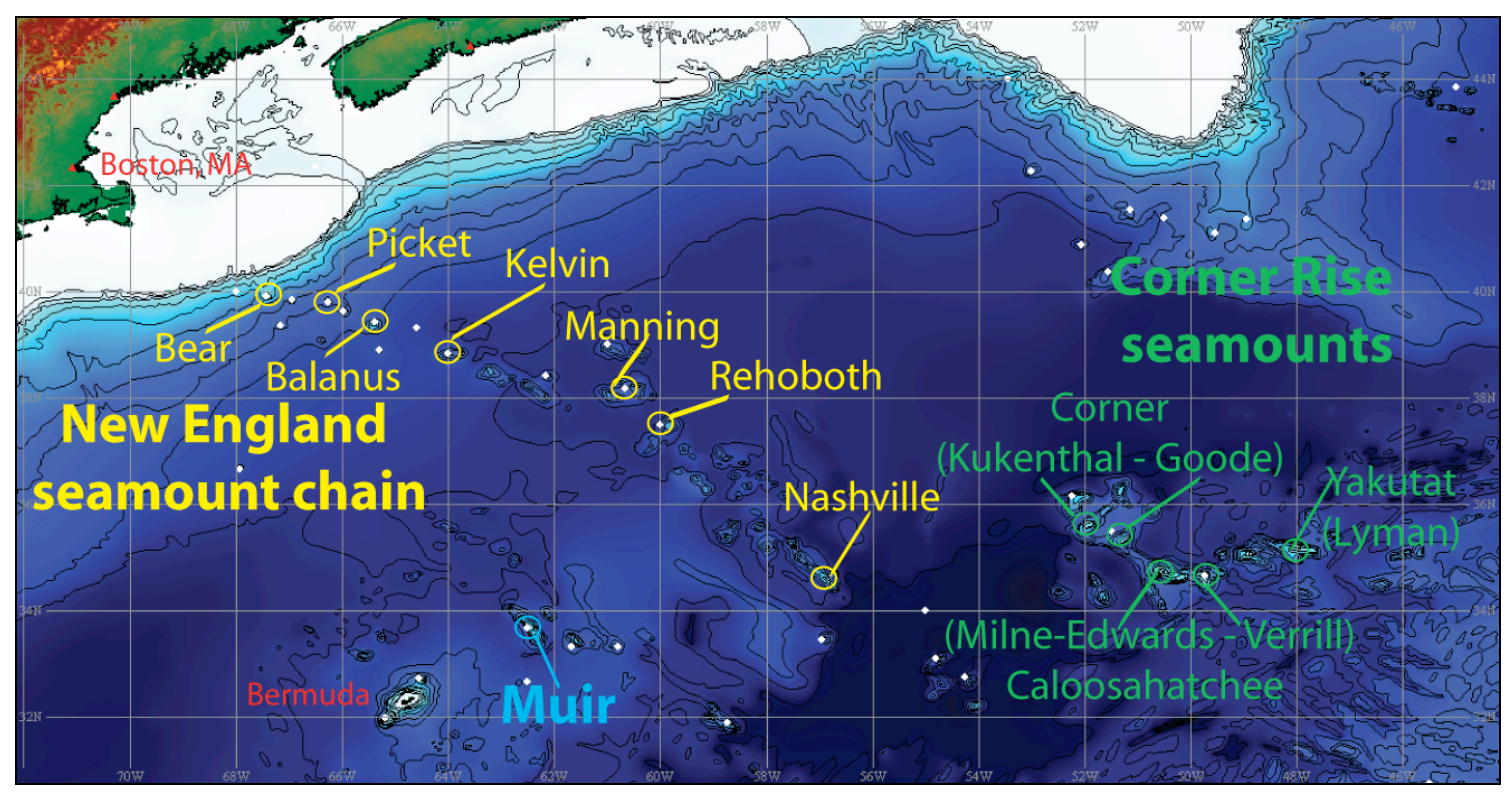

Figure 1. Map of sampled seamounts in the North Atlantic. The New England seamount chain is in yellow, the Corner Rise seamounts in green, and Muir seamount is in blue.

The New England seamounts have been shown to interact with the currents of the region as well, in particular influencing the Gulf Stream, which bisects the seamount chain. This interaction also results in gyres with circulations occurring in opposite directions which could potentially effect the dispersal of seamount fauna (Figure 2)(Ezer 1994; Qiu 1994). 


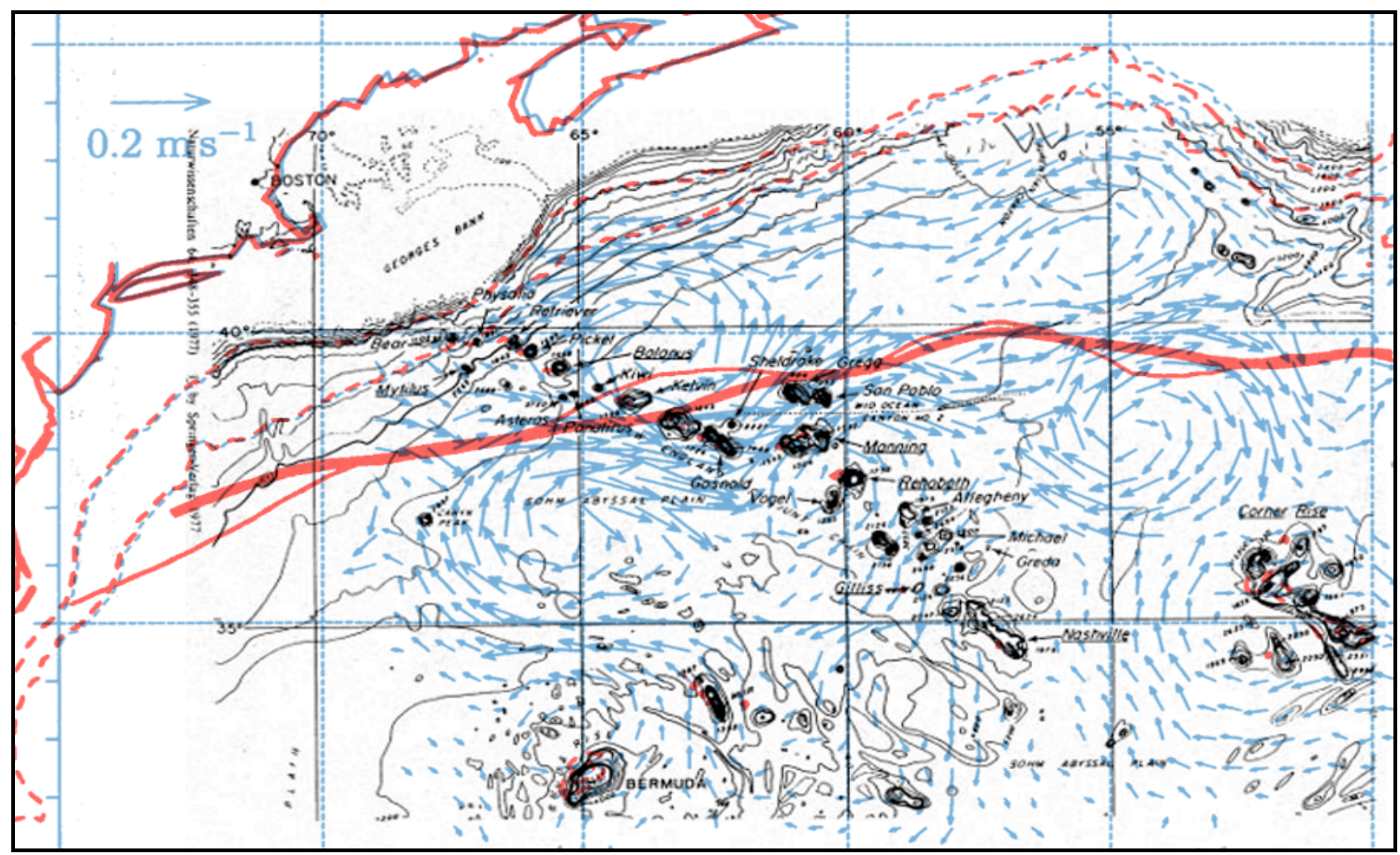

Figure 2. North Atlantic seamounts with the mean Gulf Stream in red and flow patterns at $3000 \mathrm{~m}$ depth in blue; calculated from modeling studies using hydrographic and altimetric data. Image modified from (Houghton et al. 1977; Qiu 1994).

The interacting biological, physical, and hydrological characteristics associated with Seamounts in the North Atlantic suggests that the New England and Corner Rise seamounts are key locations to examine their ability to impart important factors controlling marine biodiversity, biogeography, and evolution.

In order to assess the degree to which North Atlantic seamounts act as steppingstones of dispersal and the level of population connectivity and gene flow among the North Atlantic a multi-gene, multi-species approach was utilized to examine four species of ophiuroids, specifically with regard to varying levels of associations with coral hosts using two mitochondrial molecular markers, $16 \mathrm{~S}$ and COI. More than 20 potential species of ophiuroids were found on the North Atlantic seamounts. Both $16 \mathrm{~S}$ and COI have been used in genetic studies of ophiuroids in the past, although COI has been a particularly difficult gene to work with within ophiuroids (Sponer et al. 2001). These markers have been used to identify cryptic species in ophiuroids in several cases (Baric and Sturmbauer 1999; Hunter and Halanych 2008; Roy and Sponer 2002; Sponer et al. 2001; Sponer and 
Roy 2002). The different host-associate relationships provided the opportunity to target these ecological differences using population genetic approaches.

Recent ecological theory has focused on the importance of facilitation in controlling structure and function of aquatic communities (Bruno et al. 2003; Richards et al. 2007). In order to look at this more closely we examined four of the 22 ophiuroid species found on the North Atlantic seamounts with varying degrees of association with coral hosts in order to determine the gene flow among seamount populations and the effect facilitation may have on that genetic structure. Asteroschema clavigera Verrill, 1894 is an ophiuroid strongly associated with the gorgonian corals Paramuricea sp. or Paragorgia sp., being collected only from these coral species and never observed apart from them usually with one to two individuals per Paramuricea sp. and with many individuals on Paragorgia sp. (Chapter 2). A similar association has been shown for another Asteroschema species, Asteroschema tenue, with the gorgonian coral Ellisella barbadensis (Emson and Woodley 1987). Ophiocreas oedipus Lyman, 1879 is an ophiuroid associated whereby $99.8 \%$ of the time, only a single individual is intertwined with the host coral Metallogorgia melanotrichos (only one time were two observed on one host) (Chapter 2). Ophioplinthaca abyssalis Cherbonnier \& Sibouet, 1972 is an ophiuroid that predominantly inhabits the coral host Candidella imbricata in tens of individuals, but this species has also been observed on other hosts (Chapter 2).

Ophioplinthaca chelys Wyville Thomson, 1878 is an ophiuroid that lives in the benthic habitats without an association with any particular host (Chapter 2). (A. B. Smith 1995).

In order to maximize the genetic signal from ophiuroid species, I analyzed four species with different "habitat strategies" which limited the samples available for this study. In addition, the sample sizes were limited by the difficulty of sample collection in the deep-sea. However, previous studies have shown that only a small number of sequences may be needed for obtaining reliable species geneaologies in sequence-based population genetic studies (Hey and Kliman 1993; Kliman and Hey 1993).

Congruent patterns of genetic differentiation and gene flow among the four species will help guide conservation efforts in this region. It is expected that $A$. clavigera and $O$. 
oedipus will have similar patterns of dispersal as both have relatively tight associations with coral hosts and may be influenced by the same factor of having to find their appropriate coral host. The ophioplinthacid species may differ, especially $O$. chelys as it shows no association with any host. The results of this study will provide insight into fauna associated with coral communities and those that may not be as well.

\section{Materials and Methods}

\section{Sample collection}

Individuals of four ophiuroid species were collected from 13 seamount peaks designated as populations in the North Atlantic (Table 1). Most populations were sampled during the 2005 Deep Atlantic Stepping Stones research expedition aboard the $R / V$ Ron Brown using the ROV Hercules. The ROV Hercules was equipped with a Kraft Predator force-reflecting manipulator arm, an ISE Magnum manipulator arm, various adaptors for the manipulator arms for use during collection, insulated bioboxes for sample collection, and two suction pumps. The manipulator arm was modified with cutting blades to aid in the collection of corals and other invertebrates. 
Table 1. Geographic location, number of individuals, and gene region examined from seamount populations used in this study.

\begin{tabular}{|c|c|c|c|c|c|c|c|}
\hline \multirow[b]{2}{*}{$\begin{array}{l}\text { Geographic } \\
\text { Region }\end{array}$} & \multirow[b]{2}{*}{$\begin{array}{l}\text { Seamount } \\
\text { peak }\end{array}$} & \multicolumn{4}{|c|}{$16 S$} & \multicolumn{2}{|c|}{$\mathrm{COI}$} \\
\hline & & A. clavigera & $\begin{array}{l}\text { O. } \\
\text { oedipus }\end{array}$ & $\begin{array}{l}\text { O. } \\
\text { abyssalis }\end{array}$ & O. chelys & A. clavigera & $\begin{array}{l}\text { O. } \\
\text { oedipus }\end{array}$ \\
\hline \multirow{8}{*}{$\begin{array}{l}\text { New England } \\
\text { Seamount Chain }\end{array}$} & & & & & & & \\
\hline & Bear & 6 & 1 & - & - & - & - \\
\hline & Picket & 5 & - & - & 1 & - & - \\
\hline & Balanus & 1 & 1 & 15 & 1 & 1 & 2 \\
\hline & Kelvin & 7 & 2 & 21 & 9 & 4 & 1 \\
\hline & Rehoboth & 10 & 5 & 20 & 11 & 4 & 4 \\
\hline & Manning & 35 & 1 & - & 1 & 4 & - \\
\hline & Nashville & 3 & 5 & 10 & - & 3 & 3 \\
\hline \multirow[t]{5}{*}{ Corner Rise } & Kukenthal & 1 & 1 & 4 & - & 1 & 1 \\
\hline & Goode & 4 & 4 & 19 & 8 & 1 & 4 \\
\hline & $\begin{array}{l}\text { Verrill } \\
\text { Milne- }\end{array}$ & 2 & 2 & - & 8 & 2 & 2 \\
\hline & Edwards & - & 2 & - & - & - & 1 \\
\hline & Lyman & 2 & 1 & - & 7 & 1 & 1 \\
\hline Muir & Muir & 8 & 3 & - & - & - & 1 \\
\hline
\end{tabular}

The four target ophiuroids species were: Asteroschema clavigera Verrill, 1894, Ophiocreas oedipus Lyman, 1879, Ophioplinthaca abyssalis Cherbonnier \& Sibouet, 1972, and Ophioplinthaca chelys Wyville Thomson, 1878 were collected in 2003 and 2005 using the DSV Alvin and the ROV Hercules, respectively. Individuals were processed immediately following the recovery of the ROV Hercules. The ophiuroids were initially sorted on board the ship as preliminary morphospecies. Arms of individuals identified as $A$. clavigera and $O$. oedipus were subsampled and frozen for later genetic analysis while the remaining whole individuals were preserved in $(4 \%$ formalin, and transferred to $70 \%$ ethanol after 24 hours). Whole individuals of $O$. abyssalis and $O$. chelys were frozen for subsequent genetic analysis. Museum voucher specimens were provided to the Peabody museum and the Smithsonian Institution for morphological determination of species identification. Ophiuroids collected in 2003 during research cruise AT11-7 aboard the $R / V$ Atlantis using the DSV Alvin. These samples provided additional ophiuroid population from Manning Seamount as well as populations from Muir seamount. 
Additional ophiuroid specimens were preserved in $70 \%$ ethanol from Bear Seamount obtained on research cruise DE 02-06 in 2002 aboard the $R / V$ Delaware II. Samples from Picket seamount were processed and frozen at $-70^{\circ} \mathrm{C}$ following collection by $D S V$ Alvin in 2005 during research cruise AT12-1.

\section{DNA extraction, polymerase chain reaction, and sequencing}

Genomic ophiuroid DNA was extracted using a 5\% Chelex extraction protocol (Sponer and Roy 2002; Walsh et al. 1991). A small piece of tissue ranging from 1-3 mm of an arm was minced and then added to $500 \mu 1$ of $5 \%$ Chelex (Bio-Rad Hercules, CA) solution. This was heated for $3 \mathrm{~h}$ at $65^{\circ} \mathrm{C}$ with the issue completely submerged within the chelex beads. The solution was then vortexed and heated to $95^{\circ}-100^{\circ} \mathrm{C}$ for 10 minutes and then centrifuged at 13,500×g for 10 minutes. Ethanol-preserved samples were processed in the same way with the addition of an initial rinse of the tissue in water. The extractions were stored in $4^{\circ} \mathrm{C}$ for short-term storage and $-20^{\circ} \mathrm{C}$ for long-term storage.

Several molecular markers were pursued for use as informative population genetic markers, including 16S, COI, COX2, NAD5, ATP6, ITS, and microsatellites. Most of these markers were either too inconsistent in amplification or invariable to use in this study. Three mitochondrial DNA molecular markers were chosen from this set that consistently amplified gene fragments from the four species of ophiuroids. One fragment from the 16S rRNA mitochondrial gene was amplified for all four species and one fragment from the COI mitochondrial gene was amplified for A. clavigera and O. oedipus (Table 2).

Table 2. Gene fragment length (and their respective primers) analyzed for four species of ophiuroids.

\begin{tabular}{|c|c|c|}
\hline Species & $\begin{array}{c}\text { 16S fragment length } \\
\text { (bp) (16Sar/16Sbr) }\end{array}$ & $\begin{array}{c}\text { COI length (bp) } \\
\text { (mCOIF100/COIr1340a) }\end{array}$ \\
\hline A. clavigera & 429 & 1083 \\
\hline O. oedipus & 428 & 1117 \\
\hline O. abyssalis & 474 & - \\
\hline O. chelys & 476 & - \\
\hline
\end{tabular}


A fragment of the mitochondrial DNA gene 16S rRNA (16S) was amplified with the primers 16SarL (5'-CGCCTGTTTATCAAAAACAT-3') and 16SbrH (5'CCGGTCTGAACTCAGATCACGT-3’) (Palumbi et al. 1991). These primers worked consistently for all four species and were used for sequencing gene fragments of varying sizes depending on the species (Table 2). Most fragments were amplified in $25 \mu 1 \mathrm{PCR}$ reactions containing 1X PCR buffer (Promega), $2.5 \mathrm{mM} \mathrm{MgCL} 2,1.0 \mathrm{mM}$ of each dNTP, $0.5 \mu \mathrm{M}$ of each primer, $0.5 \mathrm{M}$ Betaine, $1-2 \mu \mathrm{l}$ of extracted template, and $0.2 \mathrm{U}$ of Taq polymerase (Promega). Some fragments were amplified using 1X PCR Buffer (Eppendorf), $2.5 \mathrm{mM} \mathrm{MgCL} 2,1.0 \mathrm{mM}$ of each dNTP, $0.5 \mu \mathrm{M}$ of each primer, $1 \mathrm{X}$ TaqMaster PCR Enhancer (Eppendorf), 1-2 $\mu \mathrm{l}$ of extracted template, and 0.2U of Taq polymerase (Eppendorf). PCR was performed under the following conditions: initial denaturation for $2 \mathrm{~min}$ at $94^{\circ} \mathrm{C}$, followed by 30 cycles of denaturation at $94^{\circ} \mathrm{C}$ for $1 \mathrm{~min}$, 30 cycles of annealing at $45^{\circ} \mathrm{C}$ for $1 \mathrm{~min}$, and 30 cycles of extension at $72^{\circ} \mathrm{C}$ for $45 \mathrm{sec}$, followed by a final extension phase of $72^{\circ} \mathrm{C}$ for $7 \mathrm{~min}$.

A fragment of the mitochondrial COI gene was amplified for analyses from A.clavigera and O. oedipus samples using the primers mCOIF100 for (5'GCCGGAACTGTAGGAACAG-3') and COIR1340a rev (5'GAGTATCGTCGGGGCAT-3') developed by Dr. Paula Cisternas (personal communication). These primers amplified approximately $1000 \mathrm{bp}$ of the COI gene for both species (Table 2). These fragments were amplified under the same conditions as described above except for modifications to the annealing temperature. Samples of $A$. clavigera were amplified with an annealing temperature of $56^{\circ} \mathrm{C}$ and $O$. oedipus were amplified with an annealing temperature of $50^{\circ} \mathrm{C}$. PCR reactions were similar to those above.

Successfully amplified PCR products of 16S were directly purified using either the QIAquick PCR Purification kit (Qiagen) or the SV Gel and PCR Cleanup Kit (Promega) and eluted into a final $30 \mu \mathrm{l}$ volume. PCR products of COI were gel purified using the QIAquick Gel Extraction kit (Qiagen) and the SV Gel and PCR Cleanup Kit (Promega) and eluted into a final $30 \mu \mathrm{l}$ volume. Purified PCR products were then used in 
one-eighth format sequencing reactions using Big Dye terminators (version 3, PerkinElmer) in 96-well plates and purified by isopropanol precipitation before sequencing on an ABI 3700 Capillary Sequencer. PCR products were sequenced bi-directionally except for 13 samples where only one direction could be successfully obtained.

\section{Population Structure}

Sequences were edited in Sequencher 4.8 (Gene Codes Corporation Inc.) and aligned using MAFFT v6.527 (Katoh et al. 2002). Alignments were viewed by eye in MacClade 4.08 (Maddison and Maddison 2000), and COI sequences were translated to ensure that stop codons were not present. The following analyses were performed for each species and each molecular marker separately (the four 16S datasets for $A$.

clavigera, O. oedipus, O. abyssalis, and O. chelys; the two COI fragment 1 datasets for $A$. clavigera and $O$. oedipus).

Parsimony networks were constructed with mtDNA haplotypes within each species and for each molecular marker. This was done in TCS 1.21 (Clement et al. 2000), using a 95\% connection limit between haplotypes and treating gaps as a fifth state. The network was then transformed into a geographic map of haplotype distributions.

Analyses of molecular data were conducted within each species and for each molecular marker separately using Arlequin v3.1 (Excoffier et al. 2005). Intra-specific genetic diversity was evaluated by computing the number of haplotypes, the number of polymorphic sites, haplotype diversity $(h)$, and nucleotide diversity $(\pi)$. Pairwise $\Phi_{\mathrm{ST}}$ were computed between the seamount peak populations creating a genetic distance matrix in Arlequin v3.1 (Excoffier et al. 2005). The pairwise $\Phi_{\mathrm{ST}}$ values were used in an analysis of molecular variance (AMOVA) implemented with Arlequin to evaluate the geographic isolation of populations and regional groups. The AMOVA was performed with three hierarchical levels: within each seamount peak, among seamount peaks but within regional groups, and among regional groups where the regional groups were defined as the New England Seamount chain, the Corner Rise seamounts, and Muir 
seamount. The pairwise $\Phi_{\mathrm{ST}}$ and AMOVA were each performed with 10,000 permutations and the Kimura-2-parameter model (Kimura 1980).

We used the Mantel test (Mantel 1967) within Arlequin to test isolation-bydistance, comparing the matrix of Kimura-2-parameter genetic distances between seamount peak populations and a matrix of direct (over water) geographical distances between seamount peaks.

Tajima's D (Tajima 1989) test statistic was calculated within Arlequin to test the assumption of selective neutrality of mtDNA sequences. Mismatch analyses were also performed in Arlequin investigating the mismatch distributions of pairwise differences between all individuals of each population for the seven different mtDNA datasets, in order to assess the extent to which populations have undergone a rapid expansion or remained stable over time (Aboim et al. 2005).

Levels of migration between pairs of populations were estimated in the software package MIGRATE version 3.0 using the Bayesian framework (Beerli and Felsenstein 2001). Uniform prior distributions were used for the parameters $\theta$ (minimum $=0.005$, maximum $=0.10)$ and $\mathrm{M}($ minimum $=0$, maximum $=1000)$. I used one long chain, 1000000 steps sampled, with a burn-in of 10000 and an adaptive heating scheme with start temperatures of 1.0, 1.5, 3.0,10000).

\section{Results}

From the 13 seamount populations, we successfully amplified a total of 83 individuals of $A$. clavigera, 28 individuals of $O$. oedipus, 89 individuals of $O$. abyssalis, and 46 individuals of $O$. chelys for $16 \mathrm{~S}$. We obtained 21 sequences of COI from $A$. clavigera, and 20 sequences from O. oedipus (Table 1). The haplotypic diversity of COI was generally higher than that for $16 \mathrm{~S}$ in A. clavigera and O. oedipus. Also, the $16 \mathrm{~S}$ haplotype diversity of $O$. abyssalis was generally higher among seamount populations than that for A. clavigera (Table 3). 
Table 3. Sample size and summary statistics of $16 \mathrm{~S}$ and $\mathrm{COI}$ fragments for each analyzed species from the New England and Corner Rise seamounts. NESC $=$ New England seamount chain

\begin{tabular}{|c|c|c|c|c|c|c|c|c|}
\hline Species & Gene & $\begin{array}{l}\text { Geographic } \\
\text { Region }\end{array}$ & $\begin{array}{l}\text { Seamount } \\
\text { peak }\end{array}$ & $\begin{array}{c}\text { Sample } \\
\text { Size }\end{array}$ & $\begin{array}{l}\text { Polymorphic } \\
\text { sites } \\
\end{array}$ & $\begin{array}{c}\text { Haplotype } \\
\text { number }\end{array}$ & Haplotype diversity (h) & Nucleotide diversity $(\pi)$ \\
\hline \multirow[t]{13}{*}{ A. clavigera } & $16 S$ & NESC & Bear & 6 & 2 & 3 & $0.6000+/-0.2152$ & $0.001569+/-0.001594$ \\
\hline & & & Picket & 5 & 3 & 3 & $0.7000+/-0.2184$ & $0.002833+/-0.002505$ \\
\hline & & & Balanus & 1 & 0 & 1 & $1.0000+/-0.0000$ & 0 \\
\hline & & & Kelvin & 7 & 3 & 3 & $0.5238+/-0.2086$ & $0.001343+/-0.001400$ \\
\hline & & & Rehoboth & 10 & 8 & 5 & $0.6667+/-0.1633$ & $0.002835+/-0.002225$ \\
\hline & & & Manning & 35 & 9 & 11 & $0.6353+/-0.0911$ & $0.001923+/-0.001569$ \\
\hline & & & Nashville & 3 & 1 & 2 & $0.6667+/-0.3143$ & $0.001568+/-0.001954$ \\
\hline & & Corner Rise & Kukenthal & 1 & 0 & 1 & $1.0000+/-0.0000$ & $0.000000+/-0.000000$ \\
\hline & & & Goode & 3 & 1 & 3 & $1.0000+/-0.2722$ & $0.001594+/-0.001987$ \\
\hline & & & $\begin{array}{l}\text { Verrill } \\
\text { Milne- }\end{array}$ & 2 & 2 & 2 & $1.0000+/-0.5000$ & $0.002347+/-0.003318$ \\
\hline & & & Edwards & - & - & - & - & - \\
\hline & & & Lyman & 2 & 1 & 2 & $1.0000+/-0.5000$ & $0.002353+/-0.003326$ \\
\hline & & Muir & Muir & 8 & 3 & 4 & $0.7500+/-0.1391$ & $0.002186+/-0.001903$ \\
\hline \multirow[t]{13}{*}{ A. clavigera } & $\mathrm{COI}$ & NESC & Bear & - & - & - & - & - \\
\hline & & & Picket & - & - & - & - & - \\
\hline & & & Balanus & 1 & 0 & 1 & $1.0000+/-0.0000$ & $0.000000+/-0.000000$ \\
\hline & & & Kelvin & 4 & 12 & 4 & $1.0000+/-0.1768$ & $0.005788+/-0.004156$ \\
\hline & & & Rehoboth & 4 & 7 & 4 & $1.0000+/-0.1768$ & $0.003247+/-0.002482$ \\
\hline & & & Manning & 4 & 4 & 4 & $1.0000+/-0.1768$ & $0.001855+/-0.001555$ \\
\hline & & & Nashville & 3 & 7 & 3 & $1.0000+/-0.2722$ & $0.004650+/-0.003858$ \\
\hline & & Corner Rise & Kukenthal & 1 & 0 & 1 & $1.0000+/-0.0000$ & $0.000000+/-0.000000$ \\
\hline & & & Goode & 1 & 0 & 1 & $1.0000+/-0.0000$ & $0.000000+/-0.000000$ \\
\hline & & & $\begin{array}{l}\text { Verrill } \\
\text { Milne-- }\end{array}$ & 2 & 10 & 2 & $1.0000+/-0.5000$ & $0.009321+/-0.009772$ \\
\hline & & & Edwards & - & - & - & - & - \\
\hline & & & Lyman & 1 & 0 & 1 & $1.0000+/-0.0000$ & $0.000000+/-0.000000$ \\
\hline & & Muir & Muir & - & - & - & - & - \\
\hline \multirow[t]{3}{*}{ O. oedipus } & $16 S$ & NESC & Bear & 1 & 0 & 1 & $1.0000+/-0.0000$ & $0.000000+/-0.000000$ \\
\hline & & & Picket & & & & - & - \\
\hline & & & Balanus & 1 & 0 & 1 & $1.0000+/-0.0000$ & $0.000000+/-0.000000$ \\
\hline
\end{tabular}


Table 3. continued

\begin{tabular}{|c|c|c|c|c|c|c|c|c|}
\hline Species & Gene & $\begin{array}{l}\text { Geographic } \\
\text { Region }\end{array}$ & $\begin{array}{l}\text { Seamount } \\
\text { peak }\end{array}$ & $\begin{array}{c}\text { Sample } \\
\text { Size }\end{array}$ & $\begin{array}{c}\text { Polymorphic } \\
\text { sites }\end{array}$ & $\begin{array}{c}\text { Haplotype } \\
\text { number }\end{array}$ & Haplotype diversity (h) & Nucleotide diversity $(\pi)$ \\
\hline & & & Kelvin & 2 & 0 & 1 & $0.0000+/-0.0000$ & $0.000000+/-0.000000$ \\
\hline & & & Rehoboth & 5 & 2 & 3 & $0.7000+/-0.2184$ & $0.001875+/-0.001865$ \\
\hline & & & Manning & 1 & 0 & 1 & $1.0000+/-0.0000$ & $0.000000+/-0.000000$ \\
\hline & & & Nashville & 5 & 0 & 1 & $0.0000+/-0.0000$ & $0.000000+/-0.000000$ \\
\hline & & Corner Rise & Kukenthal & 1 & 0 & 1 & $1.0000+/-0.0000$ & $0.000000+/-0.000000$ \\
\hline & & & Goode & 4 & 1 & 2 & $0.5000+/-0.2652$ & $0.001173+/-0.001453$ \\
\hline & & & $\begin{array}{l}\text { Verrill } \\
\text { Milne- }\end{array}$ & 2 & 0 & 1 & $0.0000+/-0.0000$ & $0.000000+/-0.000000$ \\
\hline & & & Edwards & 2 & 0 & 1 & $0.0000+/-0.0000$ & $0.000000+/-0.000000$ \\
\hline & & & Lyman & 1 & 0 & 1 & $1.0000+/-0.0000$ & $0.000000+/-0.000000$ \\
\hline & & Muir & Muir & 1 & 0 & 1 & $0.0000+/-0.0000$ & $0.000000+/-0.000000$ \\
\hline \multirow[t]{13}{*}{ O. oedipus } & $\mathrm{COI}$ & NESC & Bear & - & - & - & - & - \\
\hline & & & Picket & - & - & - & - & - \\
\hline & & & Balanus & 2 & 0 & 1 & $0.0000+/-0.0000$ & $0.000000+/-0.000000$ \\
\hline & & & Kelvin & 1 & 0 & 1 & $1.0000+/-0.0000$ & $0.000000+/-0.000000$ \\
\hline & & & Rehoboth & 4 & 4 & 4 & $1.0000+/-0.1768$ & $0.001801+/-0.001510$ \\
\hline & & & Manning & - & - & - & - & - \\
\hline & & & Nashville & 3 & 4 & 3 & $1.0000+/-0.2722$ & $0.002392+/-0.002146$ \\
\hline & & Corner Rise & Kukenthal & 1 & 0 & 1 & $1.0000+/-0.0000$ & $0.000000+/-0.000000$ \\
\hline & & & Goode & 4 & 2 & 2 & $0.5000+/-0.2652$ & $0.000897+/-0.000888$ \\
\hline & & & $\begin{array}{l}\text { Verrill } \\
\text { Milne- }\end{array}$ & 2 & 5 & 2 & $1.0000+/-0.5000$ & $0.004493+/-0.004920$ \\
\hline & & & Edwards & 1 & 0 & 1 & $1.0000+/-0.0000$ & $0.000000+/-0.000000$ \\
\hline & & & Lyman & 1 & 0 & 1 & $1.0000+/-0.0000$ & $0.000000+/-0.000000$ \\
\hline & & Muir & Muir & 1 & 0 & 1 & $1.0000+/-0.0000$ & $0.000000+/-0.000000$ \\
\hline \multirow[t]{9}{*}{ O, abyssalis } & $16 S$ & NESC & Bear & - & - & - & - & - \\
\hline & & & Picket & - & - & - & - & - \\
\hline & & & Balanus & 15 & 8 & 6 & $0.8667+/-0.0483$ & $0.005324+/-0.003417$ \\
\hline & & & Kelvin & 21 & 12 & 10 & $0.8190+/-0.0683$ & $0.003737+/-0.002529$ \\
\hline & & & Rehoboth & 20 & 4 & 5 & $0.7789+/-0.0524$ & $0.002677+/-0.001977$ \\
\hline & & & Manning & - & - & - & - & - \\
\hline & & & Nashville & 10 & 7 & 6 & $0.8444+/-0.1029$ & $0.004558+/-0.003133$ \\
\hline & & Corner Rise & Kukenthal & 4 & 3 & 2 & $0.6667+/-0.2041$ & $0.004357+/-0.003651$ \\
\hline & & & Goode & 19 & 9 & 8 & $0.8655+/-0.0458$ & $0.004928+/-0.003161$ \\
\hline
\end{tabular}


Table 3. continued

\begin{tabular}{|c|c|c|c|c|c|c|c|c|}
\hline Species & Gene & $\begin{array}{l}\text { Geographic } \\
\text { Region }\end{array}$ & $\begin{array}{l}\text { Seamount } \\
\text { peak }\end{array}$ & $\begin{array}{c}\text { Sample } \\
\text { Size }\end{array}$ & $\begin{array}{c}\text { Polymorphic } \\
\text { sites } \\
\end{array}$ & $\begin{array}{c}\text { Haplotype } \\
\text { number }\end{array}$ & Haplotype diversity (h) & Nucleotide diversity $(\pi)$ \\
\hline & & & $\begin{array}{l}\text { Verrill } \\
\text { Milne- } \\
\text { Edwards }\end{array}$ & - & - & - & - & - \\
\hline & & & Lyman & - & - & - & - & - \\
\hline & & Muir & Muir & - & - & - & - & - \\
\hline \multirow[t]{12}{*}{ O. chelys } & $16 S$ & NESC & Bear & - & - & - & - & - \\
\hline & & & Picket & 1 & 0 & 1 & $1.0000+/-0.0000$ & $0.000000+/-0.000000$ \\
\hline & & & Balanus & 1 & 0 & 1 & $1.0000+/-0.0000$ & $0.000000+/-0.000000$ \\
\hline & & & Kelvin & 9 & 2 & 4 & $0.5833+/-0.1833$ & $0.000968+/-0.001071$ \\
\hline & & & Rehoboth & 11 & 7 & 6 & $0.7273+/-0.1444$ & $0.002839+/-0.002173$ \\
\hline & & & Manning & 1 & 0 & 1 & $1.0000+/-0.0000$ & $0.000000+/-0.000000$ \\
\hline & & & Nashville & - & - & - & - & - \\
\hline & & Corner Rise & Kukenthal & - & - & - & - & - \\
\hline & & & Goode & 8 & 2 & 3 & $0.6071+/-0.1640$ & $0.002100+/-0.001807$ \\
\hline & & & $\begin{array}{l}\text { Verrill } \\
\text { Milne- } \\
\text { Edwards }\end{array}$ & 8 & 1 & 3 & $0.4643+/-0.2000$ & $0.000547+/-0.000775$ \\
\hline & & & Lyman & 7 & 5 & 4 & $0.7143+/-0.1809$ & $0.003186+/-0.002513$ \\
\hline & & Muir & Muir & - & - & - & - & - \\
\hline
\end{tabular}




\section{Population Structure}

Parsimony network analysis of the $16 \mathrm{~S}$ datasets for A. clavigera, O. oedipus, and $O$. chelys, as well as of the COI dataset for O. oedipus were all characterized by a starlike phylogeny with a single haplotype in the center, which was identified as the "ancestral-" or "root-haplotype" (Castelloe and Templeton 1994; Crandall and Templeton 1993). The root haplotype was numerically dominant in each of these cases. For $A$. clavigera, a total of 22 haplotypes were found with the root-haplotype being shared by 9 of the 12 seamount peak populations sampled (Figure 3). A similar pattern was shown for two molecular makers for O. oedipus. For $16 \mathrm{~S}$, the root haplotype of O. oedipus was the most abundant haplotype out of a total of 5 haplotypes and was present on 11 out 12 seamount peak populations. For $O$. chelys, there were a total of 15 haplotypes of $16 \mathrm{~S}$ and the root-haplotype was found on 5 out of 8 seamount peak populations (Figure 5 and Figure 8).

The haplotype network obtained from 16S sequences of $O$. abyssalis showed 15 haplotypes, the two most common were centrally located within the network. The roothaplotype was the most abundant haplotype and distributed on all six seamount populations included in the $O$. abyssalis dataset (Figure 7). This pattern is similar to the one observed for the $\mathrm{COI}$ dataset of $O$. oedipus. There were a total of nine haplotypes of COI, with the two dominant haplotypes centrally located and the root-haplotype found on 4 out of 10 seamount peak populations (Figure 6).

The haplotype network obtained from the COI dataset of $A$. clavigera did not show the typical star-phylogeny found in the other samples. There were 19 haplotypes and almost all were unique. The root-haplotype occurred only twice, but was centrally located. The other haplotypes often differed by several nucleotide substitutions (Figure 4).

Geographical representation of these data give indications of the distribution of the different haplotypes for the four different species (Figure 9 - Figure 14). Haplotype 2 was the dominant $16 \mathrm{~S}$ haplotype in $A$. clavigera and was distributed across both the New 
England and Corner Rise seamounts, being absent only from Balanus seamount. It also accounted for at least half or more of the haplotypes found in each location (Figure 9). All the haplotypes from the Corner Rise seamounts were shared by populations within the New England seamount chain. There were a total of 12 unique haplotypes in the New England seamount chain and one haplotype found only on Muir. Many of the unique haplotypes were found in only a single individual, one on Bear, one on Picket, one on Balanus, one on Kelvin, five on Kelvin, four on Rehoboth, one on Nashville, and one on Muir. Manning was the most extensively sampled seamount and had 11 of the 22 haplotypes (Figure 9).

Every haplotype of COI for A. clavigera was unique, except for Haplotype 12, which was found on Manning and Rehoboth seamounts (Figure 10).

There was one dominant $16 \mathrm{~S}$ haplotype for $O$. oedipus, Haplotype 1 which was found across the New England and Corner Rise seamounts (Figure 11). Haplotype 2 was found only on Bear seamount and Haplotype 5 was found only on Goode seamount, while Haplotypes 3 and 4 were found only on Rehoboth.

COI was variable for $O$. oedipus. Haplotypes 1,2, 7 and 9 were found across both seamount regions but the remaining six were found only either on the Corner Rise or on the New England seamounts (Figure 12).

Haplotypes 2 and 7 were the most dominant 16S haplotypes for $O$. abyssalis and they were distributed across the New England and Corner Rise seamounts. Haplotypes 1 and 15 were found only in the Corner Rise populations while Haplotypes 8, 9, 11, 12, 13, and 14, were found only in the New England seamount chain (Figure 13).

Haplotype 5 was the dominant $16 \mathrm{~S}$ haplotype for the $O$. chelys dataset and it was distributed across the New England and Corner Rise seamounts. Haplotypes 6, 7, 8, and 9 were found only in the Corner Rise while Haplotypes 1, 2, 10, 11, 12, 13, 14, and 15 were found only in the New England seamount chain (Figure 14). 


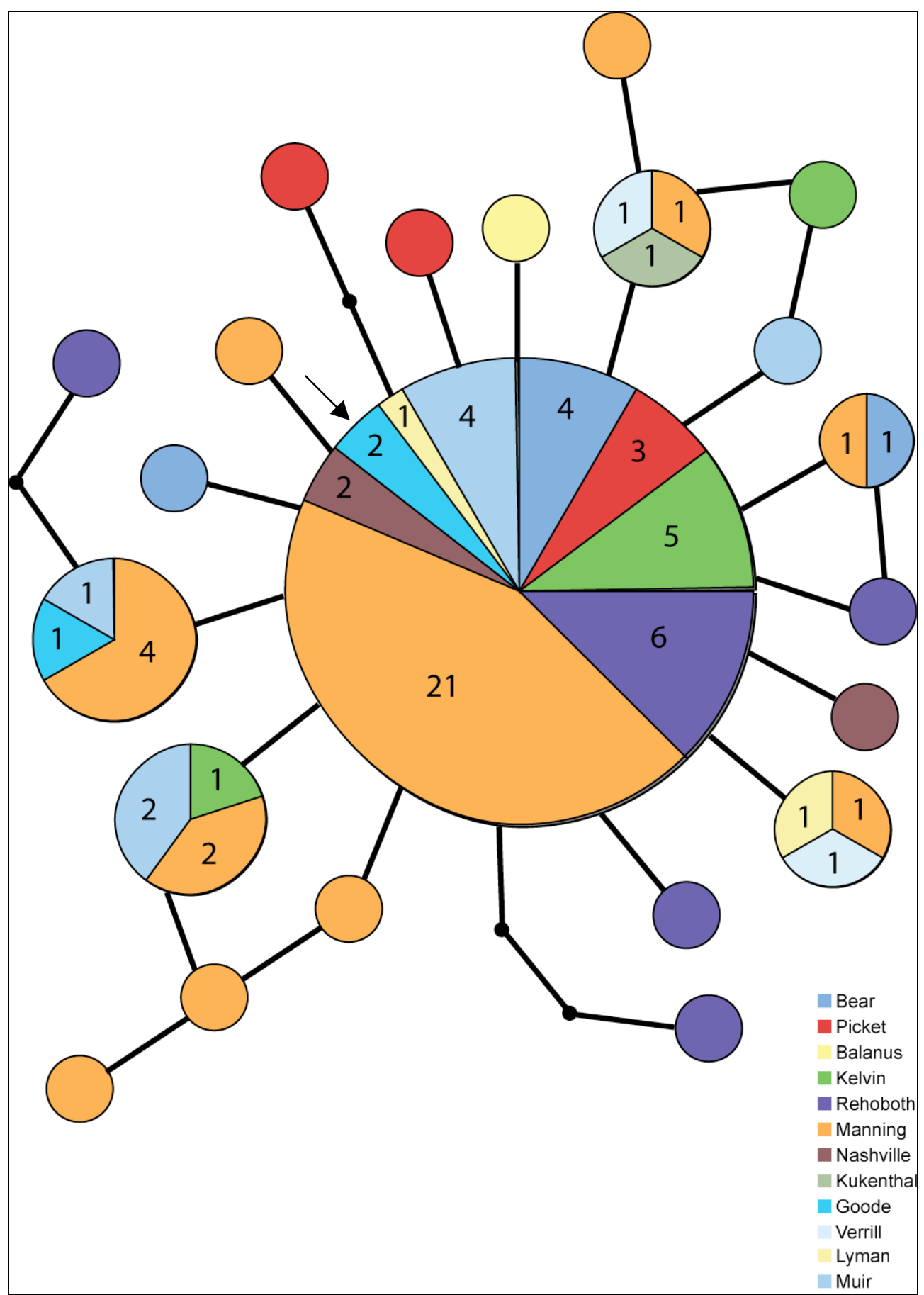

Figure 3. TCS haplotype network for $A$. clavigera $16 S$ sequences. Circles represent the different haplotypes observed, with circle areas proportional to the number of individuals possessing that haplotype. Haplotypes shared among geographic locations are further broken down into pie graphs. Connecting lines represent 1 base pair difference and small black circles represent interior node haplotypes not present in the samples. The ancestral haplotype is marked with an arrow. 


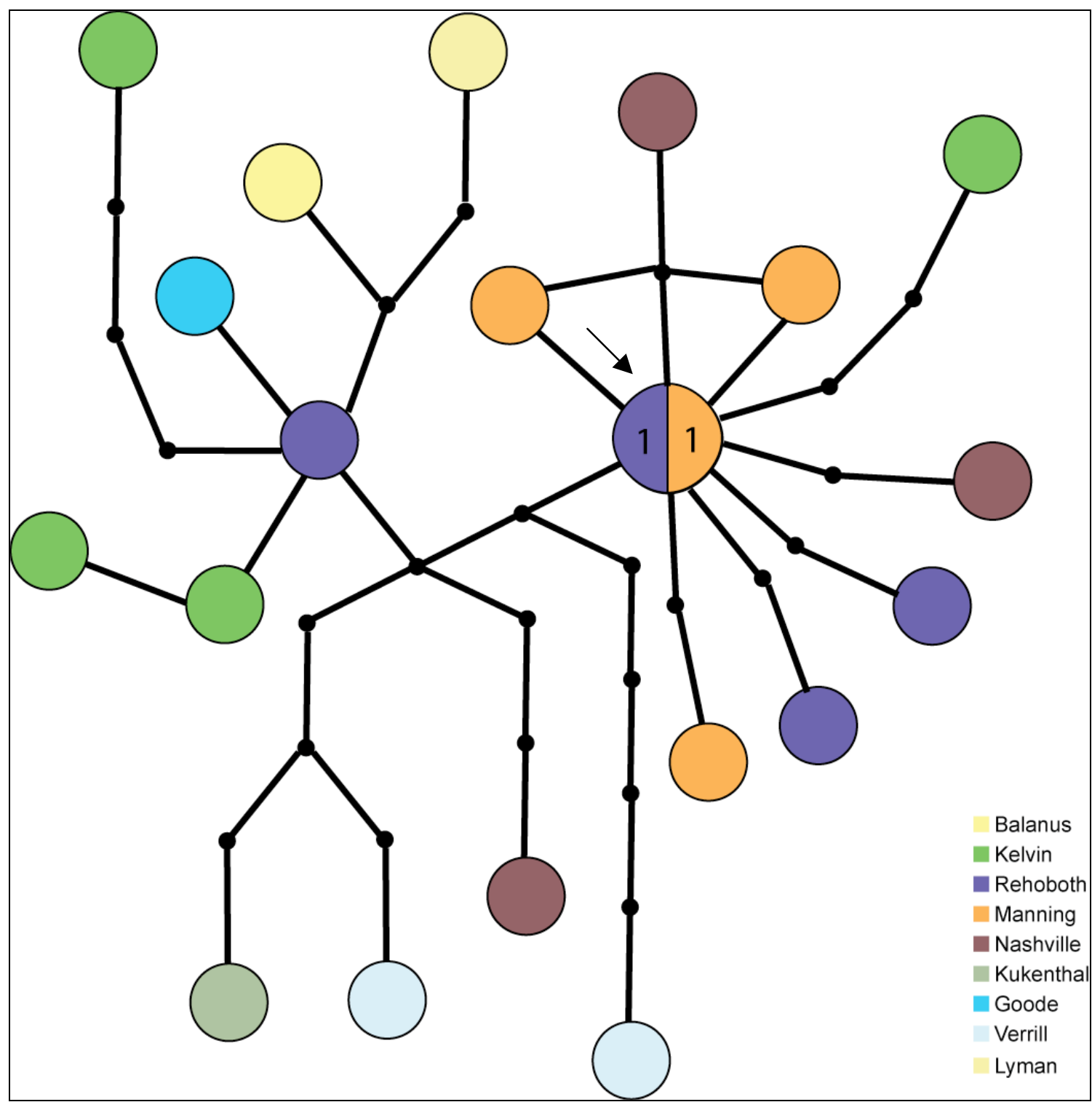

Figure 4. TCS haplotype network for $A$. clavigera COI sequences. Circles represent the different haplotypes observed, with circle areas proportional to the number of individuals possessing that haplotype. Haplotypes shared among geographic locations are further broken down into pie graphs. Connecting lines represent 1 base pair difference and small black circles represent interior node haplotypes not present in the samples. The ancestral haplotype is marked with an arrow. 


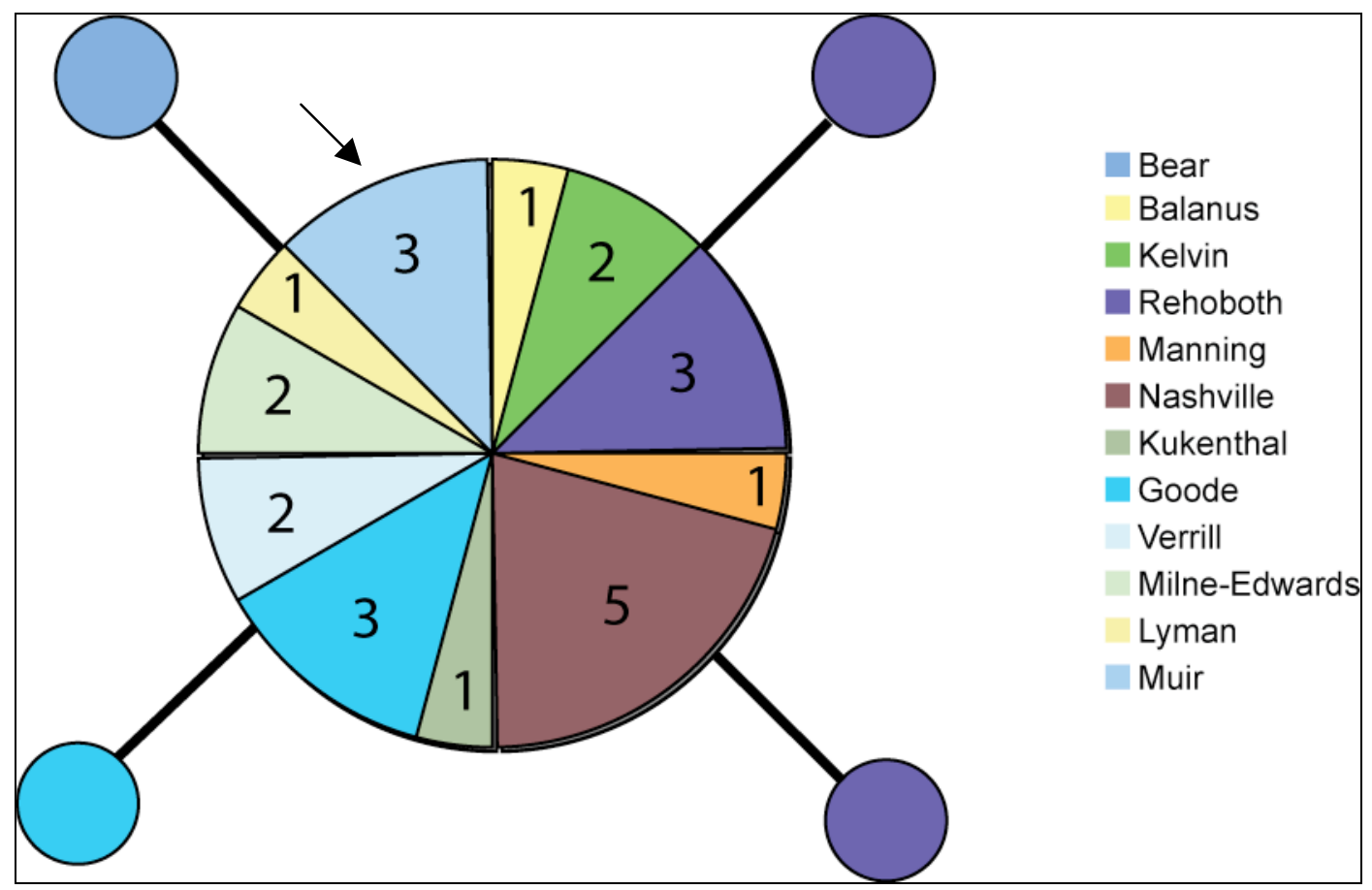

Figure 5. TCS haplotype network for O. oedipus 16S sequences. Circles represent the different haplotypes observed, with circle areas proportional to the number of individuals possessing that haplotype. Haplotypes shared among geographic locations are further broken down into pie graphs. Connecting lines represent 1 base pair difference and small black circles represent interior node haplotypes not present in the samples. The ancestral haplotype is marked with an arrow. 


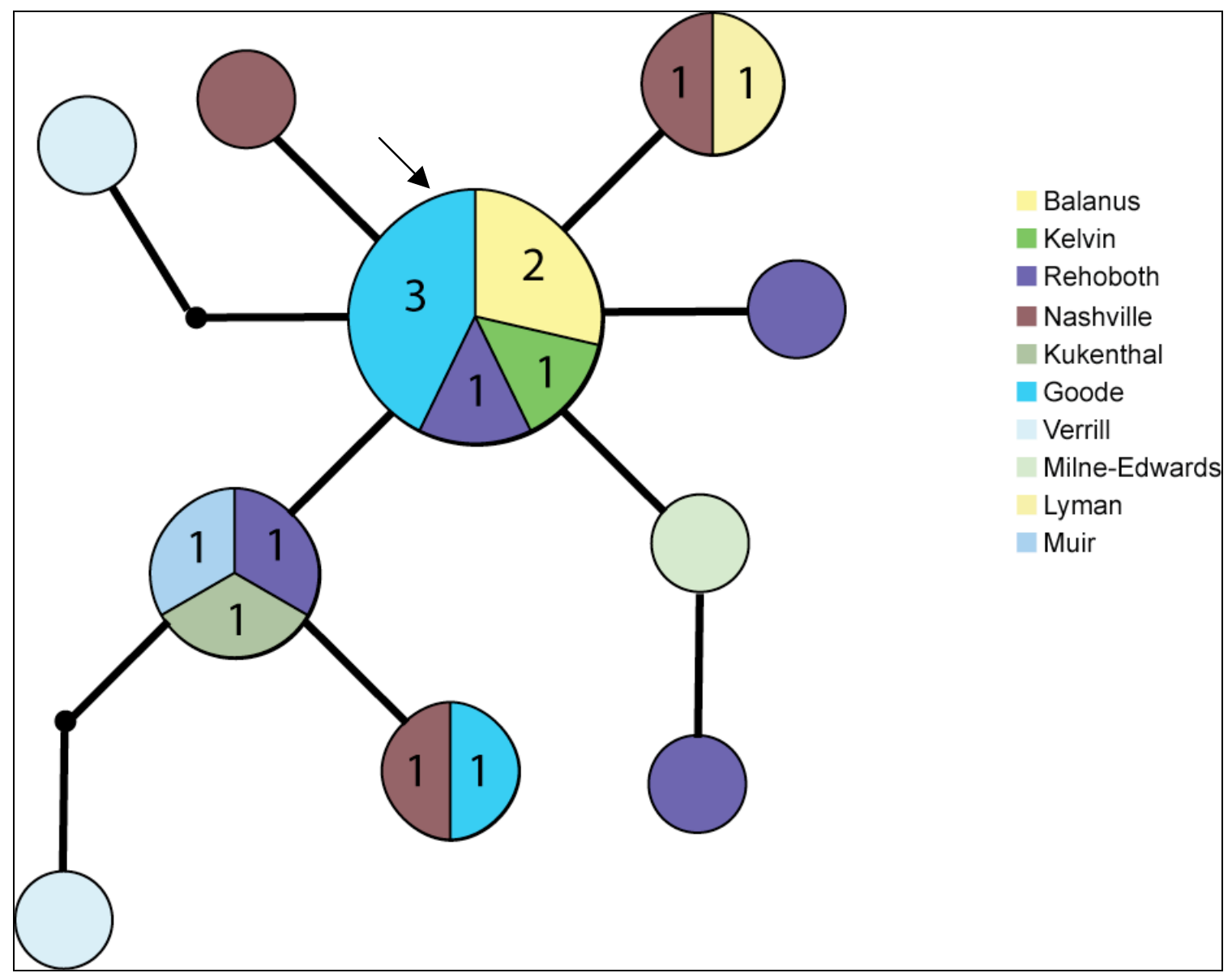

Figure 6. TCS haplotype network for O. oedipus COI sequences. Circles represent the different haplotypes observed, with circle areas proportional to the number of individuals possessing that haplotype. Haplotypes shared among geographic locations are further broken down into pie graphs. Connecting lines represent 1 base pair difference and small black circles represent interior node haplotypes not present in the samples. The ancestral haplotype is marked with an arrow. 


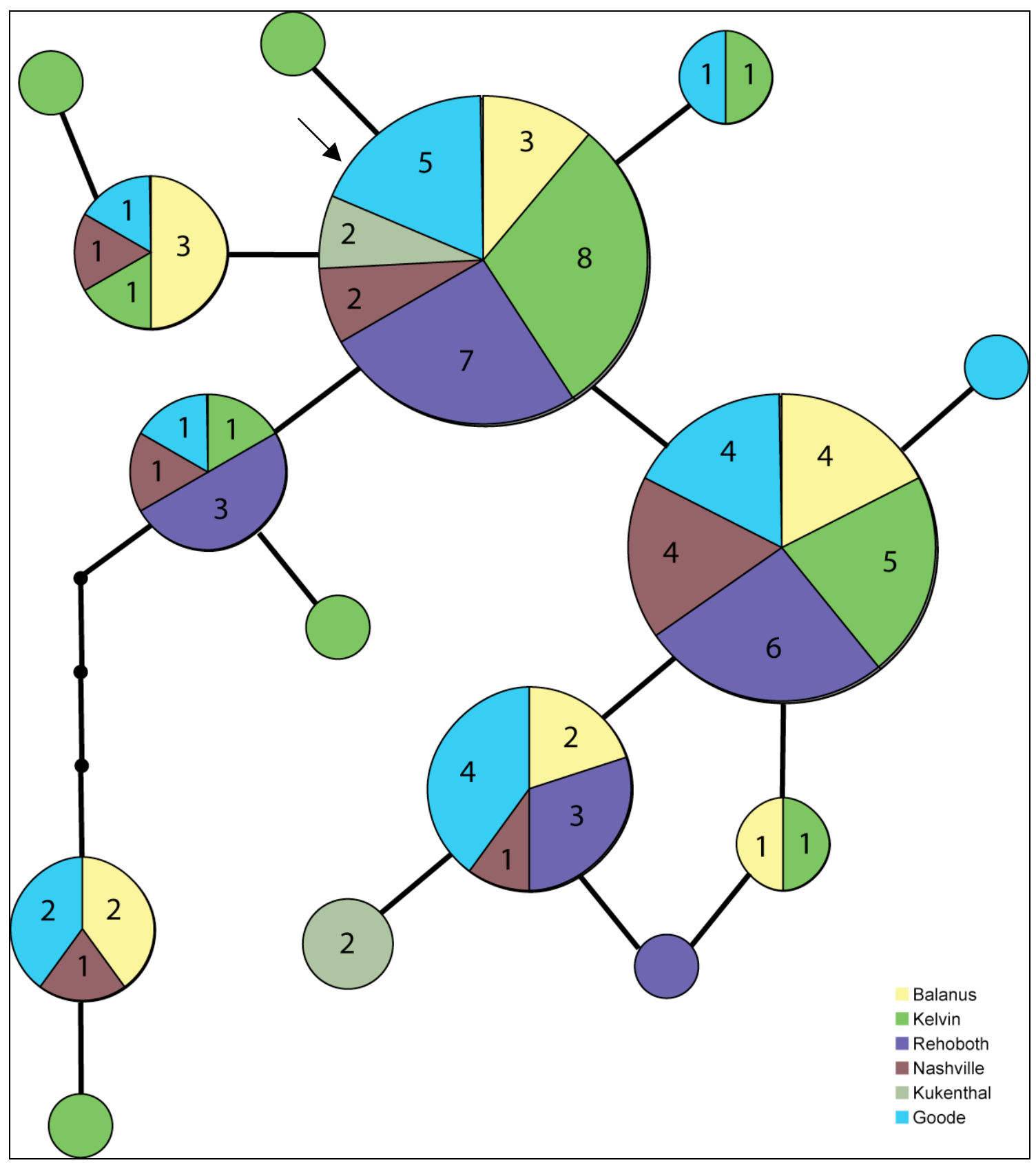

Figure 7. TCS haplotype network for $O$. abyssalis $16 \mathrm{~S}$ sequences. Circles represent the different haplotypes observed, with circle areas proportional to the number of individuals possessing that haplotype. Haplotypes shared among geographic locations are further broken down into pie graphs. Connecting lines represent 1 base pair difference and small black circles represent interior node haplotypes not present in the samples. The ancestral haplotype is marked with an arrow. 


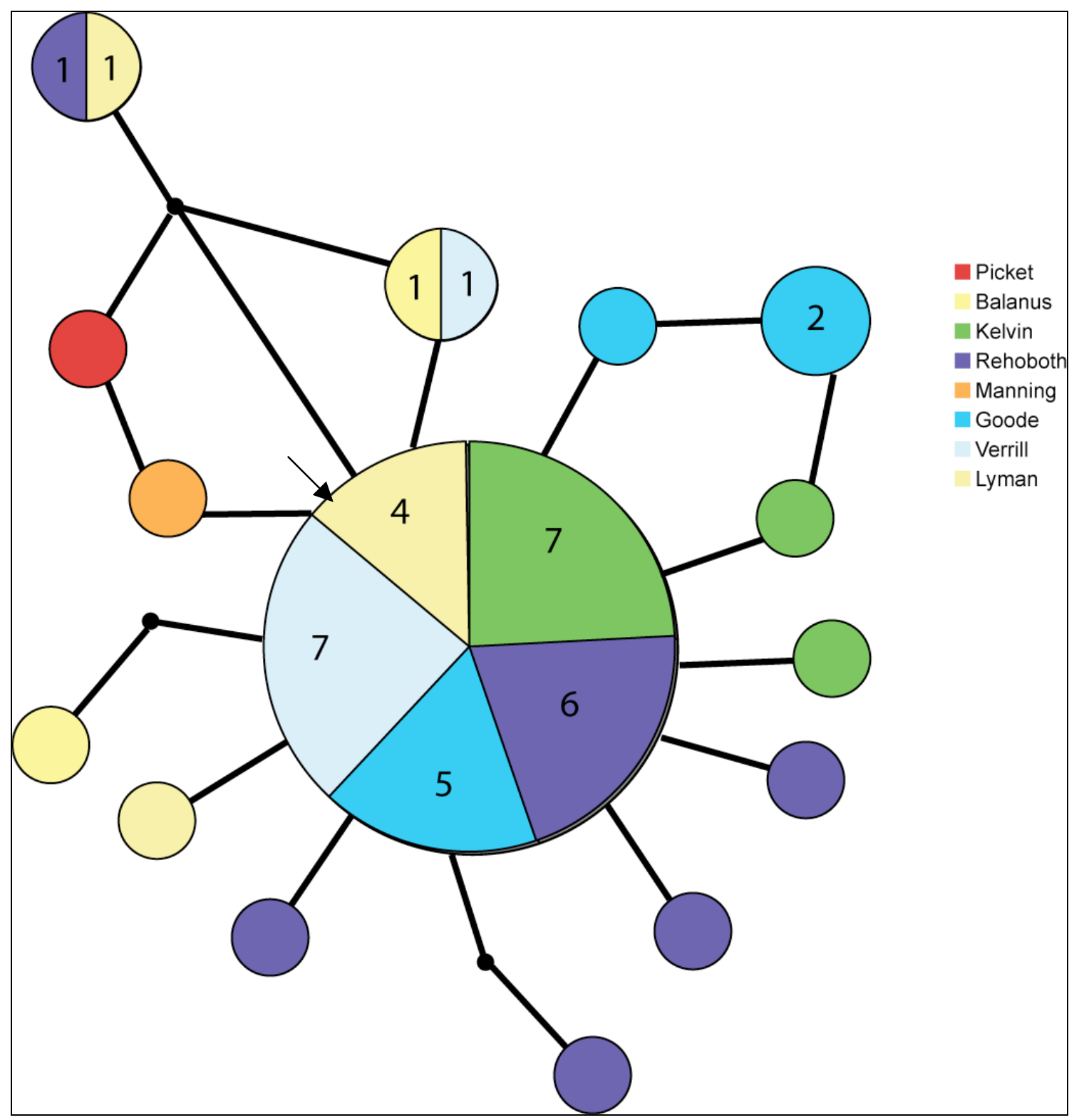

Figure 8. TCS haplotype network for $O$. chelys $16 \mathrm{~S}$ sequences. Circles represent the different haplotypes observed, with circle areas proportional to the number of individuals possessing that haplotype. Haplotypes shared among geographic locations are further broken down into pie graphs. Connecting lines represent 1 base pair difference and small black circles represent interior node haplotypes not present in the samples. The ancestral haplotype is marked with an arrow. 


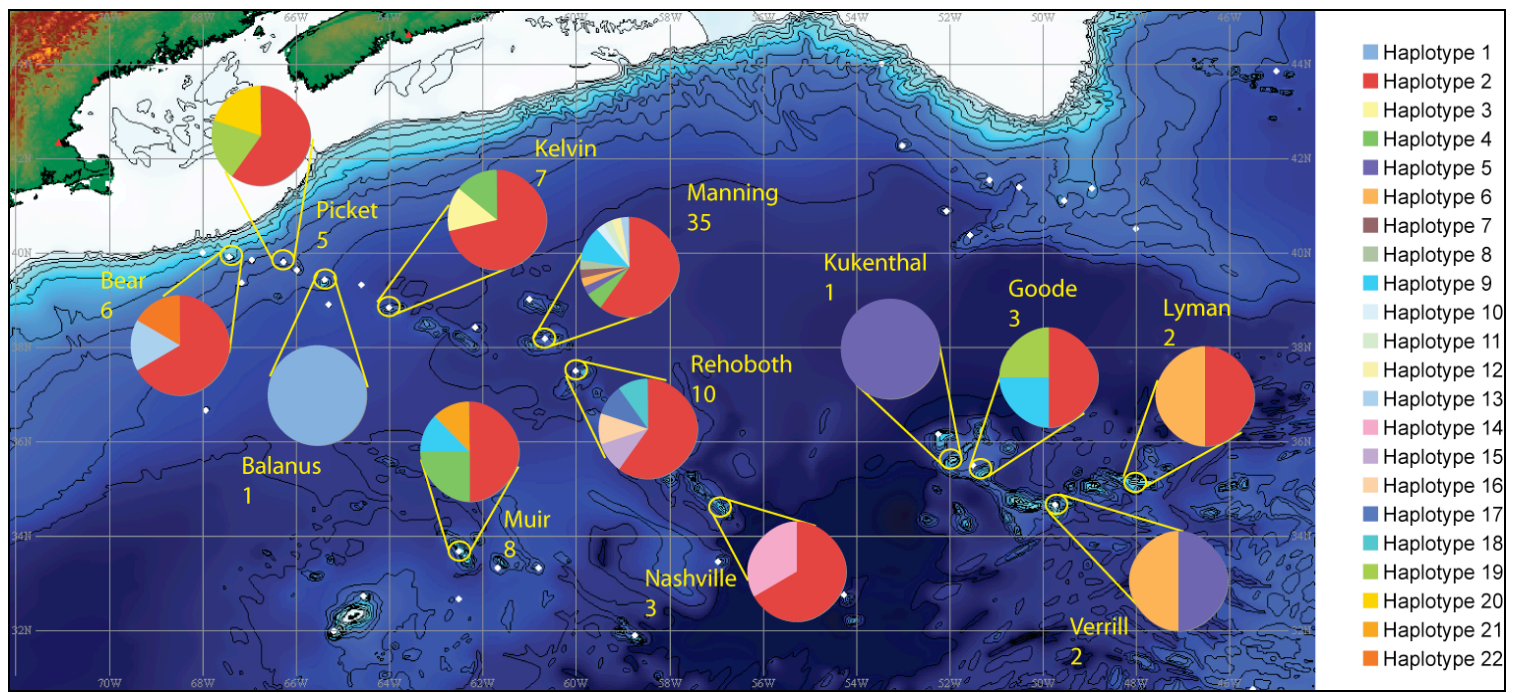

Figure 9. Haplotype map for $16 \mathrm{~S}$ sequences of $A$. clavigera. Pie graphs indicate haplotypic composition of each location and numbers indicate total number of samples from each location. Haplotype 2 is the ancestral haplotype.

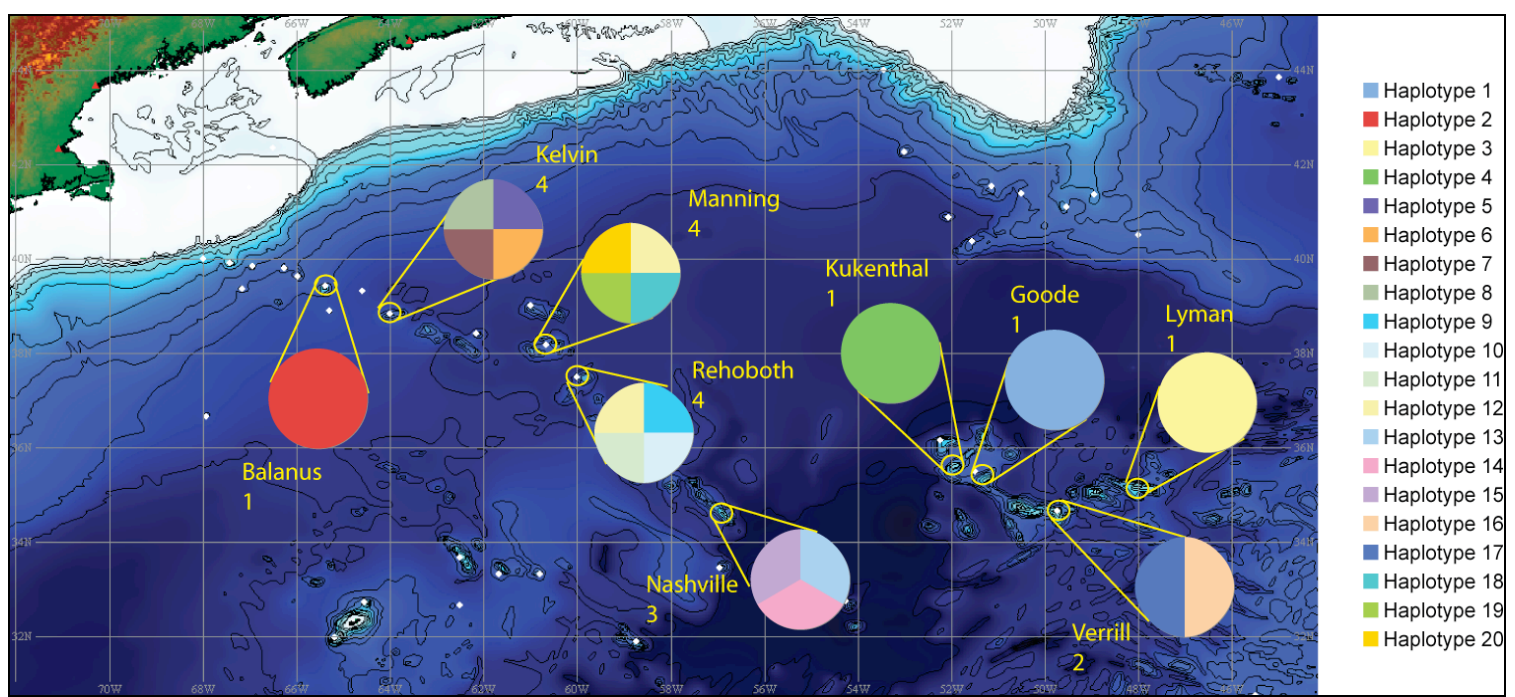

Figure 10. Haplotype map for COI sequences of $A$. clavigera. Pie graphs indicate haplotypic composition of each location and numbers indicate total number of samples from each location. Haplotype 12 is the ancestral haplotype. 


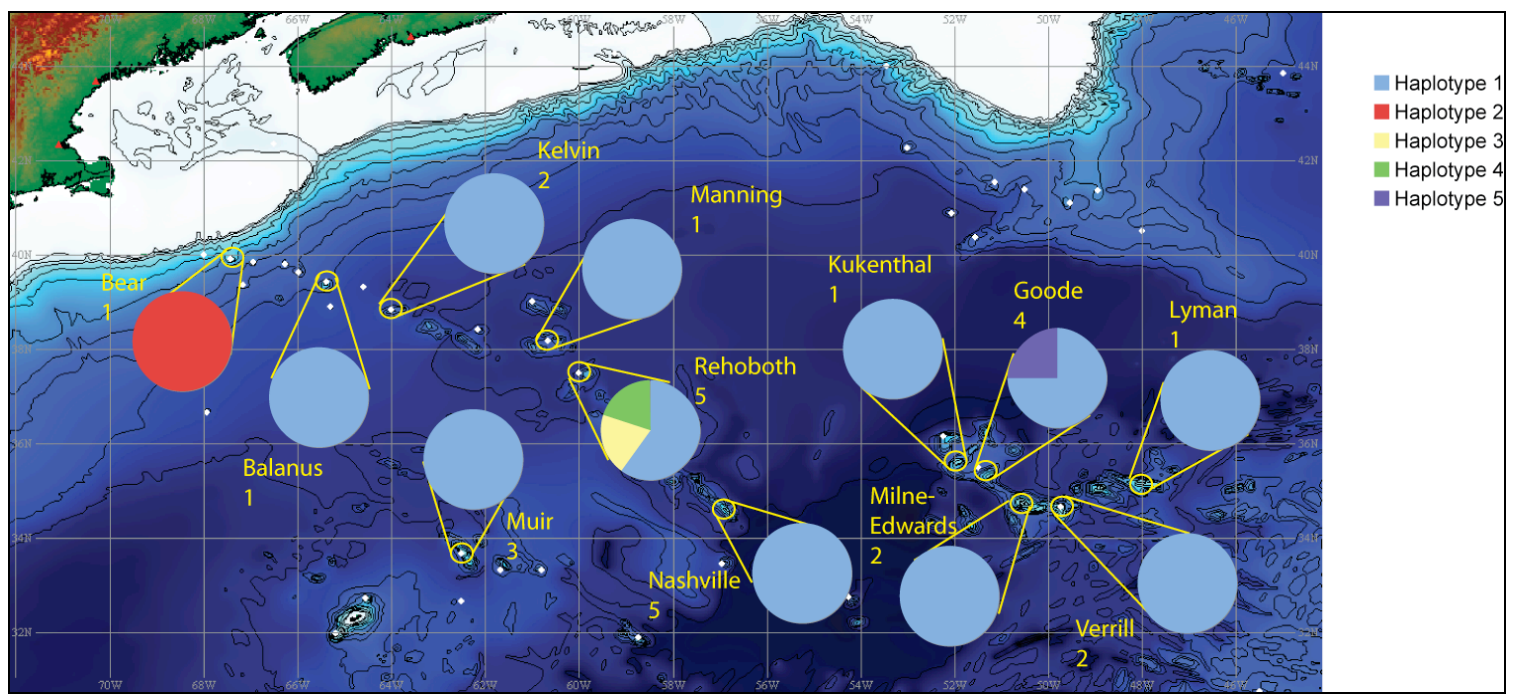

Figure 11. Haplotype map for $16 \mathrm{~S}$ sequences of $O$. oedipus. Pie graphs indicate haplotypic composition of each location and numbers indicate total number of samples from each location. Haplotype 1 is the ancestral haplotype.

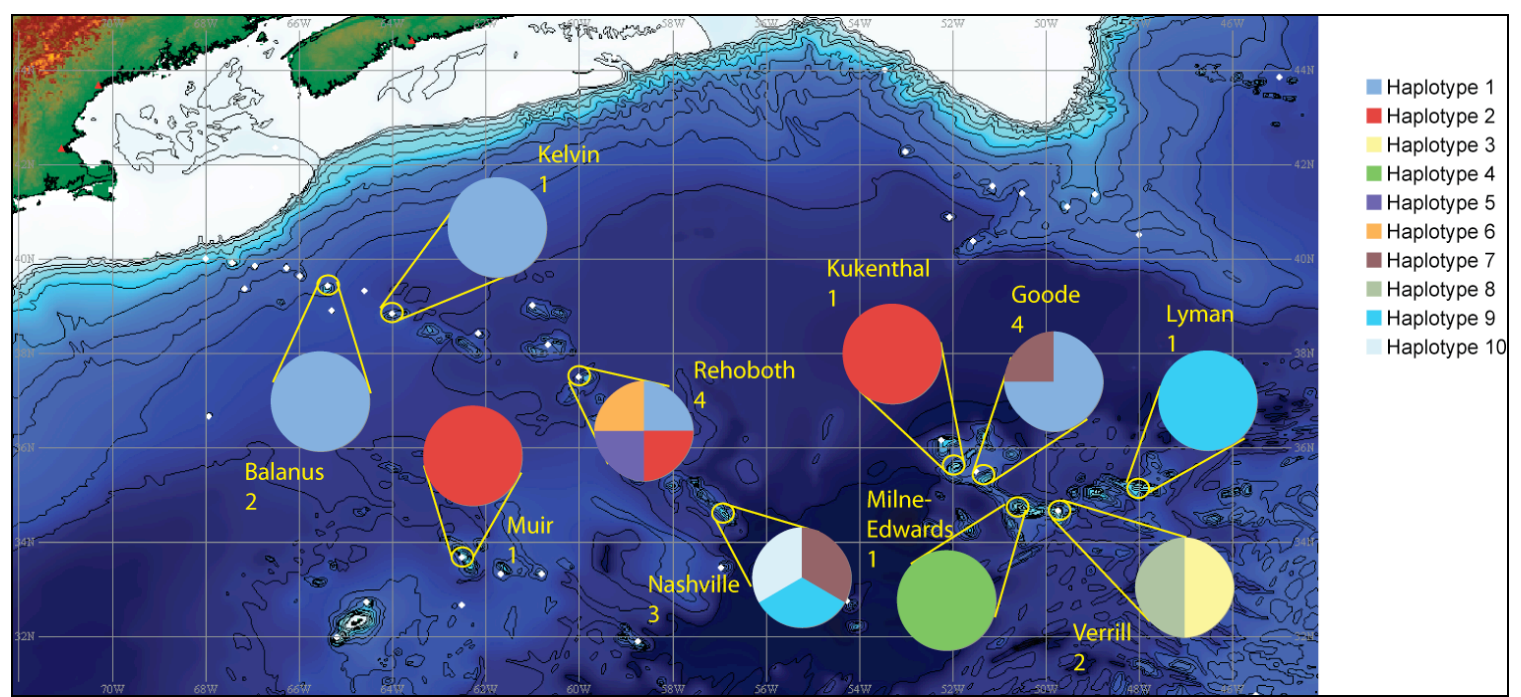

Figure 12. Haplotype map for COI sequences of $O$. oedipus. Pie graphs indicate haplotypic composition of each location and numbers indicate total number of samples from each location. Haplotype 1 is the ancestral haplotype. 


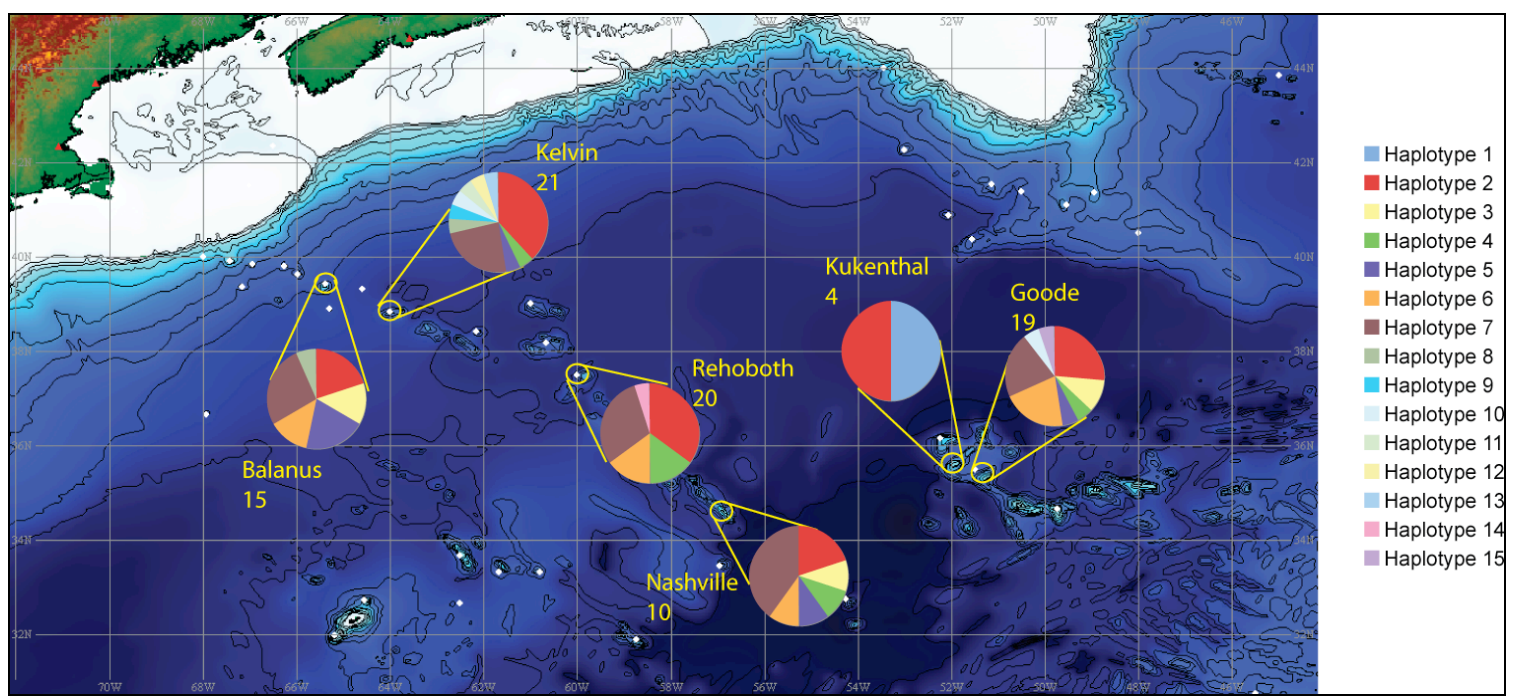

Figure 13. Haplotype map for $16 \mathrm{~S}$ sequences of $O$. abyssalis. Pie graphs indicate haplotypic composition of each location and numbers indicate total number of samples from each location. Haplotype 2 is the ancestral haplotype.

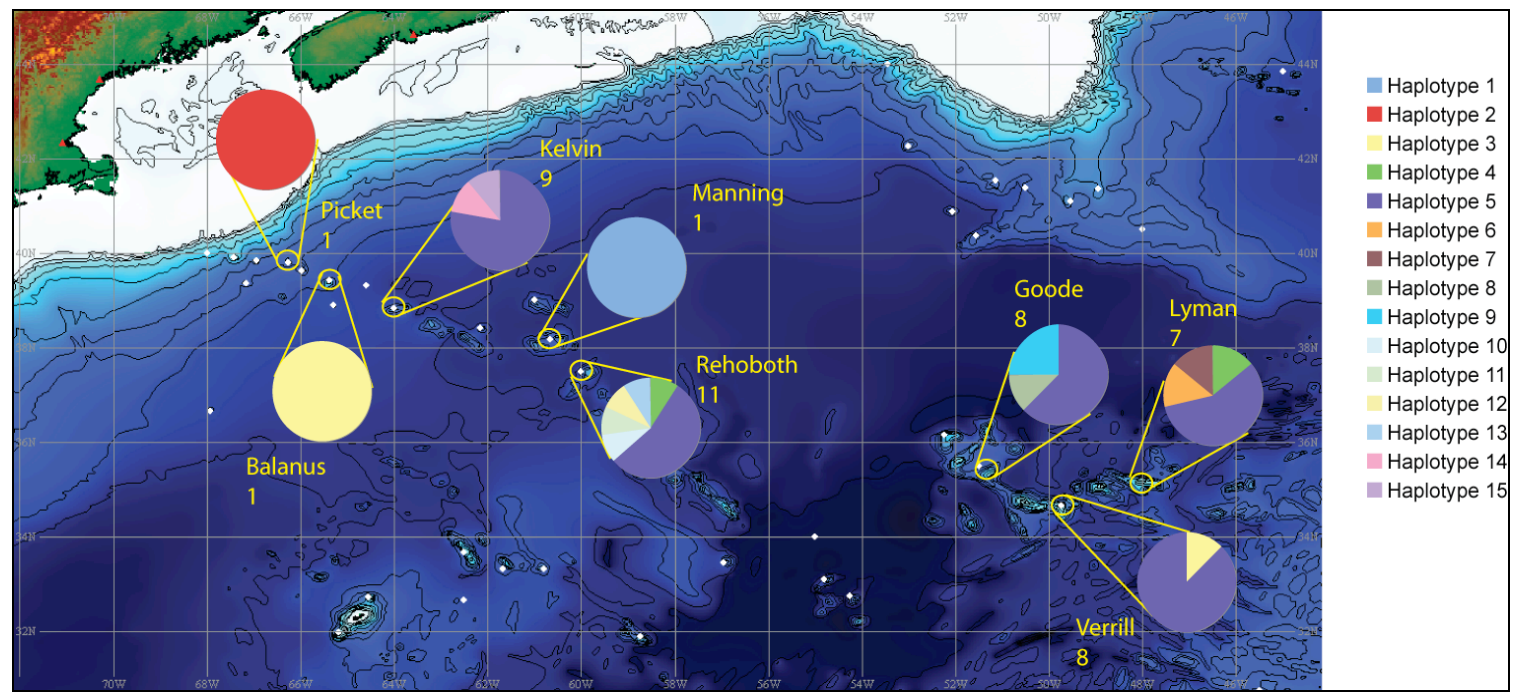

Figure 14. Haplotype map for $16 \mathrm{~S}$ sequences of $O$. chelys. Pie graphs indicate haplotypic composition of each location and numbers indicate total number of samples from each bcation. Haplotype 5 is the ancestral haplotype.

The AMOVA analyses for the $A$. clavigera $16 \mathrm{~S}$ dataset, all three $O$. oedipus datasets, and the $O$. abyssalis $16 \mathrm{~S}$ dataset showed no significant differences at the hierarchical groups tested. Slight significant differences were detected at some hierarchical levels tested for $A$. clavigera in the COI dataset and for the $O$. chelys $16 \mathrm{~S}$ dataset. Within the $A$. clavigera $\mathrm{COI}$ fragment 1 dataset differences within populations 
explained the largest amount of variance for $16 \mathrm{~S}(\mathrm{p}<0.05)$, followed by differences among populations within regional groups ( $\mathrm{p}<0.01$; Table 4). The AMOVA analyses for $O$. chelys also showed significant differences within populations, which explained the largest amount of total variance for $16 \mathrm{~S}(87 \%, \mathrm{p}<0.05)$ followed by differences among populations within regional groups $(19 \%, \mathrm{p}<0.05$; Table 4$)$.

The Mantel test showed a slightly significant correlation for the O. oedipus $16 \mathrm{~S}$ dataset supporting isolation by distance for this species (Table 5).

Table 4. Hierarchical AMOVA for seamount populations of four ophiuroid species using $16 \mathrm{~S}$ and COI. Significant values of $P<0.05$ are in bold.

\begin{tabular}{|c|c|c|c|c|c|c|c|}
\hline Species & Gene & Source of variation & d.f. & SS & $\begin{array}{l}\text { Variance } \\
\text { Components } \\
\end{array}$ & $\begin{array}{l}\% \\
\text { Variation } \\
\end{array}$ & $\mathrm{P}$ value \\
\hline \multirow[t]{6}{*}{ A. clavigera } & $16 S$ & Among Regional groups & 2 & 0.917 & 0.00118 & 0.27 & 0.38584 \\
\hline & & regional groups & 9 & 3.97 & 0.00125 & 0.29 & 0.37881 \\
\hline & & Within populations & 71 & 30.814 & 0.434 & 99.44 & 0.42812 \\
\hline & $\mathrm{COI}$ & Among Regional groups & 1 & 5.812 & 0.36716 & 12.08 & 0.05653 \\
\hline & & regional groups & 7 & 22.014 & 0.36906 & 12.14 & 0.00743 \\
\hline & & Within populations & 12 & 27.632 & 2.30264 & 75.77 & 0.01931 \\
\hline \multirow[t]{6}{*}{ O. oedipus } & $16 S$ & Among Regional groups & 2 & 0.157 & -0.00908 & -6.5 & 0.58162 \\
\hline & & $\begin{array}{l}\text { Among populations witnin } \\
\text { regional groups }\end{array}$ & 9 & 1.352 & 0.00145 & 1.04 & 0.27859 \\
\hline & & Within populations & 16 & 2.356 & 0.14725 & 105.46 & 0.57380 \\
\hline & $\mathrm{COI}$ & Among Regional groups & 2 & 1.029 & -0.05125 & -6.2 & 0.84733 \\
\hline & & regional groups & 7 & 5.563 & -0.09077 & -10.97 & 0.86980 \\
\hline & & Within populations & 10 & 9.691 & 0.96906 & 117.17 & 0.83198 \\
\hline \multirow[t]{3}{*}{ O. abyssalis } & $16 S$ & Among Regional groups & 1 & 0.842 & -0.00246 & -0.26 & 0.54252 \\
\hline & & regional groups & 4 & 3.724 & -0.00185 & -0.19 & 0.62463 \\
\hline & & Within populations & 83 & 79.39 & 0.95651 & 100.45 & 0.46921 \\
\hline \multirow[t]{3}{*}{ O. chelys } & $16 S$ & Among Regional groups & 1 & 0.503 & -0.03258 & -6.44 & 0.64907 \\
\hline & & regional groups & 6 & 5.517 & 0.0978 & 19.34 & 0.03128 \\
\hline & & Within populations & 38 & 16.734 & 0.44037 & 87.1 & 0.01857 \\
\hline
\end{tabular}

Bold values indicate $\mathrm{P}<0.05$

Table 5. Mantel correlation test between genetic distances (Kimura-2-Parameter) matrix for 4 species of ophiuroids for $16 \mathrm{~S}$ and COI and geographical distances between seamount peaks.

\begin{tabular}{llrr}
\hline Species & Gene & Correlation coefficient & P value \\
\hline A. clavigera & $16 S$ & 0.111611 & 0.192 \\
& COI & 0.149869 & 0.154 \\
\hline O. oedipus & $16 S$ & 0.289105 & $\mathbf{0 . 0 1 1}$ \\
& COI & 0.155791 & 0.119 \\
O. abyssalis & $16 S$ & 0.110715 & 0.312 \\
\hline O. chelys & $16 S$ & -0.102378 & 0.788 \\
\hline
\end{tabular}

Bold values indicate $\mathrm{P}<0.05$ 


\section{Test of Neutrality}

Tajima's D was found to be negative and significant for 3 populations for two different species of ophiuoroids. The population at Rehoboth had significant negative values of Tajima's D for both the $16 \mathrm{~S}$ dataset of $A$. clavigera and $O$. chelys $(\mathrm{p}<$ 0.01Table 6). Tajima's D for the Manning population in the $16 \mathrm{~S}$ dataset of $A$. clavigera and the Lyman population in the $16 \mathrm{~S}$ O. chelys dataset were also negative and slightly significant $(\mathrm{p}<0.5$; Table 6$)$.

The goodness-of-fit test to the model of sudden expansion could not be rejected for most seamount peak populations (Table 7). There was slight significance for the Muir population in the $16 \mathrm{~S}$ A. clavigera dataset $(\mathrm{p}=0.05)$ and higher significance for the Kukenthal population in the $O$. abyssalis $16 \mathrm{~S}$ dataset $(\mathrm{p}=0)$. This means that the model of recent population expansion can be rejected for these populations for these species. 
Table 6. Test of neutrality for each seamount peak population and significance for the four species of ophiuroids and each molecular marker dataset, estimated from 1000 replicates.

\begin{tabular}{|c|c|c|c|c|c|c|c|c|c|c|c|c|}
\hline \multirow[b]{3}{*}{$\begin{array}{l}\text { Seamount } \\
\text { peak }\end{array}$} & \multicolumn{4}{|c|}{ A. clavigera } & \multicolumn{4}{|c|}{ O. oedipus } & \multirow{2}{*}{\multicolumn{2}{|c|}{$\begin{array}{l}\text { O. abyssalis } \\
\text { 16S }\end{array}$}} & \multirow{2}{*}{\multicolumn{2}{|c|}{$\begin{array}{l}\text { O. chelys } \\
16 \mathrm{~S}\end{array}$}} \\
\hline & $16 \mathrm{~S}$ & & $\mathrm{COI}$ & & $16 S$ & & $\mathrm{COI}$ & & & & & \\
\hline & Tajima's D & $\mathrm{P}$ value & Tajima's D & $P$ value & Tajima's D & $P$ value & Tajima's D & $\begin{array}{c}\mathrm{P} \\
\text { value }\end{array}$ & Tajima's D & $\begin{array}{c}\mathrm{P} \\
\text { value }\end{array}$ & Tajima's D & $\begin{array}{c}\mathrm{P} \\
\text { value }\end{array}$ \\
\hline Bear & -1.13197 & 0.151 & - & - & 0 & 1 & - & - & - & - & - & - \\
\hline Picket & -1.04849 & 0.146 & - & - & - & - & - & - & - & - & 0 & 1 \\
\hline Balanus & 0 & 1 & 0 & 1 & 0 & 1 & 0 & 1 & -0.03332 & 0.525 & 0 & 1 \\
\hline Kelvin & -1.23716 & 0.113 & -0.58365 & 0.403 & 0 & 1 & 0 & 1 & -1.5383 & 0.056 & -1.3624 & 0.09 \\
\hline Rehoboth & -1.79631 & 0.009 & -0.81734 & 0.142 & -0.97256 & 0.201 & -0.78012 & 0.2 & 0.26729 & 0.619 & -1.89693 & 0.007 \\
\hline Manning & -1.70044 & 0.037 & -0.78012 & 0.199 & 0 & 1 & - & - & - & - & 0 & 1 \\
\hline Nashville & 0 & 0.987 & 30031065.1 & 1 & 0 & 1 & 0 & 0.824 & -0.65991 & 0.27 & - & - \\
\hline Kukenthal & 0 & 1 & 0 & 1 & 0 & 1 & 0 & 1 & 2.01187 & 0.973 & - & - \\
\hline Goode & 0 & 0.986 & 0 & 1 & -0.61237 & 0.362 & -0.7099 & 0.286 & -0.43045 & 0.401 & 0.93151 & 0.846 \\
\hline $\begin{array}{l}\text { Verrill } \\
\text { Milne- }\end{array}$ & 0 & 1 & 0 & 1 & 0 & 1 & 0 & 1 & - & - & -1.05482 & 0.222 \\
\hline Edwards & - & - & - & - & 0 & 1 & 0 & 1 & - & - & - & - \\
\hline Lyman & 0 & 1 & 0 & 1 & 0 & 1 & 0 & 1 & - & - & -1.48614 & 0.029 \\
\hline Muir & -0.81246 & 0.28 & - & - & 0 & 1 & 0 & 1 & - & - & - & - \\
\hline
\end{tabular}


Table 7. Test of goodness of fit to the model of sudden expansion, for each species and each molecular marker. $X$ indicates seamount peaks not included in that dataset. Dashes indicate populations where the variance of the mismatch distribution was too small for demographic parameters to be estimated.

\begin{tabular}{|c|c|c|c|c|c|c|c|}
\hline \multirow{2}{*}{$\begin{array}{l}\text { Test of goodness- } \\
\text { of-fit }\end{array}$} & \multirow{2}{*}{$\begin{array}{l}\text { Seamount } \\
\text { peak }\end{array}$} & \multicolumn{2}{|c|}{ A. clavigera } & \multicolumn{2}{|c|}{ O. oedipus } & \multirow{2}{*}{$\begin{array}{c}\text { O. abyssalis } \\
16 \mathrm{~S}\end{array}$} & \multirow{2}{*}{$\begin{array}{c}\text { O. chelys } \\
16 S\end{array}$} \\
\hline & & $16 S$ & $\mathrm{COI}$ & $16 S$ & $\mathrm{COI}$ & & \\
\hline $\begin{array}{l}\text { Sum of squared } \\
\text { deviation } \\
\mathrm{P} \text { (Sim. Ssd } \geq\end{array}$ & Bear & 0.0373003 & $X$ & - & $X$ & $x$ & $x$ \\
\hline obs. Ssd) & & 0.5 & $x$ & - & $x$ & $x$ & $\mathrm{X}$ \\
\hline $\begin{array}{l}\text { Sum of squared } \\
\text { deviation } \\
\mathrm{P}(\text { Sim. Ssd } \geq\end{array}$ & Picket & 0.00628516 & $X$ & $X$ & $X$ & $x$ & - \\
\hline $\begin{array}{l}\text { obs. Ssd) } \\
\text { Sum of squared }\end{array}$ & & 0.8 & $x$ & $x$ & $x$ & $x$ & - \\
\hline $\begin{array}{l}\text { deviation } \\
\mathrm{P}(\text { Sim. Ssd } \geq\end{array}$ & Balanus & - & - & - & - & 0.02916628 & - \\
\hline obs. Ssd) & & - & - & - & - & 0.2 & - \\
\hline $\begin{array}{l}\text { Sum of squared } \\
\text { deviation } \\
\text { P(Sim. Ssd } \geq\end{array}$ & Kelvin & 0.00966579 & 0.05720079 & - & - & 0.00905134 & 0.00869826 \\
\hline $\begin{array}{l}\text { obs. Ssd) } \\
\text { Sum of squared }\end{array}$ & & 0.85 & 0.85 & - & - & 0.5 & 0.7 \\
\hline $\begin{array}{l}\text { deviation } \\
\mathrm{P}(\text { Sim. Ssd } \geq\end{array}$ & Rehoboth & 0.04667542 & 0.07353228 & 0.06517773 & 0.04206112 & 0.01078693 & 0.00209462 \\
\hline obs. Ssd) & & 0.65 & 0.6 & 0.2 & 0.65 & 0.4 & 0.85 \\
\hline $\begin{array}{l}\text { Sum of squared } \\
\text { deviation } \\
\text { P(Sim. Ssd } \geq\end{array}$ & Manning & 0.00120145 & 0.04206112 & - & $X$ & $x$ & - \\
\hline obs. Ssd) & & 0.95 & 0.6 & - & $x$ & $x$ & - \\
\hline $\begin{array}{l}\text { Sum of squared } \\
\text { deviation } \\
P(\text { Sim. Ssd } \geq\end{array}$ & Nashville & 0.08981407 & 0.17196414 & - & 0.23126894 & 0.02274179 & $X$ \\
\hline $\begin{array}{l}\text { obs. Ssd) } \\
\text { Sum of squared }\end{array}$ & & 0.45 & 0.45 & - & 0.2 & 0.3 & $x$ \\
\hline $\begin{array}{l}\text { Sum of squared } \\
\text { deviation } \\
P(\text { Sim. Ssd } \geq\end{array}$ & Kukenthal & - & - & - & - & 0.33436626 & $X$ \\
\hline obs. Ssd) & & - & - & - & - & 0 & $x$ \\
\hline $\begin{array}{l}\text { Sum of squared } \\
\text { deviation } \\
\text { P(Sim. Ssd } \geq\end{array}$ & Goode & 0.08981407 & - & - & 0.18925158 & 0.017331 & 0.02952579 \\
\hline obs. Ssd) & & 0.6 & - & - & 0.25 & 0.25 & 0.6 \\
\hline $\begin{array}{l}\text { Sum of squared } \\
\text { deviation }\end{array}$ & Verrill & - & - & - & - & $\mathrm{X}$ & 0.27913606 \\
\hline
\end{tabular}


Table 7. continued

\begin{tabular}{|c|c|c|c|c|c|c|c|}
\hline \multirow[b]{2}{*}{$\begin{array}{c}\text { Test of goodness- } \\
\text { of-fit }\end{array}$} & \multirow{2}{*}{$\begin{array}{c}\text { Seamount } \\
\text { peak }\end{array}$} & \multicolumn{2}{|c|}{ A. clavigera } & \multicolumn{2}{|c|}{ O. oedipus } & \multirow{2}{*}{$\begin{array}{c}\text { O. abyssalis } \\
16 \mathrm{~S}\end{array}$} & \multirow{2}{*}{$\begin{array}{c}\text { O. chelys } \\
16 \mathrm{~S}\end{array}$} \\
\hline & & $16 S$ & $\mathrm{COI}$ & $16 S$ & $\mathrm{COI}$ & & \\
\hline $\begin{array}{l}\text { Sum of squared } \\
\text { deviation } \\
P(\operatorname{Sim} S s d \geq\end{array}$ & $\begin{array}{l}\text { Milne- } \\
\text { Edwards }\end{array}$ & $x$ & $X$ & - & - & $x$ & $x$ \\
\hline $\begin{array}{l}P(\text { Sim. Ssd } \geq \\
\text { obs. Ssd }) \\
\text { Sum of squared }\end{array}$ & & $x$ & $x$ & - & - & $x$ & $x$ \\
\hline & Lyman & - & - & - & - & $x$ & 0.0344829 \\
\hline obs. Ssd) & & - & - & - & - & $x$ & 0.3 \\
\hline $\begin{array}{l}\text { Sum of squared } \\
\text { deviation } \\
\mathrm{P} \text { (Sim. Ssd } \geq\end{array}$ & Muir & 0.04814155 & $x$ & - & - & $x$ & $x$ \\
\hline obs. Ssd) & & 0.05 & $x$ & - & - & $x$ & $x$ \\
\hline
\end{tabular}




\section{Bayesian estimation of migration rates}

Estimates of the number of migrants per generation among the seamount populations were generally high, with typical values approximately 20 migrants/generation (Table 8 - Table 15). The migration of $O$. abyssalis was markedly lower however, at about 10 migrants/generation. These values are indicative of high migration between the populations, although certain populations had lower rates of migration, which varied dependent upon species and marker (Table 16). The Manning population appeared to experience relatively less migration compared to the other populations in the $16 \mathrm{~S}$ dataset for $A$. clavigera. The populations of Balanus, Nashville, and Rehoboth of $O$. oedipus had relatively less migrants per generation according to all molecular markers, while the Rehoboth population of $O$. abyssalis and the Goode and Verrill populations of $O$. chelys experienced less migration relative to the other populations. 
Table 8. Pairwise estimates of migration for the $16 \mathrm{~S}$ dataset of $A$. clavigera. The rows are the sending population and the columns are the receiving populations.

\begin{tabular}{|c|c|c|c|c|c|c|c|c|c|c|c|c|}
\hline & Bear & Picket & Balanus & Kelvin & Manning & Rehoboth & Nashville & Muir & Kukenthal & Goode & Verrill & Lyman \\
\hline Bear & - & 24.4 & 23.6 & 23.3 & 7.7 & 24.0 & 21.7 & 21.6 & 23.2 & 20.9 & 21.7 & 23.8 \\
\hline Picket & 22.5 & - & 21.1 & 22.9 & 5.3 & 24.0 & 20.1 & 22.8 & 23.1 & 19.3 & 21.5 & 22.3 \\
\hline Balanus & 25.3 & 22.1 & - & 23.8 & 6.7 & 24.2 & 24.2 & 25.7 & 22.5 & 22.7 & 23.3 & 26.2 \\
\hline Kelvin & 23.6 & 21.5 & 21.8 & - & 7.2 & 22.0 & 24.0 & 22.4 & 21.5 & 21.1 & 21.5 & 21.3 \\
\hline Manning & 4.6 & 4.4 & 4.5 & 4.7 & - & 4.4 & 4.5 & 4.5 & 4.3 & 4.2 & 4.6 & 4.3 \\
\hline Rehoboth & 24.6 & 23.7 & 21.5 & 24.2 & 5.0 & - & 24.0 & 21.8 & 23.0 & 21.6 & 23.4 & 23.9 \\
\hline Nashville & 26.2 & 26.4 & 23.7 & 23.7 & 7.1 & 24.4 & - & 20.5 & 25.7 & 23.0 & 24.4 & 23.3 \\
\hline Muir & 20.9 & 20.8 & 21.2 & 20.4 & 4.2 & 21.4 & 21.2 & - & 20.6 & 18.2 & 19.2 & 20.5 \\
\hline Kukenthal & 24.2 & 24.1 & 23.4 & 23.3 & 6.7 & 24.2 & 24.5 & 23.6 & - & 21.5 & 24.6 & 27.3 \\
\hline Goode & 21.7 & 24.8 & 22.7 & 24.0 & 7.8 & 23.5 & 22.5 & 22.2 & 22.1 & - & 22.9 & 24.9 \\
\hline Verrill & 19.0 & 20.7 & 20.2 & 20.8 & 5.7 & 22.3 & 21.8 & 23.1 & 21.1 & 18.4 & - & 22.8 \\
\hline Lyman & 22.6 & 23.1 & 24.3 & 23.3 & 6.8 & 23.6 & 26.5 & 22.7 & 24.2 & 22.0 & 25.1 & - \\
\hline
\end{tabular}

Table 9. Pairwise estimates of migration for the COI dataset of $A$. clavigera. The rows are the sending population and the columns are the receiving populations.

\begin{tabular}{|c|c|c|c|c|c|c|c|c|c|}
\hline & Balanus & Kelvin & Manning & Rehoboth & Nashville & Kukenthal & Goode & Verrill & Lyman \\
\hline Balanus & - & 21.4 & 22.1 & 18.9 & 25.5 & 22.9 & 23.0 & 24.5 & 24.1 \\
\hline Kelvin & 21.0 & - & 18.4 & 16.4 & 19.6 & 19.2 & 18.2 & 20.8 & 19.2 \\
\hline Manning & 21.1 & 19.9 & - & 17.3 & 22.9 & 21.0 & 20.1 & 21.7 & 20.8 \\
\hline Rehoboth & 20.1 & 17.4 & 17.8 & - & 19.2 & 19.3 & 18.0 & 19.6 & 19.0 \\
\hline Nashville & 24.5 & 22.0 & 21.4 & 18.2 & - & 22.8 & 21.6 & 24.4 & 23.2 \\
\hline Kukenthal & 24.3 & 20.3 & 21.6 & 18.8 & 23.5 & - & 22.5 & 25.3 & 23.0 \\
\hline Goode & 23.3 & 20.1 & 19.0 & 18.3 & 23.1 & 21.9 & - & 23.4 & 22.8 \\
\hline Verrill & 26.2 & 22.4 & 21.9 & 20.7 & 25.0 & 25.1 & 23.4 & - & 24.8 \\
\hline Lyman & 23.2 & 20.6 & 20.5 & 18.7 & 23.1 & 21.8 & 21.9 & 23.3 & - \\
\hline
\end{tabular}


Table 10. Pairwise estimates of migration for both $16 \mathrm{~S}$ and $\mathrm{COI}$ of $\boldsymbol{A}$. clavigera. The rows are the sending population and the columns are the receiving populations.

\begin{tabular}{|c|c|c|c|c|c|c|c|c|c|c|c|c|}
\hline & Bear & Picket & Balanus & Kelvin & Manning & Rehoboth & Nashville & Muir & Kukenthal & Goode & Verrill & Lyman \\
\hline Bear & - & 6.1 & 1.9 & 6.4 & 4.4 & 5.1 & 5.5 & 5.1 & 7.8 & 7.5 & 0.9 & 6.7 \\
\hline Picket & 31.4 & - & 18.5 & 22.6 & 19.9 & 9.1 & 23.1 & 4.6 & 5.8 & 31.3 & 20.4 & 12.7 \\
\hline Balanus & 5.8 & 8.9 & - & 12.6 & 15.9 & 4.4 & 17.0 & 10.5 & 8.2 & 15.4 & 14.1 & 19.7 \\
\hline Manning & 3.9 & 3.2 & 3.9 & 5.1 & - & 5.0 & 4.3 & 3.4 & 4.5 & 3.9 & 3.9 & 5.3 \\
\hline Rehoboth & 6.7 & 16.9 & 19.7 & 19.3 & 9.0 & - & 22.9 & 14.0 & 10.0 & 9.9 & 4.6 & 19.3 \\
\hline Nashville & 11.5 & 21.4 & 22.3 & 28.0 & 9.2 & 27.8 & - & 16.5 & 4.8 & 10.4 & 18.5 & 21.3 \\
\hline Kukenthal & 4.1 & 5.4 & 14.0 & 13.5 & 5.5 & 13.2 & 2.8 & 14.8 & - & 7.9 & 2.1 & 14.8 \\
\hline Goode & 2.3 & 21.1 & 18.6 & 15.9 & 17.2 & 17.1 & 17.8 & 25.4 & 10.5 & - & 16.9 & 16.1 \\
\hline Verrill & 3.4 & 22.2 & 4.2 & 7.3 & 4.1 & 4.8 & 20.2 & 12.5 & 13.1 & 9.9 & - & 7.8 \\
\hline Lyman & 9.2 & 13.5 & 3.6 & 13.0 & 16.7 & 15.4 & 12.2 & 4.6 & 11.5 & 15.5 & 7.2 & - \\
\hline
\end{tabular}

Table 11. Pairwise estimates of migration for the $16 \mathrm{~S}$ dataset of $O$. oedipus. The rows are the sending population and the columns are the receiving populations.

\begin{tabular}{|c|c|c|c|c|c|c|c|c|c|c|c|c|}
\hline & Bear & Balanus & Kelvin & Manning & Rehoboth & Nashville & Muir & Kukenthal & Goode & $\begin{array}{l}\text { Milne- } \\
\text { Edwards }\end{array}$ & Verrill & Lyman \\
\hline Bear & - & 24.3 & 23.7 & 22.9 & 22.6 & 23.9 & 22.1 & 24.2 & 18.7 & 23.3 & 22.9 & 20.8 \\
\hline Balanus & 23.9 & - & 23.4 & 22.3 & 22.4 & 24.3 & 21.7 & 23.9 & 18.4 & 23.4 & 23.2 & 21.0 \\
\hline Kelvin & 23.8 & 24.2 & - & 22.4 & 23.1 & 23.7 & 21.3 & 24.4 & 18.4 & 23.4 & 22.4 & 21.8 \\
\hline Manning & 22.7 & 22.2 & 22.0 & - & 20.7 & 22.4 & 20.6 & 22.0 & 18.4 & 22.4 & 21.9 & 20.8 \\
\hline Rehoboth & 22.8 & 23.1 & 22.8 & 21.8 & - & 22.8 & 20.4 & 22.8 & 18.1 & 22.8 & 22.7 & 20.5 \\
\hline Nashville & 22.6 & 23.4 & 23.4 & 21.6 & 21.9 & - & 20.6 & 23.0 & 18.5 & 22.8 & 23.1 & 20.5 \\
\hline Muir & 20.8 & 21.8 & 21.2 & 20.4 & 20.5 & 21.6 & - & 21.4 & 17.2 & 20.3 & 21.3 & 18.7 \\
\hline Kukenthal & 24.0 & 24.2 & 24.3 & 23.1 & 23.0 & 24.5 & 22.3 & - & 19.1 & 23.1 & 23.8 & 22.0 \\
\hline $\begin{array}{l}\text { Goode } \\
\text { Milne- }\end{array}$ & 17.9 & 18.0 & 17.5 & 17.1 & 17.0 & 17.6 & 16.2 & 18.2 & - & 18.0 & 17.6 & 16.2 \\
\hline Edwards & 22.6 & 23.4 & 23.1 & 22.0 & 22.9 & 23.4 & 21.0 & 23.2 & 17.9 & - & 22.4 & 20.8 \\
\hline Verrill & 22.6 & 23.3 & 23.3 & 21.4 & 22.4 & 22.5 & 20.8 & 22.9 & 18.2 & 23.2 & - & 20.4 \\
\hline Lyman & 21.1 & 22.4 & 21.5 & 19.9 & 21.4 & 21.7 & 19.1 & 21.3 & 17.3 & 21.8 & 20.5 & - \\
\hline
\end{tabular}


Table 12. Pairwise estimates of migration for the COI dataset of $O$. oedipus. The rows are the sending population and the columns are the receiving populations.

\begin{tabular}{|c|c|c|c|c|c|c|c|c|c|c|}
\hline & Balanus & Kelvin & Rehoboth & Nashville & Muir & Kukenthal & Goode & $\begin{array}{l}\text { Milne- } \\
\text { Edwards }\end{array}$ & Verrill & Lyman \\
\hline Balanus & - & 22.6 & 22.7 & 19.7 & 22.5 & 23.2 & 17.9 & 23.0 & 23.0 & 22.9 \\
\hline Kelvin & 23.2 & - & 22.8 & 19.6 & 21.4 & 23.0 & 18.9 & 23.2 & 22.9 & 21.7 \\
\hline Rehoboth & 23.0 & 22.8 & - & 19.7 & 22.2 & 22.8 & 18.8 & 23.1 & 23.1 & 23.0 \\
\hline Nashville & 19.2 & 19.0 & 19.1 & - & 18.3 & 18.4 & 16.0 & 18.9 & 18.7 & 18.9 \\
\hline Muir & 21.7 & 22.0 & 21.8 & 19.7 & - & 21.8 & 18.3 & 21.7 & 22.1 & 21.6 \\
\hline Kukenthal & 23.6 & 23.1 & 21.9 & 20.1 & 21.9 & - & 18.0 & 22.1 & 22.6 & 21.8 \\
\hline Goode & 15.8 & 15.4 & 16.0 & 14.4 & 15.1 & 15.6 & - & 15.5 & 15.2 & 15.6 \\
\hline Milne-Edwards & 24.6 & 23.7 & 23.4 & 20.4 & 22.4 & 23.9 & 18.8 & - & 24.1 & 23.8 \\
\hline Verrill & 22.9 & 23.8 & 23.3 & 20.8 & 22.3 & 23.8 & 19.1 & 23.6 & - & 23.0 \\
\hline Lyman & 22.6 & 22.9 & 22.1 & 19.3 & 21.5 & 22.5 & 18.8 & 22.2 & 22.4 & - \\
\hline
\end{tabular}

Table 13. Pairwise estimates of migration for both $16 \mathrm{~S}$ and $\mathrm{COI}$ of $O$. oedipus. The rows are the sending population and the columns are the receiving populations.

\begin{tabular}{|c|c|c|c|c|c|c|c|c|c|c|c|c|}
\hline & Bear & Balanus & Kelvin & Manning & Rehoboth & Nashville & Muir & Kukenthal & Goode & $\begin{array}{l}\text { Milne- } \\
\text { Edwards }\end{array}$ & Verrill & Lyman \\
\hline Bear & - & 18.6 & 18.2 & 19.7 & 17.6 & 8.1 & 20.1 & 18.3 & 17.0 & 19.8 & 19.3 & 18.9 \\
\hline Balanus & 14.6 & - & 14.2 & 15.2 & 13.1 & 5.4 & 14.9 & 13.6 & 13.5 & 15.3 & 14.9 & 14.3 \\
\hline Kelvin & 17.3 & 16.8 & - & 16.5 & 15.7 & 6.7 & 17.7 & 15.5 & 16.4 & 17.8 & 16.6 & 17.7 \\
\hline Manning & 20.2 & 19.3 & 19.9 & - & 18.2 & 7.8 & 21.3 & 17.8 & 18.7 & 20.7 & 20.4 & 20.3 \\
\hline Rehoboth & 13.4 & 12.5 & 12.8 & 13.3 & - & 5.1 & 13.0 & 12.4 & 11.8 & 13.4 & 13.7 & 13.1 \\
\hline Nashville & 3.8 & 3.8 & 3.9 & 3.8 & 3.8 & - & 3.9 & 3.6 & 3.8 & 3.9 & 4.0 & 3.9 \\
\hline Muir & 18.5 & 16.9 & 18.7 & 19.1 & 17.6 & 7.4 & - & 17.0 & 17.2 & 19.4 & 18.5 & 18.1 \\
\hline Kukenthal & 13.3 & 13.0 & 12.3 & 13.0 & 12.3 & 5.4 & 13.5 & - & 11.9 & 13.1 & 13.3 & 12.9 \\
\hline $\begin{array}{l}\text { Goode } \\
\text { Milne- }\end{array}$ & 9.8 & 10.0 & 9.8 & 10.0 & 9.3 & 3.6 & 9.7 & 9.2 & - & 9.5 & 9.7 & 9.8 \\
\hline Edwards & 20.3 & 20.6 & 21.0 & 22.0 & 19.5 & 8.0 & 21.0 & 19.8 & 19.9 & - & 21.0 & 21.0 \\
\hline Verrill & 18.5 & 18.7 & 18.9 & 19.4 & 18.1 & 7.6 & 18.6 & 17.4 & 17.8 & 19.4 & - & 17.7 \\
\hline Lyman & 16.8 & 15.7 & 16.1 & 16.6 & 15.2 & 6.5 & 17.2 & 14.3 & 15.3 & 16.4 & 16.2 & - \\
\hline
\end{tabular}


Table 14. Pairwise estimates of migration for the $16 \mathrm{~S}$ dataset of $O$. abyssalis. The rows are the sending population and the columns are the receiving populations.

\begin{tabular}{|l|cccccc|}
\hline & Balanus & Kelvin & Rehoboth & Nashville & Kukenthal & Goode \\
\hline Balanus & - & 14.0 & 7.2 & 13.2 & 11.8 & 11.7 \\
Kelvin & 15.7 & - & 8.5 & 18.0 & 16.5 & 15.1 \\
Rehoboth & 4.4 & 4.9 & - & 4.8 & 4.6 & 4.9 \\
Nashville & 12.4 & 17.9 & 8.2 & - & 16.9 & 17.4 \\
Kukenthal & 9.5 & 11.1 & 6.0 & 10.0 & - & 9.8 \\
Goode & 10.4 & 13.3 & 5.7 & 13.5 & 11.6 & - \\
\hline
\end{tabular}

Table 15. Pairwise estimates of migration for the $16 \mathrm{~S}$ dataset of $O$. chelys. The rows are the sending population and the columns are the receiving populations.

\begin{tabular}{|l|cccccccc|}
\hline & Picket & Balanus & Kelvin & Manning & Rehoboth & Goode & Verrill & Lyman \\
\hline Picket & - & 24.9 & 24.8 & 26.3 & 24.9 & 14.4 & 17.4 & 24.4 \\
Balanus & 24.3 & - & 25.5 & 24.3 & 25.0 & 13.5 & 16.0 & 24.5 \\
Kelvin & 22.6 & 22.3 & - & 23.0 & 23.5 & 13.5 & 15.3 & 22.5 \\
Manning & 25.7 & 25.9 & 26.3 & - & 26.2 & 14.7 & 16.7 & 26.8 \\
Rehoboth & 25.5 & 25.4 & 24.7 & 25.5 & - & 14.1 & 17.5 & 26.4 \\
Goode & 10.1 & 10.0 & 10.6 & 10.3 & 10.7 & - & 7.6 & 10.4 \\
Verrill & 15.6 & 16.0 & 16.3 & 16.0 & 16.1 & 10.3 & - & 16.1 \\
Lyman & 24.1 & 24.1 & 24.3 & 23.4 & 25.8 & 14.5 & 16.8 & - \\
\hline
\end{tabular}

Table 16. Populations receiving less migrants/generation. "=" indicates relatively equal exchange among all populations.

\begin{tabular}{|c|c|c|c|}
\hline & $16 \mathrm{~S}$ & $\mathrm{COI}$ & Both loci \\
\hline A. clavigera & Manning & $=$ & $=$ \\
\hline $\begin{array}{l}\text { O. oedipus } \\
\text { O. abyssalis } \\
\text { O. chelys }\end{array}$ & $\begin{array}{c}\text { Balanus, Nashville, Rehoboth } \\
\text { Rehoboth } \\
\text { Goode, Verrill }\end{array}$ & Balanus, Nashville, Rehoboth & Balanus, Nashville, Rehoboth \\
\hline
\end{tabular}




\section{Discussion}

This study is a multispecies and multimarker view of the extent of genetic connectivity within the North Atlantic seamounts comparing four ophiuroid species to look for congruence and differences in their patterns of connectivity and dispersal. It allows for a comparison between the three seamount regions in this study, the New England seamount chain, the Corner Rise seamounts, and Muir seamount, as well as a comparison of four ophiuroid species, three known to be associates of coral and one that is not. The main findings of this study are discussed below.

Most of the seamount peak populations within this study have undergone rapid population expansion. The haplotype networks for the $16 \mathrm{~S}$ datasets of $A$. clavigera, $O$. oedipus, and $O$. chelys all showed star-like phylogenies with an abundant root-haplotype with many closely associated rare haplotypes, which is indicative of rapid population expansion (Slatkin and Hudson 1991; Teske et al. 2005). This was further supported by the statistically significant negative Tajima $\mathrm{D}$ values for the Rehoboth populations of $A$. clavigera and $O$. chelys, and the results of the mismatch distributions which could not reject the null hypothesis of a model of expansion for all of the populations except Muir in the $16 \mathrm{~S} A$. clavigera dataset and Kukenthal in the $O$. abyssalis dataset (Table 3, Table 5, Table 7, Figure 6, and Figure 7) (Aris-Brosou and Excoffier 1996; Tajima 1996). This population expansion can explain the distribution of the haplotypes of all the molecular markers seen in all four species of ophiuroids. From the intraspecific sequence divergences for the four species of ophiuroids, this recent population expansion has been estimated to have occurred 86000-97000 years ago for A. clavigera, 29000-32000 years ago for O. oedipus, 314000-355000 years ago for O. abyssalis, and 429000-483000 years ago for $O$. chelys (Chapter 3).

There was no clear genetic break throughout the New England, Corner Rise, and Muir seamounts. Haplotypes were shared throughout the area. This can especially be seen within the $16 \mathrm{~S}$ datasets for $A$. clavigera and $O$. oedipus where one haplotype seemed to dominate the entire area indicating widespread gene flow throughout the region. This 
widespread gene flow is further supported by the result that significant structure was seen among seamount populations within the seamount regions and within seamounts in the COI dataset of $A$. clavigera and the $16 \mathrm{~S}$ dataset of $O$. chelys. The overall result among seamounts, however, showed high levels of gene flow. This implies another mechanism, such as long distance dispersal, which is swamping out the genetic structure seen within seamounts by distributing them across the region.

There were several unique haplotypes present within these datasets however and more occurred in the New England seamount chain than in the Corner Rise seamounts. Thirteen of $2216 \mathrm{~S}$ haplotypes were unique to the New England seamounts for $A$. clavigera and 1 unique to Muir seamount and for O. oedipus, three $16 \mathrm{~S}$ haplotypes were unique to the New England seamounts while one was unique to the Corner Rise region (Figure 9 and Figure 11). The distribution of 16S haplotypes of $O$. abyssalis show a similar pattern as well with haplotypes commonly distributed throughout the New England and Corner Rise seamounts and six haplotypes restricted to the New England seamounts and two restricted to the Corner Rise seamounts (Figure 7).

The haplotype distribution for O. oedipus is similar to the pattern of distribution seen in its coral host, Metallogorgia melanotrichos. Preliminary genetic analyses have shown no genetic variation for this coral throughout the entire area (Shank 2008). However, this is not so for the $16 \mathrm{~S}$ haplotype distribution of $A$. clavigera when compared to its coral host Paramuricea sp., which has been shown to have some genetic structure. Preliminary genetic analysis of the $m s h 1$ gene of Paramuricea $s p$. has shown three clades across this region, one clade distributed throughout the entire region except for the easternmost Corner Rise seamount, the second clade distributed throughout the region but absent from Muir seamount, and the third clade restricted to the westernmost New England seamounts and the continental slope (Shank 2008).

Ophiuroids are known to have a wide-range of reproductive strategies (Hendler 1991). Reproductive studies of $A$. clavigera and O. oedipus have shown them to be broadcast spawners with large yolky eggs (C. Mosher personal communication). Although no reproductive studies have determined the mode of reproduction for $O$. 
abyssalis or $O$. chelys, studies of species from the same family, Ophiacanthidae, found simple, benthic, lecithotrophic larvae characterized by either brooding, viviparity, or direct development (Hendler 1991; McEdward and Miner 2001).

This potential difference in reproductive strategy may relate to the difference in genetic differentiation we have seen in this study. Both $A$. clavigera and $O$. oedipus have the potential to be dispersed across the seamounts in the North Atlantic as they are broadcast spawners. Also, modeling studies of the circulation in this area have shown recirculation gyres resulting from the Gulf stream and its interaction with the New England seamount chain acting at depths of $3000 \mathrm{~m}$ (Figure 2) (Qiu 1994). These gyres could act as mechanisms of dispersal facilitating gene flow across the seamounts. If $O$. abyssalis or $O$. chelys had simple, benthic, lecithotrophic larvae as was shown for others in the same family, their potential for dispersal may be much lower. This may explain why populations of $O$. chelys showed significant differentiation within populations in the 16S dataset. However, this significance was slight and reproductive studies of these species would also be informative. Based on the genetic data, it may be that they share a similar mode of reproduction as $A$. clavigera and $O$. oedipus.

The slight genetic structure observed within the COI dataset for $A$. clavigera was mainly accounted for by genetic variation within a population or among populations within a region (Table 4). COI in general showed greater haplotype and nucleotide diversity than $16 \mathrm{~S}$ in $A$. clavigera (Table 3). This genetic differentiation is further seen in the haplotype network for COI haplotypes of $A$. clavigera which showed only 1 haplotype shared by two individuals and the remaining 21 haplotypes were all unique (Figure 4). This high haplotype diversity can account for the significant structure observed in the AMOVA. Further, the COI haplotype network also appeared very different from the 16S haplotype network for this species (Figure 3, Figure 4). Rather than a star phylogeny like for $16 \mathrm{~S}$ where populations are characterized by an abundant root-haplotype with many closely associated rare haplotypes, the COI haplotype network was characterized by a less abundant root haplotype and derived haplotypes that differed by several nucleotide substitutions and were not as rare in occurrence. These populations 
are thought to be older than those characterized by a star phylogeny (Slatkin and Hudson 1991; Teske et al. 2005). Variation was also seen in the haplotype networks for 16S and $\mathrm{COI}$ of $O$. oedipus, although not as clear possibly because of the low sample number (Figure 5, Figure 6).

There are several possible reasons for this contrasting result between the $16 \mathrm{~S}$ and $\mathrm{COI}$ datasets for these two species. One is the variability in mutation rate between $16 \mathrm{~S}$ and COI. Although 16S has been used in phylogeographic studies of ophiuroids in the past (Roy and Sponer 2002; Sponer et al. 2001; Sponer et al. 1998; Sponer and Roy 2002) it is thought to be a slowly-evolving mitochondrial gene and useful for both intraspecific and interspecific comparisons while COI has often been used in intraspecific studies due to nucleotide substitutions which can accumulate in third codon positions (De Francisco and Galetti 2005; Funk 1999; Halanych 2006; Hwang and Kim 1999). The genes showed a similar pattern in A. clavigera as well in the greater haplotype and nucleotide diversity seen in COI versus $16 \mathrm{~S}$ (Table 3). The greater sensitivity of the COI molecular marker may discern the more recent genetic history, not seen with the $16 \mathrm{~S}$ datasets.

The higher variation observed in COI in $A$. clavigera may also be a result of the fewer samples in the COI dataset as compared to the $16 \mathrm{~S}$ dataset. This may be an indication that we have undersampled the populations and the many COI haplotypes may not be as unique as they appear in this study. Undersampling was an issue faced in most of our datasets, especially for O. oedipus where sample sizes ranged from only 1- 5 (Table 1). This is a common limitation for studies in the deep-sea. Despite this limitation, these studies may give a preliminary understanding of the possible patterns that exist in the genetic connectivity of ophiuroid populations on the seamounts. Also, it is important to note that even within those datasets with higher sample sizes, such as the $16 \mathrm{~S}$ datasets of $O$. chelys and $A$. clavigera, unique haplotypes were still present; $80 \%$ of the $16 \mathrm{~S}$ haplotypes in $O$. chelys were unique to one seamount region as well as $53 \%$ of the 16S haplotypes of $A$. clavigera (Figure 3, Figure 8, Figure 9, Figure 14). Also, the 
COI haplotypes often vary by my more than 1 nucleotide base change which would indicate more mutations occurring within this gene as opposed to $16 \mathrm{~S}$.

Genetic isolation by distance was slightly significant for O. oedipus (Table 5). This would indicate that $O$. oedipus fits a stepping-stone model of dispersal (Kimura and Weiss 1964; Wright 1931; Wright 1951). As seamounts are often distributed in linear chains and provide discrete hard substrate habitat in the abyssal plains, it has been hypothesized that seamounts fauna may follow a stepping-stone model of dispersal (Hubbs 1959; Rogers 1994). However, this result may be due to the unique haplotype at Balanus seamount and a sampling artifact (Figure 11). More samples would help to clarify how strong this correlation is.

Similar results have been found in other studies at seamounts. Samadi et al. found no genetic structure among four crustaceans and one planktotrophic gastropod from the Norfolk seamounts in the South Pacific (Samadi et al. 2006). However, the same study did show significant differentiation and a model of isolation by distance for one nonplankotrophic gastropod. Also, slight population differentiation was found for the coral Corallium lauuense across the seamounts and islands in the Hawaiian Archipelago (Baco and Shank 2005).

Despite the high levels of gene flow, no congruent patterns of dispersal could be detected for all four species of ophiuroids. Although all populations had high levels of migration, different seamount populations experienced reduced levels of migration depending upon the species and molecular marker. This lack of congruent patterns of genetic connectivity indicates the complicated natured of connectivity and the interaction of a variety of physical and biological factors that may effect dispersal, such as reproductive strategy and associations with other taxa.

Based on the $16 \mathrm{~S}$ dataset for all four species, there is high gene flow for all four species of ophiuroids across the New England seamount chain, the Corner Rise seamounts, and Muir seamount. This was seen for both the A. clavigera and O. oedipus, ophiuroids that are broadcast spawners and have the potential for widespread distribution throughout the area by the currents within the system, as well as for $O$. abyssalis and $O$. 
chelys, whose mode of reproduction is not yet known. There was some discrepancy when comparing ophiuroids with varying degrees of relationships with host corals. $O$. chelys, who has no host, showed significant differentiation within populations, but while there may be differentiation within a population, there appears to be a long-distance dispersal mechanism like the recirculation gyres formed by the Gulf Stream and its interaction with the seamounts that act to swamp out. O. oedipus also may have a stepping-stone model of dispersal. There was also no congruent pattern of dispersal and migration for all four species.

The COI dataset for $A$. clavigera and O. oedipus showed greater hapotypic diversity than their $16 \mathrm{~S}$ datasets and slight differentiation within populations was detected for A. clavigera. This gene has greater sensitivity and may show the more recent evolutionary history than $16 \mathrm{~S}$.

Future studies are needed using a more sensitive molecular marker that may detect the more recent genetic history not observable with 16S. Also, it is important to consider a variety of taxa when trying to understand the population connectivity of a system, as many factors specific to the life history of the model system can greatly impact the result of the study. This study indicates that although there may be high gene flow within a system, different patterns of dispersal and migration can exist which may be dependent upon a complex interaction of physical factors as well as the life history characteristics of the study organism. 


\section{References}

A. B. Smith, G. L. J. P. B. L. 1995. Ophiuroid phylogeny and higher taxonomy: morphological, molecular and palaeontological perspectives. Zoological Journal of the Linnean Society (prior to Jan 1, 2002) 114: 213.

Aвoim, M. A., G. M. Menezes, T. Schlitt, and A. D. Rogers. 2005. Genetic structure and history of populations of the deep-sea fish Helicolenus dactylopterus (Delaroche, 1809) inferred from mtDNA sequence analysis. Mol Ecol 14: 13431354.

ArIS-Brosou, S., and L. ExCOFFIER. 1996. The impact of population expansion and mutation rate heterogeneity on DNA sequence polymorphism. Molecular Biology and Evolution 13: 494-504.

BACO, A. R., and T. M. SHANK. 2005. Population genetic structure of the Hawaiian precious coral Corallium lauuense (Octocorallia: Coralliidae) using microsatellites, p. 663-678. In A. Freiwald and J. M. Roberts [eds.], Cold-water Corals and Ecosystems. Springer-Verlag.

BAric, S., and C. Sturmbauer. 1999. Ecological Parallelism and Cryptic Species in the Genus Ophiothrix Derived from Mitochondrial DNA Sequences. Molecular Phylogenetics and Evolution 11: 157-162.

BATIZA, R. 2001. Seamounts and Off-Ridge Volcanism, p. 2696-2708. In J. H. Steele, S. A. Thorpe and K. K. Turekian [eds.], Encyclopedia of Ocean Sciences. Academic Press.

BeERLI, P., and J. FELSENSTEIN. 2001. Maximum-likelihood estimation of a migration matrix and effective population size in $n$ subpopulations by using a coalescent approach. Proceedings of the National Academy of Sciences 98: 4563-4568.

BOEHLERT, G. W. 1988. Current-topography interactions at mid-ocean seamounts and the impact on pelagic ecosystems. Geojournal 16: 45-52.

Boehlert, G. W., C. D. Wilson, and K. Mizuno. 1994. Populations of the sternoptychid fish Maurolicus muelleri on seamounts in the Central North Pacific. Pacific Science 48: 57-69.

Bolsch, C. J. S., N. G. Elliott, and R. D. Ward. 1993. Enzyme variation in southeastern Australian samples of the blue-eye or deepsea trevalla, Hyperoglyphe antarctica Carmichael 1818 (Teleostei: Stromateoidei). Australian Journal of Marine and Freshwater Research 44: 687-697.

Borets, L. A. 1979. The Population Structure of the Boarfish, Pentaceros richardsoni, From the Emperor Seamounts and the Hawaiian Ridge. Journal of Ichthyology 19: $15-20$.

Bruno, J. F., J. J. Stachowicz, and M. D. Bertness. 2003. Inclusion of facilitation into ecological theory, p. 119-125, Trends Ecol Evol.

Bucklin, A., R. R. Wilson, JR., and K. L. SMith, JR. 1987. Genetic differentiation of seamount and basin populations of the deep-sea amphipod Eurythenes gryllus. DEEP-SEA RES. (A OCEANOGR. RES. PAP.) 34: 1795-1810. 
CAIRNS, S. D. 2007. Studies on the western Atlantic Octocorallia (Gorgonacea:

Primnoidae). Part 8: New records of Primnoidae form the New England and Corner Rise Seamounts. Proceedings of the Biological Society of Washington 120: $243-263$.

Castelloe, J., and A. R. Templeton. 1994. Root probablities for intraspecific gene trees under neutral coalescent theory. Molecular Phylogenetics and Evolution 3: 102-113.

Clark, M. 2001. Are deepwater fisheries sustainable? -- the example of orange roughy (Hoplostethus altanticus) in New Zealand. Fisheries Research 51: 123-135.

Clement, M., D. Posada, and K. A. CRAndall. 2000. TCS: a computer program to estimate gene genealogies. Molecular Ecology 9: 1657-1660.

CRAndall, K. A., and A. R. Templeton. 1993. Empirical tests of some predicitions from coalescent theory with applications to intraspecific phylogeny reconstruction. Genetics 134: 959-969.

DE Francisco, A. K., and P. M. Galetti. 2005. Genetic distance between broodstocks of the marine shrimp Litopenaeus vannamei (Decapoda, Penaeidae) by mtDNA analyses, p. 258-261, Genet Mol Biol.

EMSON, R. H., and J. D. WoODLEY. 1987. Submersible and laboratory observations on Asteroschema tenue, a long-armed euryaline brittle star epizoic on gorgonians. Marine Biology 96: 31-45.

EpP, D., and N. C. SMOот. 1989. Distribution of seamounts in the North Atlantic. Nature 337: 254-257.

ExCOFfier, L., G. LAVAL, and S. SchNeIDER. 2005. Arlequin ver. 3.0: An integrated software package for population genetics data analysis. Evolutionary Bioinformatics Online 1: 47-50.

EzER, T. 1994. On the interaction between the Gulf Stream and the New England Seamount Chain. Journal of Physical Oceanography 24: 191-204.

Fock, H., F. Uiblen, F. Koster, and H. von Westernhagen. 2002. Biodiversity and species-environment relationships of the demersal fish assemblage at the Great Meteor Seamount (subtropical NE Atlantic), sampled by different trawls. Marine Biology 141: 185-199.

France, S. C., and T. D. KoCHER. 1996. Geographic and bathymetric patterns of mitochondrial 16S rRNA sequence divergence among deep-sea amphipods, Eurythenes gryllus. Marine Biology 126: 633-643.

FRYER, P., and G. J. FRYER. 1987. Origins of nonvolcanic seamounts in a forearc an environment. In B. Keating, P. Fryer, R. Batiza and G. W. Boehlert [eds.], Seamounts, Islands, and Atolls. Geophysical Monograph. American Geophysical Union.

FUNK, D. J. 1999. Molecular systematics of cytochrome oxidase I and 16S from Neochlamisus leaf beetles and the importance of sampling, p. 67-82, MolBiolEvol.

Grasshoff, M. 1972. The Gorgonaria of the eastern North Atlantic and the Mediterranean. I. Family Ellisellidae (Cnidaria: Anthozoa). Results of the 
'Atlantic Seamount Cruises 1967' with R. V. 'Meteor'. Meteor Forschungsergeb. 10: 73-87.

HALANYCH, K. 2006. A review of molecular markers used for Annelid phylogenetics. Integrative and Comparative Biology 46: 533-543.

Hamilton, E. L. 1956. Sunken islands of the Mid-Pacific Mountains. Geological Society of America Memoir 64.

HendLER, G. 1991. Echinodermata: Ophiuroidea, p. 355-511. In A. C. Giese, J. S. Pearse and V. B. Pearse [eds.], Reproduction of Marine Invertebrates. Boxwood Press.

HeY, J., and R. M. KLIMAN. 1993. Population genetics and phylogenetics of DNA sequence variation at multiple loci within the Drosophila melanogaster species complex. Molecular Biology and Evolution 10: 804-822.

Houghton, R. L., G. ThOMPSON, and W. B. BRYAN. 1977. Petrological and geochemical studies of the New England seamount chain. Eos, Transactions, American Geophysical Union $\mathbf{5 8 .}$

HuBBS, C. L. 1959. Initial Discoveries of Fish Faunas on Seamounts and Offshore Banks in the Eastern Pacific. Pacific Science 13: 311-316.

Hunter, R., and K. HALANYCH. 2008. Evaluating Connectivity in the Brooding Brittle Star Astrotoma agassizii across the Drake Passage in the Southern Ocean. Journal of Heredity 99: 137-148.

HWANG, U. W., and W. KIM. 1999. General properties and phylogenetic utilities of nuclear ribosomal DNA and mitochondrial DNA commonly used in molecular systematics. Korean J Parasitol 37: 215-228.

KaISER, M. J. 1998. Significance of Bottom-Fishing Disuturbance. Conservation Biology 12: $1230-1235$.

KATOH, K., K. Misawa, K. Kuma, and T. MiYata. 2002. MAFFT: a novel method for rapid multiple sequence alignment based on fast Fourier transform. Nucleic Acids Res 30: 3059-3066.

KiMURA, M. 1980. A simple method for estimating evolutionary rates of base substitutions through comparative studies of nucleotide sequences. Journal of Molecular Evolution 16: 111-120.

KimURA, M., and G. H. WeISS. 1964. The stepping-stone model of genetic structure and the decrease of genetic correlation with distance. Genetics 49: 561-576.

KLIMAN, R. M., and J. HEY. 1993. DNA sequence variation at the period locus within and among species of the Drosophila melanogaster complex Genetics 133.

Koslow, J. A. 2001. Fish stocks and benthos of seamounts. BfN Skripten 43.

Koslow, J. A., G. W. Boehlert, J. D. M. Gordon, R. L. Haedrich, P. Lorance, and N. PARIN. 2000. Continental slope and deep-sea fisheries: implications for a fragile ecosystem. ICES Journal of Marine Science 57: 548-557.

MAdDison, W. P., and D. R. MAdDISON. 2000. MacClade. Sinauer Associates.

MANTEL, N. 1967. The detectino of disease clustering and a generalized regressin approach. Cancer Research 27: 209-220.

McEdward, L. R., and B. G. Miner. 2001. Larval and life-cycle patterns in echinoderms. Canadian Journal of Zoology 79: 1125-1170. 
Moore, J. A. and others 2003. Biodiversity of Bear Seamount, New England Seamount chain: Results of exploratory trawling. Journal of Northwest Atlantic Fishery Science 31: 363-372.

NAFO. 2006. Proposal on precautionary closure to four seamount areas based on the ecosystem approach to fisheries. In N. A. F. Organization [ed.].

PAlumbi, S. R. 2003. Population genetics, demographic connectivity, and the design of marine reserves. Ecological Applications 13: 146-158.

Palumbi, S. R., A. Martin, S. Romano, W. O. McMillan, L. Stice, and G. GRABOWSKI. 1991. The simple fool's guide to PCR, 2nd Ed. Dept. Zoology, University of Hawaii, .

Parin, N. V., A. N. Mironov, and K. N. Nesis. 1997. Biology of the Nazca and Sala y Gomez Submarine Ridges, an Outpost of the Indo-West Pacific Fauna in the Eastern Pacific Ocean: Composition and Distribution of the Fauna, its Communities and History. Advances in Marine Biology 32: 145-242.

Probert, P. K. 1999. Seamounts, sanctuaries, and sustainability: moving towards deepsea conservation. Aquatic Conservation: Marine and Freshwater Ecosystems 9: 601-605.

Probert, P. K., D. G. McKnight, and S. L. Grove. 1997. Benthic invertebrate bycatch from a deep-water trawl fishery, Chatham Rise, New Zealand. Aquatic Conservation: Marine and Freshwater Ecosystems 7: 27-40.

QIU, B. 1994. Determining the mean Gulf Stream and its recirculations through combining hydrographic and altimetric data. Journal of Geophysical Research 99: 951-962.

Richards, V. P., J. D. Thomas, M. J. Stanhope, and M. S. ShivJi. 2007. Genetic connectivity in the Florida reef system: comparative phylogeography of commensal invertebrates with contrasting reproductive strategies. Mol Ecol 16: 139-157.

Richer de Forges, B., J. A. Koslow, and G. C. B. Poore. 2000. Diversity and endemism of the benthic seamount fauna in the southwest Pacific. Nature 405: 944-947.

Rogers, A. D. 1994. The biology of seamounts. Advances in Marine Biology 30: 305350.

---. 2004. The Biology, Ecology, and Vulnerability of Seamount Communities. International Union for Conservation of Nature \& Natural Resources.

Rowden, A. A., S. O'SHEA, and M. R. CLARK. 2002. Benthic biodiversity of seamounts on the northwest Chatham Rise, p. 21, Marine Biodiversity Biosecurity Report Ministry of Fisheries.

ROY, M. S., and R. SPONER. 2002. Evidence of a human-mediated invasion of the tropical western Atlantic by "the world's most common brittle star". Proceedings of the Royal Society of London. B 269: 1017-1023.

SAmadi, S., L. Bottan, E. Macpherson, B. Richer De Forges, and M. Boisselier. 2006. Seamount endemism questioned by the geographic distribution and population genetic structure of marine invertebrates. Marine Biology 149: 14631475. 
SHANK, T. M. 2008. Linkages and connectivity of cold-water coral communities along the slope, canyons, and seamounts in the North Atlantic Ocean, TRACES: TransAtlantic Coral Ecosystem Study.

Sibert, J., K. N. Holland, and D. G. ItANO. 2000. Exchange rates of yellowfin and bigeye tunas and fishery interaction between Cross seamount and near-shore FADs in Hawaii. Aquatic Living Resources 13: 225-232.

SlatKIN, M., and R. R. HudSON. 1991. Pairwise comparisons of mitochondrial DNA sequences in stable and exponentially growing populations. Genetics 129: 555562.

SPONER, R., D. DEHEYN, and M. S. Roy. 2001. Large genetic distances within a population of Amphipholis squamata (Echinodermata; Ophiuroidea) do not support colour varieties as sibling species. Marine Ecology Progress Series 219: 169-175.

Sponer, R., P. V. Mladenov, and M. S. Roy. 1998. An investigation of molecular evolution in Amphipholis squamata (Echinodermata, Ophiuroidea); project outline and preliminary results. Geological Society of New Zealand Miscellaneous Publication 97: 67-69.

SPONER, R., and M. S. ROY. 2002. Phylogeographic analysis of the brooding brittle star Amphipholis squamata (echinodermata) along the coast of New Zealand reveals high cryptic genetic variation and cryptic dispersal potential. Evolution 56: 19541967.

StоскS, K. I. 2003. SeamountsOnline, a Biogeographic Information System for Seamounts, Frontiers in Biogeography, the Inaugural Meeting of the International Biogeography Society.

TAJIMA, F. 1989. Statistical method for testing the neutral mutation hypothesis by DNA polymorphism. Genetics 125: 585-593.

--- 1996. The amount of DNA polymorphism maintained in a finite population when the neutral mutation rate varies among sites. Genetics 143: 1457-1465.

TESKE, P. R. and others 2005. Molecular evidence for long-distance colonization in an Indo-Pacific seahorse lineage. Marine Ecology Progress Series 286: 249-260.

Thrush, S. F. and others 1998. Disturbance of the Marine Benthic Habitat by Commercial Fishing: Impacts at the Scale of the Fishery. Ecological Adaptations 8: $866-879$.

Walsh, P. S., D. A. MetzGer, and R. Higuchi. 1991. Chelex 100 as a medium for simple extraction of DNA and PCR-based typing from forensic material. BioTechniques 10: 506-513.

WiLSON, R. R., JR., and R. S. KaUfMANN. 1987. Seamount biota and biogeography, p. 355-377. In B. H. Keating, P. Fryer, R. Batiza and G. W. Boehlert [eds.], Seamounts, Islands, and Atolls. Geophysical Monograph Series. American Geophysical Union.

Wright, S. 1931. Evolution in Mendelian Populations. Genetics 16: 97-159.

---. 1951. The genetical structure of populations. Ann. Eugen. 15: 323-354.

ZHENG, Y., and J. ARKANI-HAMED. 2002. Rigidity of the Atlantic oceanic lithosphere beneath New England seamounts. Tectonophysics 359: 359-369. 


\section{Chapter 5: Conclusions}

The goal of my dissertation was to further our understanding of the role seamounts play in marine biodiversity and biogeography by characterizing the fauna and faunal assemblages found on seamounts, quantifying specific host-associate relationships, and identifying patterns of faunal distribution on the seamounts and the factors important for creating, structuring, and maintaining these patterns. I then focused on a particular faunal group, specifically ophiuroids, to see whether these broad community patterns were discernible on a genetic level. I assessed the usefulness of the 16S mtDNA gene as a genetic "barcode" to identify ophiuroid species in order to assess the diversity of ophiuroids on seamounts and to confirm preliminary morphospecies identifications of four target species for a population genetic study. A multispecies and multigenic population genetic study of four ophiuroid species with potentially varying life histories found on the North Atlantic seamounts was employed to assess the gene flow across the North Atlantic region.

\section{Chapter 2: Fauna-habitat associations on the New England and Corner Rise Seamounts}

This study characterized the faunal assemblages found on seamounts, identified any pattern of relatedness between those assemblages and identified the factors that are important for structuring those patterns. Videographic analysis of seamount populations found that the most abundant phyla observed were the Porifera (sponges), Cnidaria (mainly corals), and the Echinodermata (ophiuroids, sea urchin, and holothurians). The fauna were dominated by suspension feeders, which is consistent with previous studies of seamount fauna (Metaxas and Davis 2005; Rogers 1994; Stocks 2004). The benthic biomass is often dominated by large suspension feeders that provide important habitat for 
smaller, mobile invertebrates (Samadi et al. 2007). The dominance of filter feeders is thought to be related with the interaction of seamounts with the surrounding currents. Currents can help expose hard substrate to provide habitat for sessile filter feeders such as corals and sponges by removing sediment, which otherwise dominates the deep sea benthic habitat (Gage and Tyler 1991; Stocks 2004).

This study found distinct seamount assemblages structured by region, depth, and substrate. The communities in the New England Seamount chain were distinct from those in the Corner Rise seamounts. In addition to the MDS analyses, 60 morphospecies were found to be unique to the New England seamounts while 75 morphospecies were unique to the Corner Rise seamount. This result is similar to the pattern of regional differences also observed between two ridge systems in southwest Pacific seamounts, and also in regional differences found in megafauna assemblages associated with deep-water gorgonian corals in different sites in the Northeast Channel in Canada (Metaxas and Davis 2005; Richer De Forges et al. 2000). These regional differences may be caused by a variety of factors including the structure and habitat availability on seamounts, the different geological histories of the seamount groups, and the hydrographic regime of the region as modeling studies of current flow around the North Atlantic seamounts showed the formation of eddies that could isolate the two seamount groups (Qiu 1994).

There were also four distinct assemblages separated by depth, with breaks at 1300 $\mathrm{m}, 2300 \mathrm{~m}$, and $2600 \mathrm{~m}$. Similar distinct faunal assemblages based on depth have also been observed on Patton seamount in the Gulf of Alaska (Hoff and Stevens 2005). Depth has also been found to be an important factor in structuring seamount communities (Samadi et al. 2007). Similar faunal zonation by depth has been seen in other studies, particularly at the $1300 \mathrm{~m}$ and $2300 \mathrm{~m}$ depth breaks. Important faunal boundaries are thought to occur globally around 1000-1400 m depth (Howell et al. 2002). This boundary has been attributed to slope gradient, which is related to the formation of enhanced bottom currents through internal tides and can effect sediment transport and substratum type (Howell et al. 2002). The $2600 \mathrm{~m}$ break coincides with a boundary of a 
well-defined intermediate region of 2500-3500 m where bathyal fauna overlap with abyssal fauna (Haedrich et al. 1980; Howell et al. 2002).

The $2300 \mathrm{~m}$ depth break is less supported although a similar break was observed between $1947 \mathrm{~m}-2116 \mathrm{~m}$ in a study of the megabenthic fauna in the deep-sea south of New England, USA (Haedrich et al. 1980), and coincides with a peak in diversity for invertebrate megafauna in the northwest Atlantic between 1900-2300 m (Howell et al. 2002; Rex 1981). Also, SIMPER analysis revealed that the difference between the middle and deep depth regions were accounted for by disappearance of several taxa, including the disappearance of ophiuroids and coral hosts found to be tightly associated with each other. However there were only two dives in the $2300-2600 \mathrm{~m}$ range and this result may be a sampling artifact. The depth zonation patterns observed in this study are probably the result of a complex combination of physical factors and biological interactions (Gage and Tyler 1991).

However, although depth is an important structuring factor for these communities, they were more similar based on seamount than depth, indicating that geography may be a more significant factor than depth.

This study also quantified the relationships between host and associate fauna. Significant differences were observed for faunal assemblages found on different types of substrate, including natural abiotic substrate (soft sediment, hard substrate, and open water) which were seen to be distinct from each other as well as from anthropogenic abiotic substrates and biotic substrates.

Seven taxa were identified to occur at least $50 \%$ or more on the same host and observed at least 20 times or more. These significant relationships varied in degrees from $60 \%$ occurrence to $100 \%$ occurrence, indicating different levels of specificity. Hydroid sp. 2 was observed on a variety of hosts, but most often on dead parts of the host and therefore is probably not an obligate relationship. The ophiuroid Ophioplinthaca abyssalis was also observed on a variety of other hosts, but it was predominantly observed on one coral host, Candidella imbricata. Although not an obligate symbiont, it appears to have a strong preference for this host. An octopus egg case was also observed 
on a variety of hosts, but only corals. The shrimp Bathypalaemonella serratipalma was observed only on the corals Chrysogorgia spp. and Iridogorgia spp., the galatheid Uroptychus sp. 2 was observed only on the antipatharian Parantipathes sp., the ophiuroid Asteroschema clavigera was observed on the Paragorgia spp. and Paramuricea spp., and the ophiuroid Ophiocreas oedipus was observed only on the coral Metallogorgia melanotrichos.

These results indicate the unique faunal assemblages found at seamounts and that there are distinct communities structured by geographic region, depth, and substrate or host composition. As seamount communities are threatened by increased pressure from deep-sea fisheries, it is imperative to consider these factors for future conservation efforts.

\section{Chapter 3: Identifying ophiuroids from the North Atlantic seamounts using DNA barcodes}

This study provides support that a 16S-based identification system will be effective for identifying ophiuroid species. There is a much lower level of intraspecific sequence variation than interspecific sequence variation. A total of 22 putative seamount species were found. Most of the ophiuroids collected from the North Atlantic seamounts belong to the family Ophiacanthidae, which is not surprising as this family occurs primarily in deep water and many of the genera of this family are only found below the shelf edge (Tyler 1980).

The mitochondrial gene $16 \mathrm{~S}$ appears to be a viable marker for confirming species identity. It identified species and families with moderate success. The neighbour-joining analysis allowed species to cluster together, however it was not enough to delineate the phylogenetic relationships of the organisms. The resulting $16 \mathrm{~S}$ neighbour-joining tree, although clustering species into families with some success, it could not characterize the relationships between those clades. Depending upon the molecular marker used, DNA 
barcodes may be adequate to delineate species, but they may not be sensitive enough to describe phylogenetic relationships correctly between species (Desalle et al. 2005). In this case, the neighbour-joining analysis did not reflect the relationships thought to exist within the class Ophiuroidea.

In addition, although a strict molecular clock could not be applied to the phylogenetic analysis, applying an approximate echinoderm mtDNA divergence rate of $3.1-3.5 \% /$ my revealed that the ophiuroids diverged after the formation of the seamounts, within the last 10.6 million years.

Although our preliminary identifications of the ophiuroids were very poor, the four target species Asteroschema clavigera, Ophiocreas oedipus, Ophioplionthaca abyssalis, and Ophioplinthaca chelys were identified. This result emphasizes the importance of confirming the species identity of any organism that may be used for a later analysis, especially for population genetics. This was a necessary step before intraspecific analyses could be done. Also, although unable to resolve all the relationships among the seamount ophiuroids, this study found a diverse assemblage of ophiuroids that may have radiated on the seamounts.

\section{Chapter 4: Patterns of gene flow of four species of ophiuroids from North Atlantic seamounts}

This is a multispecies and multimarker study of the gene glow of four species of ophiuroids within the North Atlantic seamounts, comparing three different seamount regions in this study, the New England seamount chain, the Corner Rise seamounts, and Muir seamount, as well as a four ophiuroid species, three known to be associates of coral and one that is not.

Most of the seamount peak populations within this study appear to have undergone rapid population expansion as seen in the haplotype networks for the $16 \mathrm{~S}$ datasets of A. clavigera, O. oedipus, and $O$. chelys, the statistically significant negative 
Tajima D values for the Rehoboth populations of $A$. clavigera and $O$. chelys, and the results of the mismatch distributions which could not reject the null hypothesis of a model of expansion for all of the populations except Muir in the $16 \mathrm{~S}$ A. clavigera dataset and Kukenthal in the $O$. abyssalis dataset. This population expansion can explain the distribution of the haplotypes of all the molecular markers seen in all four species of ophiuroids. There was no clear genetic break throughout the New England, Corner Rise, and Muir seamounts. Haplotypes were shared throughout the area. This can especially be seen within the $16 \mathrm{~S}$ datasets for A. clavigera and O. oedipus where one haplotype seemed to dominate the entire area indicating widespread gene flow throughout the region. Using the intraspecific sequence divergences found in Chapter 3 and an approximate echindoerm mtDNA sequence divergence rate of 3.1-3.5\%/my, these radiations could have occurred approximately within the last 86000-97000 years for $A$. clavigera, 29000-32000 years for O. oedipus, 314000-355000 years for O. abyssalis, and 429000-483000 years for $O$. chelys.

Based on the 16S dataset for all four species, there are congruent patterns of high gene flow for all four species of ophiuroids across the New England seamount chain, the Corner Rise seamounts, and Muir seamount. This was seen for both the A. clavigera and O. oedipus, ophiuroids that are broadcast spawners and have the potential for widespread distribution throughout the area by the currents within the system, as well as for $O$. abyssalis and $O$. chelys, whose mode of reproduction is not yet known. There was some discrepancy when comparing ophiuroids with varying degrees of relationships with host corals. O. chelys, who has no host, showed significant differentiation within populations, but while there may be differentiation within a population, there appears to be a longdistance dispersal mechanism like the recirculation gyres formed by the Gulf Stream and its interaction with the seamounts that act to swamp out. O. oedipus also may have a stepping-stone model of dispersal.

All four species showed high levels of migration, approximately 20 migrants per generation for A. clavigera, O. oedipus, and $O$. chelys, but $O$. abyssalis had less, only about 10 migrants per generation. Also, different seamount populations experienced less 
levels of migration depending upon species and marker. No congruent patterns of dispersal could be found. So, although there is high gene flow in the region, each species is exhibiting a different pattern of dispersal indicating the complexity of the interaction of physical and biological factors involved in population connectivity. These differences may involve the different life histories of the ophiuroids as all four have varying levels of specificity with a coral host and potentially different modes of reproduction.

The COI dataset for A. clavigera and O. oedipus showed greater haplotypic diversity than their $16 \mathrm{~S}$ datasets and slight differentiation within populations was detected for $A$. clavigera. This gene has greater sensitivity and may show more recent evolutionary history than $16 \mathrm{~S}$.

Future studies are needed which can look for a more sensitive molecular marker that may detect the more recent genetic history, not observable with 16S. Also, studies of other taxa are needed to further clarify the different patterns of genetic connectivity shown by the four different ophiuroid species in this study.

\section{Broader impacts and future directions}

The interacting biological, physical, and hydrological characteristics of seamounts and seamount communities indicate that they are important factors in marine biodiversity, biogeography, and evolution. Seamounts may be significant links between different biogeographic provinces within the oceans acting as "stepping-stones" and playing an important part in the trans-oceanic dispersal of organisms as key faunal passages for dispersal (Hamilton 1956; Hubbs 1959; Rogers 1994). With the increasing pressures of deep-sea fisheries destroying a potentially vital link within ocean systems it is all the more important to assess the population dynamics of seamount fauna. This will not only provide a greater understanding of ocean biogeography and deep-sea biodiversity but also be an important step in guiding conservation efforts to protect the vulnerable seamount fauna. With the collapse of traditional continental shelf and pelagic fisheries and the improvement of fishing technology, deep-sea fisheries have grown and have contributed 
800,000 to 100,000 tons annually to global fish landings since 1964 (Clark 2001; Koslow et al. 2000). Deep-sea fishing techniques such as trawling and long-line fishing have been shown to destroy benthic habitat, in particular the coral habitat upon which many invertebrates live (Kaiser 1998; Probert 1999; Probert et al. 1997; Thrush et al. 1998). With expanding deep-sea fisheries and the concurrent destruction of seamount habitat, particularly of the live and fossil corals, conservation efforts for these areas have increased, like the proposal for the precautionary closure of four seamount areas in the North Atlantic to deep-sea fisheries, which include portions of the Corner and New England seamounts (NAFO 2006). Understanding the extent of genetic connectivity between seamounts and seamount areas will aid in the creation of marine protected areas and future conservation and policy efforts. Such a strategy is advocated for the conservation of coral reef communities (Palumbi 2003; Richards et al. 2007).

This study has found broad community level factors of geographic region, depth, and habitat/substrate that influence the structure of seamount communities. It is important to remember these factors when planning to protect these resources. Specifically within the North Atlantic, conservation efforts should aim to protect both the Corner Rise and New England seamount chain as separate regions and also consider the different faunal assemblages found in the depth ranges between the depth breaks of 1300m, $2300 \mathrm{~m}$, and $2600 \mathrm{~m}$. In protecting seamount communities it is important to remember that although sponges and corals may be the dominant megafauna, there is a rich and diverse community of associated fauna that depend in varying degrees to the habitat these megafauna provide. In addition, this study has identified species level factors of the life history characteristics of the fauna, reproduction strategy, and host-associate relationships, which all may influence the patterns of genetic connectivity on a species level scale. Lack of congruent patterns of genetic connectivity in these four ophiuroid species indicates the complex nature of connectivity and the interaction of a variety of physical and biological factors. This study has also shown the need for studies of a variety of taxa, especially for an ecosystem approach to management. Although broad 
community level patterns may be apparent, individual taxa may exhibit highly variable patterns of connectivity.

Future studies are needed to compare the results of this study with other seamount groups in order to ascertain whether the same factors are found to be important in different areas. Also, future studies of ophiuroids may help to further explain the results of this study, in particular comparing the reproduction strategies of the ophioplinthacid ophiuroids with $A$. clavigera and $O$. oedipus. Also, this study has shown the importance of choosing a sensitive enough marker to detect the more recent genetic history of an organism. Further studies with COI and with greater sample sizes would be justified as well as studies of additional taxa in order to get a comprehensive view of the dynamics of population and genetic connectivity within a seamount system. 


\section{REFERENCES}

ArIS-Brosou, S., and L. EXCOFFIER. 1996. The impact of population expansion and mutation rate heterogeneity on DNA sequence polymorphism. Molecular Biology and Evolution 13: 494-504.

BACO, A. R., and T. M. SHANK. 2005. Population genetic structure of the Hawaiian precious coral Corallium lauuense (Octocorallia: Coralliidae) using microsatellites, p. 663-678. In A. Freiwald and J. M. Roberts [eds.], Cold-water Corals and Ecosystems. Springer-Verlag.

BARRETT, R., and P. HEBERT. 2005. Identifying spiders through DNA barcodes. Canadian Journal of Zoology 83: 481-491.

Buhl-Mortensen, L., and P. B. Mortensen. 2004a. Crustaceans associated with the deep-water gorgonian corals Paragorgia arborea (L., 1758) and Primnoa resedaeformis (Gunn., 1763) Journal of Natural History 38: 1233-1247.

---. 2004b. Symbiosis in deep-water corals. Symbiosis 37: 33-61.

---. 2005. Distribution and diversity of species associated with deep-sea gorgonian corals off Atlantic Canada, p. 849-879. In A. Freiwald and J. M. Roberts [eds.], Coldwater Corals and Ecosystems. Springer-Verlag.

CAIRns, S. D. 2007. Studies on the western Atlantic Octocorallia (Gorgonacea: Primnoidae). Part 8: New records of Primnoidae form the New England and Corner Rise Seamounts. Proceedings of the Biological Society of Washington 120: 243-263.

Clark, M. 2001. Are deepwater fisheries sustainable? -- the example of orange roughy (Hoplostethus altanticus) in New Zealand. Fisheries Research 51: 123-135.

DE FrancisCO, A. K., and P. M. GALETTI. 2005. Genetic distance between broodstocks of the marine shrimp Litopenaeus vannamei (Decapoda, Penaeidae) by mtDNA analyses, p. 258-261, Genet Mol Biol.

DeSalle, R., M. Egan, and M. SidDAll. 2005. The unholy trinity: taxonomy, species delimitation and DNA barcoding. Philosophical Transactions of the Royal Society B: Biological Sciences 360: 1905-1916.

DuncAn, R. A. 1984. Age progressive volcanism in the New England seamounts and the opening of the central Atlantic ocean. Journal of Geophysical Research 89: 99809990.

Duncan, R. A., and D. A. Clague. 1985. Pacific Plate motion recorded by linear volcanic chains. Plenum Press, New York, NY, United States (USA).

EzER, T. 1994. On the interaction between the Gulf Stream and the New England Seamount Chain. Journal of Physical Oceanography 24: 191-204.

FUNK, D. J. 1999. Molecular systematics of cytochrome oxidase I and 16S from Neochlamisus leaf beetles and the importance of sampling, p. 67-82, MolBiolEvol. 
GAge, J. D., and P. A. TYLER. 1991. Deep-sea biology: a natural history of organisms at the deep-sea floor. Cambridge University Press, Cambridge, New York etc: 1504.

Haedrich, R. L., G. T. Rowe, and P. T. Polloni. 1980. The megabenthic fauna in the deep sea south of New England, USA. Marine Biology 57: 165-179.

HALANYCH, K. 2006. A review of molecular markers used for Annelid phylogenetics. Integrative and Comparative Biology 46: 533-543.

Hamilton, E. L. 1956. Sunken islands of the Mid-Pacific Mountains. Geological Society of America Memoir 64.

Hebert, P., A. Cywinska, S. Ball, and J. DewaArd. 2003. Biological identifications through DNA barcodes. Proceedings of the Royal Society B: Biological Sciences 270: 313-321.

Hendler, G. 1991. Echinodermata: Ophiuroidea, p. 355-511. In A. C. Giese, J. S. Pearse and V. B. Pearse [eds.], Reproduction of Marine Invertebrates. Boxwood Press.

Hoff, G. R., and B. Stevens. 2005. Faunal Assemblage Structure on the Patton Seamount (Gulf of Alaska, USA). Alaska Fishery Research Bulletin 11: 27-36.

Howell, K. L., D. S. M. Billett, and P. A. Tyler. 2002. Depth-related distribution and abundance of seastars (Echinodermata : Asteroidea) in the Porcupine Seabight and Porcupine Abyssal Plain, NE Atlantic. Deep-Sea Research I 49: 1901-1920.

HubBS, C. L. 1959. Initial Discoveries of Fish Faunas on Seamounts and Offshore Banks in the Eastern Pacific. Pacific Science 13: 311-316.

HWANG, U. W., and W. KIM. 1999. General properties and phylogenetic utilities of nuclear ribosomal DNA and mitochondrial DNA commonly used in molecular systematics. Korean J Parasitol 37: 215-228.

KAISER, M. J. 1998. Significance of Bottom-Fishing Disuturbance. Conservation Biology 12: $1230-1235$.

KIMURA, M., and G. H. WeISS. 1964. The stepping-stone model of genetic structure and the decrease of genetic correlation with distance. Genetics 49: 561-576.

Koslow, J. A., G. W. Boehlert, J. D. M. Gordon, R. L. Haedrich, P. Lorance, and N. PARIN. 2000. Continental slope and deep-sea fisheries: implications for a fragile ecosystem. ICES Journal of Marine Science 57: 548-557.

Levin, L. A., D. J. DeMaster, L. D. McCann, and C. L. Thomas. 1986. Effects of giant protozoans (class: Xenophyophorea) on deep-seamount benthos. Marine Ecology Progress Series 29: 99-104.

LEVIN, L. A., and C. L. ThOMAS. 1988. The ecology of xenophyophores (Protista) on eastern Pacific seamounts. Deep-Sea Research 12.

---. 1989. The influence of hydrodynamic regime on infaunal assemblages inhabiting carbonate sediments on central Pacific Seamounts. Deep-Sea Research 36: $1897-$ 1915.

McEDwARD, L. R., and B. G. Miner. 2001. Larval and life-cycle patterns in echinoderms. Canadian Journal of Zoology 79: 1125-1170.

MetaXAs, A., and J. DAVIS. 2005. Megafauna associated with assemblages of deepwater gorgonian corals in Northeast Channel, off Nova Scotia, Canada. Journal of the Marine Biological Association of the United Kingdom 85: 1381-1390. 
Moore, J. A. and others 2003. Biodiversity of Bear Seamount, New England Seamount chain: Results of exploratory trawling. Journal of Northwest Atlantic Fishery Science 31: 363-372.

NAFO. 2006. Proposal on precautionary closure to four seamount areas based on the ecosystem approach to fisheries. In N. A. F. Organization [ed.].

PAlumbi, S. R. 2003. Population genetics, demographic connectivity, and the design of marine reserves. Ecological Applications 13: 146-158.

Probert, P. K. 1999. Seamounts, sanctuaries, and sustainability: moving towards deepsea conservation. Aquatic Conservation: Marine and Freshwater Ecosystems 9: 601-605.

Probert, P. K., D. G. McKnight, and S. L. Grove. 1997. Benthic invertebrate bycatch from a deep-water trawl fishery, Chatham Rise, New Zealand. Aquatic Conservation: Marine and Freshwater Ecosystems 7: 27-40.

QIU, B. 1994. Determining the mean Gulf Stream and its recirculations through combining hydrographic and altimetric data. Journal of Geophysical Research 99: 951-962.

ReX, M. A. 1981. Community Structure in the Deep-Sea Benthos. Annual Review of Ecology and Systematics: 331-353.

Richards, V. P., J. D. Thomas, M. J. Stanhope, and M. S. ShivJi. 2007. Genetic connectivity in the Florida reef system: comparative phylogeography of commensal invertebrates with contrasting reproductive strategies. Mol Ecol 16: 139-157.

Richer de Forges, B., J. A. Koslow, and G. C. B. Poore. 2000. Diversity and endemism of the benthic seamount fauna in the southwest Pacific. Nature 405: 944-947.

Rogers, A. D. 1994. The biology of seamounts. Advances in Marine Biology 30: 305350.

---. 2008. Molecular ecology and phylogenetics of deep-sea species in the North Atlantic, European TRACES workshop.

ROY, M. S., and R. SPONER. 2002. Evidence of a human-mediated invasion of the tropical western Atlantic by "the world's most common brittle star". Proceedings of the Royal Society of London. B 269: 1017-1023.

SAmadi, S., L. Bottan, E. Macpherson, B. Richer De Forges, and M. Boisselier. 2006. Seamount endemism questioned by the geographic distribution and population genetic structure of marine invertebrates. Marine Biology 149: 14631475.

Samadi, S., T. Schlacher, and B. R. DE Forges. 2007. Seamount benthos, p. 119-140. In T. J. Pitcher et al. [eds.], Seamounts: Ecology, Fisheries, \& Conservation. Fish and Aquatic Resources Series. Blackwell Publishing.

SlatKIn, M., and R. R. HUDSON. 1991. Pairwise comparisons of mitochondrial DNA sequences in stable and exponentially growing populations. Genetics 129: 555562.

SLEEP, N. H. 1990. Monteregian hotspot track: a long-lived mantle plume. Journal of Geophysical Research 95: 21,983-921,990. 
Smith, A. B., G. J. L. Paterson, and B. Lafay. 1995. Ophiuroid phylogeny and higher taxonomy: morphological, molecular and palaeontological perspectives.

Zoological Journal of the Linnean Society (prior to Jan 1, 2002) 114: 213.

SPONER, R., D. DEHEYN, and M. S. Roy. 2001. Large genetic distances within a population of Amphipholis squamata (Echinodermata; Ophiuroidea) do not support colour varieties as sibling species. Marine Ecology Progress Series 219: 169-175.

SPONER, R., P. V. MLADENOV, and M. S. RoY. 1998. An investigation of molecular evolution in Amphipholis squamata (Echinodermata, Ophiuroidea); project outline and preliminary results. Geological Society of New Zealand Miscellaneous Publication 97: 67-69.

SPONER, R., and M. S. ROY. 2002. Phylogeographic analysis of the brooding brittle star Amphipholis squamata (echinodermata) along the coast of New Zealand reveals high cryptic genetic variation and cryptic dispersal potential. Evolution 56: 19541967.

STOCKS, K. I. 2004. Seamount invertebrates: composition and vulnerability to fishing, p. 17-24. In T. Morato and D. Pauly [eds.], Seamounts: Biodiversity and Fisheries. Fisheries Centre, University of British Columbia.

TAJIMA, F. 1996. The amount of DNA polymorphism maintained in a finite population when the neutral mutation rate varies among sites. Genetics 143: 1457-1465.

TESKE, P. R. and others 2005. Molecular evidence for long-distance colonization in an Indo-Pacific seahorse lineage. Marine Ecology Progress Series 286: 249-260.

Thrush, S. F. and others 1998. Disturbance of the Marine Benthic Habitat by Commercial Fishing: Impacts at the Scale of the Fishery. Ecological Adaptations 8: $866-879$.

Tyler, P. A. 1980. Deep-Sea Ophiuroids. Oceanography and Marine Biology: An Annual Review 18: 125-153.

Ward, R., T. ZemlaK, B. InNES, P. LASt, and P. HEBert. 2005. DNA barcoding Australia's fish species. Philosophical Transactions of the Royal Society B: Biological Sciences 360: 1847-1857.

Wright, S. 1931. Evolution in Mendelian Populations. Genetics 16: 97-159.

---. 1951. The genetical structure of populations. Ann. Eugen. 15: 323-354.

ZHENG, Y., and J. ARKANI-HAMED. 2002. Rigidity of the Atlantic oceanic lithosphere beneath New England seamounts. Tectonophysics 359: 359-369. 